\title{
Evolución de enanas blancas de masas bajas e intermedias
}

\author{
Tesis doctoral de Aldo M. Serenelli \\ Facultad de Ciencias Astronómicas y Geofísicas \\ Universidad Nacional de La Plata \\ Consejo Nacional de Investigaciones Científicas y \\ Tecnológicas - Instituto Astrofísico La Plata
}

Director: Dr. Leandro G. Althaus

Co-director: Dr. Omar G. Benvenuto

La Plata, 27 de Diciembre de 2002 


\section{Agradecimientos}

El trabajo realizado en estos cuatro años no hubiera sido posible sin la presencia y guía permanente Leandro Althaus. A él va mi más profundo agradecimiento por haber compartido estos años de trabajo a lo largo de los cuales también se ha desarrollado una hermosa amistad.

Omar Benvenuto ha sabido orientar mi trabajo desde mis últimos años como estudiante. Esta tesis tampoco hubiera sido posible sin su presencia. También quiero hacer extensivo mi agradecimiento a Alejandro Córsico y Jorge Panei, con quienes he compartido muchas tardes placenteras durante estos años.

Me gustaría agradecer particularmente a Rubén Martínez por el soporte técnico brindado y también a Mariano Méndez, Nidia Morrell y Juan Carlos Forte quienes en diversos momentos han ayudado a abrir algunas puertas. 


\section{Contenidos}

$\begin{array}{ll}\text { Agradecimientos } & 1\end{array}$

1 Introducción 1

1.1 Breve reseña histórica . . . . . . . . . . . . . . . . . . 1

1.2 Física general de las enanas blancas . . . . . . . . . . . . . . . . . . . . . 2

1.3 Motivación y objetivos . . . . . . . . . . . . . . . . . . 5

2 El código de evolución estelar $\quad 8$

2.1 Ecuaciones de estructura y evolución . . . . . . . . . . . . . 8

2.2 Tratamiento numérico de las ecuaciones de estructura . . . . . . . . . . 10

2.3 Ecuaciones de cambios de composición química . . . . . . . . . . . . . 12

2.4 Tratamiento numérico de las ecuaciones de cambio de composición química . . . 13

2.5 Física constitutiva . . . . . . . . . . . . . . . 16

3 Enanas blancas de baja masa I. Progenitores con metalicidad solar 18

3.1 Introducción . . . . . . . . . . . . . . . . 18

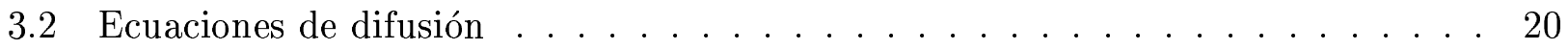

3.3 Modelos iniciales . . . . . . . . . . . . . . . . . 22

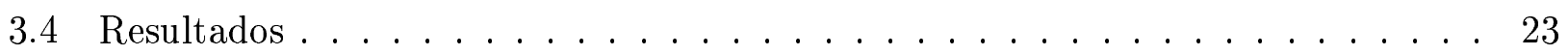

3.4.1 Comparación entre modelos con y sin difusión . . . . . . . . . . . 23

3.4 .2 Evolución durante un flash termonuclear . . . . . . . . . . . . 38

3.5 Discusión y conclusiones . . . . . . . . . . . . . . . . . 42

4 Enanas blancas de baja masa II. Índices de color $\quad 44$

4.1 Introducción . . . . . . . . . . . . . . . . . . . 44

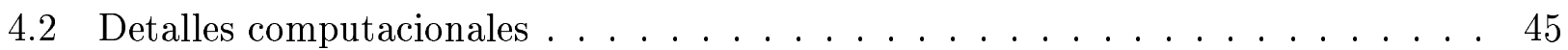

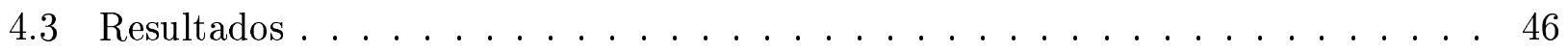

4.4 Conclusiones . . . . . . . . . . . . . . . . . 56

5 Enanas blancas de baja masa. Progenitores de baja metalicidad $\quad \mathbf{5 7}$

5.1 Introducción . . . . . . . . . . . . . . . . . 57

5.2 Modelos de atmósfera . . . . . . . . . . . . . . . . . . . . . . . . . . . . . . . . . . . . . . . 58

5.3 Modelos iniciales . . . . . . . . . . . . . . . . . 59

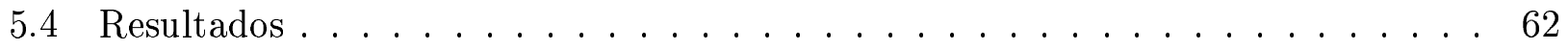

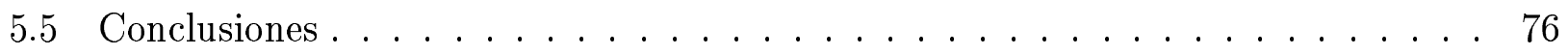


6 Enanas blancas de carbono-oxígeno. Progenitor de $3 \mathbf{M}_{\odot} \quad 79$

6.1 Introducción . . . . . . . . . . . . . . . . 79

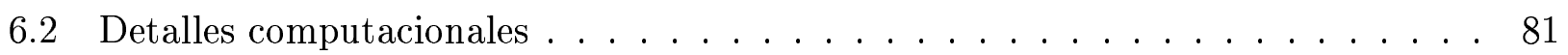

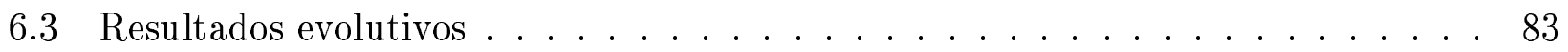

6.3 .1 Progenitor de enana blanca . . . . . . . . . . . 83

6.3.2 Evolución post-AGB y enana blanca . . . . . . . . . . . . 87

6.4 Cantidades para el análisis de las pulsaciones

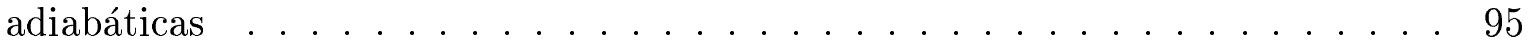

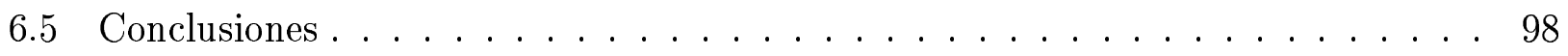

7 Enanas blancas de carbono y oxígeno. Progenitor de $7.5 \mathrm{M}_{\odot} \quad 101$

7.1 Introducción . . . . . . . . . . . . . . . . . . 101

7.2 Detalles computacionales . . . . . . . . . . . . . . 101

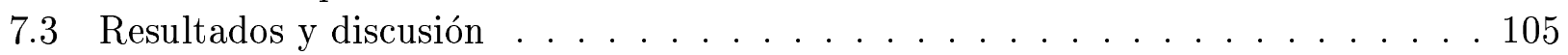

7.4 Conclusiones. . . . . . . . . . . . . . . . . 112

$\begin{array}{ll}\text { Bibliografía } & 121\end{array}$ 


\section{Capítulo 1}

\section{Introducción}

\subsection{Breve reseña histórica}

La estructura estelar ha sido objeto de una gran cantidad de estudios desde los trabajos pioneros de Lane, Ritter, Kelvin, Emden y Fowler, en las últimas décadas del siglo XIX y primeras del $\mathrm{XX}$. Estos trabajos iniciales sobre la estructura de las estrellas establecieron claramente que la mera consideración de las estrellas como esferas gaseosas mantenidas en equilibrio hidrostático gracias a su autogravitación no era suficiente para determinar su estructura. En efecto, una presión dada, requerida por la estrella para mantener el peso de sus capas superiores puede obtenerse mediante una innumerable cantidad de combinaciones de temperatura y densidad. Era claro que más condiciones eran necesarias para determinar la estructura estelar. Estas condiciones son la producción y transporte de energía térmica. En 1929, R. Atkinson y F. Houtermans fueron los primeros en mostrar que las reacciones termonucleares proveen la fuente de energía de las estrellas, corolario de las investigaciones de G. Gamow sobre el efecto tunel. En 1938, H. Bethe y Ch. Critchfield describieron por primera vez la cadena de reacciones nucleares, hoy conocidas con el nombre de cadena protón-protón, destacando el rol de las interacciones débiles en la misma. Las bases de la teoría de la estructura y evolución estelar habían sido establecidas.

Desde entonces, gran cantidad de investigaciones se han centrado en el estudio de la estructura y evolución estelar. Una enumeración aquí, aún sólo de las más relevantes, sería demasiado extensa dada la amplitud y diversidad de temas relacionados. Esta tesis está centrada principalmente en un estudio de las estrellas enanas blancas (WDs) y por este motivo, nos limitaremos a mencionar brevemente algunos descubrimientos importantes en relación con estos objetos.

En 1914, Adams (1915) descubrió que el espectro de Sirio B correspondía al de una estrella "blanca". Asignando una temperatura de $8000 \mathrm{~K}$ a la misma, y conocida su luminosidad, estimó su radio en $18800 \mathrm{~km}$ (unas cuatro veces mayor que el radio aceptado actualmente). Posteriormente, Adams (1925) determinó el corrimiento al rojo gravitatorio de varias líneas espectrales emitidas desde la superficie de Sirio B y utilizando la teoría de relatividad general pudo determinar su cociente $M / R$. Dado que la masa de Sirio B era conocida gracias al estudio de su órbita, su radio pudo ser determinado. De esta manera se pudo establecer la naturaleza "compacta" de dicho objeto, que dada su temperatura recibió el nombre genérico de enana blanca. En 1926, Fowler (1926) aplicó la entonces recientemente formulada estadística de Fermi-Dirac para explicar la naturaleza de las enanas blancas: identificó la presión que sostiene la estructura de estos objetos con la presión de degeneración de los electrones. En 1930, Chandrasekhar (1931a,b) construyó los primeros modelos realistas de enanas blancas, 
incluyendo efectos relativistas en la ecuación de estado. En el curso de estos trabajos descubrió que existe una masa máxima que podía ser soportada por presión de degeneración electrónica, límite conocido como "masa límite de Chandrasekhar" y cuyo valor es aproximadamente de $1.4 \mathrm{M}_{\odot}$. El rol de la relatividad general en la modificación de la relación masa-radio para enanas blancas masivas (masas mayores a $\sim 1 \mathrm{M}_{\odot}$ ) fue discutido por primera vez por Kaplan (1949). Schatzman (1956) mostró que la inclusión de los decaimientos beta inversos en la ecuación de estado inducen una inestabilidad dinámica en la estructura de las enanas blancas más masivas que $\sim 1 \mathrm{M}_{\odot}$ (que tienen radios menores a $\sim 4 \times 10^{3} \mathrm{~km}$ ), que no puede ser compensada hasta que virtualmente todos los electrones y protones han sido convertidos en neutrones, es decir, hasta que se forma una estrella de neutrones.

La teoría básica del enfriamiento de las WDs, donde la energía radiada proviene de una disminución de su energía térmica interna $\left(E_{\mathrm{th}}\right)$, fue desarrollada por Mestel (1952), quien encontró que, bajo ciertas hipótesis (núcleo isotérmico, liberación de energía por compresión despreciable) la ecuación

$$
L(t)=-\frac{d E_{\mathrm{th}}}{d t}
$$

lleva a una simple relación

$$
t_{\text {cool }} \propto L^{-5 / 7}
$$

entre la edad y la luminosidad de una WD. Sin embargo esta teoría es demasiado simplificada y no incluye procesos físicos de importancia fundamental en la evolución de las WDs. El enfriamiento por neutrinos $\left(L_{\nu}\right)$ fue incluido por primera vez en los cálculos evolutivos durante la década de 1960 por Chin, Chiu \& Stothers (1966); Savedoff, Van Horn \& Vila (1969). Un efecto importante en la evolución de las WDs es la cristalización de sus interiores. El calor latente liberado durante este proceso, que da una contribución adicional a la liberación de energía térmica $\left(L_{\text {th }}\right)$, fue considerado por primera vez por Kovetz \& Shaviv (1970); Mestel \& Ruderman (1967); Van Horn (1968). Más recientemente, un tratamiento detallado de las capas externas no degeneradas ha incluido también la liberación de energía nuclear $\left(L_{\text {nuc }}\right)$ (Iben \& Tutukov, 1984; Koester \& Schönberner, 1986; Mazzitelli \& D’Antona, 1986). Además, la liberación de energía gravitatoria $\left(L_{g}\right)$ de las capas superficiales no degeneradas juega también un papel importante en las etapas tempranas y tardías de la evolución de las WDs (D'Antona \& Mazzitelli, 1989). De esta manera, actualmente la evolución de las WDs está determinada, desde el punto de vista de las contribuciones energéticas a la misma, por la ecuación más completa

$$
L(t)=L_{\mathrm{th}}+L_{g}+L_{\mathrm{nuc}}+L_{\nu}
$$

\subsection{Física general de las enanas blancas}

Las WDs representan el estado final de la evolución de la mayor parte de las estrellas. En efecto, las WDs son el resultado final, en general, de la evolución de estrellas de masas bajas e intermedias $\left(M \leq 8 \mathrm{M}_{\odot}\right)$ tanto en el caso de estrellas aisladas como en sistemas múltiples. Actualmente, la imagen básica que se tiene de la estructura de una enana blanca es en términos generales la siguiente: un núcleo de materia degenerada muy denso (las densidades promedio características son del orden de $\rho \approx 10^{6}-10^{7} \mathrm{~g} \mathrm{~cm}^{-3}$ ) que comprende alrededor del $99 \%$ de 
la masa total de la estrella, rodeado de una envoltura delgada de materia en condiciones no degeneradas, con densidades mucho menores.

La estructura de las enanas blancas es soportada, en su núcleo, por la presión ejercida por los electrones altamente degenerados (la existencia de las WDs es una confirmación espectacular del principio de exclusión de Pauli). La degeneración interna confiere a estas estrellas propiedades peculiares: la estructura mecánica queda esencialmente separada de la estructura térmica; la altísima conductividad térmica de la materia degenerada produce núcleos prácticamente isotérmicos; existe una relación inversa entre la masa y el radio de estas estrellas, que como límite extremo conduce a la existencia de un valor máximo en la masa que estos objetos pueden tener (masa límite de Chandrasekhar). Las reacciones nucleares juegan un papel secundario como fuente de energía de las enanas blancas. La energía radiada al espacio por las mismas es obtenida principalmente de la energía térmica acumulada en los iones no degenerados de su interior. A lo largo de su evolución la temperatura del núcleo de una enana blanca disminuye desde unos $10^{8} \mathrm{~K}$ iniciales hasta unos pocos $10^{6} \mathrm{~K}$ o incluso menos en los momentos en que estos objetos alcanzan luminosidades muy bajas, hacia el final de su evolución. Los efectos de la degeneración electrónica desaparecen en la envoltura de las WDs. Dichas envolturas son extremadamente delgadas (tanto en masa como en radio), sin embargo debido a las altas opacidades que presentan son extremadamente eficientes como reguladoras del flujo de energía emitido por la estrella. El transporte de energía en la envoltura de una WD puede ser tanto radiativo como convectivo, dependiendo de la fase evolutiva de la estrella (básicamente de su temperatura efectiva pero también de los detalles de la composición química externa).

La determinación observacional de las masas de las enanas blancas muestra una distribución de masas fuertemente centrada en $\approx 0.6 \mathrm{M}_{\odot}$ con colas extendiéndose hasta $\approx 0.15 \mathrm{M}_{\odot} \mathrm{y}$ $\approx 1.2 \mathrm{M}_{\odot}$. Los modelos de evolución estelar y formación de WDs predicen una relación entre la composición química del interior de las mismas y su masa total (existen, sin embargo, escenarios peculiares de formación de WDs que escapan a esta afirmación). Las enanas blancas de baja masa (masas $\leq 0.45 \mathrm{M}_{\odot}$ ) tienen un núcleo prácticamente puro de helio, aquellas con masas entre $0.45 \mathrm{M}_{\odot}$ у $1 \mathrm{M}_{\odot}$ aproximadamente (rango que incluye a la gran mayoría de las WDs) tienen núcleos formados por carbono y oxígeno (en proporciones no del todo bien determinadas por los modelos actuales), y en el caso de WDs de masas mayores es actualmente aceptado que sus núcleos están compuetos por oxígeno y neón.

Las envolturas de las WDs, como se dijo anteriormente, son las regiones exteriores al núcleo degenerado, y resultan delgadas tanto en radio como en masa. La composición química inicial de las envolturas depende de los detalles del proceso de formación de la WD, pero generalmente están formadas por hidrógeno y/o helio. Las altas gravedades superficiales de las WDs (log $g_{\text {sup }} \sim 7-8$ son valores típicos) hacen de la sedimentación gravitatoria un proceso muy eficiente en las envolturas de estas estrellas. De esta manera, aún cuando la composición inicial de la envoltura consista en una mezcla de hidrógeno y helio (más trazas de metales), la sedimentación gravitatoria lleva a la formación, en escalas de tiempo cortas desde el punto de vista de la evolución estelar, de envolturas casi puras de hidrógeno. Sin embargo, las observaciones muestran una realidad más compleja. Desde el punto de vista espectroscópico, las WDs se clasifican básicamente en dos tipos: DA, con atmósferas casi puras de hidrógeno, y DB con atmósferas ricas en helio (esta última clase incluye aproximadamente al $25 \%$ de las WDs conocidas). Existen muchas WDs que presentan peculiaridades en la composición química de sus atmósferas, que no pueden clasificarse como DA o DB. En la tabla 1.1 se presenta el esquema de clasificación espectral más común (Hansen \& Kawaler, 1989). Es interesante remarcar también que parece existir una "evolución" en el tipo espectral durante la evolución de las WDs. Es 
Table 1.1: Clasificación espectral de estrellas enanas blancas.

\begin{tabular}{cl}
\hline Tipo Espectral & Características \\
\hline DA & Líneas de H, sin He I o metales presentes \\
DB & Líneas de He solamente, sin H o metales presentes \\
DC & Espectros continuos sin líneas distinguibles \\
DO & Líneas intensas de He II; He I o H pueden estar presentes \\
DZ & Líneas metales solamente, sin H o He \\
DQ & Rasgos de C \\
\hline
\end{tabular}

decir, algunas WD DAs pueden transformarse en DBs y viceversa. Los motivos de estos cambios no están del todo claros, pero probablemente una combinación entre la convección que se produce en las envolturas de estas estrellas en ciertos rangos de temperaturas efectivas junto con la sedimentación gravitatoria sea responsable de los cambios en la composición química observada en las envolturas. También es probable que un WD sufra durante su vida episodios de acreción de material interestelar (p.ej. nubes moleculares encontradas mientras la WD se mueve en torno al centro galáctico).

Como se mencionó al comienzo de esta sección, las WDs son el resultado final de la evolución de estrellas con masas menores a $8 \mathrm{M}_{\odot}$ aproximadamente. Los procesos que llevan a la formación de las WDs son diversos y dependen, además de la masa inicial de la estrella progenitora, de la configuración en que ésta se encuentra (estrella sola, sistema binario). Describimos brevemente los mecanismos por los cuales, se cree, las WDs pueden ser formadas.

Consideremos primeramente el caso de una estrella aislada. Siguiendo a las etapas de combustión del hidrógeno y del helio en el núcleo de la estrella, la misma evoluciona (en un diagrama Hertzsprung-Russell) ascendiendo a lo largo de la rama asintótica de las gigantes (AGB), mientras la combustión nuclear tiene lugar en shells concéntricos alrededor de un núcleo de carbono-oxígeno inerte. Durante esta etapa la estrella mantiene una envoltura convectiva muy extendida y tiene dimensiones gigantes (radios del orden de cientos de radios solares son típicos en estas etapas), además sufre fuertes episodios de pérdida de masa. En el extremo más luminoso de la AGB, la estrella atraviesa una fase de pulsos térmicos, donde la ocurrencia reiterada de flashes termonucleares en el shell de helio provoca contracciones y expansiones alternadas de la estrella, incrementando la tasa de pérdida de masa. Los pulsos térmicos ocurren repetidamente hasta que por efectos de la pérdida de masa la envoltura se vuelve delgada y no puede mantenerse extendida. La estrella de esta manera se contrae, aleja de la AGB y comienza su evolución como núcleo de nebulosa planetaria, hacia el dominio de las enanas blancas. En este caso la WD resultante tendrá un núcleo compuesto por una mezcla de carbono y oxígeno (COWD) o, en casos extremos, de oxígeno y neón.

En el caso en que el progenitor se halla en un sistema binario (o múltiple), el mecanismo de formación de una WD dependerá de las características del sistema. Es decir, en aquellos casos en que la órbita sea extremadamente grande, puede considerarse desde el punto de vista de la evolución estelar que la estrella está aislada. Sin embargo, en aquellos casos en que esto no es así, la estrella puede llenar su lóbulo de Roche como resultado de la expansión que sufre durante determinadas etapas evolutivas. En este caso, se producen episodios de pérdida de masa por overflow del lóbulo de Roche. Si estos episodios ocurren cuando la estrella ya atravesó la fase de combustión de helio en su núcleo, la WD remanente tendrá nuevamente un núcleo caracterizado por una mezcla de carbono y oxígeno. Por el contrario, si el overflow del lóbulo de Roche ocurre cuando la estrella tiene todavía un núcleo de helio puede ocurrir (dependiendo de los detalles 
orbitales del sistema binario y de la masa inicial del progenitor) que la masa de la estrella progenitora sea reducida por debajo de la masa mínima necesaria para que el helio se encienda en su núcleo $\left(\sim 0.45 \mathrm{M}_{\odot}\right)$ antes de que el helio comience a quemarse. De esta manera resultará una WD remanente de baja masa caracterizada por un núcleo casi puro de helio (HeWD).

Los detalles de los procesos de formación de las WDs son importantes ya que de ellos dependen, no sólo la masa final y la composición química interna de la estrella, sino también las masas de las envolturas de hidrógeno y helio (en el caso de las COWDs). Estas cantidades resultan importantes en la evolución de las WDs. En particular, como se discute extensivamente en esta tesis, el espesor de la envoltura de hidrógeno en las HeWDs resulta clave para determinar las edades de enfriamiento de estas estrellas. En el caso de las COWDs, las propiedades pulsacionales de estas estrellas están en gran medida determinadas por los espesores de las envolturas de hidrógeno y de helio, como así también por la forma de sus interfaces.

\subsection{Motivación y objetivos}

Los últimos años han visto un desarrollo acelerado de la astronomía observacional. Los telescopios espaciales, y también una nueva generación de telescopios terrestres han producido observaciones de una calidad sin precedentes, arrojando una nueva luz sobre fenómenos previamente conocidos, pero también dando lugar a nuevos descubrimientos. Y las WDs no son una excepción en este sentido.

El interés en el estudio de las WDs ofrece un espectro amplio. Como remanentes más comunes de la evolución estelar, nuestra galaxia debe estar poblada por numerosas WDs, producto de más de 10 Gyr de evolución estelar galaćtica. Las enanas blancas más viejas en la vecindad solar son todavía visibles y pueden ser usadas, en conjunto con modelos teóricos de enfriamiento, para estimar la edad del disco galáctico. Observaciones recientes sugieren también la posibilidad de que una población muy vieja de WDs puebla el halo galáctico y puede contribuir apreciablemente a la materia oscura bariónica en la Vía Láctea. Esta población puede usarse para obtener una estimación independiente de la edad del halo. Por otro lado, la detección de secuencias de enfriamiento de enanas blancas en cúmulos estelares (tanto abiertos como globulares) permite obtener una estimación independiente de la edad de los mismos (ver en particular Richer et al. 1998, 1997; von Hippel 1998; von Hippel \& Gilmore 2000). Para un detalle de estos temas referimos al lector a Fontaine, Brassard \& Bergeron (2001).

Las WDs de baja masa, formadas por núcleos de helio, han sido observadas directamente sólo recientemente, aún cuando su existencia había sido predicha en la década de 1960 como resultado de la evolución binaria de estrellas de baja masa. El estudio de estas estrellas resulta muy interesante ya que el mismo involucra tanto a la teoría de las enanas blancas como así también la evolución de estrellas en sistemas binarios, donde tienen lugar fenómenos sumamente complejos como por ejemplo los episodios de transferencia de masa entre las componentes de los mismos. Además, dado que estas estrellas se encuentran muchas veces en compañía de púlsares de milisegundo, son un instrumento ideal para confrontar con las observaciones los modelos de estos púlsares. Es interesante notar también que las enanas blancas de baja masa están siendo detectadas tanto en el campo galáctico como así también en cúmulos globulares.

La heliosismología es hoy una herramienta fundamental en la comprensión de la estructura del Sol. De una manera equivalente, la astrosismología está brindándose también como un medio inigualable para "mirar" dentro de las estrellas. En efecto, dada la sensibilidad de las propiedades pulsacionales de las estrellas a los detalles de su estructura interna, la comparación 
del espectro pulsacional de las WDs y su comparación con modelos teóricos permite obtener información precisa sobre cosas tales como su composición química interna, velocidad de enfriamiento, etc. El conocimiento de la composición interna de las WDs, por ejemplo, permite a su vez estudiar los procesos mediante los cuales estas estrellas son formados, a través de modelos de evolución estelar. Por otro lado, dado que la velocidad de enfriamiento de las WDs depende de la energía total de que estas estrellas disponen y de los mecanismos mediante los cuales esta energía es disipada (radiación de energía térmica, perdida por emisión de neutrinos, emisión de otro tipo de partículas tales como los axiones), la astrosismología promete aportar importantes contribuciones a diversos campos de investigación tales como la física de partículas y la cosmología.

El gran desarrollo de las observaciones en cuanto a la cantidad y la calidad de los nuevos datos que éstas aportan, plantea muchas nuevas preguntas e incógnitas y revela que, desde el punto de vista teórico, la estructura y evolución de las enanas blancas es un tema que dista enormemente de ser comprendido en detalle. En base a este convencimiento, el objetivo general de esta tesis es presentar cálculos detallados de la evolución y estructura de estrellas enanas blancas de masas bajas e intermedias.

Desde el punto de vista del estudio de las WDs de baja masa (con núcleos de helio), el interés se centra en obtener modelos de la evolución "completa" de estos objetos, es decir, comenzando con sus progenitores desde la secuencia principal hasta el momento en que se vuelven "invisibles" debido a sus bajas luminosidades superficiales, al final de su evolución.

En el caso de las WDs de masas intermedias (con núcleo de carbono-oxígeno), el principal objetivo buscado es la obtención de modelos detallados de su estructura interna, en particular en lo que hace a la estratificación química interna de las mismas. Esto permitirá desarrollar modelos pulsacionales para estas estrellas en base a modelos más realistas que los usados hasta el momento en las investigaciones sobre este tema, necesarios para una correcta interpretación de las actuales (y futuras) observaciones de enanas blancas pulsantes.

Para alcanzar los objetivos mencionados, es decir, la obtención de modelos de enanas blancas con el mayor detalle posible, se han tenido en cuenta en el cálculo de los modelos presentados en esta tesis diversos procesos físicos generalmente no tenidos en cuenta por considerarse, erróneamente en muchos casos, de importancia secundaria. Una breve mención de los mismos y su importancia se presentan a continuación.

Durante la evolución de las WDs, en particular para las de baja masa, se incluye un tratamiento detallado de la atmósfera de las mismas. Esto permite por un lado un tratamiento realista de las condiciones de borde externas para la resolución de las ecuaciones de estructura y evolución estelar y por otro la obtención de cantidades tales como magnitudes absolutas, índices de color y correcciones bolométricas, que permiten una comparación directa con las observaciones. Asímismo se presenta un análisis pormenorizado de la influencia de los procesos de difusión microscópica en la evolución de las WDs, procesos generalmente despreciados en las investigaciones sobre estas estrellas. La evolución de las WDs con núcleos de helio resulta profundamente afectada por los efectos de la difusión. En esta tesis se muestra que la difusión no es bajo ningún punto de vista un mecanismo físico secundario. Por otro lado, en el caso de enanas blancas de carbono y oxígeno, se muestra cómo los perfiles químicos internos de estos objetos son modelados por efectos de la difusión. Esto tiene importantes implicancias en los espectros pulsacionales de las enanas blancas. Un estudio detallado de las enanas blancas pulsantes está fuera del alcance de esta tesis, sin embargo se presentan algunos primeros resultados de propiedades oscilatorias obtenidos por nuestro grupo de investigación en el marco del modelado evolutivo considerado en esta tesis. Por otro lado, la estructura de las WDs no 
puede considerarse como algo aislado de la historia previa de estos objetos, es decir, de los procesos que llevan a su formación. Esto nos lleva a considerar también la evolución previa de los progenitores de las enanas blancas. En este sentido, la evolución de estrellas de baja masa en sistemas binarios, que da origen a enanas blancas de helio, es estudiada en detalle partiendo de modelos de secuencia principal. En el caso de las WDs de masas intermedias, la evolución de sus progenitores a lo largo de la rama asintótica de las gigantes, etapa en la cual los perfiles químicos internos iniciales de las enanas blancas son formados, es también seguida en detalle. 


\section{Capítulo 2}

\section{El código de evolución estelar}

En este capítulo se introducen brevemente las ecuaciones que describen la evolución estelar. Así mismo se presenta de manera resumida el tratamiento numérico empleado para resolver las ecuaciones que describen el problema mediante el uso de un código de evolución estelar. Finalmente hay una descripción de los ingredientes físicos incluidos en el código de evolución (opacidades, tasas de reacciones nucleares, ecuación de estado, tasas de emisión de neutrinos, etc.). Este capítulo es una descripción general, que intenta abarcar aquellas características del código que son comunes a todos los cálculos presentados en la tesis. El modelado de estrellas de diferentes características, sin embargo, involucra la adopción de tratamientos numéricos particulares, como así también la inclusión de mecanismos físicos que pueden ser relevantes en algunos casos y no en otros. Estos detalles particulares serán descriptos oportunamente cuando se presenten los cálculos correspondientes.

Los cálculos de evolución estelar presentados en esta tesis han sido realizados considerando a las estrellas como objetos esféricos. Esta hipótesis limita el estudio de una variedad de fenómenos muy interesantes. Sólo por enumerar algunos de ellos pueden mencionarse la distribución de momento angular en el interior estelar, la mezcla de composición química debido a corrientes meridionales, la influencia de campos magnéticos en la estructura estelar, etc. Sin embargo, los estudios teóricos y las simulaciones numéricas de estructura y evolución estelar bajo la hipótesis de simetría esférica han mostrado a lo largo de los años ser una excelente aproximación en la gran mayoría de los casos gracias a los numerosos éxitos en el estudio de las estrellas. A continuación se presentan las ecuaciones que determinan el estudio de la evolución y estructura estelar en la aproximación de simetría esférica y también una descripción de los métodos numéricos empleados en el código evolutivo para la resolución de dichas ecuaciones.

\subsection{Ecuaciones de estructura y evolución}

Las ecuaciones básicas que determinan la estructura y evolución estelar son bien conocidas y no es necesario hacer una descripción pormenorizada de ellas en esta tesis. Sin embargo, vale la pena presentar una breve discusión de algunos puntos importantes. Considerando la masa interna $m$ como variable independiente (variable lagrangiana) las ecuaciones son (Kippenhahn \& Weigert, 1990) 


$$
\begin{aligned}
\frac{\partial r}{\partial m} & =\frac{1}{4 \pi r^{2} \rho} \\
\frac{\partial P}{\partial m} & =-\frac{G m}{4 \pi r^{4}}-\frac{1}{4 \pi r^{4}} \frac{\partial^{2} r}{\partial t^{2}} \\
\frac{\partial l}{\partial m} & =\epsilon_{\text {nuc }}-\epsilon_{\nu}-c_{P} \frac{\partial T}{\partial t}+\frac{\delta}{\rho} \frac{\partial P}{\partial t} \\
\frac{\partial T}{\partial m} & =-\frac{G m T}{4 \pi r^{4} P} \nabla
\end{aligned}
$$

Las cantidades involucradas en estas ecuaciones son: $m$ es la variable masa (independiente), $t$ el tiempo, $r=r(m, t)$ la distancia desde el centro de la estrella, $P=P(m, t)$ es la presión, $l=l(m, t)$ es la luminosidad, $T=T(m, t)$ la temperatura, $\rho$ es la densidad de masa, $\epsilon_{n u c}$ la liberación de energía nuclear por unidad de masa y de tiempo, $\epsilon_{\nu}$ la pérdida de energía por emisión de neutrinos por unidad de masa y de tiempo, $c_{P}$ el calor específico a presión constante por unidad de masa, $\delta=\left(\frac{\partial \ln \rho}{\partial \ln T}\right)_{P}$ y $\nabla=\left(\frac{\partial \ln T}{\partial \ln P}\right)$ el gradiente adimensional de temperatura. Además $G$ es la constante de gravitación universal, $c$ la velocidad de la luz en el vacío, $a$ la constante de densidad de radiación y $t$ el tiempo.

Las tres primeras ecuaciones expresan leyes físicas bien conocidas: la conservación de la masa, la ecuación de movimiento y la conservación de la energía respectivamente. La cuarta ecuación determina el transporte de energía dentro del interior estelar.

Consideremos la ecuación de movimiento (2.2). El segundo término del miembro derecho es proporcional a la aceleración del elemento de masa considerado y es relevante cuando la estructura estelar no se encuentra en equilibrio hidrostático. Sin embargo, frente a perturbaciones pequeñas del equilibrio hidrostático, la estructura estelar se reajusta para reestablecer el equilibrio en una escala de tiempo del orden de

$$
\tau_{h i d r} \approx\left(\frac{R^{3}}{G M}\right)^{1 / 2}
$$

En el caso del Sol esta escala de tiempo es del orden de decenas de minutos, para estrellas gigantes $\left(M \approx M_{\odot}, R \approx 100 R_{\odot}\right)$ esta escala resulta ser del orden de días, y en enanas blancas del orden de segundos. En cualquiera de los casos mencionados, y que son representativos de aquéllos que nos interesan en esta tesis, la escala de tiempo hidrostática $\tau_{\text {hidr }}$ resulta mucho más corta que las escalas de tiempo en las cuales se producen cambios en la estructura estelar. Esto permite realizar una importante simplificación en las ecuaciones de estructura porque el término de aceleración en la ecuación (2.2) resulta despreciable, por lo que esta ecuación puede ser aproximada por la de equilibrio hidrostático

$$
\frac{\partial P}{\partial m}=-\frac{G m}{4 \pi r^{4}}
$$

que es la que ha sido utilizada en todos los cálculos presentados en esta tesis en lugar de la ec. 2.2 .

En la ecuación de transporte de energía (2.4) una cantidad fundamental es $\nabla$, que determina la estratificación de la temperatura en el interior estelar. Cuando el transporte de energía ocurre 
por radiación o conducción, $\nabla$ debe ser reemplazado por el gradiente radiativo $\nabla_{\text {rad }}$, definido por

$$
\nabla_{\text {rad }}=\frac{3}{16 \pi a c G} \frac{\kappa l P}{m r^{4}} .
$$

donde $\kappa$ es la opacidad radiativa y/o conductiva. En el caso en que el transporte de energía ocurre por convección, $\nabla$ debe obtenerse a partir de una teoría apropriada de convección. En el caso de las zonas más internas de una estrella la convección, en caso de estar presente, es prácticamente adiabática, de modo que $\nabla=\nabla_{a d}$ en un excelente grado de aproximación. Sin embargo en otras condiciones, como por ejemplo la zona convectiva externa de una gigante roja, hay un apreciable grado de superadiabaticidad y por lo tanto $\nabla$ se aparta apreciablemente de $\nabla_{a d}$. En estos casos debe recurrirse indefectiblemente a una teoría de convección no adiabática (p.ej. teoría de la longitud de mezcla (MLT)).

Resumiendo, el conjunto de ecuaciones que determinan la estructura y también la evolución estelar que ha sido empleado en los cálculos presentados en esta tesis es

$$
\begin{aligned}
& \frac{\partial r}{\partial m}=\frac{1}{4 \pi r^{2} \rho} \\
& \frac{\partial P}{\partial m}=-\frac{G m}{4 \pi r^{4}} \\
& \frac{\partial l}{\partial m}=\epsilon_{\mathrm{nuc}}-\epsilon_{\nu}-c_{P} \frac{\partial T}{\partial t}+\frac{\delta}{\rho} \frac{\partial P}{\partial t} \\
& \frac{\partial T}{\partial m}=-\frac{G m T}{4 \pi r^{4} P} \nabla \quad \text { donde } \quad \nabla= \begin{cases}\nabla_{\mathrm{rad}} & \text { (trans.radiativo/conductivo) } \\
\nabla_{\text {conv }} & \text { (trans.convectivo) }\end{cases}
\end{aligned}
$$

El conjunto de ecuaciones (2.8-2.11), junto con las ecuaciones que describen los cambios en la composición química (y que son descriptas en la sección 2.3) son las determinan la evolución y estructura estelar.

\subsection{Tratamiento numérico de las ecuaciones de estruc- tura}

El código de evolución estelar (CEELP) empleado para esta tesis ha sido desarrollado completamente en el Observatorio de La Plata. Originalmente desarrollado por el Dr. Omar Benvenuto y posteriormente adaptado para el estudio de estrellas enanas blancas por el Dr. Leandro Althaus, ha sido utilizado previamente en numerosos trabajos de evolución estelar, particularmente en cálculos evolutivos de enanas blancas (Althaus \& Benvenuto, 1997, 1998; Benvenuto \& Althaus, 1998; Althaus \& Benvenuto, 2000).

El método empleado para la resolución del sistema de ecuaciones de estructura (2.8-2.11) está basado en un esquema tipo Henyey. Este esquema es el más difundido en los códigos de evolución estelar y para una descripción exhaustiva sobre el mismo referimos al lector a Kippenhahn, Weigert \& Hofmeister (1967). La implementación de este esquema en el CEELP es a través de una linealización implícita del sistema de ecuaciones y el sistema algebraico resultante se resuelve entonces iterativamente (método Newton-Raphson). Dado que este esquema es prácticamente el estándar en los códigos de evolución estelar, solamente se mencionarán algunas características particulares del tratamiento de las ecuaciones de estructura en el CEELP. 
Las ecuaciones de estructura en su forma (2.8-2.11) responden a la elección de una variable lagrangiana como variable independiente. La elección implementada en el CEELP es tomar como variable independiente a la masa fraccionaria $m_{r}=m / M_{*}$, donde $M_{*}$ es la masa total de la estrella. Entonces, la resolución de las ecuaciones de estructura permite obtener las cantidades de estructura $T=T\left(m_{r}\right), P=P\left(m_{r}\right), r=r\left(m_{r}\right)$ y $l=l\left(m_{r}\right)$ a un tiempo dado. Vale la pena remarcar que las demás cantidades que aparecen en las ecs.(2.8-2.11) son funciones de $T, P, r$, $l$ y de la composición química, que representaremos genéricamente como $X_{i}$. Es decir que, dada una composición química, las ecuaciones de estructura representan un sistema de ecuaciones bien determinado. La evolución temporal de la composición química $X_{i}$ se describirá en detalle en la sección siguiente. Por ahora asumamos que $X_{i}$ es conocida de modo que sólo debemos preocuparnos por $T, P, r$ y $l$.

La estabilidad de los cálculos numéricos es mejorada de manera notable si se realizan cambios de variables apropiados. En el CEELP el cambio de variables implementado es el siguiente

$$
\begin{aligned}
\xi & =\ln \left(1-m_{r} / M_{*}\right) & & \\
\theta & =\ln T / T_{o} & & T_{o} \equiv 10^{6} \mathrm{~K} \\
p & =\ln P / P_{o} & & P_{o} \equiv 10^{15}{\mathrm{din} \mathrm{cm}^{-2}} \\
x & =\ln r / r_{o} & & r_{o} \equiv 10^{10} \mathrm{~cm}^{-1} \\
\lambda & =l / \Lambda L_{o} & & L_{o} \equiv 10^{33} \mathrm{erg} \mathrm{s}^{-1}
\end{aligned}
$$

De esta manera $\xi$ es la variable independiente usada en el CEELP y $\theta, p, x, \lambda$ son las variables dependientes. $T_{o}, P_{o}, r_{o}$ y $L_{o}$ son factores de escala. La cantidad $\Lambda$ es también un factor de escala que se reajusta automáticamente durante el cálculo de modo de mantener el valor absoluto de $\lambda$ por debajo de 10. Es interesante notar que el cambio de variable que se adopte en el caso de la luminosidad debe permitir la existencia de valores negativos de esta cantidad. Esto es físicamente posible por ejemplo en los casos en que la estrella es enfriada por la emisión de neutrinos (ver ec. 2.10), o en regiones por debajo de un shell donde la combustión nuclear ocurre de manera violenta, lo que da lugar a un gradiente de temperatura negativo, es decir que la temperatura no necesariamente aumenta hacia el centro de la estrella y existe un flujo de energía hacia el interior de la misma.

El cambio logarítmico de variables para $T, P$ y $r$ ofrece una ventaja muy importante desde el punto de vista numérico. Para ejemplificarla consideremos la temperatura $T$. Con un supraíndice $n$ denotamos las cantidades referidas a un instante de tiempo $t$ y por $n+1$ las correspondientes al paso temporal siguiente $(t+\Delta t)$. Entonces

$$
T^{(n+1)}=T^{(n)}+\Delta T=T^{(n)}\left(1+\frac{\Delta T}{T^{(n)}}\right)
$$

de manera que si introducimos el cambio de variable (2.13) resulta

$$
\theta^{(n+1)}=\theta^{(n)}+\ln \left(1+\frac{\Delta T}{T^{(n)}}\right)=\theta^{(n)}+\ln \left(1+u_{\theta}\right) .
$$

Durante el proceso iterativo la variable que se itera es entonces la diferencia $u_{\theta}=\frac{\Delta T}{T^{(n)}}$ en vez de $\theta^{(n+1)}$ (de un modo análogo se introducen $u_{x} \mathrm{y} u_{p}$ ) lo cual resulta muy ventajoso para evaluar derivadas temporales. Las únicas derivadas de este tipo que aparecen son las derivadas 
temporales de la temperatura y la presión en la ecuación de la luminosidad (2.10). Siguiendo con el ejemplo de la temperatura tenemos

$$
\frac{\partial T}{\partial t}=T_{o} \frac{\partial}{\partial t} \exp \theta=T_{o} \exp \theta \frac{\partial \theta}{\partial t}
$$

y al linealizar la derivada temporal resulta

$$
\frac{\partial T}{\partial t} \approx T_{o} \exp \theta\left(\frac{\theta^{(n+1)}-\theta^{(n)}}{\Delta t}\right)
$$

Sin embargo, usando (2.18) esta derivada puede reescribirse como

$$
\frac{\partial T}{\partial t} \approx T_{o} \exp \theta \frac{\ln \left(1+u_{\theta}\right)}{\Delta t} .
$$

La gran ventaja de introducir las variables logarítmicas y usar $u_{\theta}, u_{p}$ y $u_{x}$ como variables a ser iteradas (como se sugiere en Kippenhahn, Weigert \& Hofmeister 1967), es que permite evaluar las derivadas temporales como se muestra en la ecuación (2.21), es decir, sin tener que efectuar la resta de dos cantidades que pueden ser muy parecidas (como en 2.20). Esto último puede llevar a errores de redondeo que pueden afectar la convergencia global de la integración llegando a imposibilitar el cálculo de ciertas etapas evolutivas dificultosas desde el punto de vista numérico (p.ej. los pulsos térmicos en la rama asintótica de las gigantes). Los cambios de variable implementados son finalmente junto con las ecuaciones (2.12-2.16) son

$$
\begin{aligned}
\theta^{(n+1)} & =\theta^{(n)}+\ln \left(1+u_{\theta}\right) \\
p^{(n+1)} & =p^{(n)}+\ln \left(1+u_{p}\right) \\
x^{(n+1)} & =x^{(n)}+\ln \left(1+u_{x}\right) \\
l^{(n+1)} & =l^{(n)}+u_{l}
\end{aligned}
$$

de manera que las cantidades calculadas durante el proceso iterativo son $u_{\theta}, u_{p}, u_{x} \mathrm{y} u_{l}$.

\subsection{Ecuaciones de cambios de composición química}

Esquemáticamente, si $n_{i}$ es el número de partículas por unidad de volumen de la especie $i$, el cambio de la composición química en un elemento de masa dado puede escribirse como

$$
\frac{\partial n_{i}}{\partial t}=\left(\frac{\partial n_{i}}{\partial t}\right)_{n u c}+\left(\frac{\partial n_{i}}{\partial t}\right)_{m i x}, \quad i=1, \ldots \ldots, I
$$

donde el primer término del miembro derecho representa el cambio producido como resultado de las reacciones nucleares, y el segundo el cambio producido por diferentes mecanismos de mezcla: convección, semiconvección, difusión microscópica, inestabilidad Rayleigh-Taylor. Por cada especie química relevante hay una ecuación (2.26) y todas están acopladas entre sí, es decir que en realidad (2.26) representa un sistema de $I$ ecuaciones diferenciales acopladas ( $I$ es el número total de especies nucleares consideradas).

Consideremos primero el término debido a las reacciones nucleares. Una especie nuclear dada puede ser creada y destruida a través de numerosas reacciones nucleares con otras especies. Si $\langle v \sigma\rangle_{i j}$ denota la tasa de reacciones nucleares por par de partículas entre las especies $i$ y $j$, 
$\mathrm{y}\langle v \sigma\rangle_{k l}$ la correspondiente a las reacciones entre partículas $k$ y $l$ que producen partículas $i$ tenemos

$$
\left(\frac{\partial n_{i}}{\partial t}\right)_{n u c}=-\sum_{j}\langle v \sigma\rangle_{i j} n_{i} n_{j}+\sum_{k, l}\langle v \sigma\rangle_{k l} n_{k} n_{l}
$$

La primera sumatoria da cuenta de las reacciones que destruyen partículas de la especie $i$, mientras que la segunda lo hace de las reacciones que crean partículas de esta especie. Debe tenerse en mente que ésta es una representación esquemática, ya que las reacciones nucleares no involucran necesariamente dos especies diferentes, sin embargo es suficiente para ilustrar el esquema empleado para la solución del sistema de ecuaciones 2.26 (para más detalles ver Arnett 1996).

La segunda sumatoria en la ecuación (2.26) representa una variedad muy amplia de procesos de mezcla que pueden modificar la composición química en una zona de la estrella, de los cuales el más conocido es sin duda la convección. Es interesante notar, sin embargo, que los diferentes procesos de mezcla pueden modelarse como procesos de difusión de la composición química con coeficientes de difusión apropiados para cada proceso. Entonces, asumiendo simetría esférica, podemos escribir

$$
\left(\frac{\partial n_{i}}{\partial t}\right)_{m i x}=\frac{1}{r^{2}} \frac{\partial}{\partial r}\left(r^{2} D_{m i x} \frac{\partial n_{i}}{\partial r}\right)
$$

donde $D_{m i x}$ representa el coeficiente de difusión determinado por el mecanismo de mezcla considerado. Es importante notar que debido a la linealidad de estas ecuaciones con respecto a $D_{\text {mix }}$, si dos a más mecanismos de mezcla operan simultáneamente sus efectos pueden ser sumados directamente definiendo $D_{m i x}=\sum_{\text {proc }} D_{\text {proc }}$.

La ecuación (2.26) que determina la evolución temporal de la composición química queda reescrita entonces como

$$
\frac{\partial n_{i}}{\partial t}=-\sum_{j}\langle v \sigma\rangle_{i j} n_{i} n_{j}+\sum_{k, l}\langle v \sigma\rangle_{k l} n_{k} n_{l}+\frac{1}{r^{2}} \frac{\partial}{\partial r}\left(r^{2} D_{m i x} \frac{\partial n_{i}}{\partial r}\right) \quad i=1, \ldots \ldots, I
$$

y junto con el conjunto de ecuaciones (2.8-2.11) son las ecuaciones que determinan el problema de la estructura y evolución estelar.

\subsection{Tratamiento numérico de las ecuaciones de cambio de composición química}

Los cambios de la composición química en un modelo estelar, dados por el conjunto de ecuaciones (2.29), no son tratados en los códigos de evolución estelar de manera completamente autoconsistente con los cambios en la estructura estelar. En efecto, como se dijo en la sección 2.2, en el momento de integrar las ecuaciones de estructura en un paso temporal la composición química se supone conocida. Una vez obtenidas $T, P, r$ y $l$ al tiempo $n+1$ se avanza la composición química mediante la integración de las ecuaciones $(2.29)$ al nuevo tiempo $(n+2)$. Luego se avanzan las cantidades de estructura y así sucesivamente. Esquemáticamente, el mecanismo se ilustra en la figura 2.1. La resolución simultánea de las ecuaciones de estructura y de composición química implica un enorme costo computacional y no aparece como imprescindible (aunque sí deseable) si el paso temporal se mantiene suficientemente pequeño como 
$\left.\ldots X_{i}^{(n-1)} \rightarrow \begin{array}{c}T^{(n-1)}, P^{(n-1)} \\ r^{(n-1)}, l^{(n-1)}\end{array} \rightarrow X_{i}^{n} \rightarrow \begin{array}{c}T^{n}, P^{n} \\ r^{n}, l^{n}\end{array}\right) \rightarrow X_{i}^{(n+1)} \rightarrow \begin{gathered}T^{(n+1)}, P^{(n+1)}, \\ r^{(n+1)}, l^{(n+1)}\end{gathered} \ldots$

Figura 2.1: Solución alternada de los cambios en composición química y variables de estructura.

para que tanto las variables de estructura como la composición química no cambien demasiado en cada paso de integración. Por otro lado, el requerimiento de cambios pequeños en cada paso evolutivo es necesario para que la linealización de las ecuaciones se mantenga como una buena aproximación.

Al contrario que con las ecuaciones de estructura (2.8-2.11), el tratamiento numérico de las ecuaciones (2.29) no ha sido el mismo en todos los cálculos presentados en esta tesis. En función de las diferentes etapas evolutivas involucradas, diferentes procesos físicos adquieren o pierden relevancia para determinar la composición química y por lo tanto es en función de las necesidades específicas de cada cálculo cómo se han tratado las ecuaciones (2.29).

Consideremos primero el caso de una zona de la estrella donde el transporte ocurre por radiación o conducción. En estas condiciones el fluido es dinámicamente estable por lo que no existen mecanismos de mezcla de la composición química por movimientos macroscópicos del fluido. Dejando de lado los efectos de la difusión microscópica, los cambios en la composición química se deben entonces sólo a la ocurrencia de las reacciones nucleares. En este caso, las ecuaciones (2.29) se reducen a

$$
\frac{\partial n_{i}}{\partial t}=-\sum_{j}\langle v \sigma\rangle_{i j} n_{i} n_{j}+\sum_{k, l}\langle v \sigma\rangle_{k l} n_{k} n_{l} \quad i=1, \ldots \ldots, I .
$$

Seguimos el tratamiento presentado por Arnett \& Truran (1969). Para eso introducimos una nueva cantidad $Y_{i}$ definida por

$$
Y_{i}=\frac{n_{i}}{N_{A} \rho}
$$

donde $N_{A}$ es el número de Avogadro, de manera que las ecuaciones (2.30) pueden reescribirse como

$$
\frac{\partial Y_{i}}{\partial t}=-\sum_{j} N_{A} \rho\langle v \sigma\rangle_{i j} Y_{i} Y_{j}+\sum_{k, l} N_{A} \rho\langle v \sigma\rangle_{k l} Y_{k} Y_{l}
$$

e introduciendo $\lambda_{i j}=N_{A} \rho\langle v \sigma\rangle_{i j}$ finalmente resulta

$$
\frac{\partial Y_{i}}{\partial t}=-\sum_{j} \lambda_{i j} Y_{i} Y_{j}+\sum_{k, l} \lambda_{k l} Y_{k} Y_{l} \quad i=1, \ldots \ldots, I .
$$

Es útil remarcar que la relación entre $Y_{i}$ y $X_{i}$ (la fracción de masa, o más apropiadamente la fracción de nucleones) es simplemente $Y_{i}=X_{i} / A_{i}$, donde $A_{i}$ es la masa atómica de la especie nuclear considerada.

Conocidas las cantidades termodinámicas $\rho$ y $T$, y las abundancias al tiempo $t^{(n)}$, debemos integrar el sistema de ecuaciones representado por (2.33) un paso temporal, hasta $t^{(n+1)}=$ $t^{(n)}+\Delta t$. Para que el método numérico resulte estable sin necesidad de utilizar pasos temporales 
extremadamente pequeños, la linealización debe ser implícita. Para esto escribimos $Y_{i}^{(n+1)}=$ $Y_{i}^{(n)}+\Delta Y_{i}$ de modo que el producto $Y_{i}^{(n+1)} Y_{j}^{(n+1)}$ puede escribirse como

$$
Y_{i}^{(n+1)} Y_{j}^{(n+1)}=Y_{i}^{(n+1)} Y_{j}^{(n)}+Y_{i}^{(n)} Y_{j}^{(n+1)}-Y_{i}^{(n)} Y_{j}^{(n)}+\mathcal{O}\left(\Delta Y^{2}\right)
$$

de donde resulta el sistema de ecuaciones algebraicas

$$
\begin{aligned}
\frac{Y_{i}^{(n+1)}-Y_{i}^{(n)}}{\Delta t}= & -\sum_{j} \lambda_{i j}\left(Y_{i}^{(n+1)} Y_{j}^{(n)}+Y_{i}^{(n)} Y_{j}^{(n+1)}-Y_{i}^{(n)} Y_{j}^{(n)}\right)+ \\
& +\sum_{k, l} \lambda_{k l}\left(Y_{k}^{(n+1)} Y_{l}^{(n)}+Y_{k}^{(n)} Y_{l}^{(n+1)}-Y_{k}^{(n)} Y_{l}^{(n)}\right)+\mathcal{O}\left(\Delta Y^{2}\right), \\
& \quad \operatorname{para} i=1, \ldots . ., I
\end{aligned}
$$

en el cual las incógnitas son las cantidades $Y_{i}^{(n+1)}$. El paso temporal $\Delta t$ debe mantenerse lo suficientemente pequeño como para que el término de orden cuadrático en los incrementos sea despreciable. Generalmente es aconsejable dividir el paso utilizado en la integración de las ecuaciones de estructura en varios pasos para la integración de las ecuaciones (2.35) de modo de mantener $\Delta Y^{2}$ pequeño sin necesidad de calcular una excesiva cantidad de modelos evolutivos. En los cálculos presentados en esta tesis, el paso evolutivo fue dividido por un factor entre 5 y 10 para resolver $(2.35)$.

Consideremos ahora el caso en el que hay mezcla por convección. Además consideremos el caso en el que la mezcla es muy eficiente en toda la zona convectiva. Las escalas de tiempo asociadas con la convección son generalmente mucho más cortas que las escalas de tiempo nucleares, es decir aquellas sobre las que la abundancia de alguna especie nuclear cambia apreciablemente por efectos de las reacciones nucleares. Por ejemplo, en el interior de una estrella con un núcleo convectivo donde se fusiona el hidrógeno, la escala de tiempo nuclear puede variar desde unos cientos de miles de años hasta unos cientos de millones de años, mientras que la convección opera en escalas temporales de a lo sumo algunos años. En cualquier caso, la convección es generalmente varios órdenes de magnitud más rápida que la combustión nuclear. Esto lleva a considerar una hipótesis simplificatoria que es considerar a la región convectiva de una estrella como químicamente homogénea. Bajo esta hipótesis, la evolución de la composición química de una región convectiva se calcula suponiendo que la mezcla convectiva es tan eficiente que la región convectiva se mantiene químicamente homogénea y que las tasas efectivas de las reacciones nucleares son un promedio de estas tasas en toda la región. De esta manera definimos una tasa efectiva para una reacción nuclear como

$$
\lambda_{i j, \text { eff }}=\frac{1}{M_{Z C}} \int_{M_{Z C}} \lambda_{i j} d m
$$

de modo que (2.33) se reescribe como

$$
\frac{\partial Y_{i}}{\partial t}=-\sum_{j} \lambda_{i j, e f f} Y_{i} Y_{j}+\sum_{k, l} \lambda_{k l, \text { eff }} Y_{k} Y_{l} \quad i=1, \ldots \ldots, I,
$$

y esta ecuación determina el cambio de abundancia en toda la zona convectiva que, de este modo, permanece homogénea durante todo el cálculo. Bajo ciertas condiciones, sin embargo, la mezcla "instantánea" no es una buena aproximación. Esto puede ocurrir por diversos motivos. Por un lado puede ocurrir que la convección sea ineficiente en ciertas regiones de una estrella o durante ciertas etapas evolutivas donde las escalas de tiempo nucleares son reducidas de manera notable 
(p.ej. durante un flash termonuclear). Puede ocurrir también que la mezcla sea eficiente desde el punto de vista de la evolución estelar pero nos interesa estudiar la evolución de cierta especie nuclear cuyos cambios temporales por efecto de las reacciones nucleares sean muy rápidos. Un ejemplo de esto lo constituye la abundancia de neutrones durante los pulsos térmicos en la rama asintótica de las gigantes. Aquí los neutrones son muy importantes desde el punto de vista de la nucleosíntesis pues su abundancia determina la importancia del proceso lento de captura de neutrones, mecanismo responsable de gran parte de la existencia de elementos más pesados que el hierro. Los neutrones tienen una vida media del orden de minutos por lo que si se encuentran en una región donde hay convección un tratamiento instantáneo de la misma es a todas luces una aproximación burda al problema y seguramente lleve a conclusiones erróneas. Finalmente, cabe mencionar que existen otros mecanismos de mezcla, como la semiconvección, que son mucho menos eficientes que la convección y por lo tanto ocurren en escalas de tiempo que semejarán más a las escalas nucleares, por lo que un tratamiento simultáneo de la mezcla y las reacciones nucleares debe ser utilizado para su correcto estudio.

Sobre algunos de estos procesos volveremos más adelante, principalmente en relación a la evolución de estrellas de masas intermedias durante la etapa de pulsos térmicos.

\subsection{Física constitutiva}

Para poder resolver las ecuaciones de estructura (2.8-2.11) y las de composición química (2.29) es necesario conocer ciertas cantidades en función de las variables de estructura y la composición química del material estelar. Para ser más específicos, deben conocerse

$$
\begin{aligned}
\rho & =\rho\left(T, P, X_{i}\right) & \nabla_{a d} & =\nabla_{a d}\left(T, P, X_{i}\right) \\
\delta & =\delta\left(T, P, X_{i}\right) & c_{P} & =c_{P}\left(T, P, X_{i}\right) \\
\epsilon_{\mathrm{nuc}} & =\epsilon_{\mathrm{nuc}}\left(T, P, X_{i}\right) & \epsilon_{\nu} & =\epsilon_{\nu}\left(T, P, X_{i}\right) \\
\kappa & =\kappa\left(T, P, X_{i}\right) & \langle\sigma v\rangle_{i j} & =\langle\sigma v\rangle_{i j}(T, \rho) .
\end{aligned}
$$

Las cantidades $\rho, \nabla_{a d}, \delta$ y $c_{P}$ deben calcularse a través de una ecuación de estado para la materia estelar. Para esta tesis, varias EOS han sido incluidas y utilizadas alternativamente en el CEEPL. En el caso de materia no degenerada y donde las correcciones coulombianas son despreciables para determinar el estado de ionización parcial de la materia, se ha hecho uso de una ecuación de estado correspondiente a un gas ideal en la cual el grado de ionización parcial de la materia se obtiene resolviendo la ecuación de Saha para un gas compuesto de hidrógeno y helio. Las correcciones debidas a la presencia de metales es en este caso despreciable ya que cuando hay ionización parcial en situaciones reales, los elementos pesados son solamente trazas e influyen de modo despreciable a la EOS. La EOS para materia degenerada incluye tanto los casos de degeneración débil $|\alpha| \ll 1$ como fuerte $|\alpha| \gg 1$ como fuerte. Aquí $-\alpha$ es el potencial químico de los electrones en unidades de $k_{B} T$ ( $k_{B}$ es la constante de Boltzmann). Es bien sabido que la EOS para materia degenerada está planteada en término de integrales conocidas como integrales de Fermi (Chandrasekhar, 1939; Clayton, 1968). Estas integrales no tienen solución analítica por lo que en el CEELP se recurre a desarrollos en serie apropiados para los diferentes regímenes de degeneración (el detalle de los desarrollos puede encontrarse en Clayton 1968). La EOS para materia degenerada incorpora también contribuciones iónicas e interacciones Coulombianas. En todos los casos se incorporan los efectos en las cantidades termodinámicas debidos a la presencia de una gas de radiación. En el caso en que la ionización parcial ocurre bajo condiciones en que las interacciones Coulombianas son relevantes se usa una versión actualizada de la EOS de Magni \& Mazzitelli (1979). 
La tasa de emisión de neutrinos incluye los procesos de pares de neutrinos, foto-neutrinos, plasma y Bremsstrahlung siguiendo los lineamientos de Itoh et al. (1996).

Las opacidades radiativas son la versión más reciente de las opacidades OPAL (Iglesias \& Rogers, 1996) e incluyen tablas correpondientes a diferentes metalicidades, para permitir la evaluación de la opacidad en el caso de cualquier valor de la metalicidad. Como se verá más adelante, esto es de fundamental importancia en el estudio de las enanas blancas ya que la difusión microscópica produce en esos objetos gradientes de metalicidad en el interior estelar y las opacidades deben tener en cuenta esta variación dada su fuerte dependencia con la abundancia de metales. En el caso de bajas temperaturas las opacidades radiativas están complementadas con las opacidades moleculares de Alexander \& Ferguson (1994). Las opacidades conductivas en los regímenes de baja y alta densidad están tomadas de Hubbard \& Lampe (1969) y Itoh et al. (1983) respectivamente.

Las especies nucleares consideradas son $\mathrm{H}, \mathrm{D},{ }^{3} \mathrm{He},{ }^{4} \mathrm{He},{ }^{7} \mathrm{Li},{ }^{7} \mathrm{Be},{ }^{12} \mathrm{C},{ }^{13} \mathrm{C},{ }^{14} \mathrm{~N},{ }^{15} \mathrm{~N},{ }^{16} \mathrm{O}$, ${ }^{17} \mathrm{O},{ }^{18} \mathrm{O},{ }^{19} \mathrm{~F}$ y ${ }^{20} \mathrm{Ne}$ a menos que se indique lo contrario. La red de reacciones nucleares es la apropiada para seguir la evolución estelar durante las fases de combustión de hidrógeno y helio e incluye 30 reacciones nucleares. Para el primer caso se incluyen las reacciones nucleares de las tres cadenas del proceso protón-protón y el tri-ciclo CNOF. En el caso de la combustión del helio se incluyen las reacciones fundamentales que determinan la tasa de liberación de energía durante estas etapas. Las tasas de las reacciones nucleares están tomadas en su mayoría de la compilación de Caughlan \& Fowler (1988), excepto en el caso en que determinaciones más recientes difieren de manera apreciable. En particular es importante mencionar la reacción ${ }^{12} \mathrm{C}\left({ }^{4} \mathrm{He}, \gamma\right){ }^{16} \mathrm{O}$, que está tomada de Angulo C. et al. (1999). 


\section{Capítulo 3}

\section{Enanas blancas de baja masa I. Progenitores con metalicidad solar}

En este capítulo se presenta una serie de cálculos evolutivos de enanas blancas con núcleos de helio (HeWD) que cubren el rango de masas esperado para estos objetos. El objetivo principal se centra en estudiar los efectos de la difusión de elementos en la evolución de estos objetos. Para este fin se han hecho dos conjuntos de modelos, uno incluyendo la difusión y otro en el cual sus efectos son despreciados. Esto permite desentrañar con claridad la influencia de la difusión microscópica en la evolución de las HeWDs. Los modelos iniciales son obtenidos a partir de un modelo de $1 \mathrm{M}_{\odot}$ de metalicidad solar sometido a pérdida de masa para simular la evolución binaria que da origen a las HeWDs y permite obtener una cantidad fundamental en la evolución de estos objetos, el espesor de la envoltura de hidrógeno, de manera consistente.

Los resultados presentados en este capítulo han sido publicados en los artículos Althaus, Serenelli \& Benvenuto (2001a,b,c).

\subsection{Introducción}

En los últimos años, enanas blancas de baja masa con núcleos de helio (HeWDs) han sido detectadas en numerosos sistemas binarios. La predicción teórica de que las HeWDs serían el resultado de episodios de transferencia de masa en sistemas binarios cercanos (Kippenhahn, Kohl \& Weigert, 1967; Kippenhahn, Thomas \& Weigert, 1968; Webbink, 1975) ha sido corroborada observacionalmente por primera vez por Marsh (1995) y Marsh, Dhillon \& Duck (1995). Desde entonces, estos objetos han sido observados en distintos sistemas binarios conteniendo generalmente otra enana blanca (WD) o una estrella de neutrones (ver p.ej. Lundgren et al., 1996; Moran, Marsh \& Bragaglia, 1997; Orosz et al., 1999; Maxted et al., 2000). También es importante destacar que las HeWDs han comenzado a ser detecatadas en cúmulos globulares (Landsman et al., 1997; Edmonds et al., 1999; Taylor et al., 2001; Edmonds et al., 2001).

Una configuración importante en la cual pueden encontrarse HeWDs es en sistemas binarios en compañía de púlsares de milisegundo. Estos sistemas binarios permiten obtener valiosa información sobre las teorías tanto de evolución de enanas blancas como de púlsares de milisegundo. Si se supone que los púlsares de milisegundo son púlsares que han sido acelerados por la acreción de materia durante la fase de pérdida de masa que llevó a la formación de la WD, entonces la edad de enfriamiento de la WD y la edad del púlsar obtenida a partir de su spin-down deben ser coincidentes. Por lo tanto, en estos casos pueden obtenerse dos estima- 
ciones independientes de la edad del sistema y de esta manera puede revisarse la validez de las teorías. Dentro de este tipos de sistemas, quizás el mejor estudiado es el PSR J1012+5307. Los parámetros atmosféricos de la enana blanca de este sistema han sido determinados por van Kerkwijk, Bergeron \& Kulkarni S. R. (1996) y Callanan, Garnavich, Koester (1998). La edad de spin-down del púlsar es aproximadamente 7 Gyr (Lorimer et al., 1995) y puede ser comparada con las predicciones teóricas del enfriamiento de su WD compañera.

Respecto a los modelos teóricos de enfriamiento de HeWDs, Sarna, Ergma \& Antipova (2000) han presentado cálculos muy detallados de la evolución binaria que lleva a la formación de HeWDs, en particular con masas finales menores a $0.25 \mathrm{M}_{\odot}$. En estos cálculos ellos encuentran que luego de la etapa de transferencia de masa, y cuando el progenitor de HeWD se contrae dentro de su lóbulo de Roche, los núcleos de helio están cubiertos por una envoltura de hidrógeno masiva de $0.01-0.06 \mathrm{M}_{\odot} \mathrm{y}$ una abundancia superficial de hidrógeno $X_{\mathrm{H}}=0.35$ 0.50. Estas envolturas masivas también han sido encontradas por Driebe et al. (1998), quienes han seguido la evolución de una estrella de $1 \mathrm{M}_{\odot}$ desde la secuencia principal de edad cero (ZAMS) hasta la rama de las gigantes rojas (RGB). En diferentes posiciones a lo largo de la RGB los modelos son sometidos a tasas de pérdida de masa apropiadas para simular la evolución binaria hasta que la estrella evoluciona contrayéndose y alejándose de la RGB, dando lugar a HeWDs. Driebe et al. (1998) han seguido la evolución de sus modelos a lo largo de la curva de enfriamiento hasta luminosidades estelares muy bajas y han derivado relaciones masa-radio para analizar los recientes datos observacionales. Estos cálculos muestran que la ocurrencia de flashes termonucleares está restringida a un cierto rango de masas de las HeWDs entre $0.2-0.3 \mathrm{M}_{\odot}$ (ver también Webbink, 1975; Alberts et al., 1996). Estos modelos también predicen que, a consecuencia de las envolturas de hidrógeno masivas, la combustión nuclear del shell de hidrógeno es la fuente de energía dominante de la estrella aún para valores de la temperatura efectiva bien por debajo de 10000K. Esto conduce a edades de enfriamiento altas para las HeWDs, aún cuando sufran flashes termonucleares. En particular, estos modelos evolutivos predicen para la HeWD compañera del PSR J1012+5307 una edad de unos 7 Gyr, en buen acuerdo con la edad de spin-down del púlsar.

Sin embargo, recientes estudios observacionales han arrojado dudas sobre el espesor de las envolturas de hidrógeno en las HeWDs que predicen los cálculos evolutivos mencionados. En un trabajo reciente, van Kerkwijk et al. (2000) han detectado ópticamente la HeWD compañera del púlsar de milisegundo PSR B1855+09 y determinaron que su temperatura efectiva es 4800土 $800 \mathrm{~K}$. Además, la masa de esta HeWD ha sido determinada por medio del retraso de Shapiro de la señal del púlsar y es $0.258_{-0.016}^{+0.028} \mathrm{M}_{\odot}$ (Kaspi, Taylor \& Ryba, 1994). Según los modelos de Driebe et al. (1998), la edad de enfriamiento para esta HeWD es de $\tau_{W D} \approx 10 \mathrm{Gyr}$, sin embargo la edad de spin-down del púlsar es $\tau_{S D} \approx 5$ Gyr. Otros casos interesantes son los de PSR J0034-0534 y PSR J1713+0747, que tienen como compañeras enanas blancas frías (Hansen \& Phinney, 1998b) para las cuales las edades inferidas a partir de los modelos de Driebe et al. (1998) están bien por encima de los 10 Gyr. Como ha sido sugerido por van Kerkwijk et al. (2000), estos modelos pueden estar sobreestimando las edades de enfriamiento de las HeWDs.

La masa de la envoltura de hidrógeno resulta ser la cantidad fundamental de la cual depende la edad de enfriamiento de una HeWD. Cuando la envoltura de hidrógeno es delgada la temperatura en su base no es lo suficientemente alta como para que las reacciones nucleares, en particular la fusión del hidrógeno, contribuyan apreciablemente como fuente de energía de la estrella. En este caso la HeWD simplemente se enfría, siendo el calor almacenado por los iones en su interior la única fuente de energía relevante, y dando lugar a un enfriamiento rápido de la HeWD. Por el contrario, si la envoltura de hidrógeno es masiva durante el enfriamiento de 
la HeWD, entonces la fusión del hidrógeno en el shell será una fuente de energía importante para la estrella, que tendrá entonces un enfriamiento lento, es decir que se tendrán edades de enfriamiento grandes. La evolución binaria indica que las HeWD se forman con envolturas de hidrógeno masivas. Existen sin embargo varios mecanismos por los cuales estas envolturas pueden ser reducidas. Por ejemplo, si una HeWD sufre un flash termonuclear sus dimensiones pueden alcanzar otra vez las de una gigante roja y eventualmente llenar nuevamente su lóbulo de Roche, dando lugar a una nueva, pero breve, etapa de transferencia de masa. Esta posibilidad ha sido explorada por Iben \& Tutukov (1986), quienes siguieron la evolución de un modelo de HeWD de $0.296 \mathrm{M}_{\odot}$ remanente de un sistema binario. Estos autores encuentran que su modelo experimenta dos flashes termonucleares y que como consecuencia de éstos el modelo se expande llenando nuevamente su lóbulo de Roche y dando así lugar a una nueva fase de pérdida de masa. Durante esta fase la envoltura de hidrógeno es reducida considerablemente, dando lugar a edades de enfriamiento cortas en etapas avanzadas de la evolución. En este sentido, la existencia de flashes termonucleares en el shell de hidrógeno puede jugar un papel preponderante en la determinación del espesor de la envoltura de hidrógeno y, por lo tanto, en el establecimiento de las edades de enfriamiento reales de las HeWDs. Otra posibilidad respecto a la reducción de la envoltura de hidrógeno ha sido propuesta por Sarna, Ergma \& Antipova (2000), quienes argumentan que una pre-HeWD de baja masa puede perder una considerable parte de su envoltura debido a la irradiación del púlsar.

En los cálculos que se presentan en esta tesis, el hincapié está hecho en estudiar el rol que juega la difusión de los elementos en la evolución de las HeWDs. En particular, la ocurrencia de los flashes del shell de hidrógeno está ligada directamente a la difusión y, como se mostrará, esto tiene influencia directa en la reducción de la envoltura de hidrógeno. La difusión química es responsable de que la cola interna del perfil de hidrógeno en la base de la envoltura se difunda hacia adentro, a regiones de temperaturas más elevadas (Iben \& MacDonald, 1985; Althaus \& Benvenuto, 2000). En el caso de un modelo de $\mathrm{HeWD}$ de $0.296 \mathrm{M}_{\odot}$, esta característica ha sido señalada como posible disparador de un flash termonuclear (Iben \& Tutukov, 1986).

Los procesos de difusión han sido considerados por numerosos investigadores desde el temprano trabajo de Schatzman (1958) sobre el rol de la difusión en la evolución de la composición química superficial de las WDs. Desde entonces, diferentes estudios han mostrado que la sedimentación gravitatoria produce que los elementos más pesados que los constituyentes atmosféricos promedios se hundan debajo de la fotosfera en escalas de tiempo mucho más cortas que las evolutivas, dando así una explicación de la pureza de la mayor parte de las atmósferas de las WDs (Fontaine \& Michaud, 1979; Alcock \& Illarionov, 1980; Muchmore, 1984; Iben \& MacDonald, 1985; Paquette et al., 1986b; Dupuis et al., 1992, entre otros). En el contexto de la evolución de HeWD, Iben \& Tutukov (1986) han encontrado que la difusión lleva hidrógeno hacia el interior estelar a través de capas calientes de la estrella, suficientes para encender el hidrógeno de una manera inestable, produciendo un flash termonuclear. Recientemente, Althaus \& Benvenuto (2000) han explorado los efectos de la difusión en la relación masa-radio de las HeWDs.

\subsection{Ecuaciones de difusión}

El código de evolución estelar utilizado en estos cálculos es el CEELP, cuyas características generales y física constitutiva incluida han sido descriptos en el Cap. 2. No volveremos aquí sobre ellos. Sin embargo, es importante destacar la inclusión en el CEELP de la difusión 
microscópica, que como se verá más adelante resulta de importancia fundamental en la evolución de las HeWDs.

El tratamiento de las ecuaciones de difusión sigue los lineamientos presentados por Iben \& MacDonald (1985), aunque incluye también la difusión térmica, que no es tenida en cuenta por esos autores. La difusión microscópica de las especies nucleares incorporada en el CEELP incluye la sedimentación gravitatoria y las difusiones química y térmica. Bajo la influencia de la gravedad, presiones parciales, gradientes térmicos y campos eléctricos inducidos (los campos magnéticos y la rotación estelar son despreciados) las velocidades de difusión en un gas multicomponente (Burgers, 1969) satisfacen el conjunto de ecuaciones de difusión $(N-1$ ecuaciones lineales independientes)

$$
\frac{d p_{i}}{d r}-\frac{\rho_{i}}{\rho} \frac{d p}{d r}-n_{i} Z_{i} e E=\sum_{j \neq i}^{N} K_{i j}\left(w_{j}-w_{i}\right)+\sum_{j \neq i}^{N} K_{i j} z_{i j} \frac{m_{j} r_{i}-m_{i} r_{j}}{m_{i}+m_{j}}
$$

y las $N$ ecuaciones de flujo del calor

$$
\begin{aligned}
\frac{5}{2} n_{i} k_{B} \nabla T= & -\frac{5}{2} \sum_{j \neq i}^{N} K_{i j} z_{i j} \frac{m_{j}}{m_{i}+m_{j}}\left(w_{j}-w_{i}\right)-\frac{2}{5} K_{i i} z_{i i}^{\prime \prime} r_{i} \\
& -\sum_{j \neq i}^{N} \frac{K_{i j}}{\left(m_{i}+m_{j}\right)^{2}}\left(3 m_{i}^{2}+m_{j}^{2} z_{i j}^{\prime}+0.8 m_{i} m_{j} z_{i j}^{\prime \prime}\right) r_{i} \\
& +\sum_{j \neq i}^{N} \frac{K_{i j} m_{i} m_{j}}{\left(m_{i}+m_{j}\right)^{2}}\left(3+z_{i j}^{\prime}-0.8 z_{i j}^{\prime \prime}\right) r_{j} .
\end{aligned}
$$

Aquí, $p_{i}, \rho_{i}, n_{i}, Z_{i}$ and $m_{i}$ son, respectivamente, la presión parcial, la densidad de masa, la densidad número, la carga media y la masa para las especies $i$ ( $N$ es el número total de especies más el electrón). $T, \nabla T$ y $k_{B}$ son la temperatura, el gradiente de temperatura y la constante de Boltzmann respectivamente. Las variables desconocidas son las velocidades de difusión con respecto al centro de masa, $w_{i}$, y los flujos residuales de calor $r_{i}$ (para iones y electrones). También el campo eléctrico $E$ debe ser determinado. Los coeficientes de resistencia $K_{i j}, z_{i j}$, $z_{i j}^{\prime}$ y $z_{i j}^{\prime \prime}$ están tomados de Paquette et al. (1986a) y las cargas iónicas promedio están tratadas siguiendo un modelo aproximado de ionización por presión dado por Paquette et al. (1986b).

Para completar el conjunto de ecuaciones, usamos las condiciones de flujo de masa nulo con respecto al centro de masa

$$
\sum_{i} A_{i} n_{i} w_{i}=0
$$

y corriente eléctrica nula

$$
\sum_{i} Z_{i} n_{i} w_{i}=0
$$

En términos del gradiente en la densidad número, podemos transformar la ecuación (3.1) en

$$
\begin{array}{r}
\frac{1}{n_{i}}\left[\sum_{j \neq i}^{N} K_{i j}\left(w_{i}-w_{j}\right)+\right. \\
\left.\sum_{j \neq i}^{N} K_{i j} z_{i j} \frac{m_{i} r_{j}-m_{j} r_{i}}{m_{i}+m_{j}}\right] \\
-Z_{i} e E=\alpha_{i}-k_{B} T \frac{d \ln n_{i}}{d r}
\end{array}
$$


donde

$$
\alpha_{i}=-A_{i} m_{H} g-k_{B} T \frac{d \ln T}{d r}
$$

y $A_{i}, m_{H}$ y $g$ son el número de masa atómica, la masa del átomo de hidrógeno y la gravedad. Las incógnitas $w_{i}, r_{i}$ y $E$ pueden escribirse en términos de los gradientes de las densidades de los iones (similarmente para $r_{i}$ and $E$ )

$$
w_{i}=w_{i}^{g t}-\sum_{i o n s(j)} \sigma_{i j} \frac{d \ln n_{j}}{d r}
$$

donde $w_{i}^{g t}$ es la componente de la velocidad debida a la sedimentación gravitatoria y la difusión térmica. La suma (3.7) debe ser efectuada sólo sobre los iones. Las ecuaciones (3.2-3.5) permiten hallar las componentes $w_{i}^{g t}$ y $\sigma_{i j}$ mediante inversiones matriciales, que en el CEELP son resueltas mediante una descomposición LU (Press et al., 1986).

Las ecuaciones de difusión presentadas permiten conocer la evolución de la distribución de elementos químicos en el interior de la estrella resolviendo la ecuación de continuidad. En los presentes cálculos se ha considerado la evolución de los isótopos ${ }^{1} \mathrm{H},{ }^{3} \mathrm{He},{ }^{4} \mathrm{He},{ }^{12} \mathrm{C},{ }^{14} \mathrm{~N}$ y ${ }^{16}$ O. Para poder calcular la dependencia de la estructura de los modelos de HeWDs con la variación de las abundancias de manera auto-consistente, el conjunto de ecuaciones de difusión ha sido acoplado al CEELP. Durante el cálculo de los modelos, en cada paso evolutivo las ecuaciones de difusión son resueltas y luego de que los cambios en las abundancias por efectos de la difusión son obtenidos, las abundancias resultantes son evolucionadas de acuerdo a los cambios producidos por las reacciones nucleares y la mezcla convectiva (en el caso en que esté presente).

Finalmente, un punto importante a tener en cuenta es que los cambios de composición química inducidos por la difusión producen en la estrella gradientes de metalicidad. La sedimentación gravitatoria, como se mostrará en las secciones siguientes, rápidamente produce el hundimiento de los metales de las capas más externas de la HeWD (y en escalas temporales un poco más largas del helio). El cálculo de la opacidad debe tener en cuenta la variación de la metalicidad, por lo que se han incluido en el cálculo las tablas de opacidades radiativas de OPAL (Iglesias \& Rogers, 1996) para diferentes metalicidades, que permiten una interpolación para cualquier metalicidad. En particular, se ha tomado la metalicidad de la materia estelar igual al doble de la suma de las abundancias de los núcleos representativos del ciclo CNO $\left({ }^{12} \mathrm{C}\right.$, ${ }^{14} \mathrm{~N}$ y $\left.{ }^{16} \mathrm{O}\right)$.

\subsection{Modelos iniciales}

Como se mencionó en la sección 3.1, el espesor de la envoltura de hidrógeno resulta de fundamental importancia para determinar las escalas de tiempo en las cuales las HeWDs se enfrían. Por este motivo es necesario obtener modelos iniciales de HeWD lo más realistas posibles. Para este fin, hemos considerado un modelo estelar de $1 \mathrm{M}_{\odot}$ inicial y metalicidad solar, en la ZAMS. La evolución de este modelo se ha seguido detalladamente durante su evolución en la secuencia principal hasta su evolución en la RGB. En diferentes posiciones en la RGB, el modelo se ha sometido a diferentes tasas de pérdida de masa apropiadas para simular la evolución binaria hasta que la envoltura de hidrógeno se vuelve lo suficientemente delgada como para que no pueda mantenerse extendida. En ese momento, la envoltura se contrae y la pérdida de masa se 
detiene, dando lugar a una pre-HeWD, que se desplaza rápidamente hacia el azul en el diagrama Hertzsprung-Russell (HRD) mientras se contrae. De esta manera, se han obtenido modelos iniciales de HeWD con masas de 0.406, 0.360, 0.327, 0.292, 0.242, 0.196, 0.169 y $0.161 \mathrm{M}_{\odot}$, que abarcan el rango de masas esperados para estos objetos (recordemos que la masa máxima que puede tener un núcleo de helio sin que éste comience a fusionarse es $\sim 0.45 \mathrm{M}_{\odot}$ ). En la tabla 3.1 se presentan las principales características de los modelos iniciales de HeWD obtenidos. Además de su masa, se presentan la masa de la envoltura de hidrógeno y la abundancia superficial de hidrógeno en el punto de mayor temperatura efectiva alcanzada (codo) antes de comenzar a enfriarse. Es interesante destacar que las características de las envolturas resultantes muestran un buen acuerdo con los resultados de Driebe et al. (1998), en particular para los modelos más masivos. Para los modelos menos masivos las envolturas resultan un poco más masivas que las de Driebe et al. (1998). Es interesante destacar que Sarna, Ergma \& Antipova (2000) obtienen envolturas muy masivas en base a cálculos binarios. Si bien el método empleado en nuestros cálculos es una simplificación de la situación real, donde se espera que ocurra una fase de envoltura común, la evolución subsiguiente de la HeWD no depende de los detalles de cómo la envoltura fue perdida. En particular, la estructura termo-mecánica de los modelos es consistente con las predicciones de cálculos de evolución binaria (ver Driebe et al., 1998).

Table 3.1: Masa estelar, masa de la envoltura y abundancia superficial de hidrógeno $X_{H}$ en el punto de máxima temperatura efectiva.

\begin{tabular}{ccc}
\hline$M / \mathrm{M}_{\odot}$ & $M_{e n v} / \mathrm{M}_{\odot}$ & $X_{H}$ \\
\hline 0.406 & $7.3 \times 10^{-4}$ & 0.701 \\
0.360 & $1.1 \times 10^{-3}$ & 0.701 \\
0.327 & $1.4 \times 10^{-3}$ & 0.701 \\
0.292 & $2.0 \times 10^{-3}$ & 0.701 \\
0.242 & $3.7 \times 10^{-3}$ & 0.694 \\
0.196 & $6.7 \times 10^{-3}$ & 0.504 \\
0.169 & $1.0 \times 10^{-2}$ & 0.423 \\
\hline
\end{tabular}

Estos valores corresponden a las secuencias en que la difusión ha sido despreciada

\subsection{Resultados}

En esta sección se presentan los resultados de los cálculos evolutivos correspondientes a los dos conjuntos de modelos calculados, es decir, con y sin difusión. En 3.4.1 se discuten ampliamente las diferencias evolutivas causadas por la inclusión de la difusión microscópica en los cálculos evolutivos de las HeWDs. Los flashes termonucleares que la difusión induce en la mayor parte de los modelos de HeWDs afectan notablemente la evolución de estos objetos. Dada la importancia de los flashes termonucleares en la vida de las HeWD, en 3.4.2 se describe en detalle la evolución y estructura de un modelo de HeWD durante esta etapa.

\subsubsection{Comparación entre modelos con y sin difusión}

Una vez que los modelos pre-HeWD se contraen, alejándose de la RGB, la pérdida de masa fue suprimida de modo que la evolución subsiguiente es a masa constante. La evolución de 


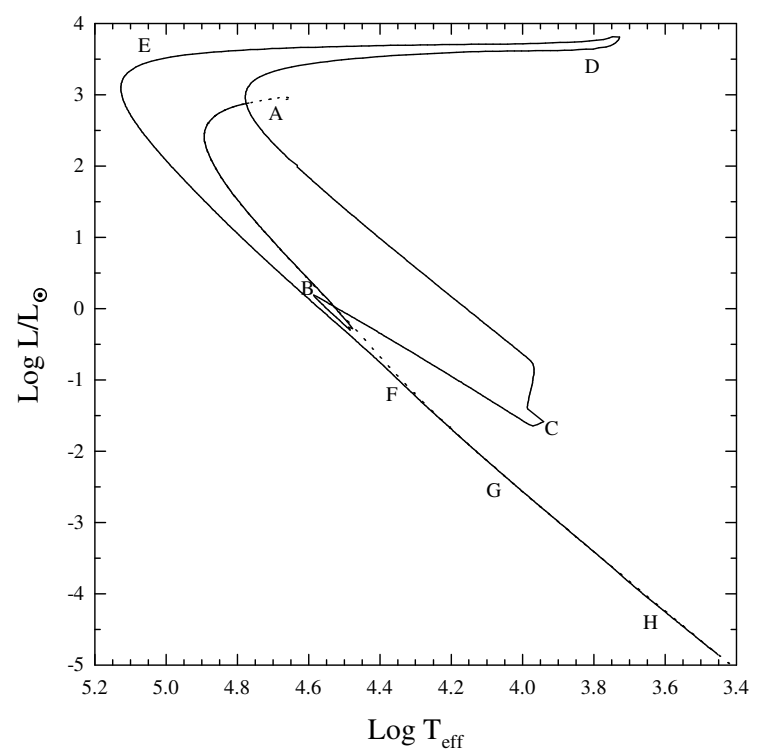

Figura 3.1: Tracks evolutivos en el diagrama HR teórico para el modelo de HeWD de $0.406 \mathrm{M}_{\odot}$. La secuencia que incluye la difusión se muestra en línea sólida mientras que el modelo estándar (sin difusión) se muestra en línea punteada. Con letras mayúsculas se indican algunos estados evolutivos particulares cuyas características se presentan en la tabla 3.2. Notar el loop que describe el modelo con difusión como resultado del flash termonuclear del shell de hidrógeno, inducido por la difusión química, que fuerza a la estrella a alcanzar nuevamente dimensiones de gigante roja (punto D). El modelo sin difusión no sufre ningún flash nuclear.

estos modelos fue seguida hasta luminosidades muy bajas $\left(\log \mathrm{L} / \mathrm{L}_{\odot}=-5\right)$. Como ya se mencionó, el objetivo principal de esta investigación es determinar la influencia de la difusión en la evolución de las HeWDs, por lo que se han realizado dos conjuntos de cálculos evolutivos, uno de los cuales incluye los efectos de la difusión y otro en el cual éstos son despreciados. En ambos casos, los modelos considerados tienen 0.406, 0.360, 0.327, 0.292, 0.242, 0.196, 0.169 y $0.161 \mathrm{M}_{\odot}$ de manera de cubrir el rango de masas esperado para las HeWDs. Esto permitirá determinar claramente los efectos de la difusión en la evolución de estos objetos. Con respecto a los modelos que no incluyen difusión (que denominaremos modelos estándar), y que son tomados en este trabajo sólo como punto de referencia, es importante destacar que los resultados que de ellos se derivan están en excelente acuerdo con los modelos de Driebe et al. (1998), quienes no tienen en cuenta la difusión.

En las figuras 3.1 a 3.4 se muestra la evolución en el HRD de los modelos de HeWD de 0.406, 0.242, 0.196 y $0.169 \mathrm{M}_{\odot}$ incluyendo (líneas sólidas) y despreciando (líneas punteadas) los efectos de la difusión. Algunas características de los modelos con difusión, correspondientes a estados evolutivos denotados con letras en estas figuras se presentan en la tabla 3.2, donde se listan la masa estelar, la luminosidad superficial, la temperatura efectiva, la edad, la gravedad superficial, la abundancia superficial de hidrógeno y la masa de la envoltura de hidrógeno. La característica más importante ilustrada por estas figuras es que la difusión juega un papel fundamental en la inducción de la ocurrencia de flashes termonucleares que, como veremos, son críticos con respecto a la evolución subsiguiente de estos objetos aún durante su etapa final de enfriamiento. Durante los episodios de flash, la estrella evoluciona rápidamente y en la mayor parte de los casos sufre una expansión que la lleva nuevamente a la región de la 


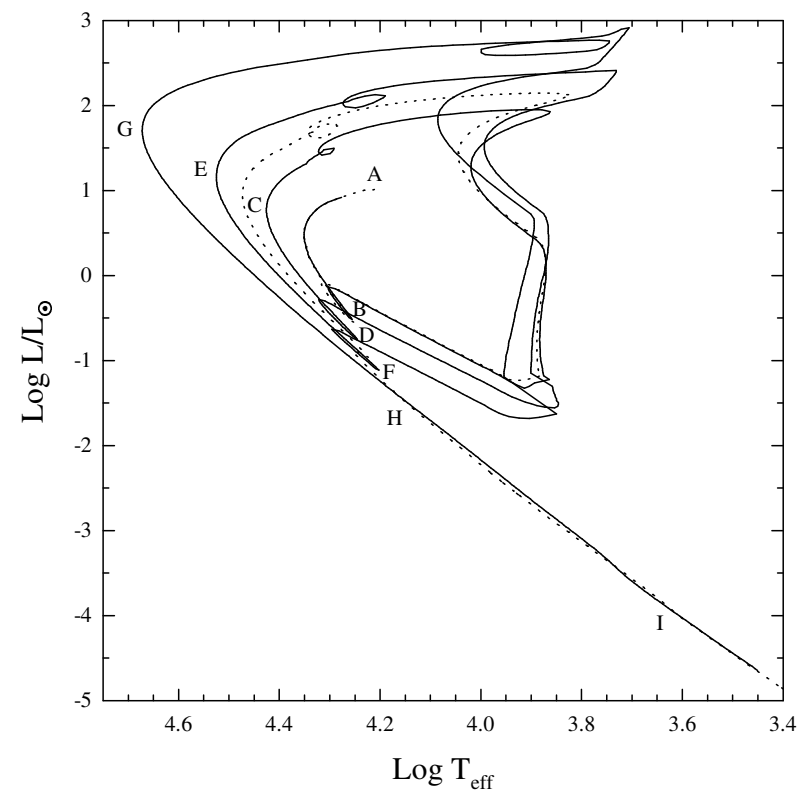

Figura 3.2: Igual que la figura 3.1, pero para el modelo de $0.242 \mathrm{M}_{\odot}$. En este caso la secuencia con difusión sufre tres flashes termonucleares, sucesivamente más intensos. Los pequeños loops en el trayecto horizontal se deben a aumentos temporales en la liberación de energía nuclear debido a la compresión de la base de la envoltura de hidrógeno. La secuencia estándar sufre sólo un flash termonuclear, que no resulta suficiente para reducir apreciablemente su envoltura de hidrógeno, lo que resulta en una evolución lenta a lo largo de la curva de enfriamiento.

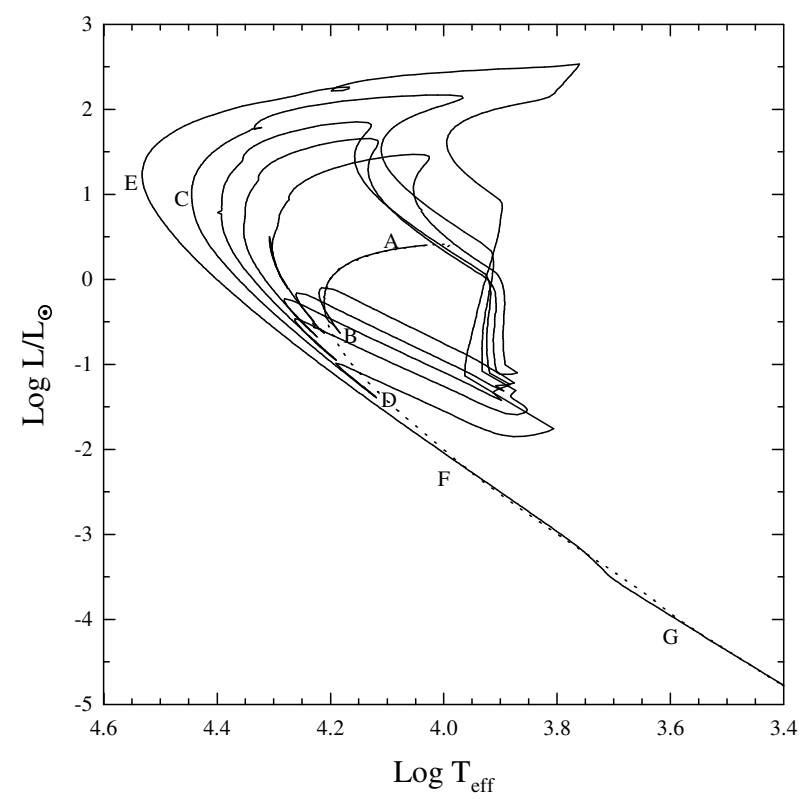

Figura 3.3: Igual que la figura 3.1, pero para el modelo de $0.196 \mathrm{M}_{\odot}$. El modelo con difusión sufre en este caso cinco flashes termonucleares, mientras que el modelo estándar no sufre ninguno. La evolución subsiguiente estará caracterizada por escalas de tiempo marcadamente diferentes en un caso y en otro. 
Table 3.2: Algunos estados evolutivos seleccionados para los modelos de HeWD de 0.406, 0.242, 0.196 y $0.169 \mathrm{M}_{\odot}$ considerando los efectos de la difusión.

\begin{tabular}{|c|c|c|c|c|c|c|c|}
\hline$M_{*} / \mathrm{M}_{\odot}$ & $\log \mathrm{L} / \mathrm{L}_{\odot}$ & $\log \mathrm{T}_{\mathrm{eff}}$ & Age $\left(10^{6} \mathrm{yr}\right)$ & $\log (g)$ & $\log \left(L_{n u c} / \mathrm{L}_{\odot}\right)$ & $X_{H}$ & $\log \left(M_{H} / \mathrm{M}_{*}\right)$ \\
\hline $0.406(\mathrm{~A})$ & 2.9465 & 4.7001 & 0.050 & 4.8559 & 2.9426 & 0.7007 & -2.764 \\
\hline$"(\mathrm{~B})$ & 0.4136 & 4.6003 & 0.208 & 6.9897 & -0.6597 & 0.9690 & -2.990 \\
\hline$"(\mathrm{C})$ & -1.6085 & 3.950 & 13.47880 & 6.4105 & 6.2738 & 0.9999 & -3.112 \\
\hline$"(\mathrm{D})$ & 3.6399 & 3.800 & 13.47882 & 0.5621 & 3.4811 & 0.3217 & -3.118 \\
\hline$"(\mathrm{E})$ & 3.4490 & 5.0547 & 13.48295 & 5.7717 & 3.4427 & 0.3217 & -3.454 \\
\hline$"(\mathrm{~F})$ & -0.7533 & 4.400 & 16.782 & 7.3555 & -2.4647 & 0.9997 & -3.599 \\
\hline$"(\mathrm{G})$ & -2.1305 & 4.100 & 351.53 & 7.5339 & -3.7283 & 0.9999 & -3.628 \\
\hline$"(\mathrm{H})$ & -4.0503 & 3.650 & 8334.90 & 7.6527 & -7.5068 & 1.0000 & -3.634 \\
\hline$\overline{0.242(\mathrm{~A})}$ & 1.0199 & 4.2099 & 17.00 & 4.5962 & 1.0193 & 0.6936 & -1.892 \\
\hline$"(\mathrm{~B})$ & -0.5299 & 4.2538 & 33.057 & 6.3219 & -0.6130 & 0.9999 & -2.035 \\
\hline$"(\mathrm{C})$ & 0.7534 & 4.4258 & 40.209 & 5.7263 & 0.7457 & 0.9996 & -2.155 \\
\hline$"(\mathrm{D})$ & -0.7527 & 4.2454 & 45.827 & 6.5107 & -0.8618 & 1.0000 & -2.168 \\
\hline$"(\mathrm{E})$ & 1.0765 & 4.5238 & 54.70 & 5.7951 & 1.0684 & 0.9820 & -2.347 \\
\hline$"(\mathrm{~F})$ & -1.1160 & 4.2025 & 71.695 & 6.7027 & -1.2617 & 0.9997 & -2.363 \\
\hline$"(G)$ & 1.7033 & 4.6722 & 95.974 & 5.7620 & 1.6940 & 0.4798 & -2.676 \\
\hline$"(\mathrm{H})$ & -1.3322 & 4.1809 & 100.59 & 6.8326 & -1.9306 & 0.9997 & -2.725 \\
\hline$"(\mathrm{I})$ & -3.8149 & 3.6506 & 4222.8 & 7.1942 & -5.8365 & 0.9997 & -2.743 \\
\hline $0.196(\mathrm{~A})$ & 0.3393 & 4.1000 & 39.53 & 4.7470 & 0.3367 & 0.5042 & -1.679 \\
\hline$"(B)$ & -0.6347 & 4.1828 & 235.43 & 6.0509 & -0.6521 & 1.0000 & -1.910 \\
\hline$"(\mathrm{C})$ & 1.0140 & 4.4448 & 315.95 & 5.4503 & 1.0088 & 0.7750 & -2.311 \\
\hline$"(D)$ & -1.4105 & 4.1146 & 386.99 & 6.5538 & -1.4687 & 0.9997 & -2.376 \\
\hline$"(E)$ & 1.2206 & 4.5322 & 418.34 & 5.5932 & 1.2068 & 0.2387 & -2.645 \\
\hline$"(\mathrm{~F})$ & -2.0401 & 4.0004 & 495.09 & 6.7266 & -2.4071 & 0.9997 & -2.718 \\
\hline$"(G)$ & -3.9494 & 3.6011 & 4520.7 & 7.0388 & -6.9530 & 0.9403 & -2.727 \\
\hline $0.169(\mathrm{~A})$ & -0.7204 & 4.0425 & 480.75 & 5.5118 & -0.72463 & 0.9996 & -1.685 \\
\hline$"(B)$ & -2.0476 & 3.9250 & 4629.6 & 6.3691 & -2.05169 & 1.0000 & -1.875 \\
\hline$"(\mathrm{C})$ & -2.3589 & 3.8750 & 11211 & 6.4804 & -2.36304 & 1.0000 & -1.956 \\
\hline
\end{tabular}

Las edades se cuentan desde el final de la pérdida de masa (a $\mathrm{T}_{\text {eff }}=5000 \mathrm{~K}$ para los modelos de 0.406 y $0.242 \mathrm{M}_{\odot}, \mathrm{y} \mathrm{T}_{\text {eff }}=10000 \mathrm{~K}$ para los de 0.196 y $\left.0.169 \mathrm{M}_{\odot}\right)$. 


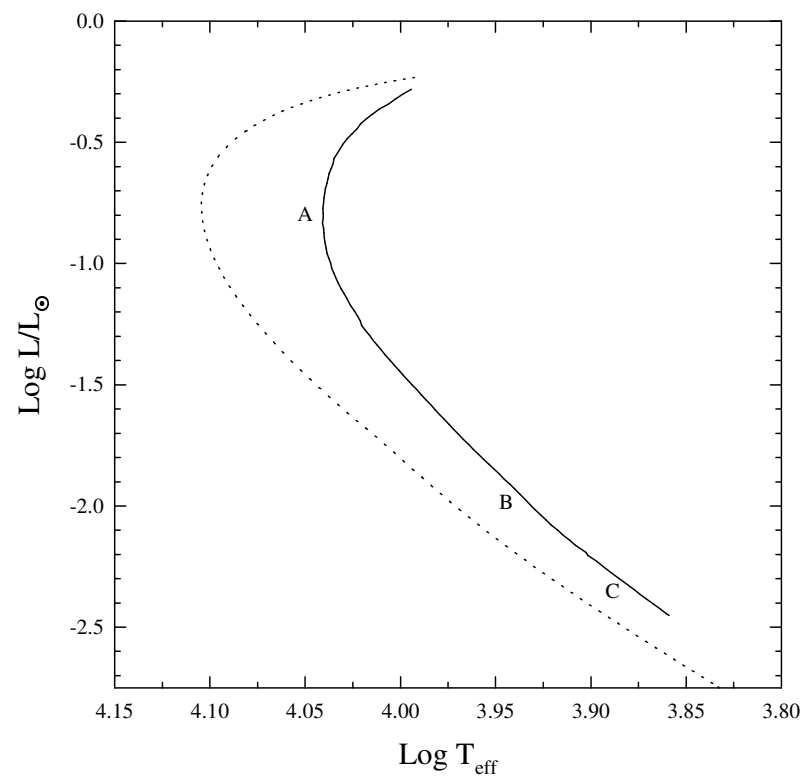

Figura 3.4: Igual que la figura 3.1, pero para el modelo de $0.169 \mathrm{M}_{\odot}$. A diferencia de los modelos más masivos, para esta masa, los modelos de HeWD no sufren flashes, aún en presencia de difusión. Esto tiene como consecuencia edades de enfriamiento similares en ambos casos. Sin embargo, en este caso la difusión da como resultado modelos marcadamente menos compactos como resultado del menor peso molecular promedio de las capas externas de la estrella.

RGB en el HRD. Cuando la difusión es considerada, todos nuestros modelos excepto los de 0.169 y $0.161 \mathrm{M}_{\odot}$ experimentan flashes termonucleares. Notar en cambio que si la difusión no es tenida en cuenta, sólo el modelo de $0.242 \mathrm{M}_{\odot}$ sufre una inestabilidad térmica, de acuerdo con las predicciones de Driebe et al. (1998), quienes encuentran que sólo sus modelos de 0.234 y $0.259 \mathrm{M}_{\odot}$ experimentan flashes termonucleares. Los modelos menos masivos, de 0.169 y $0.161 \mathrm{M}_{\odot}$ que no sufren flashes resultan mucho menos compactos cuando la difusión es tenida en cuenta, lo que afecta directamente la relación masa-radio de estos modelos.

Los efectos de la difusión en la distribución de elementos dentro de las estrellas se muestran en las figuras 3.5 a 3.7. En estas figuras las abundancias del ${ }^{1} \mathrm{H},{ }^{3} \mathrm{He},{ }^{4} \mathrm{He},{ }^{12} \mathrm{C},{ }^{14} \mathrm{~N}$ y ${ }^{16} \mathrm{O}$ son graficadas en función de la fracción de masa externa $q\left(q=1-M_{r} / M_{*}\right)$ para los modelos de 0.406, 0.242 y $0.169 \mathrm{M}_{\odot}$ en diferentes épocas de su evolución. En cada caso, los paneles (a) muestran la estratificación química inicial de los modelos, resultantes de la evolución pre-WD, antes de que la estrella alcance la rama de enfriamiento por primera vez. En el caso del modelo de $0.169 \mathrm{M}_{\odot}$ la difusión ya ha modificado la composición en las capas externas. Para los modelos menos masivos, las abundancias iniciales en las capas externas de la estrella difieren de las abundancias interestelares debido a que por efectos de la pérdida de masa han quedado expuestas regiones donde hubo procesamiento de los isótopos del CNO debido a la combustión del hidrógeno. En efecto, prácticamente todo el ${ }^{12} \mathrm{C}$ ha sido convertido a ${ }^{14} \mathrm{~N}$. El efecto que la difusión tiene en la distribución de elementos durante la evolución de los modelos es claramente notable en los modelos analizados en las figuras. Notar que la difusión rápidamente hace que el hidrógeno "flote" hacia la superficie, dando lugar a envolturas de hidrógeno puro. Al mismo tiempo, la cola interna del perfil de hidrógeno se difunde hacia el interior de la estrella por efectos de la difusión química (que actúa en contra del gradiente de composición), llevando 

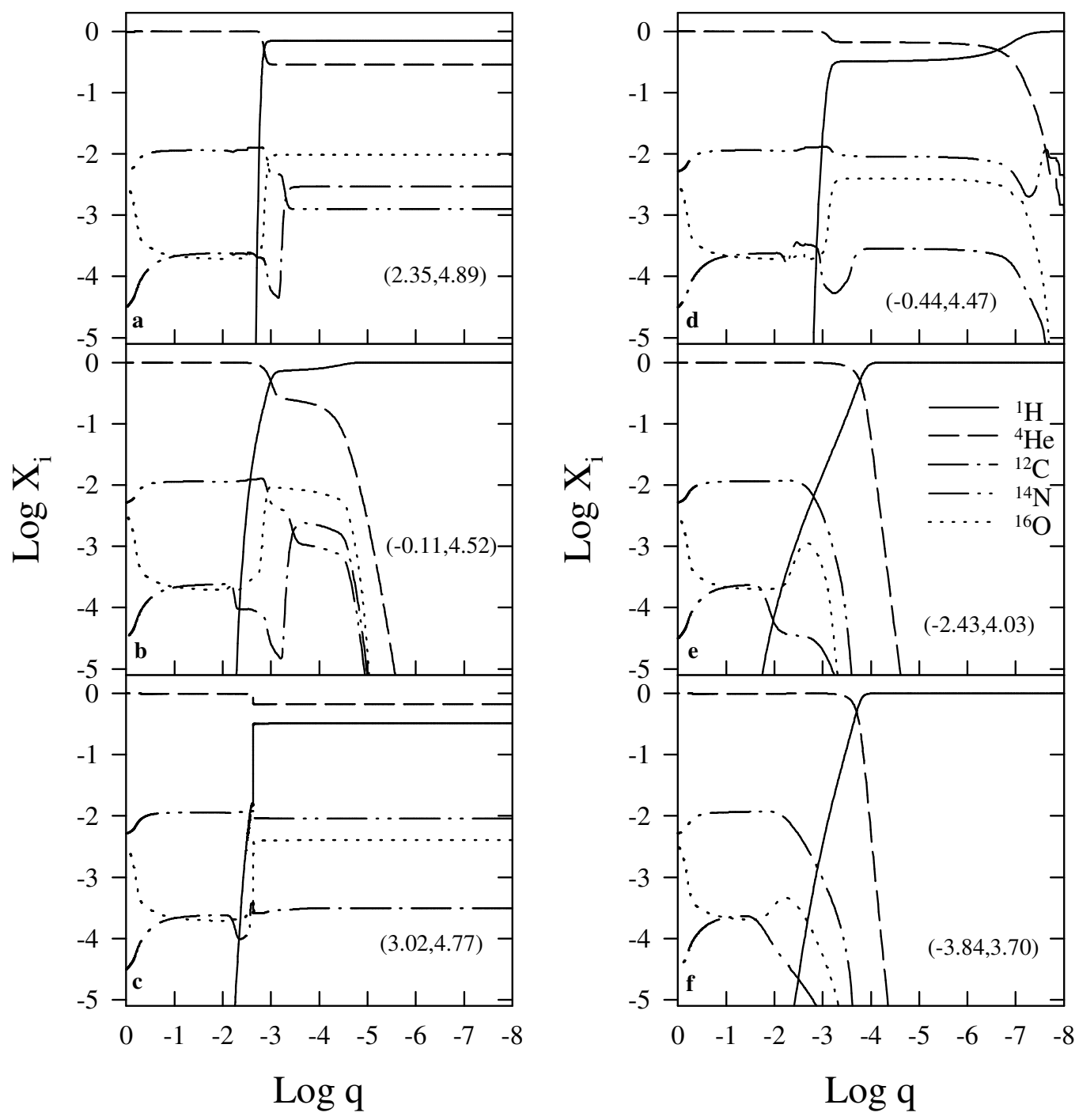

Figura 3.5: Abundancia en fracción de masa de ${ }^{1} \mathrm{H},{ }^{3} \mathrm{He},{ }^{4} \mathrm{He},{ }^{12} \mathrm{C},{ }^{14} \mathrm{~N}$ y ${ }^{16} \mathrm{O}$ contra la fracción de masa externa $q=1-M_{r} / M_{*}$ para el modelo de $0.406 \mathrm{M}_{\odot}$ correspondientes a estados evolutivos caracterizados por valores de $\log \mathrm{L} / \mathrm{L}_{\odot} \mathrm{y} \log \mathrm{T}_{\text {eff }}$ dados entre paréntesis. El panel (a) muestra la estratificación química inicial antes de que el modelo entre en la curva de enfriamiento por primera vez. El panel (b) corresponde al momento inmediatamente anterior de la ocurrencia del flash en el shell de hidrógeno y (c) muestra la estratificación inmediatamente antes del episodio de mezcla y antes de que la estrella vuelva a la zona de la RGB. Finalmente, los paneles (d), (e) y (f) ilustran la evolución de los perfiles químicos internos durante la rama de enfriamiento final. La difusión altera notablemente la estratificación química. Por un lado lleva a envolturas de hidrógeno puro en escalas de tiempo cortas debido a la sedimentación gravitatoria, que es favorecida por las altas gravedades de la WD. Por otro lado, la difusión química hace que la cola interna del perfil de hidrógeno penetre hacia zonas más internas de la estrella, y por lo tanto más calientes (paneles b y c). A valores altos de $\mathrm{T}_{\text {eff }}$, este efecto es el responsable de la ocurrencia del episodio de flash en el shell de hidrógeno. 


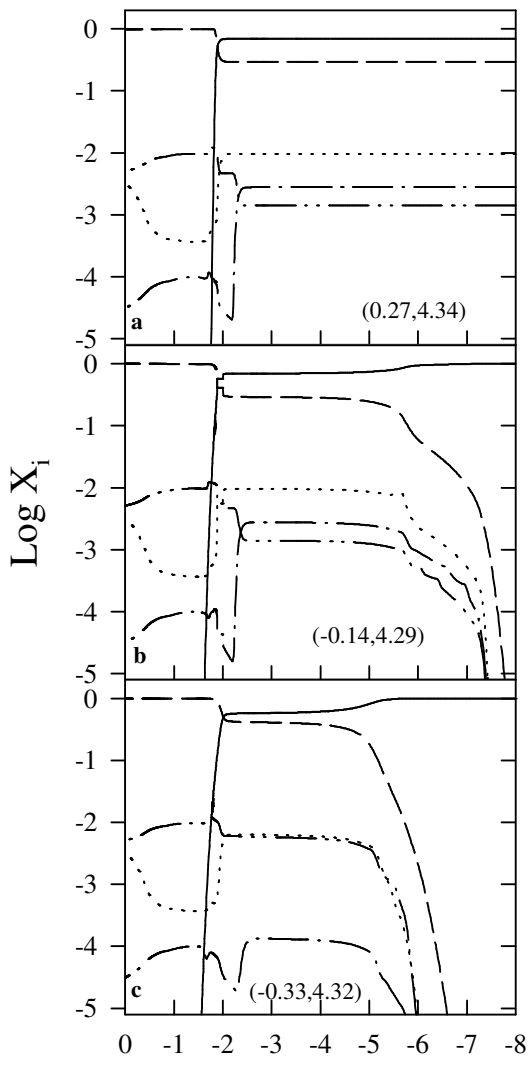

$\log \mathrm{q}$

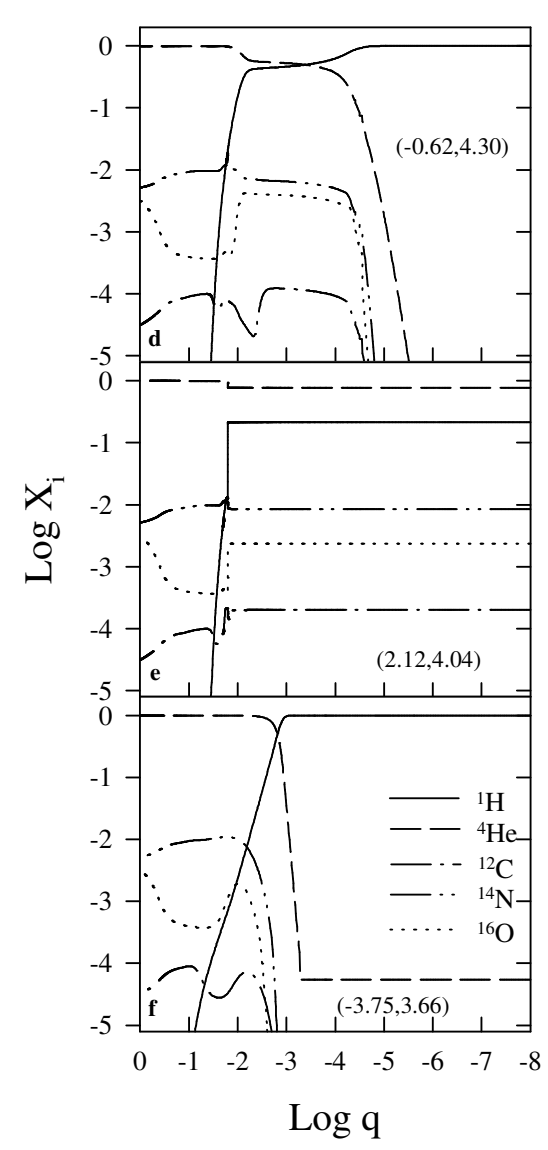

$\log \mathrm{q}$

Figura 3.6: Igual que figura 3.5 pero para el modelo de $0.242 \mathrm{M}_{\odot}$. Aquí los paneles (b), (c) y (d) correspondes a las fases evolutivas justo antes de la ocurrencia de cada episodio de flash (este modelo sufre tres de estos episodios). El panel (e) muestra la estratificación química después del episodio de mezcla inducido por el flash, antes de que la estrella vuelva a la RGB por última vez. Finalmente, el panel (f) corresponde a un estoda evolutivo avanzado donde hay un poco de helio presente en las capas externas como resultado de la mezcla convectiva. Ver texto para más detalles.

hidrógeno a capas más calientes. Eventualmente, el aumento en la liberación de energía nuclear produce una inestabilidad térmica (favorecida por la geometría del shell, ver Kippenhahn \& Weigert 1990) que dispara el flash termonuclear. Durante los episodios de flash, las regiones de la estrella ubicadas inmediatamente por encima de la región donde la liberación de energía nuclear es máxima se vuelven inestables frente a la convección. Esta zona convectiva se extiende paulatinamente hacia la superficie y cuando la alcanza las abundancias superficiales resultan drásticamente modificadas (paneles c y e de las figuras 3.5 y 3.6 respectivamente). Después de un flash, el modelo evoluciona rápidamente hacia la RGB y finalmente a la rama de enfriamiento nuevamente, en la cual las escalas evolutivas nuevamente se alargan de manera que la difusión comienza nuevamente a tener efectos considerables sobre las abundancias químicas. Esto contrasta con el caso en que la difusión no es considerada, ya que en ese caso las abundancias superficiales de la estrella están determinadas por la evolución pre-WD y por la estratificación resultante del último episodio de mezcla convectiva, pero quedan congeladas durante la evolución sucesiva en la rama de enfriamiento. Otra característica importante es la que puede observarse en el panel (f) de la figura 3.6 que muestra que a bajas $\mathrm{T}_{\text {eff }}$ una fracción de helio es llevado hacia la superficie por efectos de la convección. 

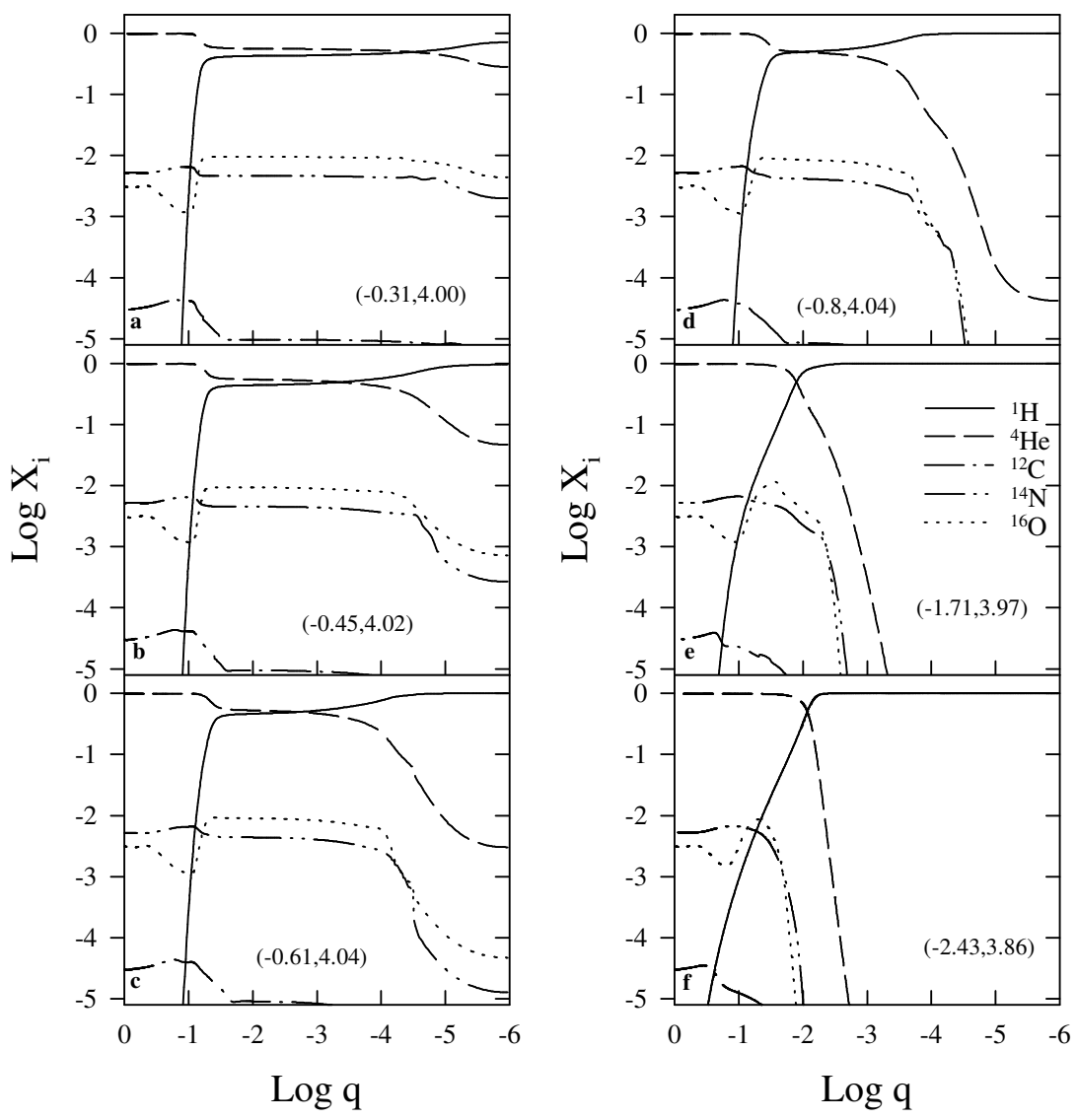

Figura 3.7: Igual que figura 3.5 pero para el modelo de $0.169 \mathrm{M}_{\odot}$. El panel (a) muestra la estratificación química antes de que el modelo entre en la rama de enfriamiento, mientras que los otros panels muestran la evolución de los perfiles de abundancias a lo largo de la curva de enfriamiento. La difusión es muy eficiente para modificar la composición de las capas externas de la estrella, aún para temperaturas efectivas elevadas, debido a las escalas de tiempo evolutivas largas de esta fase evolutiva.

Si la envoltura de hidrógeno es lo suficientemente delgada como para que la zona convectiva externa que se desarrolla a bajas $\mathrm{T}_{\text {eff }}$ alcance las capas subyacentes ricas en helio, entonces existirá una etapa evolutiva durante la cual la envoltura de la HeWD esté caracterizada por una mezcla de hidrógeno y helio. Este efecto, presente solamente en las HeWDs con masas que sufren flashes termonucleares pero con masas menores a $0.25 \mathrm{M}_{\odot}$, dura alrededor de 2 Gyr.

Cuando existe una envoltura convectiva, con composición homogénea, la difusión "opera" en la base de esta envoltura, donde las escalas de tiempo características de la difusión son mucho más largas. Esto puede tener consecuencias importantes respecto de la composición química de las envolturas de las HeWDs a bajas $\mathrm{T}_{\text {eff }}$. Si una HeWD con una envoltura convectiva acreta materia (ésta puede ser interestelar, de una compañera que pierde masa, etc.) con metales, estos metales permanecerán presentes en la envoltura durante una escala de tiempo apreciable. Los tiempos característicos para la presencia de metales en las capas externas de estas estrellas serán del orden de hasta unos 2 Gyr. Este efecto puede verse claramente en la figura 3.8, donde se muestra la evolución de la abundancia del hidrógeno $\left(X_{H}\right)$ a una profundidad de $10^{-9} \mathrm{M}_{*}$ por debajo de la superficie estelar en función de la edad para el modelo de $0.196 \mathrm{M}_{\odot}$. Durante 


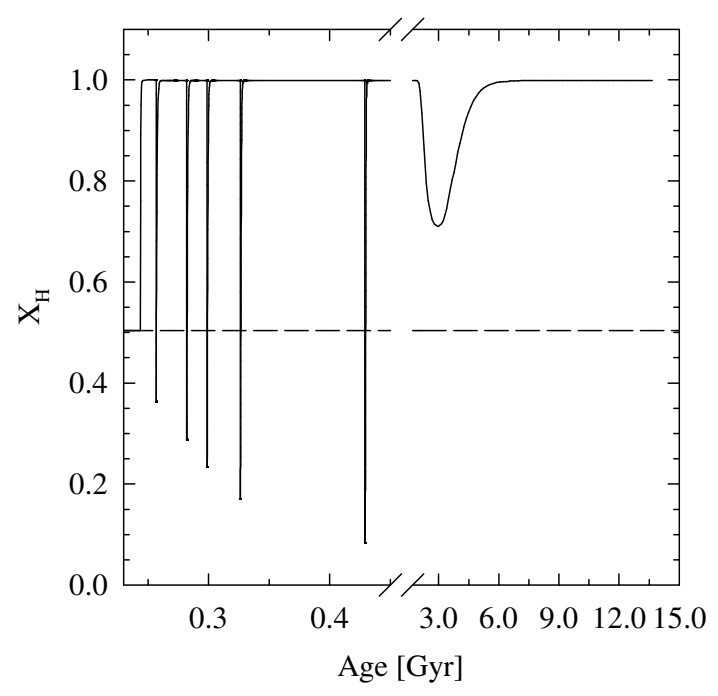

Figura 3.8: Abundancia de hidrógeno por masa (a una profundidad de $10^{-9} \mathrm{M}_{*}$ por debajo de la superficie estelar) en función de la edad para la secuencia de HeWD de $0.196 \mathrm{M}_{\odot}$. La línea sólida corresponde al caso con difusión y la línea de rayas al caso en que ésta es despreciada. Durante cada episodio de flash la abundancia de hidrógeno es reducida como resultado de la mezcla convectiva, pero la pureza de las capas externas es rápidamente restablecida por la difusión. Notar que a etapas avanzadas de la evolución y durante unos 2 Gyr, los modelos presentan capas superficiales compuestas por hidrógeno y helio. En efecto, la envoltura de hidrógeno resultante de los episodios de flash resulta suficientemente delgada como para que la zona convectiva que la HeWD desarrolla a bajas $\mathrm{T}_{\text {eff }}$ alcance zonas internas ricas en helio.

cada episodio de flash, la zona convectiva externa se hunde bien dentro de la estrella, alcanzando regiones ricas en helio, arrastrando a éste hacia la superficie y produciendo envolturas compuestas por en mezcla de hidrógeno y helio. En el último flash de este modelo, p.ej., la abundancia superficial de hidrógeno cae desde $X_{H}=1$ a $X_{H}=0.083$. El tiempo necesario para que la envoltura vuelva a ser de hidrógeno puro es en estas etapas evolutivas extremadamente corto, dada la escala de tiempo corta en que opera la difusión en las zonas externas de la estrella cuando ésta vuelve a la rama de enfriamiento. Sin embargo, en etapas evolutivas avanzadas, durante unos 2 Gyr, y como resultado del mecanismo explicadado anteriormente, los modelos están caracterizados por una abundancia $X_{H} \approx 0.75$ en su envoltura. A medida que la evolución prosigue, la convección alcanza regiones degeneradas y comienza a retroceder hacia la superficie. La degeneración comienza a ser notoria cada vez más cerca de la superficie a medida que la estrella se enfría. El espesor máximo de la zona convectiva es $M_{\text {conv }} / M_{*}=10^{-2.76}$. Si la difusión es despreciada, por el contrario, la abundancia superficial de hidrógeno permanece constante e igual a $X_{H}=0.504$.

La influencia de la difusión en las gravedades superficiales pueden verse en las figuras 3.9 a 3.12. Como ya se mencionó, la sedimentación gravitatoria hace que el hidrógeno flote y los demás elementos químicos se hundan, haciendo que la estrella aumente su radio por la disminución del peso molecular de su envoltura. Además de la masa total de la estrella y la composición química de su envoltura, es importante notar que el radio estelar dependerá de cuál es el espesor de la envoltura, es decir, de cuánto hidrógeno queda remanente cuando 


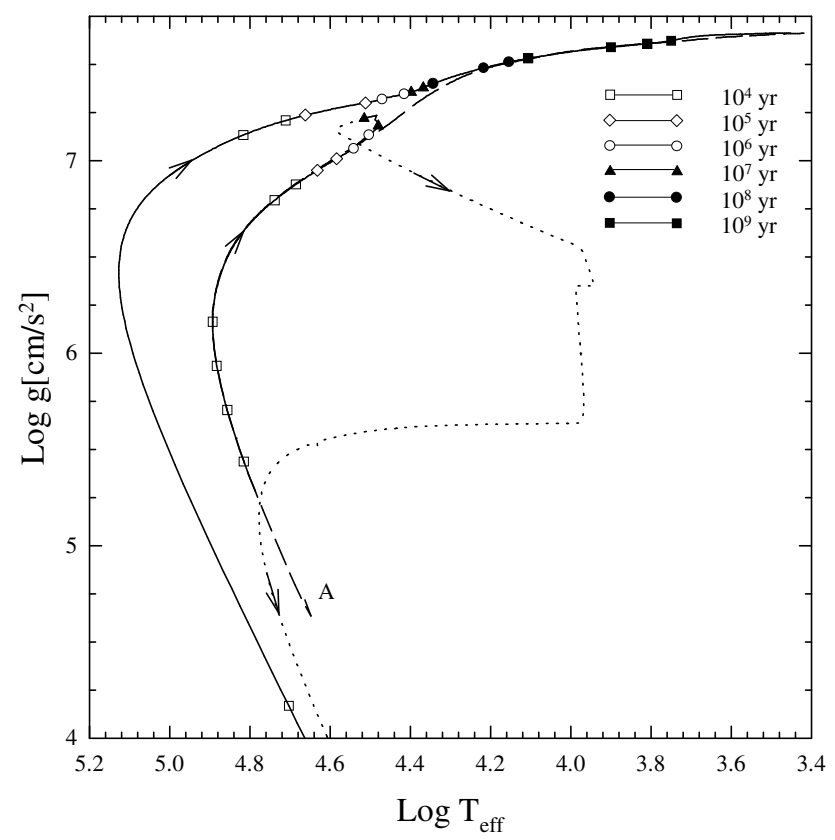

Figura 3.9: Gravedad superficial contra $\mathrm{T}_{\text {eff }}$ para la secuencia de $0.406 \mathrm{M}_{\odot}$. La línea sólida corresponde a la secuencia con difusión y la rayada a la secuencia sin difusión. Ambas secuencias comienzan en el punto denotado por "A". La evolución durante el flash se muestra en línea punteada. Símbolos adyacentes a lo largo de la curva denotan tiempos evolutivos entre los puntos indicados. El efecto del flash inducido por la difusión es claro a altos valores de $\mathrm{T}_{\text {eff }}$. Sin embargo, excepto por estas rápidas etapas evolutivas, las gravedades superficiales no son modificadas apreciablemente por la inclusión de los efectos de la difusión.

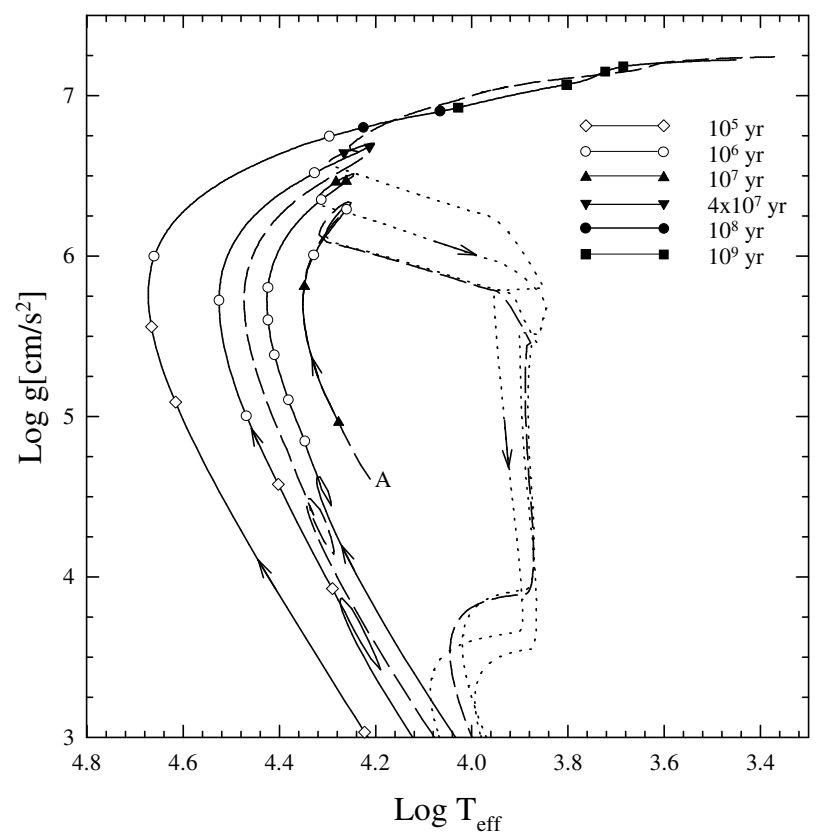

Figura 3.10: Igual a figura 3.9 pero para la secuencia de $0.242 \mathrm{M}_{\odot}$. 


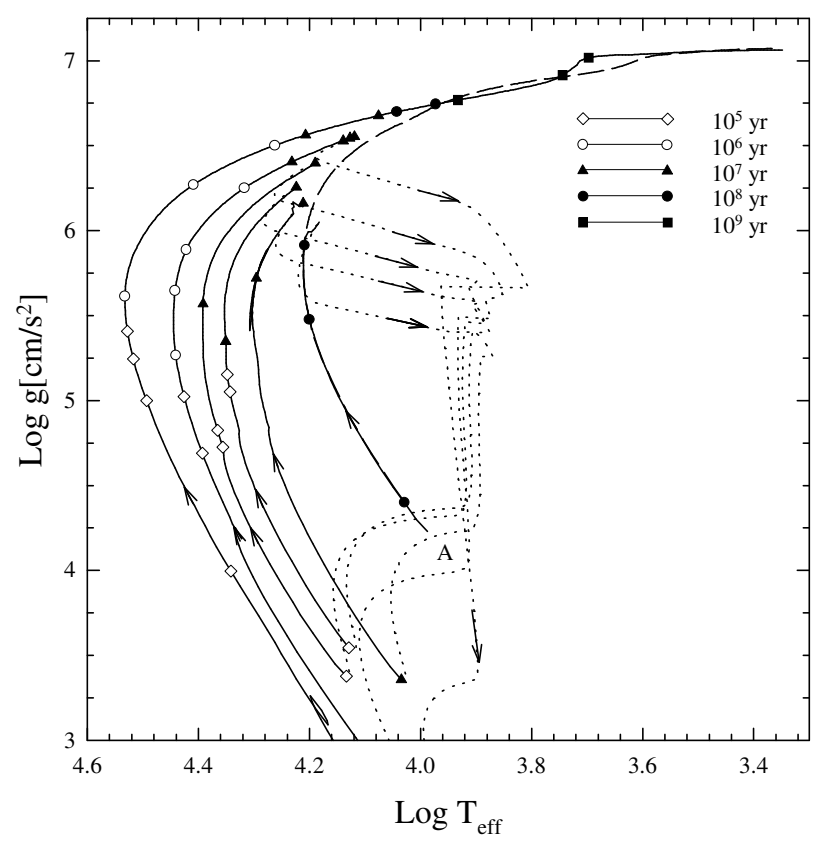

Figura 3.11: Igual a figura 3.9 pero para la secuencia de $0.196 \mathrm{M}_{\odot}$.

la estrella entra en la rama de enfriamiento. En vista de estas consideraciones, es razonable esperar entonces que la relación masa-radio se vea afectada por la inclusión de la difusión. Sin embargo, una inspección cuidadosa a las figuras 3.9 a 3.11 muestra que hasta que comienzan los flashes termonucleares, la gravedad de los modelos con y sin difusión son prácticamente iguales para una masa estelar dada. La evolución subsiguiente difiere marcadamente entre los modelos con y sin difusión y esto se aprecia claramente en las figuras, donde las etapas correspondientes a los loops inducidos por los flashes termonucleares se muestran en líneas punteadas.

Una vez que estas etapas han finalizado puede verse que los modelos con y sin difusión convergen a un mismo valor de gravedad superficial para un valor de $\mathrm{T}_{\text {eff }}$ dado. Este resultado es la consecuencia de dos efectos producidos por la difusión que se compensan uno con otro. Por un lado, como se dijo, la difusión produce envolturas con menor peso molecular promedio y por lo tanto más extendidas. Por el otro, durante los flashes inducidos por la difusión, la envoltura de hidrógeno es consumida en gran parte, por lo que cuando la estrella entra en su rama de enfriamiento final, la cantidad de hidrógeno remanente es mucho menor que en el caso sin difusión. Estos dos efectos se compensan mutuamente y dan como resultado una relación masa-radio similar para los modelos con y sin difusión. En el caso de los modelos de baja masa que no sufren flashes, los resultados se muestran conjuntamente en la figura 3.12. La situación es ahora marcadamente diferente entre los modelos que incluyen difusión y los estándar. Luego de la evolución pre-WD, la difusión opera de manera eficiente, en escalas de tiempo más cortas que las que caracterizan la evolución de estos modelos. De esta manera, la estrella sufre una expansión de sus capas externas pronunciada y la temperatura en la base de la envoltura de hidrógeno se reduce, disminuyendo la tasa de liberación de energía nuclear. El resultado es que menos hidrógeno es consumido en relación a los modelos sin difusión, contrariamente al caso de los modelos más masivos. De esta manera, cuando la estrella entra en la rama de enfriamiento tiene una envoltura de hidrógeno puro y además más masiva que los 


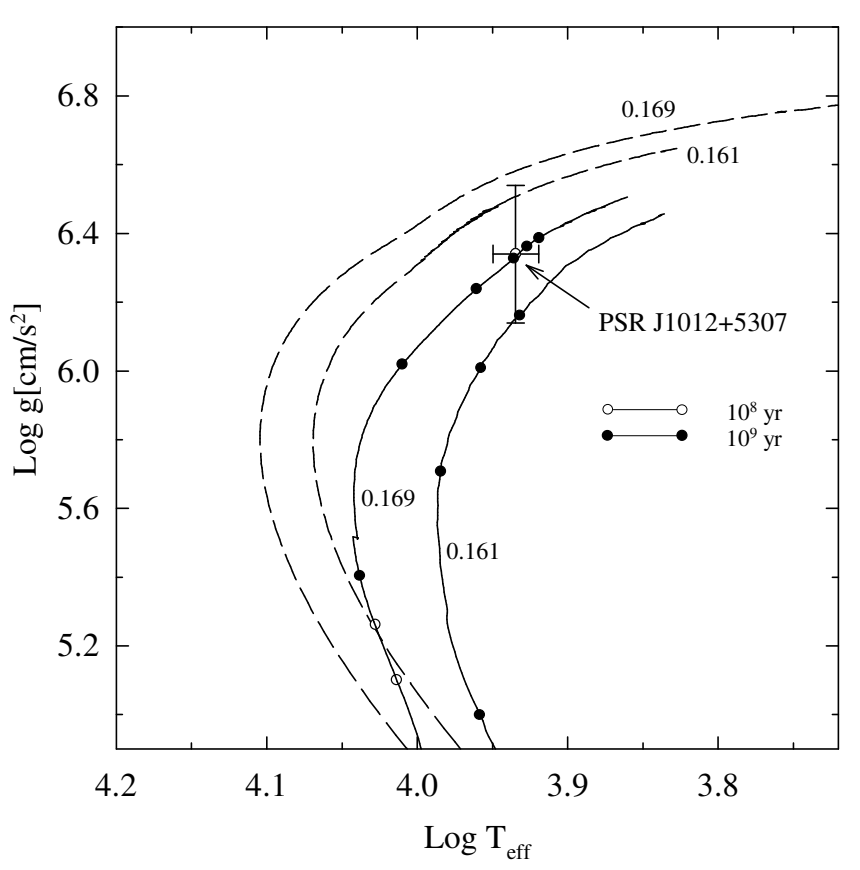

Figura 3.12: Igual a figura 3.9 pero para la secuencia de $0.169 \mathrm{M}_{\odot}$. En este caso los modelos no experimentan ningún flash termonuclear aún cuando se incluye la difusión. La ubicación de la compañera del púlsar PSR J1012+5307 (Callanan, Garnavich, Koester, 1998) también está indicada. Los modelos en que la difusión está considerada están caracterizadas por gravedades superficiales considerablemente más bajas que en los modelos sin difusión.

modelos sin difusión de igual masa. La consecuencia es clara en la figura 3.12, donde se puede apreciar que la gravedad superficial de los modelos con difusión es marcadamente más baja (hasta en un 80\%) que cuando ésta es despreciada. En esta misma figura se incluyen los datos observacionales de la HeWD compañera del PSR J1012+5307 de acuerdo a la determinación de Callanan, Garnavich, Koester (1998). Es claro de esta figura que las variaciones en la relación masa-radio que induce la difusión deben ser tenidos en cuenta al interpretar las observaciones. La masa derivada a partir de nuestros modelos para la compañera de PSR J1012+5307 es de $0.17 \pm 0.01 \mathrm{M}_{\odot}$, mientras que los modelos sin difusión predicen un valor de $0.15 \pm 0.01 \mathrm{M}_{\odot}$ (Driebe et al. 1998 y nuestros resultados sin difusión). En las figuras 3.9 a 3.12 también se presentan los tiempos evolutivos de los modelos con difusión. Desde el punto de vista de la interpretación de las observaciones esto resulta importante ya que la velocidad de la evolución determina la probabilidad de hallar a una HeWD en una etapa evolutiva determinada. Las etapas caracterizadas por una gravedad superficial baja son etapas de evolución rápida, ya que corresponden a las fases de contracción de la HeWD después de la ocurrencia de un flash y es por lo tanto muy poco probable hallar HeWDs caracterizadas por estas gravedades. Sobre este punto, directamente relacionado con la función de luminosidad, volveremos en el capítulo 4. Consideremos ahora las escalas de tiempo evolutivas características de las HeWDs. Como se ha mencionado anteriormente, la rapidez de la evolución de las HeWDs en la rama de enfriamiento estará determinada básicamente por la importancia de la liberación de energía nuclear a través de la fusión del hidrógeno vía la cadena protón-protón. A su vez, esto está determinado por el espesor de la envoltura de hidrógeno de las HeWDs. En la figura 3.13 se muestra la masa de 
$\log \left(\mathrm{M}_{\mathrm{H}} / \mathrm{M}_{*}\right)$ vs. Age [Gyr]
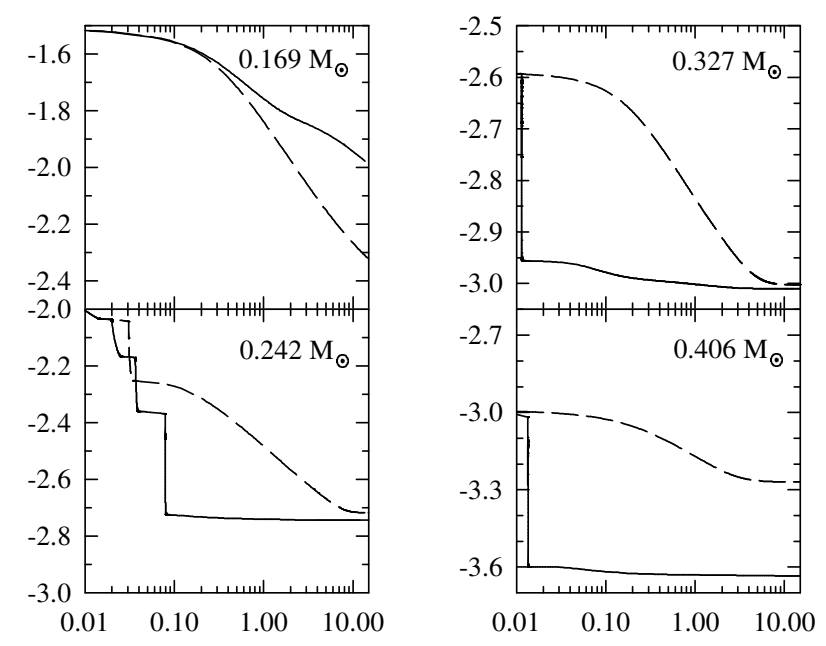

Figura 3.13: Masa de la envoltura de hidrógeno contra edad. Las masas de los modelos se indican en cada panel. Modelos con difusión: línea sólida; modelos sin difusión: línea rayada. El modelo de masa más baja, que no sufre ningún flash termonuclear, resulta con una envoltura de hidrógeno más masiva que el modelo sin difusión. Los modelos que sufren flashes están caracterizados después de los episodios de flash, al entrar en la rama de enfriamiento, por envolturas mucho más delgadas que los modelos sin difusión.

la envoltura de hidrógeno en función de la edad de la estrella para los modelos de 0.406, 0.327, 0.242 у $0.169 \mathrm{M}_{\odot}$.

La característica más importante es que todos los modelos que sufren flashes termonucleares quedan con envolturas de hidrógeno mucho más delgadas que los modelos respectivos sin difusión después de la etapa de los flashes. En efecto, en los modelos sin difusión, cuando las estrellas entran en la rama de enfriamiento, las envolturas resultan entre 2 y 4 veces más masivas que cuando la difusión es considerada. Esto es así aún para el modelo de $0.242 \mathrm{M}_{\odot}$, que sufre un flash aún en ausencia de la difusión. La característica general que puede apreciarse es que el hidrógeno es consumido fuertemente durante los flashes, sin embargo, el único flash que sufre el modelo estándar de $0.242 \mathrm{M}_{\odot}$ no es capaz de reducir la envoltura en la misma medida en que es consumida cuando la difusión es tenida en cuenta. Puede verse entonces que los modelos con difusión tienen envolturas muy delgadas durante su evolución en la rama de enfriamiento. Esto tiene consecuencias directas en las escalas de tiempo evolutivas. El modelo de $0.169 \mathrm{M}_{\odot}$, por el contrario, muestra un comportamiento inverso. La difusión, en este caso, no es capaz de inducir flashes en el shell de hidrógeno y su efecto más importante es producir en expansión de las zonas externas de la estrella, por cambios en el peso molecular promedio, como ya se ha dicho antes. Esta expansión provoca un enfriamiento de las base de la envoltura, donde las reacciones nucleares tienen lugar y como consecuencia la eficiencia con que el hidrógeno es consumido disminuye también. De esta manera, este modelo está caracterizado por envolturas más masivas cuando los efectos de la difusión son tenidos en cuenta. Las escalas evolutivas en la rama de enfriamiento serán similares para esta masa estelar para los modelos con y sin 
$\log \left(\mathrm{L}_{\text {nuc }} / \mathrm{L}\right)$ vs. Age $[\mathrm{Gyr}]$
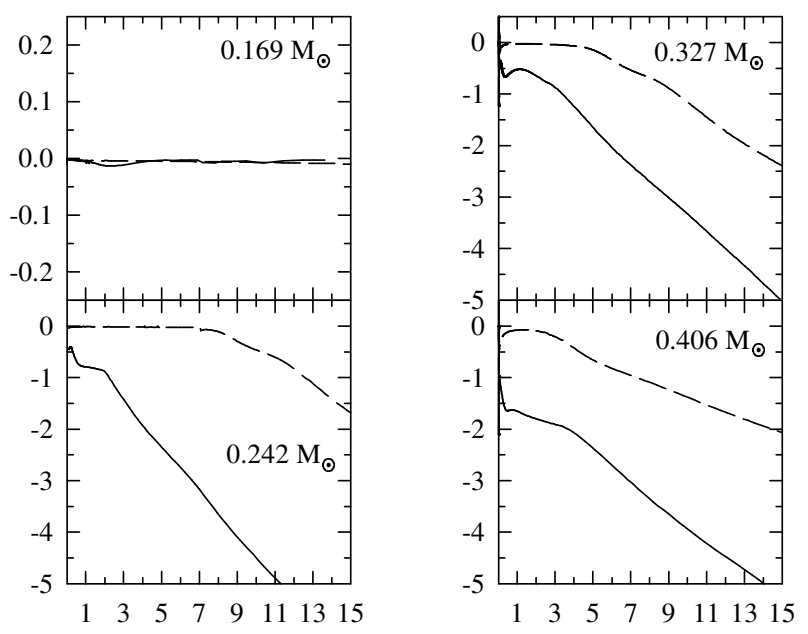

Figura 3.14: Cociente entre las luminosidades nuclear y superficial como función de la edad. Los valores de la masa de los modelos se indican en cada figura. Modelos con difusión: línea sólida; modelos sin difusión: línea rayada. Excepto para la masa estelar más pequeña que no experimenta flashes termonucleares, la liberación de energía nuclear es despreciable para los modelos con difusión a lo largo de la curva de enfriamiento. Sin embargo, para los modelos sin difusión, la combustión del hidrógeno es la fuente de energía dominante aún en estados avanzados de la evolución, con el resultado de que la evolución en estos casos es lenta.

difusión.

La importancia relativa de la energía nuclear como fuente de energía para la estrella se muestra en la figura 3.14. Para los modelos con y sin difusión, y las mismas masas que en la figura 3.13, se ilustra en escala logarítmica el cociente entre la luminosidad producida por liberación de energía nuclear y la luminosidad superficial de la estrella en función de la edad de los modelos. El alcance de los efectos de la difusión en el espesor de las envolturas de hidrógeno resultantes pueden seguirse hasta esta figura. Por claridad las etapas de flashes no han sido incluidas. En el caso menos masivo la energía nuclear aporta prácticamente el total de la energía emitida por la estrella, resultado que es consecuencia de la envoltura de hidrógeno masiva y coincidente con el caso estándar. Por el contrario, los modelos que sufren flashes muestran comportamientos claramente diferenciados. Como resultado de las envolturas delgadas, en los modelos con difusión la energía nuclear apenas contribuye al total de energía empleado por la estrella. Además, después de los 2 Gyr esta contribución, inicialmente pequeña, cae rápidamente siendo durante casi toda la evolución de los modelos completamente despreciable. Si la difusión es despreciada, la energía nuclear contribuye apreciablemente durante una escala de tiempo mucho más larga, dando una evolución mucho más lenta en la rama de enfriamiento (ver también Driebe et al., 1998). Por ejemplo, consideremos el modelo de $0.242 \mathrm{M}_{\odot}$. En el caso con difusión, la contribución de la energía nuclear al total radiado por la estrella decae a un $10 \%$ a los 3 Gyr y a un $1 \%$ a los 4 Gyr. Por el contrario, en el caso sin difusión, aporta prácticamente el 100\% de la energía radiada hasta los 7 Gyr de edad, y sólo entonces declina, y 
$\log \mathrm{T}_{\text {eff }}$ vs. Age [Gyr]
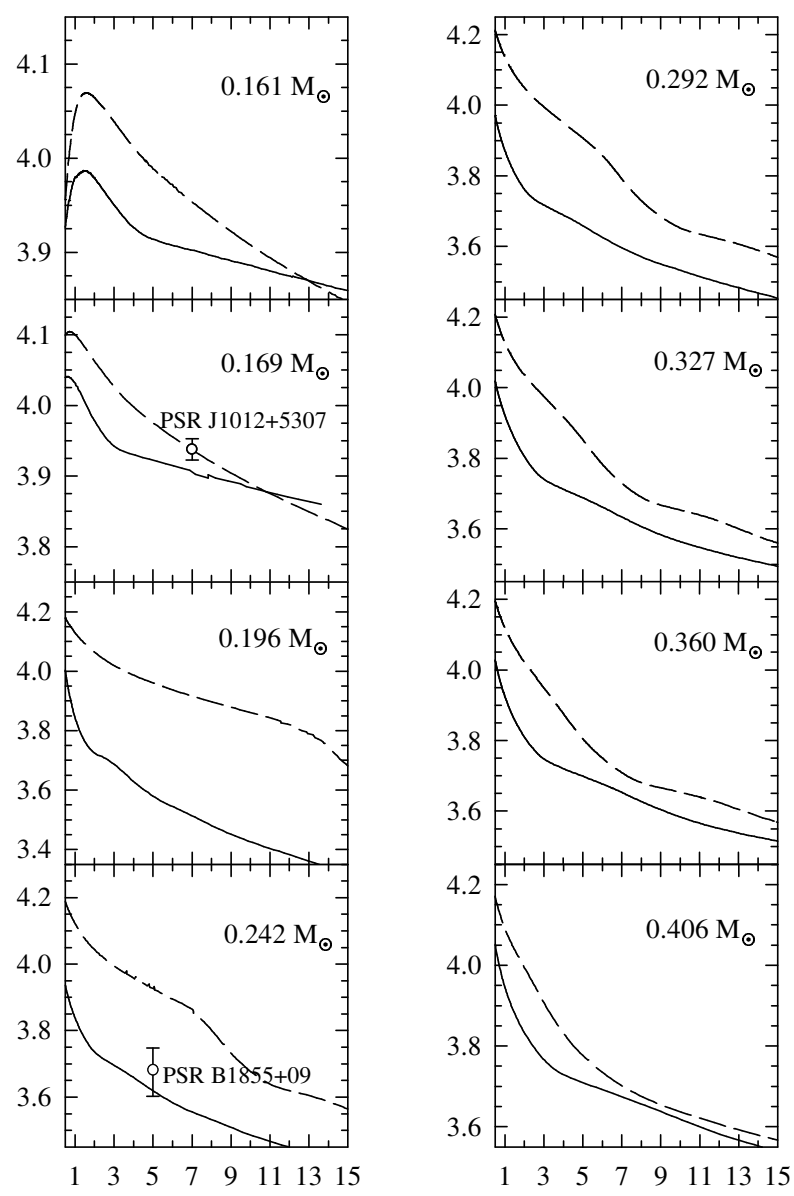

Figura 3.15: $\mathrm{T}_{\text {eff }}$ contra edad para todos los modelos de HeWD computados. Las líneas sólidas representan los modelos sin difusión y las rayadas los modelos con difusión. Se incluyen los datos observacionales de las HeWDs compañeras de PSR J1012+5307 y PSR B1855+09.

de manera paulatina, alcanzando un $10 \%$ a los 11 Gyr y no llegando al $1 \%$ ni aún en 15 Gyr. Las implicancias de los resultados presentados pueden apreciarse analizando la rapidez con que las HeWDs se enfrían, lo que puede verse en la figura 3.15, donde se muestra la $T_{\text {eff }}$ en función de la edad de los modelos. En estos gráficos se incluyen los datos observacionales para las HeWDs compañeras de PSR J1027+5307 y PSR B1855+09 (Callanan, Garnavich, Koester, 1998 y van Kerkwijk et al., 2000 respectivamente), asumiendo que la edad de estas HeWD es igual a la edad de spin-down del púlsar. Es importante destacar que nuestros modelos sin difusión están en buen acuerdo con los de Driebe et al. (1998) en base a las mismas suposiciones. En este caso, la evolución está dominada por la combustión residual del hidrógeno, dando lugar a edades de enfriamiento grandes. En particular, estos modelos predicen una edad para la HeWD en PSR J1012+5307 en buen acuerdo con la edad de spin-down del púlsar ( $\approx 7$ Gyr). Sin embargo, para la compañera de PSR B1855+09 estos modelos predicen una edad de $10 \mathrm{Gyr}$, mientras que la edad del púlsar es de 5 Gyr.

Consideremos ahora las edades de enfriamiento de los modelos con difusión. Vimos que cuando los modelos sufren flashes termonucleares (masas mayores a $\approx 0.18 \mathrm{M}_{\odot}$ ) la masa de 
hidrógeno de la envoltura que queda cuando éstos entran en la rama de enfriamiento es muy pequeña y por lo tanto la liberación de energía nuclear es despreciable. En este caso, la HeWD debe obtener la energía de su contenido térmico, con el resultado de que las edades de enfriamiento son marcadamente más reducidas que en el caso sin difusión. Por ejemplo, para la compañera de PSR B1855+09, los modelos con difusión predicen una edad de $4 \pm 2$ Gyr, en buen acuerdo con la edad del púlsar. Para masas menores que $0.18 \mathrm{M}_{\odot}$, la combustión del hidrógeno es la fuente de energía dominante y las edades son similiares a las obtenidas sin difusión. De este modo, los modelos con difusión predicen naturalmente un buen acuerdo entre las edades de las HeWDs y las edades de spin-down de los púlsares binarios mencionados. Otros autores han introducido episodios de pérdida de masa ad-hoc (Schönberner, Driebe \& Blöcker, 2000) para obtener masas de envolturas de hidrógeno reducidas que permitan un enfriamiento rápido de los modelos de HeWDs. Sin embargo consideremos el caso de PSR J1713+0747, cuya compañera WD tiene una masa estimada de $\approx 0.33 \mathrm{M}_{\odot}$ en base a la relación de Tauris \& Savonije (1999). En este caso los modelos de Schönberner, Driebe \& Blöcker (2000) no predicen la ocurrencia de flashes termonucleares por lo que una vez formada la HeWD no puede ocurrir un episodio de pérdida de masa adicional, de manera que la envoltura de hidrógeno no puede reducirse de esta manera. Nuestros modelos, sin embargo, predicen naturalmente la ocurrencia de un flash termonuclear para una HeWD con esta masa, por lo que su envoltura de hidrógeno puede ser reducida bien por combustión nuclear como también por pérdida de masa adicional (durante la excursión de la estrella a la RGB como resultado de la expansión producida por el flash nuclear). De esta manera, la difusión de elementos se revela como clave para la correcta interpretación de las edades de enfriamiento de las HeWDs.

\subsubsection{Evolución durante un flash termonuclear}

En base a los resultados de la sección anterior, resulta claro que los flashes termonucleares tienen una influencia decisiva en la evolución de las HeWDs. En este sentido, resulta útil presentar una descripción pormenorizada de la evolución de las HeWDs durante estas etapas.

Para ejemplificar la evolución de las HeWDs durante los flashes termonucleares inducidos por la difusión seleccionamos, del modelo de $0.242 \mathrm{M}_{\odot}$, la fase evolutiva que va desde un poco antes de la ocurrencia del segundo flash termonuclear hasta un poco después del mismo, cerca del comienzo del tercer flash. En la figura 3.16 mostramos esta parte del track evolutivo en el HRD. A lo largo del track se especifican los tiempos transcurridos hasta y desde el máximo de liberación de energía nuclear $\left(L_{\text {nuc }}\right)$. Se indican también los números de algunos modelos que serán empleados posteriormente para mostrar la evolución del perfil de abundancias de hidrógeno. Una vez que el flash comienza, la evolución en el HRD es muy rápida. El modelo 9200 corresponde a $10^{3}$ años antes del máximo de $L_{\text {nuc }}$. Desde este punto la evolución se acelera y la mayor parte del trayecto hacia luminosidades altas es realizado en sólo unos cientos de años. Después de alcanzar dimensiones de gigante, la estrella evoluciona hacia el azul, contrayéndose, hasta $\log \mathrm{T}_{\text {eff }} \approx 4.2$, donde el track muestra un pequeño loop, producto de un máximo local en la liberación de energía nuclear causado por el aumento de temperatura en la base de la envoltura de hidrógeno, resultado de la rápida contracción de las capas externas de la estrella. A partir del modelo 22000 aproximadamente, la evolución de la estrella se enlentece de manera que la difusión modifica apreciablemente los perfiles de abundancias en las capas externas de la estrella. Alrededor de 3 Myr después del máximo de $L_{\text {nuc }}$, la cola del perfil de hidrógeno se difunde hacia el interior de la estrella (por efectos de la difusión química) dando lugar de esta manera a la ocurrencia del tercer flash termonuclear que sufre este modelo. 


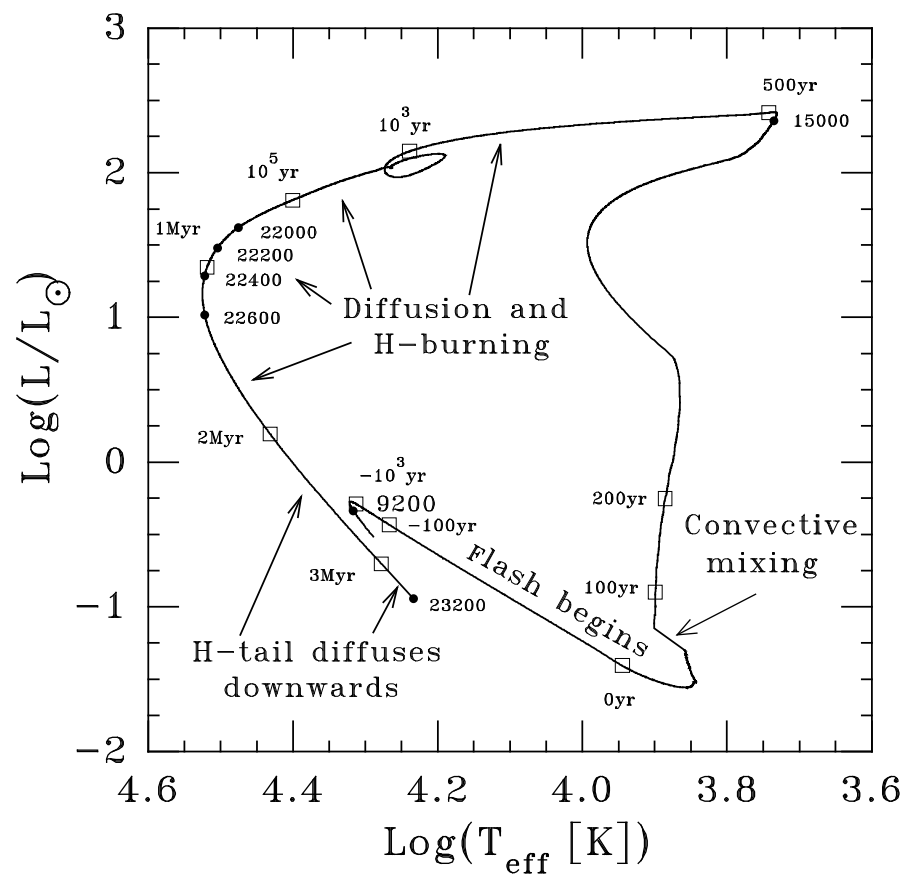

Figura 3.16: Track evolutivo del modelo de $0.242 \mathrm{M}_{\odot}$ con difusión durante el segundo flash termonuclear. Los tiempos indicados por cuadrados a lo largo del track corresponden a tiempos antes y después del máximo de liberación de energía nuclear $L_{\text {nuc }}$ (que define el punto de edad cero en esta figura). Algunos modelos particulares están marcados con círculos y el número de modelo correspondiente a la secuencia calculada.

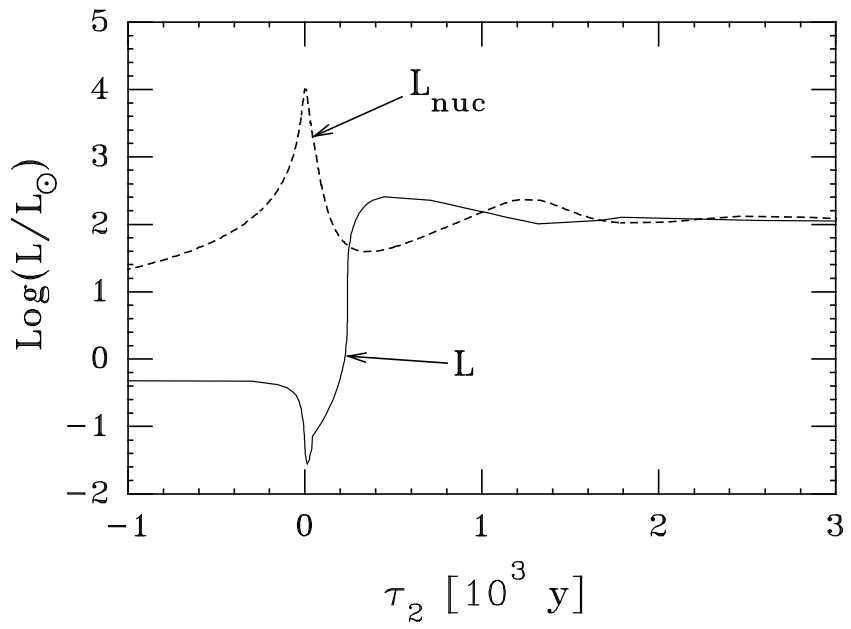

Figura 3.17: Luminosidades nuclear y superficial en función de tiempo para el modelo de $0.242 \mathrm{M}_{\odot}$ con difusión. La etapa evolutiva mostrada corresponde a un intervalo de tiempo corto durante el segundo flash termonuclear. El punto cero de la edad está fijado en el momento de máxima $L_{\text {nuc }}$. 


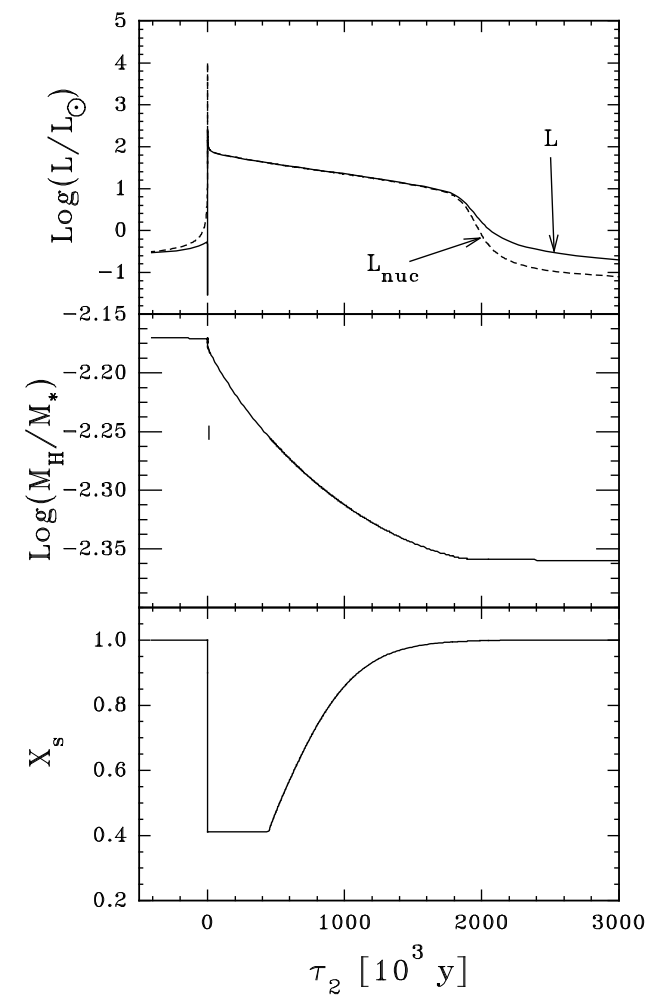

Figura 3.18: Características relevantes del modelo de $0.242 \mathrm{M}_{\odot}$ con difusión durante el segundo flash termonuclear. Panel superior: evolución de las luminosidades nuclear y superficial. El pico es debido a la ocurrencia del flash termonuclear. Panel medio: evolución de la masa de la envoltura de hidrógeno. Panel inferior: abundancia por fraccón de masa de hidrógeno a $q=10^{-9}$. Poco después del flash, el modelo desarrolla una zona convectiva externa que produce la mezcla del hidrógeno y el helio, que reduce marcadamente la abundancia superficial de hidrógeno. Sin embargo, la sedimentación gravitatoria produce rápidamente una envoltura pura de hidrógeno nuevamente.

En la figura 3.17 se muestran la luminosidad nuclear y superficial durante la primera etapa del segundo flash termonuclear. Aquí, $\tau_{2}$ es la edad del modelo medida desde el momento en que $L_{\text {nuc }}$ alcanza su máximo. Puede verse en esta figura que el máximo en la luminosidad superficial se alcanza unos 500 años después que el máximo de $L_{\text {nuc }}$. Para más detalles del comportamiento de este objeto durante estas fases, pueden verse en la figura 3.18 la evolución de la luminosidad superficial, nuclear, la masa de la envoltura de hidrógeno y la abundancia de hidrógeno por fracción de masa como función de $\tau_{2}$.

Consideremos la evolución del perfil de abundancia del hidrógeno durante este flash termonuclear. Los efectos de la difusión son notables en dos sentidos. Por un lado, en la superficie, la sedimentación gravitatoria tiende a aumentar la abundancia de hidrógeno haciendo que los elementos más pesados se hundan. En la base de la envoltura de hidrógeno, por el contrario, existe un gradiente de abundancias grande por lo que la difusión química, que actúa en contra de este gradiente, resulta efectiva.

De esta manera una parte del hidrógeno en estas capas de la estrella se hunde, alcanzando 


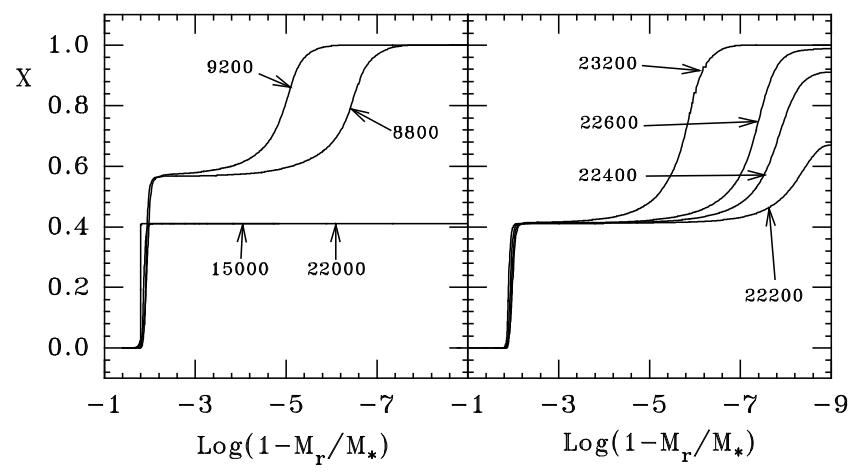

Figura 3.19: Perfiles de hidrógeno para algunos modelos seleccionados indicados por el número de modelo (ver figura 3.16).

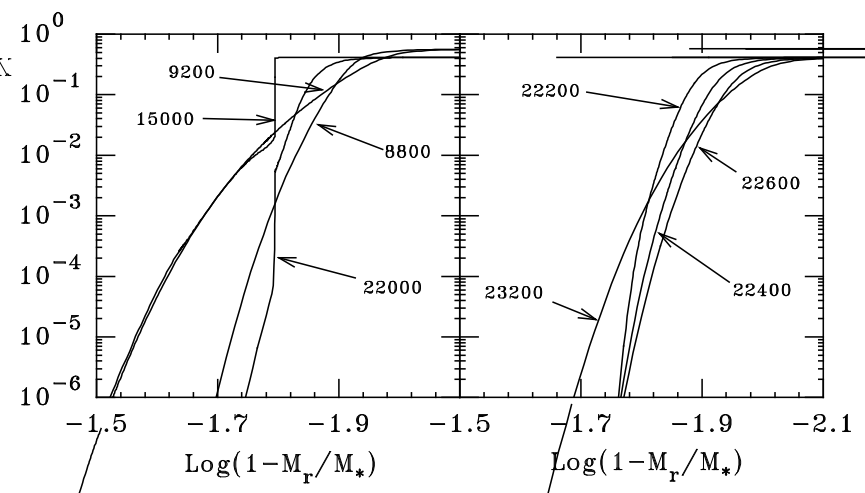

Figura 3.20: Igual que en la figura 3.19 pero para la cola de la distribución de hidrógeno.

regiones de la estrella que están lo suficientemente calientes como para que se las reacciones que queman el hidrógeno se enciendan y, en un momento determinado, produzcan un flash termonuclear. Si bien es cierto que un flash de estas características puede ocurrir aún en ausencia de la difusión (recordar que la secuencia de $0.242 \mathrm{M} \odot$ sin difusión sufre un flash termonuclear), una vez ocurrido este flash, en ausencia de difusión no existe un mecanismo capaz de alimentar nuevamente las capas internas de la estrella con combustible fresco. Esta es la diferencia clave entre las secuencias de modelos con y sin difusión.

En la figura 3.19 se muestran los perfiles de hidrógeno para modelos que van desde el comienzo del flash (modelo 8800) hasta momentos antes de que un nuevo flash se produzca (modelo 23200). Del modelo 8800 al 9200 puede verse como el hidrógeno se difunde hacia afuera por efectos de la sedimentación gravitatoria, hasta que por efectos del flash se desarrolla una zona convectiva externa que mezcla el hidrógeno con helio de capas más internas de la estrella (modelos 15000 y 22000). Cuando el modelo retorna al azul y la evolución se enlentece nuevamente (panel derecho), la sedimentación gravitatoria vuelve a ser importante dando lugar a envolturas puras de hidrógeno.

En la figura 3.20 se muestran los detalles del perfil de hidrógeno en la base de la envoltura 
para los mismos modelos que en la figura 3.19. Desde el modelo 8800 al 9200 la difusión química lleva parte del hidrógeno hacia partes más internas de la estrella, suficientemente calientes como para que el hidrógeno se encienda de manera inestable, produciendo el flash termonuclear. Después el objeto desarrolla una zona convectiva externa, que mezcla el hidrógeno y el helio, produciendo el perfil tipo "escalón" que puede verse para el modelo 15000. El perfil del modelo 22000 muestra claramente el efecto de la combustión nuclear subsiguiente, ya que la abundancia de hidrógeno se ha reducido notablemente. En el panel derecho puede verse la evolución subsiguiente del perfil de hidrógeno. La secuencia de modelos 22200-22400-22600 muestra cómo el hidrógeno disminuye por efectos de la reacciones nucleares y por último, el modelo 23200 muestra cómo la difusión química nuevamente lleva la cola de la distribución hacia el interior de la estrella, lo que dará lugar a un nuevo episodio de flash.

\subsection{Discusión y conclusiones}

Motivados por recientes observaciones de enanas blancas de baja masa compañeras de púlsares de milisegundo (van Kerkwijk et al., 2000), que ponen en duda los espesores de las envolturas de hidrógeno predichas por cálculos evolutivos estándar (Driebe et al., 1998; Sarna, Ergma \& Antipova, 2000), hemos realizado un conjunto de cálculos evolutivos para investigar los efectos de la difusión en la ocurrencia de flashes termonucleares en el shell de hidrógeno en HeWDs y, más importante aún, para investigar si la envoltura de hidrógeno puede ser reducida o no por la combustión del hidrógeno durante estos episodios de flash. Para este fin hemos explorado la evolución de modelos de HeWD de 0.406, 0.360, 0.327, 0.292, 0.242, 0.196, 0.169 y $0.161 \mathrm{M}_{\odot}$ de manera auto-consistente con los efectos de los procesos de difusión, que incluyen la sedimentación gravitatoria, la difusión química y la térmica. Para poder realizar una comparación consistente que permite dilucidar los efectos de la difusión, los mismos modelos han sido evolucionados pero despreciando estos procesos. En este sentido, nuestros cálculos sin difusión dan resultados similiares a los obtenidos por Driebe et al. (1998). Hay que mencionar también que en nuestros cálculos no hemos invocado episodios de pérdida de masa cuando los modelos alcanzan dimensiones de estrellas gigantes como resultado de los episodios de flash porque nuestro principal interés es analizar los efectos de la difusión. En este aspecto, nuestros cálculos difieren del presentado por Iben \& Tutukov (1986) para un modelo de HeWD de $0.296 \mathrm{M}_{\odot}$, donde un fuerte episodio de pérdida de masa causa una disminución apreciable de la envoltura de hidrógeno y contribuye a que el objeto evolucione rápidamente en la rama de enfriamiento.

De los resultados presentados aquí, es claro que la difusión de elementos afecta considerablemente la estructura y evolución de las HeWDs, y lleva a una diferente historia de enfriamiento para estos objetos. Hemos encontrado que los modelos con masas mayores que $\approx 0.18 \mathrm{M}_{\odot} \mathrm{e}$ incluso hasta el modelo más masivo que hemos analizado $\left(0.406 \mathrm{M}_{\odot}\right)$ experimentan al menos un flash termonuclear cuando la difusión es incorporada. Esto contrasta con los modelos que no incluyen la difusión, para los cuales sólo el modelo de $0.242 \mathrm{M}_{\odot}$ sufre un flash (Driebe et al., 1998 encuentran flashes en sus modelos de 0.234 y $0.259 \mathrm{M}_{\odot}$. La evolución subsiguiente resulta muy afectada por los flashes inducidos por difusión, ya que las envolturas de hidrógeno resultan muy reducidas, haciendo que la combustión nuclear estable residual durante la rama de enfriamiento sea despreciable como fuente de energía de la estrella. Dado entonces que la estrella tiene una cantidad de energía disponible mucho menor, esto implica que las escalas de tiempo evolutivas serán mucho más cortas comparadas a las obtenidas en el caso de modelos sin difusión. 
Estos resultados tienen importantes implicancias cuando se comparan las predicciones teóricas sobre la evolución de las HeWD con los datos inferidos a partir de observaciones de púlsares de milisegundo. Esto es particularmente importante en el caso del púlsar PSR B1855+09. van Kerkwijk et al. (2000) determinaron la $T_{\text {eff }}$ de la HeWD compañera, que es $4800 \pm 800 \mathrm{~K}$, y ya que la masa de esta HeWD es conocida con precisión $\left(M_{W D}=0.258_{-0.016}^{+0.028} \mathrm{M}_{\odot}\right.$; Kaspi, Taylor \& Ryba, 1994), este bajo valor de $\mathrm{T}_{\text {eff }}$ corresponde a una edad de enfriamiento de 10 Gyr en el caso de los modelos sin difusión. Este valor está en discrepancia con la edad de spin-down del púlsar, que es 5 Gyr. Por el contrario, los modelos con difusión predicen una edad de $4 \pm 2$ Gyr, debido al aceleramiento que sufre la evolución de las HeWDs por efectos de la ocurrencia de los flashes inducidos por difusión, desapareciendo de este modo la discrepancia entre las edades de ambos objetos.

En vista de estos resultados, es interesante analizar otros púlsares de milisegundo que dan resultados discrepantes con los modelos sin difusión. El sistema PSR J0034-0534 es particularmente notorio en este sentido. La enana blanca en este sistema es muy fría $\left(\mathrm{T}_{\text {eff }}<3500 \mathrm{~K}\right)$ y la edad del púlsar es $6.8 \pm 2.4$ Gyr (Hansen \& Phinney, 1998b). De acuerdo a la relación entre el período orbital del sistema y la masa de la WD (Tauris \& Savonije, 1999), la masa de la WD compañera de este púlsar es $\approx 0.21 \mathrm{M}_{\odot}$. En vista de estos valores, los modelos sin difusión predicen una edad bien por encima de los 10 Gyr para esta WD. Por el contrario, los cálculos evolutivos que incluyen la difusión de elementos dan una edad de 6 y 8 Gyr para una $\mathrm{T}_{\text {eff }}$ de 3000 y $3500 \mathrm{~K}$ respectivamente, en excelente acuerdo con la edad del púlsar. Es evidente que puede obtenerse consistencia entre las edades de la WD y el púlsar si se supone que la WD es una WD con núcleo de carbono oxígeno y una masa de $0.5 \mathrm{M}_{\odot}$ (Schönberner, Driebe \& Blöcker, 2000). Sin embargo este valor entra en franca contradicción con el valor inferido mediante la relación de Tauris \& Savonije (1999). Otro púlsar de milisegundo con una WD fría como compañera de relevancia en este contexto es PSR J1713+0747. Para la compañera de este púlsar, Hansen \& Phinney (1998b) dan una $\mathrm{T}_{\text {eff }}<3800 \mathrm{~K}$ y una edad de spin-down del sistema de $9.2 \pm 0.4$ Gyr. En este caso, la masa de la WD de acuerdo a la relación de Tauris \& Savonije $(1999)$ es $\approx 0.33 \mathrm{M}_{\odot}$. Los modelos sin difusión predicen edades mayores a 13 Gyr para esta WD, mientras que los modelos con difusión dan una edad de 9.1 Gyr, en muy buen acuerdo con la edad del púlsar. Otro púlsar interesante en este contexto es PSR J0437-4715, para el cual la edad de spin-down es de 4.9 Gyr (Baasa, comunicación privada). La masa de su compañera ha sido determinada recientemente por van Straten et al. (2001) por medio del retraso de Shapiro de la señal del púlsar y es $M_{\mathrm{WD}}=0.236 \pm 0.017 \mathrm{M}_{\odot}$ y en base a datos fotométricos (Danziger, Baade \& Della Valle, 1993) y los modelos de HeWDs presentados aquí junto con modelos de atmósferas no grises (ver Serenelli, Althaus, Rohrmann \& Benvenuto 2001; Rohrmann, Serenelli, Althaus \& Benvenuto 2002 y el capítulo 4 de esta tesis), la edad que se obtiene para la HeWD compañera de PSR J0437-4715 es de alrededor de 4.4 Gyr, en excelente acuerdo con la edad del púlsar.

Para finalizar, es claro que la difusión de elementos juega un rol muy importante en la historia de enfriamiento de las HeWDs. De los resultados del presente estudio concluimos que la discrepancia en las edades entre las predicciones de los cálculos evolutivos estándar y recientes observaciones de púlsares de milisegundo con compañeras WD parecen ser el resultado de ignorar los efectos de la difusión en los cálculos evolutivos. En efecto, tales discrepancias desaparecen cuando la difusión es incorporada en los cálculos. 


\section{Capítulo 4}

\section{Enanas blancas de baja masa II. Índices de color}

En general, el tratamiento de la estructura y evolución estelar se considera separadamente de un tratamiento detallado de la atmósfera estelar. Desde el punto de vista de la estructura de la estrella, esto está justificado en la medida en que los detalles de la atmósfera estelar no afecten lo que ocurre en el interior. Generalmente esto es así en un excelente grado de aproximación. Los cálculos de evolución estelar son realizados usando un modelo de atmósfera muy simplificado. Solamente cuando las magnitudes absolutas y los índices de color son requeridos para llevar los resultados teóricos a distintos planos observacionales se consideran modelos detallados de atmósferas, aplicados a resultados evolutivos previamente obtenidos.

Sin embargo, en el caso de las enanas blancas, un tratamiento detallado de la atmósfera estelar puede afectar las predicciones evolutivas, particularmente en relación con las edades de enfriamiento. En este capítulo presentamos cálculos evolutivos de HeWDs obtenidos consistentemente con modelos detallados de atmósfera.

Los resultados de esta investigación han sido publicados en Serenelli, Althaus, Rohrmann \& Benvenuto (2001).

\subsection{Introducción}

Las HeWDs son WD de baja masa cuyos núcleos de helio no han alcanzado la masa necesaria para encender las reacciones nucleares que fusionen el helio. Es generalmente aceptado que la formación de las HeWDs en escalas temporales menores que la edad de la Vía Láctea puede ocurrir sólo si estos objetos son el resultado de episodios de pérdida de masa en sistemas binarios cercanos (ver Iben \& Livio, 1993 para un review del tema). Estas HeWDs pueblan la cola de baja masa $\left(M<0.4 \mathrm{M}_{\odot}\right)$ de la distribución de masas de las WDs (Bergeron, Saffer \& Liebert, 1992; Bragaglia, Renzini \& Bergeron, 1995; Saffer, Livio \& Yungelson, 1998).

El problema de la determinación de las edades de las HeWDs es un problema bien conocido en el estudio de estas estrellas. En efecto, las edades de enfriamiento de las HeWDs están íntimamente relacionadas con la masa de las envolturas de hidrógeno que las HeWDs tienen al entrar en el track final de enfriamiento. Este tema fue discutido en detalle en el capítulo 3 y no volveremos aquí sobre él. Remarquemos simplemente el hecho que cuando la difusión microscópica de los elementos es incluida en los cálculos evolutivos naturalmente surge un comportamiento cualitativamente diferenciado entre HeWDs con masas por debajo de $0.18 \mathrm{M}_{\odot} \mathrm{y}$ 
masas por encima de este valor. En efecto, los modelos más masivos sufren flashes termonucleares por efectos de la difusión y tienen posteriormente una evolución rápida en su rama de enfriamiento. Por el contrario, los modelos con masas menores al umbral mencionado, se enfrían lentamente debido a la energía nuclear liberada a través de la cadena protón-protón en la base de la envoltura de hidrógeno. En el capítulo 3 hemos mostrado que esto conduce de modo natural a una solución para la discrepancia entre las edades de HeWDs y púlsares de milisegundo componentes de sistemas binarios (ver también Althaus, Serenelli \& Benvenuto, 2001a,b, y Althaus, Serenelli \& Benvenuto, 2001c para un detalle sobre los flashes inducidos por la difusión). De acuerdo a estos cálculos, las HeWDs con masas mayores a $0.18 \mathrm{M}_{\odot}$ pueden alcanzar luminosidades muy bajas en tiempos menores a la edad del Universo y en conexión con estas predicciones la WD LSH 3250 es particularmente relevante. En efecto, esta estrella tiene una luminosidad superficial de $\log \mathrm{L} / \mathrm{L}_{\odot}=-4.57 \pm 0.04$ (Harris et al., 1999), lo cual la ubica entre las luminosidades más bajas encontradas para cualquier WD. Es probable que esta WD tenga un núcleo de helio y una $\mathrm{T}_{\text {eff }}$ muy baja (Harris et al., 1999) por lo que su espectro emergente estaría dominado por la absorción inducida por colisiones del hidrógeno molecular (CIA). Esta absorción molecular causa que las WDs frías se vuelvan más azules a medida que se enfrían (Hansen, 1998; Saumon \& Jacobson, 1999; Rohrmann, 2001). Secuencias evolutivas de WD basadas en cálculos detallados del transporte radiativo apropiados para WDs de carbono-oxígeno han sido presentados por Hansen $(1998,1999)$ y Salaris et al. (2000).

Si las HeWDs están caracterizadas por edades de enfriamiento cortas, como se presentó en el capítulo 3, entonces muchas de ellas presentarán colores azules como resultado de los efectos de la CIA del hidrógeno molecular. En vista de esto, es necesario rever las edades de las HeWDs frías y proveer además colores y magnitudes para estas estrellas de manera autoconsistente con las predicciones de la evolución estelar y la difusión de los elementos químicos. Los cálculos que se presentan aquí representan una mejora con respecto a los del capítulo 3 en lo que respecta a las edades de las HeWDs frías ya que incluyen un tratamiento apropiado para la atmósfera estelar, es decir para la condición externa del problema de evolución estelar, basado en un tratamiento no-gris de la misma (Rohrmann, 2001). Desde el punto de vista de las edades de las HeWDs frías, éste es un punto importante ya que el enfriamiento de las WDs es muy sensible al tratamiento de la atmósfera, en particular por la extensión que alcanza la convección en las envolturas de estas estrellas.

\subsection{Detalles computacionales}

Los cálculos evolutivos fueron realizados con el mismo código evolutivo usado en los cálculos presentados en el capítulo 3 y no repetiremos los detalles aquí. Dado que los efectos de un tratamiento detallado de la atmósfera son relevantes a $\mathrm{T}_{\text {eff }}$ bajas, hemos usado los mismos modelos de HeWDs que en el capítulo 3 y hemos recalculado la evolución de estos modelos una vez que entran en la etapa final de enfriamiento. Las masas de los modelos de HeWD presentados son, entonces, 0.406, 0.360, 0.327, 0.292, 0.242, 0.196 y $0.169 \mathrm{M}_{\odot}$ y las edades características en la rama de enfriamiento estarán determinadas por la ocurrencia o no de flashes termonucleares por efectos de la difusión.

El CEELP ha sido acoplado con un código de atmósferas no-gris, desarrollado por el Dr. René Rohrmann. Este código ha sido adaptado para tratar mezclas de hidrógeno y helio, características de las atmósferas de las HeWDs. Presentamos aquí una descripción breve de las principales características del mismo y referimos al lector a Rohrmann (2001) para una 
descripción exhaustiva del mismo y una comparación con otros cálculos atmosféricos relevantes existentes en la literatura.

El código de atmósferas está construido bajo la hipótesis de gravedad constante, equilibrio termodinámico local y geometría plano-paralela, e incluye hidrógeno y helio (metalicidad cero). El transporte de energía por radiación y convección son tenidas en cuenta y las ecuaciones resultantes son resueltas mediante una técnica de linealización estándar. La física constitutiva está basada en una ecuación de estado ideal. Las especies que han sido consideradas son: $\mathrm{H}$, $\mathrm{H}_{2}, \mathrm{e}^{-}, \mathrm{H}^{-}, \mathrm{H}_{2}^{+}, \mathrm{H}_{3}^{+}, \mathrm{He}, \mathrm{He}^{+}$y $\mathrm{He}^{++}$. Aunque las abundancias de $\mathrm{H}_{2}^{+} \mathrm{y} \mathrm{H}_{3}^{+}$son generalmente despreciables, su presencia afecta la absorción y emisión de la radiación significativamente (Saumon et al., 1994). Las funciones de partición para el $\mathrm{H}, \mathrm{H}_{2}, \mathrm{H}_{2}^{+}, \mathrm{H}_{3}^{+}$son las de Irwin (1981); Sauval \& Tatum (1984) y Neale \& Tennyson (1995) respectivamente.

Todos los procesos de scattering, ligado-libre y libre-libre relevantes que contribuyen a la opacidad han sido incluidos en los cálculos. A bajas $\mathrm{T}_{\text {eff }}$ la opacidad CIA del hidrógeno molecular debida a colisiones con $\mathrm{H}_{2}$ y $\mathrm{He}$ es una fuente de opacidad muy importante en el infrarrojo y domina la forma del espectro emergente. Aquí se han incluido los cálculos de las secciones eficaces de CIA para $\mathrm{H}_{2}-\mathrm{H}_{2}$ y $\mathrm{H}_{2}$-He de Borysow, Jorgensen \& Zheng (1997). La convección es tratada dentro del formalismo de la mixing length theory (MLT2) y ha sido incluida de manera auto-consistente en la ecuación de conservación de la energía y en el procedimiento de linealización. Vale la pena mencionar que por debajo de $\mathrm{T}_{\text {eff }}=8000 \mathrm{~K}$, el perfil de temperatura se vuelve insensible a la parametrización de la teoría de la MLT (Bergeron, Wesemael \& Fontaine, 1992). Los índices de color de banda ancha han sido calculados usando los pasabandas ópticos e infrarrojos de Bessell \& Brett (1988) y Bessell (1990) respectivamente, con constantes de calibración tomadas de Bergeron, Ruiz \& Leggett (1997).

Los valores de presión y temperatura necesarios para integrar las ecuaciones de envoltura están dados a una profundidad óptica $\tau \approx 25$. En este punto la aproximación de difusión para la transferencia radiativa puede suponerse válida y las ecuaciones de estructura estelar en la envoltura pueden integrarse usando las opacidades medias de Rosseland.

\subsection{Resultados}

Comenzamos examinando las edades evolutivas para algunas masas estelares seleccionadas. Los resultados se muestran en la figura 4.1 en la cual se grafica la $T_{\text {eff }}$ contra la edad junto con los datos observacionales para las HeWD compañeras de los púlsares de milisegundo PSR J1012+5307 y PSR B1855+09 (Callanan, Garnavich, Koester, 1998; van Kerkwijk et al., 2000, respectivamente). Sólo se incluyen los resultados de la evolución correspondiente a la rama final de enfriamiento. Para masas mayores $\mathrm{a} \approx 0.18 \mathrm{M}_{\odot}$ los modelos experimentan flashes de hidrógeno inducidos por la difusión y tienen envolturas de hidrógeno delgadas y muy poca liberación de energía nuclear durante la rama de enfriamiento. Esto trae como consecuencia que las edades de enfriamiento son marcadamente menores con respecto a los modelos con masas menores que el límite mencionado, ya que estos modelos no sufren flashes termonucleares (ver capítulo 3).

Notar que las diferencias en el enfriamiento de las HeWDs tiene su origen en el tratamiento detallado del modelo atmosférico. La inclusión de condiciones externas apropiadas puede disminuir las edades para una $\mathrm{T}_{\text {eff }}$ dada hasta en 1 Gyr en el rango $4000 \leq \mathrm{T}_{\text {eff }} \leq 5500 \mathrm{~K}$. Durante esta fase evolutiva, la temperatura central está fuertemente ligada con la estratificación de la temperatura en las capas externas. Cuando la convección alcanza la región donde la degen- 


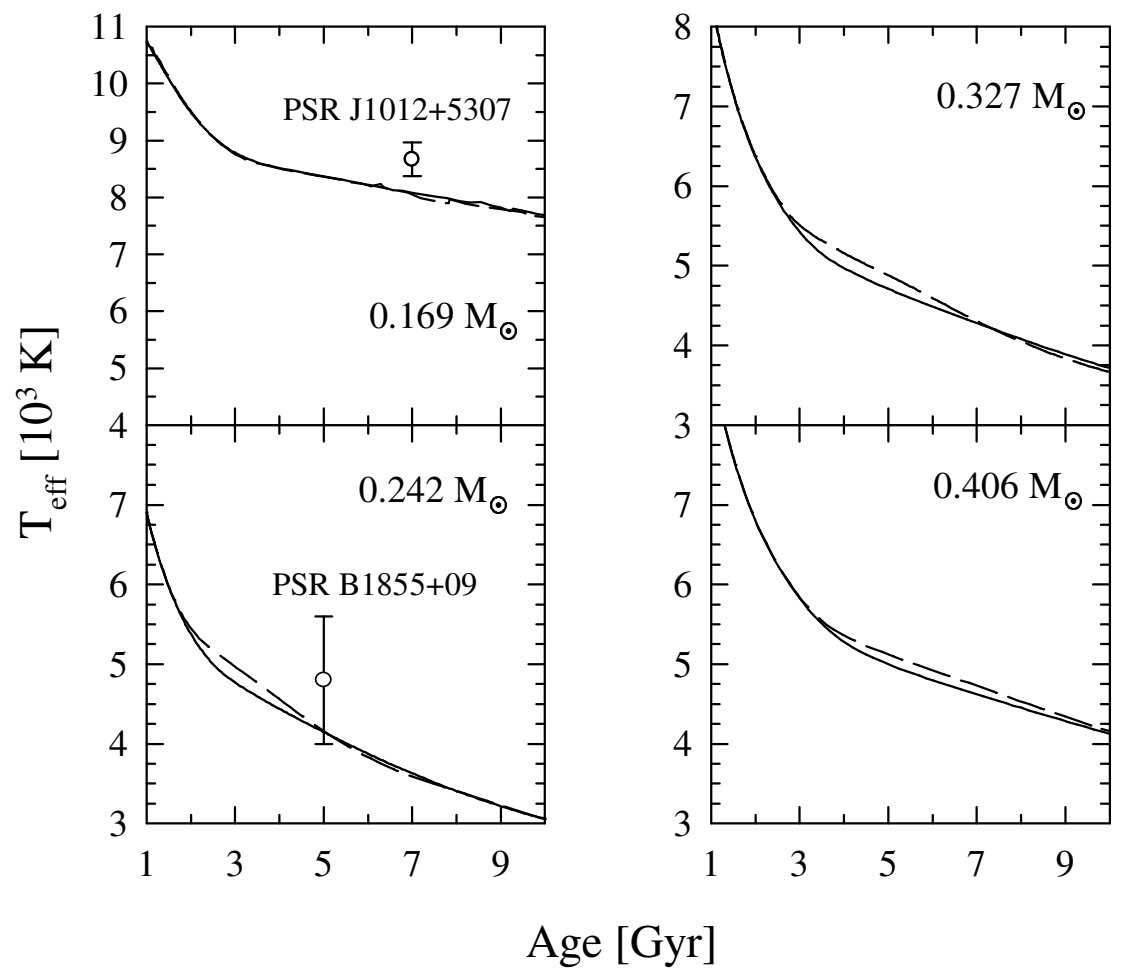

Figura 4.1: $\mathrm{T}_{\text {eff }}$ como función de la edad para algunas masas estelares seleccionadas. Las líneas sólidas corresponden al caso de atmósfera no-gris y las líneas de rayas al caso simplificado de una atmósfera gris. Ambos conjuntos de cálculos incluyen la difusión de los elementos. Notar que para los modelos más masivos y $4000 \leq \mathrm{T}_{\text {eff }} \leq 5500 \mathrm{~K}$, las edades de enfriamiento se vuelven menores en el caso del tratamiento no-gris de la atmósfera.

eración es importante, la temperatura central cae sustancialmente (el transporte convectivo de la energía pone en "contacto térmico" el interior estelar con las regiones externas mucho más eficientemente que el transporte radiativo), y la estrella tiene un exceso inicial de energía interna que debe ser radiado, dando lugar a un enlentecimiento de la evolución durante esta fase evolutiva, como queda evidenciado por el cambio de pendiente de las curvas de enfriamiento que se muestran en la figura 4.1. Durante los estados avanzados de la evolución, la estratificación térmica de la envoltura está afectada por el uso de atmósferas no-grises de manera tal que la profundidad máxima alcanzada por la base de la zona convectiva es marcadamente menor que en el caso de atmósfera gris (ver también Bergeron, Ruiz \& Leggett, 1997). Esto puede verse en la figura 4.2, donde se muestra la evolución de la base de la zona convectiva externa en términos de $\mathrm{T}_{\text {eff }}$ para el modelo de $0.242 \mathrm{M}_{\odot}$. Cuando $\mathrm{T}_{\text {eff }}$ decrece por debajo de $7000 \mathrm{~K}$, el tratamiento no-gris predice una zona convectiva externa menos profunda (este comportamiento es el mismo independientemente de la masa del modelo). Como resultado, la convección alcanza el núcleo degenerada a $\mathrm{T}_{\text {eff }}$ más bajas en los modelos con atmósferas no-grises y por lo tanto la caída en la temperatura central se produce más tarde que en los modelos grises, como se puede ver en la figura 4.3. Este comportamiento explica las características de las curvas de enfriamiento de ambos conjuntos de cálculos.

Otra característica interesante relacionada con la extensión de la zona convectiva externa es la siguiente. Como se mencionó en el capítulo 3, la envoltura de hidrógeno que queda después 


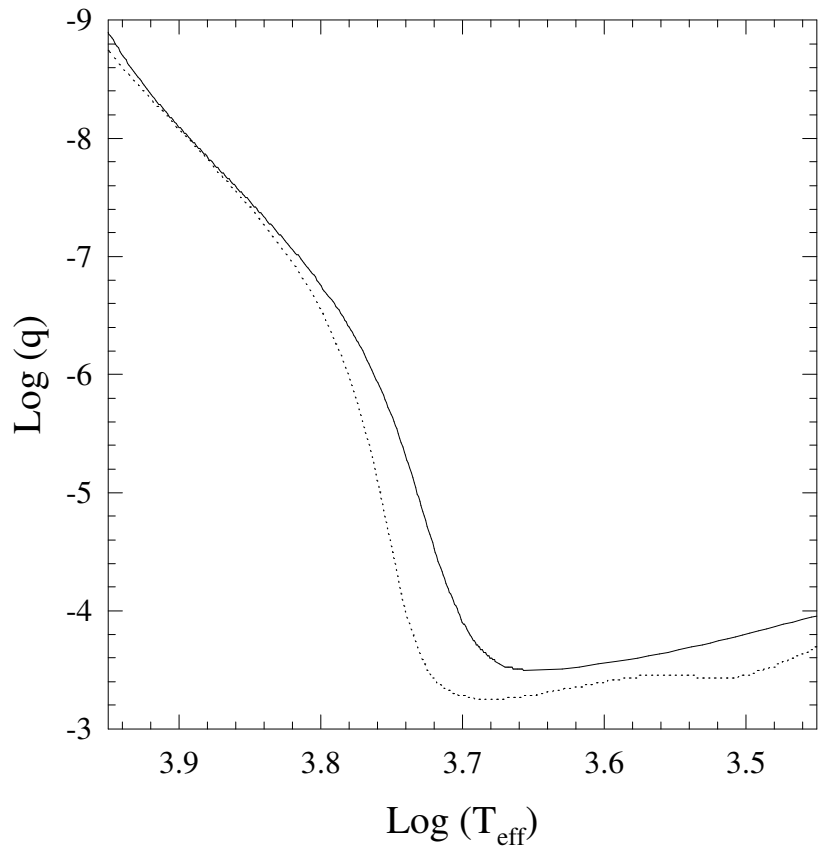

Figura 4.2: Posición de la base de la envoltura convectiva en términos de la fracción externa de masa $q$ en función de la temperatura para el modelo de $0.242 \mathrm{M}_{\odot}$. Las líneas sólidas y punteadas denotan los modelos con atmósfera no-gris y gris respectivamente. Notar que la zona convectiva externa es menos profunda en el caso de los modelos con tratamiento detallado de la atmósfera.

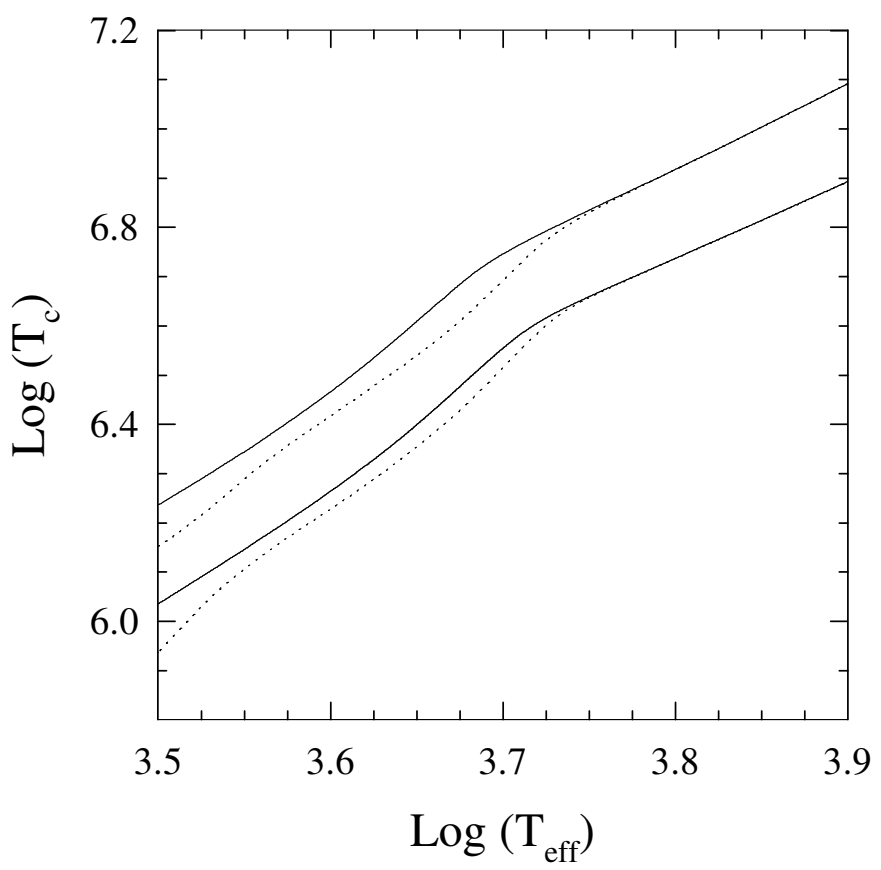

Figura 4.3: Temperatura central como función de la $\mathrm{T}_{\text {eff }}$ para los modelos de 0.242 y $0.406 \mathrm{M}_{\odot}$ (curvas superiores e inferiores respectivamente). Las líneas sólidas y punteadas son como en la figura 4.2. 


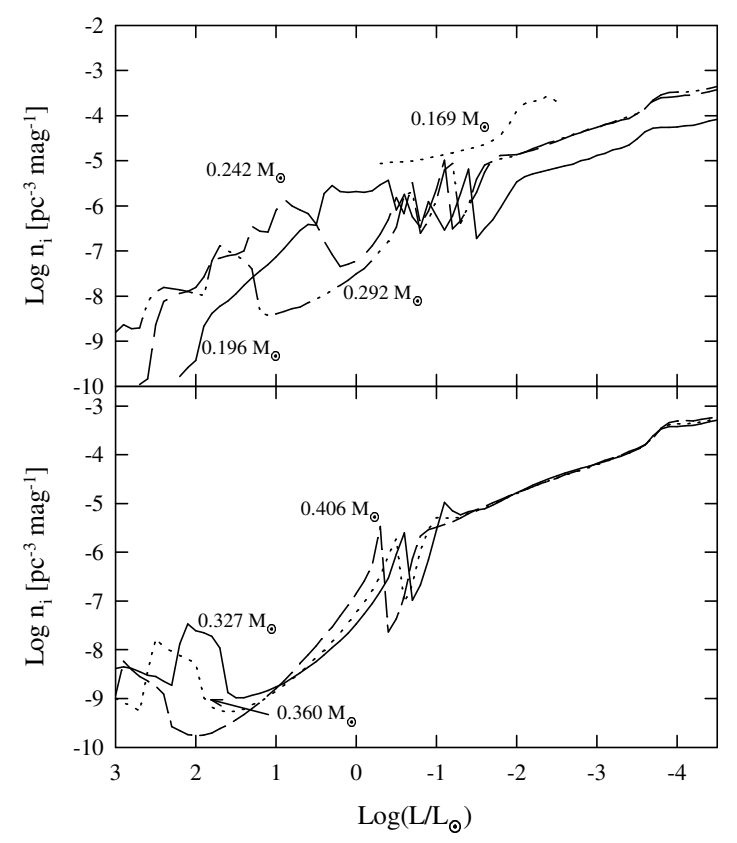

Figura 4.4: Función de luminosidad individual contra luminosidad superficial para nuestros modelos de HeWD. Los picos a luminosidades intermedias corresponden a las fases evolutivas antes de la ocurrencia de los flashes, y las protuberancias a luminosidades altas reflejan la lenta evolución que antecede la entrada de los modelos a la rama de enfriamiento. Los modelos menos masivos experimentan varios flashes, por eso alcanzan la parte azul del HRD varias veces, de modo que sus funciones de luminosidad presenta protuberancias más extendidas.

de los flashes de hidrógeno en los modelos de HeWDs puede ser lo suficientemente delgada como para que la convección sea capaz de mezclar esta envoltura con regiones más internas, ricas en hidrógeno. De esta manera, para algunas masas estelares, los modelos de HeWDs están caracterizados por envolturas mixtas de hidrógeno y helio para $\mathrm{T}_{\text {eff }}$ alrededor de $5000 \mathrm{~K}$. Sin embargo, dado que un tratamiento detallado de la atmósfera conduce a zonas convectivas externas menos profundas, en los presentes cálculos encontramos que la convección no penetra lo suficiente en el interior estelar como para que la abundancia de helio transportada hacia la superficie sea significativa, por lo que las envolturas están caracterizadas siempre por una composición química de hidrógeno casi puro.

Partiendo de las curvas de enfriamiento, pueden realizarse predicciones acerca del número de HeWDs por unidad de volumen como función de su luminosidad. Para este fin, hemos transformado las curvas de enfriamiento de nuestro conjunto de modelos en la función de luminosidad total de acuerdo a la relación dada por:

$$
\Phi=\sum_{i} n_{i}
$$

con

$$
n_{i}=\frac{k_{i} \Delta t}{V}
$$

Aquí, $k_{i}$ es la tasa de nacimiento de HeWDs para una masa estelar dada, la suma de las cuales está normalizada a la tasa de naciemiento total a la cual las HeWDs son producidas $\left(0.14 \mathrm{yr}^{-1}\right.$; 


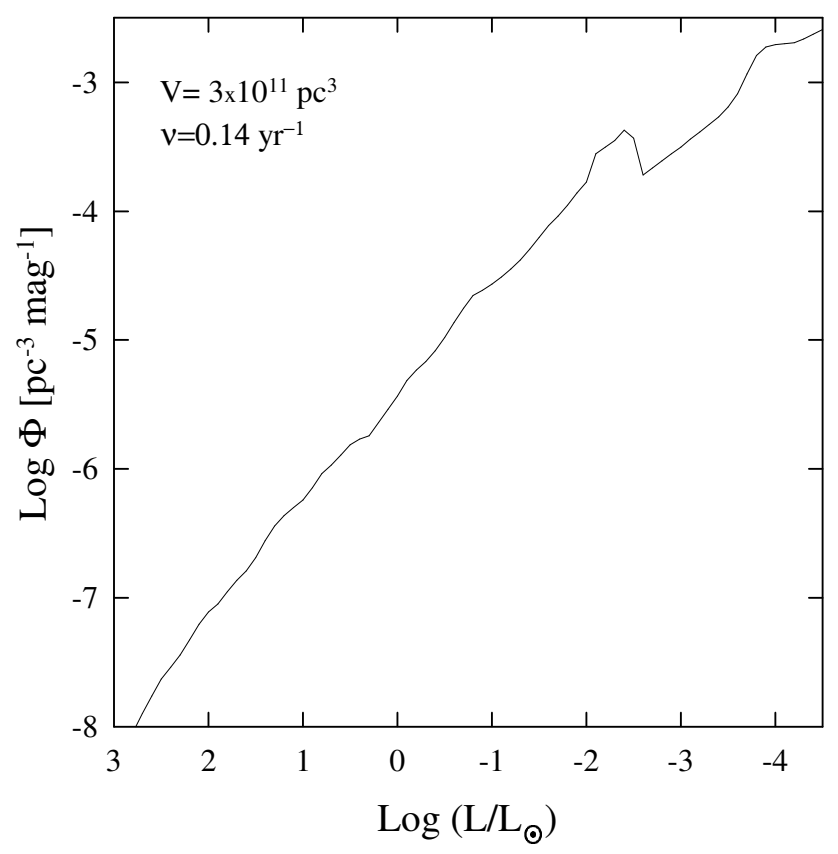

Figura 4.5: Función de luminosidad individual contra luminosidad superficial para nuestros modelos de HeWD. Los picos a luminosidades intermedias corresponden a las fases evolutivas antes de la ocurrencia de los flashes, y las protuberancias a luminosidades altas reflejan la lenta evolución que antecede la entrada de los modelos a la rama de enfriamiento. Los modelos modelos menos masivos experimentan varios flashes, por eso alcanzan la parte azul del HRD varias veces, de modo que sus funciones de luminosidad presenta protuberancias más extendidas.

ver Iben, Tutukov \& Yungelson, 1997), $n_{i}$ es el número de HeWDs por unidad de volumen de una masa dada en el intervalo de magnitudes $M_{b}-0.5$ a $M_{b}+0.5$ y $V$ es el volumen del disco galáctico $\left(3 \times 10^{11} \mathrm{pc}^{3}\right)$. En base a estas suposiciones, la funciones de luminosidad obtenidas para cada una de las masas de nuestros modelos se muestran en la figura 4.4. Una característica notable son los picos que pueden observarse en luminosidades intermedias, que corresponden a las fases de evolución lenta de las fases inmediatamente anteriores a la ocurrencia de los flashes termonucleares. A luminosidades altas, la distribución teórica de cada HeWD exhibe una protuberancia. Estas protuberancias son consecuencia del rate de evolución lento previo a que la estrella entre en la rama de enfriamiento. Para entender esto, debe tenerse en mente que como resultado de los flashes termonucleares, los modelos alcanzan altas luminosidades superficiales más de una vez durante su evolución. Los modelos menos masivos experimentan varios episodios de flash, por lo tanto las funciones de luminosidad resultantes muestran protuberancias más extendidas.

La función de luminosidad total, $\Phi$, resulta de superponer las funciones de luminosidad individuales y se muestra en la figura 4.5. Notar que durante la mayor parte del rango de luminosidades mostrado la función de luminosidad exhibe un comportamiento monótonamente creciente hasta $\log \mathrm{L} / \mathrm{L}_{\odot} \approx-2.5$. Casi nada del comportamiento de cada una de las funciones de luminosidad individuales permanece. Esto es esperado, ya que para diferentes masas estelares los flashes ocurren a diferentes luminosidades superficiales, los picos observados en la figura 4.4 se compensan entre sí. A $\log \mathrm{L} / \mathrm{L}_{\odot} \approx-2.5$, la función de luminosidad muestra un mínimo local 


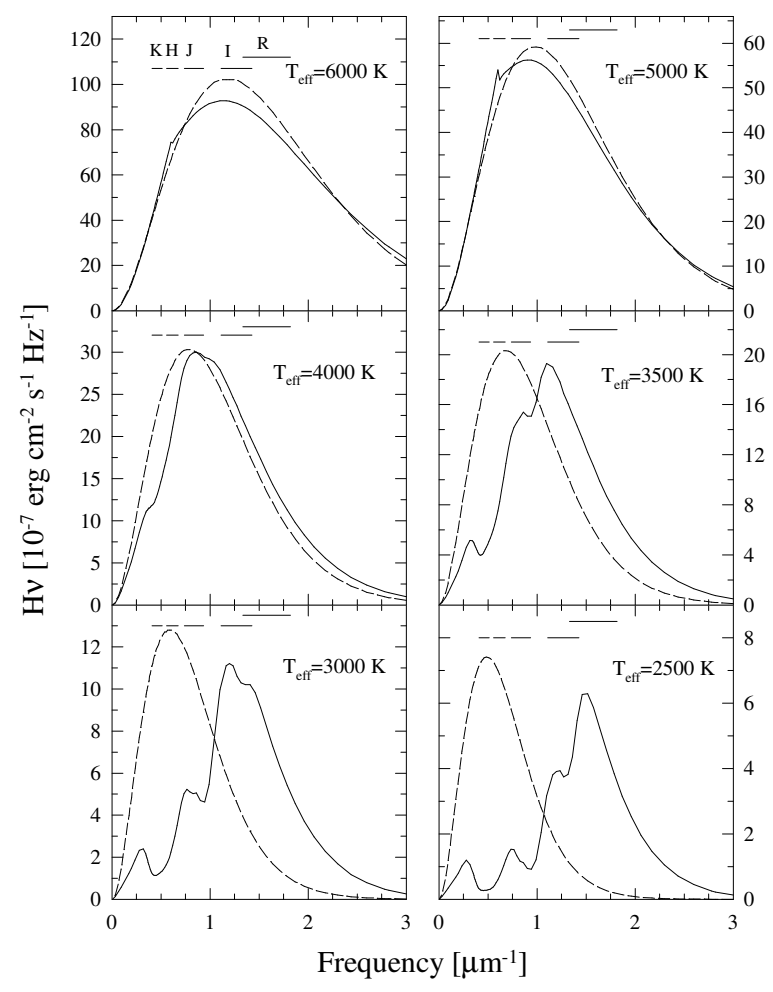

Figura 4.6: Espectro emergente para el modelo de $0.292 \mathrm{M}_{\odot}$ a valores seleccionados de $\mathrm{T}_{\text {eff }}$. Las líneas sólidas muestran los resultados del tratamiento detallado de la atmósfera, mientras que las líneas rayadas muestran los resultados para el cuerpo negro. Se indican también los rangos de frecuencia aproximados que cubren algunos filtros infrarrojos. Notar que a medida que la $\mathrm{T}_{\text {eff }}$ disminuye, el espectro se vuelve más azul. Esto es consecuencia directa de la opacidad CIA, que es dominante a bajas $\mathrm{T}_{\text {eff }}$.

como resultado de la contribución de los modelos que no experimentan flashes de hidrógeno. En efecto, los modelos que no sufren flashes están caracterizados por una evolución muy lenta en la rama de enfriamiento. En particular, el modelo de $0.169 \mathrm{M}_{\odot}$ tiene una edad de 14 Gyr a $\log \mathrm{L} / \mathrm{L}_{\odot}=-2.5$, por lo que se espera que no contribuya a la población de enanas blancas a luminosidades más bajas en una escala temporal igual a la edad del Universo. A $\log \mathrm{L} / \mathrm{L}_{\odot} \approx-4$, la función de luminosidad muestra una protuberancia pequeña. Como se mencionó antes, cuando la convección alcanza el borde externo del núcleo convectivo hay un exceso inicial de energía del cual la estrella debe deshacerse por lo que el proceso de enfriamiento se enlentece, produciendo esa protuberancia en la función de luminosidad. Finalmente, nótese que para construir $\Phi$ hemos considerado las HeWDs como estrellas aisladas, es decir hemos despreciado la posible existencia de fusiones entre estrellas componentes de sistemas binarios cercanos debido a la radiación de ondas gravitatorias. De acuerdo con esto, se debe ser cuidadoso al comparar estos resultados con las obsrvaciones.

En la figura 4.6 se muestra el flujo emergente para nuestro modelo de $0.292 \mathrm{M}_{\odot}$ en ciertos valores de $\mathrm{T}_{\text {eff }}$. Los resultados del modelo no-gris de atmósfera (línea sólida) son comparados con las predicciones del modelo de cuerpo negro (línea rayada), a la misma $\mathrm{T}_{\text {eff }}$. Es importante tener en cuenta que cuando los modelos alcanzan las $\mathrm{T}_{\text {eff }}$ mostradas en la figura 4.6, y como consecuencia de la sedimentación gravitatoria, las envolturas de las HeWDs son de hidrógeno 


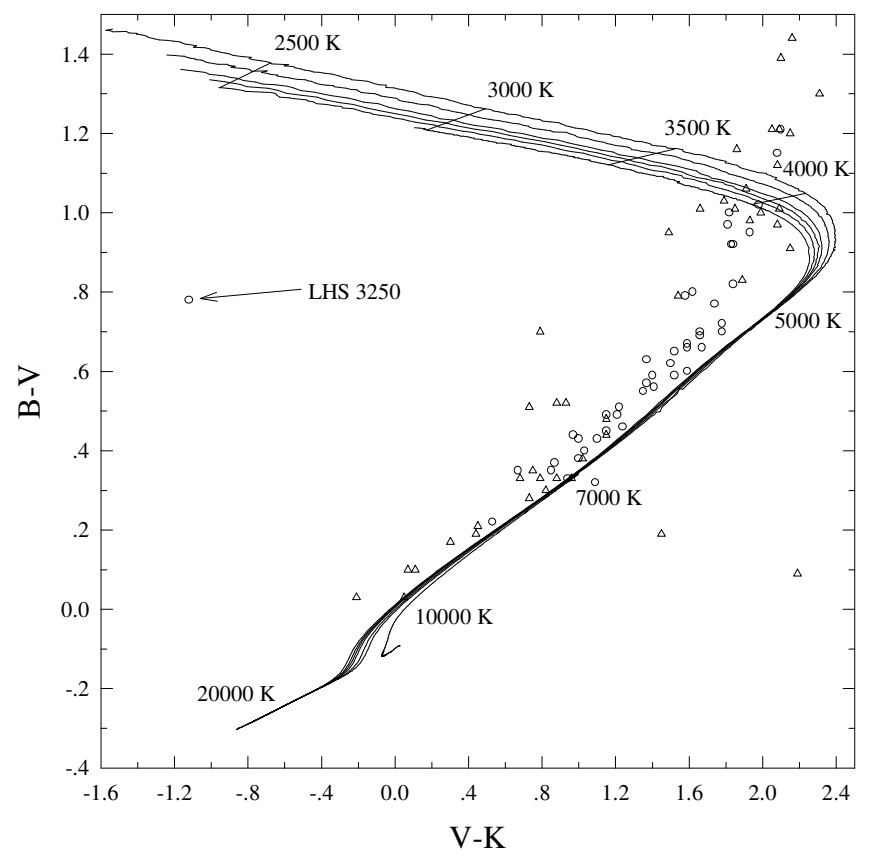

Figura 4.7: Diagrama color-color $(B-V, V-K)$ para nuestros modelos de HeWD de 0.196, 0.242, $0.292,0.327,0.360,0.406 \mathrm{M}_{\odot}$. Los puntos de igual $\mathrm{T}_{\text {eff }}$ están representados por líneas delgadas. Las muestras observacionales para estrellas WD DA frías (círculos abiertos) y no-DA (triángulos abiertos) de Bergeron, Leggett \& Ruiz (2001) están también inluidas, así como también la ubicación de la WD LHS 3250 de acuerdo a Harris et al. (1999).

puro. Para valores de $\mathrm{T}_{\text {eff }}$ altos, la opacidad por $\mathrm{H}^{-}$es dominante. Ya que esta opacidad es casi independiente de la frecuencia, el flujo emergente es muy similar al del cuerpo negro. Cuando la $\mathrm{T}_{\text {eff }}$ cae por debajo de $4000 \mathrm{~K}$, sin embargo, puede observarse que el espectro emergente se torna más azul. Este efecto, reportado también por Saumon et al. (1994) y más recientemente por Hansen (1998, 1999); Salaris et al. (2000) y Rohrmann (2001) (principalemte en el contexto de enanas blancas de carbono-oxígeno) es debido a la opacidad CIA del hidrógeno molecular, que reduce el flujo infrarrojo y fuerza a la radiación a emerger a frecuencias mayores. Como resultado, a medida que las HeWDs envejecen se vuelven más azules. Este efecto, por supuesto, afecta los diagramas color-color y color-magnitud. Los índices de color que involucran una banda en el infrarrojo muestran un turn-off pronunciado a bajas $\mathrm{T}_{\text {eff }} \mathrm{y}$ se vuelven más azules después de alcanzar un máximo. Esto queda bien ilustrado en la figura 4.7, en la cual se muestra el diagrama $(B-V, V-K)$ para todos nuestros modelos de HeWD junto con los datos observacionales para WDs DA y no-DA de acuerdo a Bergeron, Leggett \& Ruiz (2001). La WD peculiar LHS 3250 también se muestra en el gráfico, de acuerdo a la determinación de Harris et al. (1999). Notar que el color $V-K$ se vuelve marcadamente azul por debajo de $\mathrm{T}_{\text {eff }}=5000 \mathrm{~K}$ en este diagrama.

Analicemos ahora la evolución de nuestros modelos en los diagramas color-magnitud. En las figuras 4.8 y 4.9 se muestra el comportamiento de la magnitud visual $M_{V}$ de nuestros modelos en función de los índices de color $V-I$ y $B-V$ respectivamente. Se incluyen las mismas muestras observacionales que en la figura 4.7. Para el modelo de $0.169 \mathrm{M}_{\odot}$ sólo se muestran las fases de bajas $M_{V}$ debido a que para evolucionar a luminosidades menores este 


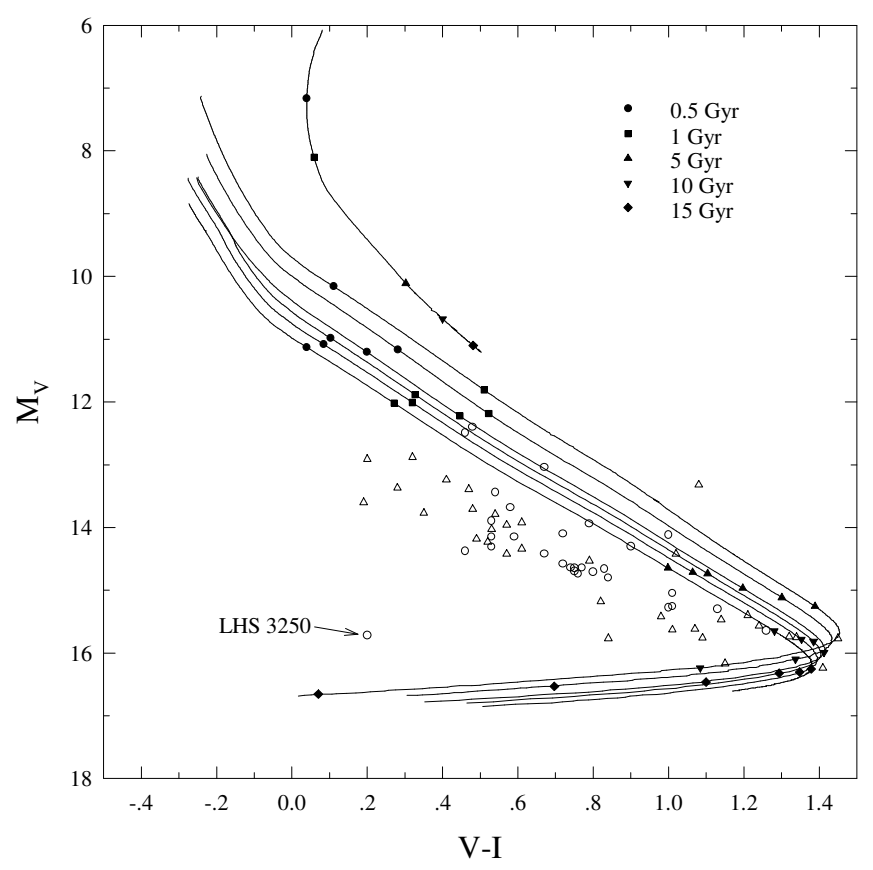

Figura 4.8: Magnitud absoluta visual $M_{V}$ en términos del índice de color $V-I$ para nuestros modelos de HeWD (masa creciente desde arriba hacia abajo). En cada curva, los símbolos llenos indican edades de enfriamiento. Se incluyen los mismos datos observacionales que en la figura 4.7. Los modelos con masas mayores a $0.196 \mathrm{M}_{\odot}$ exhiben un turn-off pronunciado en etapas avanzadas de su evolución y se vuelven más azules con la evolución subsiguiente. De acuerdo con nuestros cálculos, HeWDs con masas entre 0.18 y $0.30 \mathrm{M}_{\odot}$ podrían evolucionar más allá del turn-off en tiempos menores a $15 \mathrm{Gyr}$.

modelo requiere edades excesivamente grandes. En contraste, los modelos más masivos pueden alcanzar magnitudes grandes en edades menores a un tiempo de Hubble. En efecto, como puede verse en la figura 4.8, los modelos de HeWD en el rango de masas entre 0.18 y $0.30 \mathrm{M}_{\odot}$ alcanzan el punto de turn-off y se vuelven azules en edades menores a 15 Gyr. Específicamente, estos modelos tienen entre 6 y 9 Gyr de edad en el turn-off, que ocurre a $M_{V} \approx 16$. Notar que durante su evolución subsiguiente, permanecen más brillantes que $M_{V} \approx 16.5$. Los resultados presentados aquí muestran que es posible que muchas HeWDs pueden haber tenido tiempo suficiente para evolucionar más allá del punto de turn-off y presentar colores azules. Las HeWDs más masivas presentan un comportamiento similar en los diagramas color-magnitud, pero las edades involucradas son mayores. Por ejemplo, el modelo de $0.406 \mathrm{M}_{\odot}$ necesita alrededor de 13 Gyr para alcanzar el punto de turn-off en el índice de color $V-I$. Es importante remarcar nuevamente que el hecho que las HeWDs puedan alcanzar el turn-off en edades menores a la del Universo es consecuencia de los efectos de la difusión en la evolución de las HeWDs. Modelos de HeWD sin difusión no alcanzan el punto de turn-off ni siquiera en alrededor de 20 Gyr. El diagrama color-magnitud para el índice $B-V$ (figura 4.9) no muestra un turn-off a bajas $\mathrm{T}_{\text {eff }}$. Hay que mencionar que a altas $\mathrm{T}_{\text {eff }}$, nuestras predicciones para el índice $B-V$ muestran discrepancias con respecto a los presentados por Bergeron, Wesemael \& Beauchamp (1995) porque nuestros cálculos no consideran los efectos de las opacidades por ensanchamiento de líneas.

Finalmente, en la tabla 4.1 listamos los índices de color para los modelos de 0.169, 0.242, 


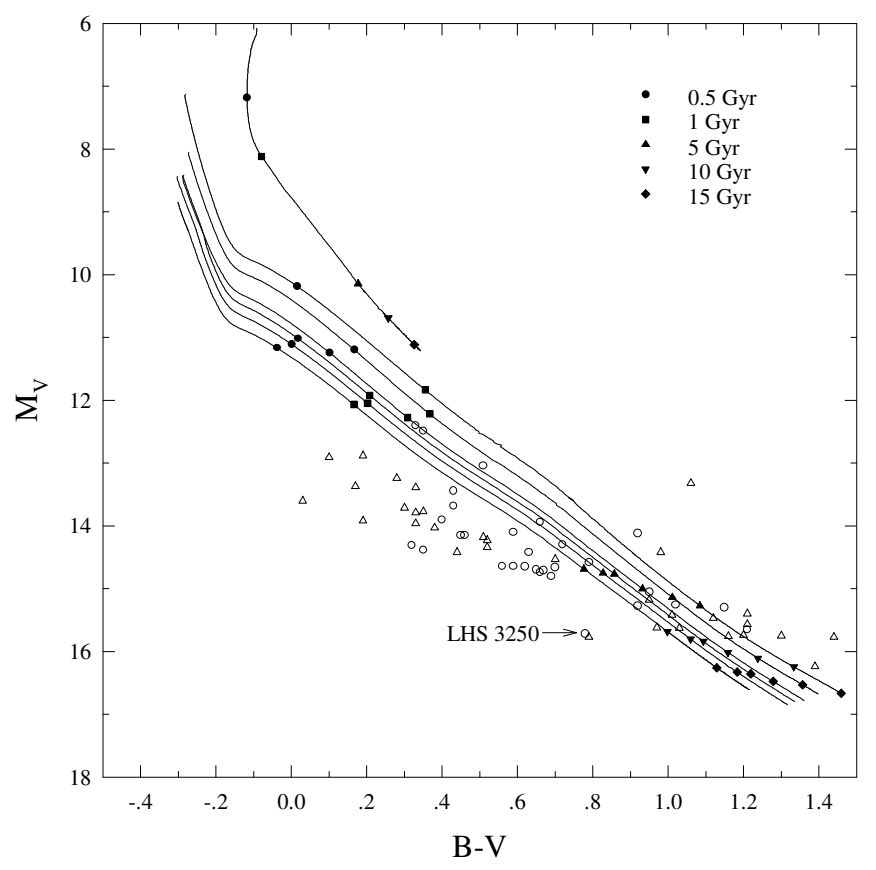

Figura 4.9: Igual que la figura 4.8 pero para el índice de color $B-V$. Ya que en este caso ninguna banda cae en la región espectral afectada por las opacidad CIA, el índice $B-V$ no muestra un turn-off a bajas $\mathrm{T}_{\mathrm{eff}}$.

0.327 y $0.406 \mathrm{M}_{\odot}$ para algunos valores seleccionados de $\mathrm{T}_{\text {eff }}$. Además, para cada masa estelar se muestran la gravedad superficial, la edad, la magnitud absoluta visual y la corrección bolométrica. Esta última se calcula de acuerdo a (Bergeron, Saumon \& Wesemael, 1995)

$$
B C=2.5 \log \int_{0}^{\infty} H_{\lambda} S_{\lambda}^{V} d \lambda-10 \log T_{\text {eff }}+15.6165
$$

donde $H_{\lambda}$ y $S_{\lambda}^{V}$ son el flujo emergente monocromático a la temperatura efectiva $\mathrm{T}_{\text {eff }}$ y la función de transmisión del filtro $V$, respectivamente.

Table 4.1: Modelos seleccionados para las secuencias de $0.169,0.242,0.327$ y $0.406 \mathrm{M}_{\odot}$.

\begin{tabular}{cccccccccccc}
\hline$M_{*} / \mathrm{M}_{\odot}$ & $\mathrm{T}_{\text {eff }}$ & $\log (g)$ & $\operatorname{Edad}(\mathrm{Gyr})$ & $\mathrm{B}-\mathrm{V}$ & $\mathrm{V}-\mathrm{R}$ & $\mathrm{V}-\mathrm{K}$ & $\mathrm{R}-\mathrm{I}$ & $\mathrm{J}-\mathrm{H}$ & $\mathrm{H}-\mathrm{K}$ & $\mathrm{BC}$ & $\mathrm{M}_{V}$ \\
\hline & & & & & & & & & & & \\
0.169 & 10000 & 6.0621 & 1.56 & -0.01 & 0.04 & 0.03 & 0.06 & 0.02 & -0.06 & -0.34 & 8.70 \\
0.169 & 9500 & 6.1624 & 1.99 & 0.04 & 0.07 & 0.15 & 0.08 & 0.04 & -0.06 & -0.29 & 9.11 \\
0.169 & 9000 & 6.2555 & 2.60 & 0.10 & 0.11 & 0.30 & 0.11 & 0.07 & -0.05 & -0.25 & 9.55 \\
0.169 & 8500 & 6.3499 & 4.11 & 0.16 & 0.14 & 0.46 & 0.14 & 0.10 & -0.04 & -0.23 & 10.01 \\
0.169 & 8000 & 6.4256 & 7.63 & 0.22 & 0.18 & 0.64 & 0.18 & 0.14 & -0.02 & -0.21 & 10.44 \\
0.169 & 7500 & 6.4796 & 11.45 & 0.28 & 0.22 & 0.82 & 0.22 & 0.18 & -0.01 & -0.19 & 10.84 \\
0.169 & 7050 & 6.5265 & 16.52 & 0.34 & 0.25 & 1.00 & 0.25 & 0.21 & 0.00 & -0.17 & 11.21
\end{tabular}




\begin{tabular}{|c|c|c|c|c|c|c|c|c|c|c|c|}
\hline$\overline{M_{*} / \mathrm{M}_{\odot}}$ & $\mathrm{T}_{\mathrm{eff}}$ & $\log g$ & Edad (Gyr) & $\mathrm{B}-\mathrm{V}$ & $\overline{V-R}$ & $\mathrm{~V}-\mathrm{K}$ & R-I & $\mathrm{J}-\mathrm{H}$ & H-K & $\mathrm{BC}$ & $\mathrm{M}_{V}$ \\
\hline 0.242 & 10000 & 6.9458 & 0.32 & 0.03 & 0.07 & 0.08 & 0.07 & 0.03 & -0.06 & -0.37 & 10.54 \\
\hline 0.242 & 9500 & 6.9616 & 0.37 & 0.08 & 0.09 & 0.20 & 0.09 & 0.05 & -0.05 & -0.32 & 10.76 \\
\hline 0.242 & 9000 & 6.9780 & 0.44 & 0.13 & 0.12 & 0.34 & 0.12 & 0.08 & -0.04 & -0.28 & 11.00 \\
\hline 0.242 & 8500 & 6.9945 & 0.53 & 0.18 & 0.15 & 0.49 & 0.15 & 0.11 & -0.03 & -0.25 & 11.26 \\
\hline 0.242 & 8000 & 7.0119 & 0.64 & 0.23 & 0.19 & 0.65 & 0.18 & 0.14 & -0.02 & -0.22 & 11.53 \\
\hline 0.242 & 7500 & 7.0291 & 0.78 & 0.29 & 0.22 & 0.83 & 0.22 & 0.18 & -0.01 & -0.20 & 11.83 \\
\hline 0.242 & 7000 & 7.0464 & 0.96 & 0.35 & 0.26 & 1.02 & 0.26 & 0.22 & 0.01 & -0.18 & 12.15 \\
\hline 0.242 & 6500 & 7.0637 & 1.19 & 0.43 & 0.30 & 1.23 & 0.30 & 0.26 & 0.03 & -0.16 & 12.50 \\
\hline 0.242 & 6000 & 7.0818 & 1.48 & 0.52 & 0.35 & 1.48 & 0.36 & 0.30 & 0.06 & -0.17 & 12.90 \\
\hline 0.242 & 5500 & 7.1044 & 1.88 & 0.64 & 0.42 & 1.76 & 0.42 & 0.34 & 0.09 & -0.21 & 13.38 \\
\hline 0.242 & 5000 & 7.1423 & 2.50 & 0.77 & 0.50 & 2.11 & 0.50 & 0.39 & 0.13 & -0.31 & 13.99 \\
\hline 0.242 & 4500 & 7.1779 & 3.80 & 0.91 & 0.59 & 2.36 & 0.59 & 0.38 & 0.09 & -0.45 & 14.68 \\
\hline 0.242 & 4000 & 7.1974 & 5.54 & 1.04 & 0.67 & 2.15 & 0.67 & 0.10 & -0.01 & -0.51 & 15.29 \\
\hline 0.242 & 3500 & 7.2082 & 7.57 & 1.15 & 0.73 & 1.42 & 0.70 & -0.18 & -0.19 & -0.37 & 15.76 \\
\hline 0.242 & 3000 & 7.2149 & 10.38 & 1.25 & 0.74 & 0.39 & 0.56 & -0.30 & -0.32 & -0.07 & 16.14 \\
\hline 0.242 & 2500 & 7.2207 & 15.20 & 1.36 & 0.65 & -0.79 & 0.00 & -0.21 & -0.48 & 0.34 & 16.54 \\
\hline 0.327 & 10000 & 7.3138 & 0.57 & 0.05 & 0.07 & 0.10 & 0.07 & 0.03 & -0.06 & -0.38 & 11.15 \\
\hline 0.327 & 9500 & 7.3240 & 0.68 & 0.09 & 0.10 & 0.22 & 0.09 & 0.05 & -0.05 & -0.34 & 11.35 \\
\hline 0.327 & 9000 & 7.3339 & 0.80 & 0.14 & 0.13 & 0.36 & 0.12 & 0.08 & -0.04 & -0.30 & 11.57 \\
\hline 0.327 & 8500 & 7.3433 & 0.94 & 0.19 & 0.16 & 0.50 & 0.15 & 0.11 & -0.03 & -0.26 & 11.81 \\
\hline 0.327 & 8000 & 7.3524 & 1.12 & 0.24 & 0.19 & 0.66 & 0.18 & 0.14 & -0.02 & -0.23 & 12.07 \\
\hline 0.327 & 7500 & 7.3613 & 1.33 & 0.29 & 0.22 & 0.83 & 0.22 & 0.18 & -0.01 & -0.20 & 12.34 \\
\hline 0.327 & 7000 & 7.3702 & 1.58 & 0.36 & 0.26 & 1.02 & 0.26 & 0.22 & 0.01 & -0.18 & 12.63 \\
\hline 0.327 & 6500 & 7.3794 & 1.90 & 0.43 & 0.30 & 1.23 & 0.30 & 0.26 & 0.03 & -0.16 & 12.96 \\
\hline 0.327 & 6000 & 7.3899 & 2.31 & 0.53 & 0.36 & 1.47 & 0.36 & 0.29 & 0.06 & -0.16 & 13.34 \\
\hline 0.327 & 5500 & 7.4041 & 2.89 & 0.65 & 0.42 & 1.76 & 0.43 & 0.33 & 0.10 & -0.21 & 13.80 \\
\hline 0.327 & 5000 & 7.4258 & 3.91 & 0.77 & 0.50 & 2.10 & 0.50 & 0.39 & 0.12 & -0.31 & 14.37 \\
\hline 0.327 & 4500 & 7.4451 & 5.95 & 0.91 & 0.59 & 2.30 & 0.59 & 0.35 & 0.07 & -0.44 & 15.00 \\
\hline 0.327 & 4000 & 7.4559 & 8.41 & 1.03 & 0.67 & 2.02 & 0.66 & 0.04 & -0.03 & -0.47 & 15.57 \\
\hline 0.327 & 3500 & 7.4622 & 11.39 & 1.13 & 0.72 & 1.27 & 0.68 & -0.21 & -0.20 & -0.32 & 16.02 \\
\hline 0.327 & 3000 & 7.4665 & 15.67 & 1.23 & 0.72 & 0.26 & 0.51 & -0.30 & -0.32 & -0.01 & 16.39 \\
\hline 0.327 & 2500 & 7.4701 & 23.95 & 1.33 & 0.61 & -0.96 & -0.10 & -0.20 & -0.51 & 0.39 & 16.79 \\
\hline 0.406 & 10000 & 7.5664 & 0.71 & 0.06 & 0.08 & 0.12 & 0.07 & 0.03 & -0.06 & -0.39 & 11.55 \\
\hline 0.406 & 9500 & 7.5722 & 0.82 & 0.10 & 0.11 & 0.24 & 0.10 & 0.05 & -0.05 & -0.35 & 11.75 \\
\hline 0.406 & 9000 & 7.5783 & 0.95 & 0.14 & 0.13 & 0.37 & 0.12 & 0.08 & -0.04 & -0.31 & 11.96 \\
\hline 0.406 & 8500 & 7.5838 & 1.11 & 0.19 & 0.16 & 0.51 & 0.15 & 0.11 & -0.03 & -0.27 & 12.18 \\
\hline 0.406 & 8000 & 7.5897 & 1.30 & 0.24 & 0.19 & 0.67 & 0.18 & 0.14 & -0.02 & -0.24 & 12.43 \\
\hline 0.406 & 7500 & 7.5952 & 1.55 & 0.29 & 0.22 & 0.84 & 0.22 & 0.18 & -0.01 & -0.21 & 12.69 \\
\hline 0.406 & 7000 & 7.6010 & 1.85 & 0.36 & 0.26 & 1.02 & 0.26 & 0.22 & 0.01 & -0.18 & 12.98 \\
\hline
\end{tabular}




\begin{tabular}{cccccccccccc}
\hline$M_{*} / \mathrm{M}_{\odot}$ & $\mathrm{T}_{\text {eff }}$ & Log $g$ & Edad (Gyr) & B-V & V-R & V-K & R-I & J-H & H-K & BC & $\mathrm{M}_{V}$ \\
\hline & & & & & & & & & & & \\
0.406 & 6500 & 7.6072 & 2.25 & 0.44 & 0.30 & 1.23 & 0.30 & 0.25 & 0.03 & -0.16 & 13.29 \\
0.406 & 6000 & 7.6139 & 2.77 & 0.53 & 0.36 & 1.47 & 0.36 & 0.29 & 0.06 & -0.16 & 13.66 \\
0.406 & 5500 & 7.6238 & 3.52 & 0.65 & 0.42 & 1.75 & 0.42 & 0.33 & 0.10 & -0.21 & 14.11 \\
0.406 & 5000 & 7.6374 & 5.00 & 0.77 & 0.50 & 2.09 & 0.50 & 0.38 & 0.12 & -0.31 & 14.66 \\
0.406 & 4500 & 7.6482 & 7.71 & 0.91 & 0.59 & 2.25 & 0.59 & 0.33 & 0.06 & -0.43 & 15.27 \\
0.406 & 4000 & 7.6543 & 10.83 & 1.02 & 0.66 & 1.92 & 0.66 & 0.01 & -0.06 & -0.44 & 15.80 \\
0.406 & 3500 & 7.6580 & 14.68 & 1.12 & 0.71 & 1.15 & 0.67 & -0.22 & -0.22 & -0.28 & 16.23 \\
0.406 & 3000 & 7.6607 & 20.37 & 1.21 & 0.71 & 0.16 & 0.47 & -0.30 & -0.33 & 0.02 & 16.60 \\
\hline
\end{tabular}

\subsection{Conclusiones}

Los cálculos presentados en este capítulo tienen como objetivo explorar la evolución de HeWDs. Los resultados están basados en un tratamiento muy detallado de modelos de atmósfera no-gris, lo cual permite derivar las condiciones externas de borde de manera precisa para los modelos evolutivos. El énfasis está puesto en las etapas avanzadas de la evolución de estos objetos, donde el modelado de su evolución es marcadamente dependiente del tratamiento que se haga de su atmósfera. Las fases evolutivas que llevan a la formación de HeWDs frías han sido exploradas en el capítulo 3, donde se encontró que la inclusión de la difusión de elementos en los cálculos evolutivos de las HeWDs lleva a que las envolturas de hidrógeno sean muy delgadas como para soportar una combustión nuclear estable que contribuya apreciablemente a la reserva energética de la estrella durante su evolución en la rama de enfriamiento. Aquí se mejoran dichos cálculos en base a un tratamiento detallado de la atmósfera incluido auto-consistentemente en el código evolutivo. Otra motivación ha sido contruir la función de luminosidad teórica para las HeWDs y proveer índices de color y magnitudes para estas HeWDs de manera consistente con la evolución estelar y la difusión de elementos.

En particular, hemos considerado modelos de HeWD de 0.406, 0.360, 0.327, 0.292, 0.242, 0.196 y $0.169 \mathrm{M}_{\odot}$, cuya evolución ha sido seguida desde el final de la fase de pérdida de masa durante la evolución pre-WD hasta luminosidades superficiales muy bajas.

Encontramos que cuando la $\mathrm{T}_{\text {eff }}$ desciende por debajo de $4000 \mathrm{~K}$, el espectro emergente de las HeWDs se vuelve más azul, como ha sido reportado previamente por otros investigadores. También analizamos el comportamiento de nuestros modelos de HeWDs en los diagramas colormagnitud y encontramos que las HeWDs con masas entre 0.18 y $0.30 \mathrm{M}_{\odot}$ pueden alcanzar el turn-off en sus colores y volverse azules nuevamente en edades menores a $15 \mathrm{Gyr}$, permaneciendo más brillantes que $M_{V} \approx 16.5$. Este es un resultado interesante ya que abre la posibilidad de que muchas HeWDs de baja masa puedan haber tenido tiempo suficiente para que hayan evolucionado más allá del punto de turn-off y presenten actualmente colores azules. Estas edades evolutivas relativamente cortas, son el resultado de la incorporación de la difusión en los modelos de evolución. La detección de WDs de baja masa azules a muy bajas $\mathrm{T}_{\text {eff }}$ serviría para poner sobre una base observacional firme nuestras predicciones teóricas. 


\section{Capítulo 5}

\section{Enanas blancas de baja masa. Progenitores de baja metalicidad}

Es bien sabido que la composición química de nuestra galaxia ha ido evolucionando a partir de una distribución de abundancias similar a la resultante del Big Bang, hasta la distribución actual, resultado de diversas etapas de procesamiento nuclear. Los cúmulos globulares galácticos, por ejemplo, son generalmente objetos viejos, caracterizados por metalicidades bajas. De esta manera, las estrellas que los componen deben tener, o haber tenido al comienzo de su evolución, una metalicidad bastante inferior a la solar, reflejo de la distribución de abundancias al momento de su formación. El estudio de la evolución de estas estrellas, entonces, debe ser realizado teniendo en cuenta la diferente composición química inicial correspondiente a estos objetos de baja metalicidad. La reciente detección de enanas blancas de baja masa en cúmulos globulares nos ha llevado a estudiar la evolución de estos objetos cuando sus progenitores son estrellas con metalicidades iniciales menores que la solar. Estos cálculos son el objeto del presente capítulo.

Los resultados presentados en este capítulo pueden encontrarse en Serenelli, Althaus, Rohrmann \& Benvenuto (2002).

\subsection{Introducción}

Durante los últimos años, las HeWDs han comenzado a ser detectadas en cúmulos abiertos y globulares (Anderson et al., 1997; Landsman et al., 1997; Edmonds et al., 1999). Más recientemente, Edmonds et al. (2001) han detectado ópticamente la HeWD compañera de un púlsar de milisegundo en el cúmulo globular (CG) 47 Tucanae. También, Taylor et al. (2001) han presentado evidencia observacional de la existencia de una secuencia de HeWDs en el CG NGC 6397. Una interpretación apropiada de las observaciones de HeWDs en los CGs requiere cálculos de secuencias evolutivas para metalicidades mucho más bajas que la metalicidad solar usualmente asumida en los modelos de estas estrellas. En este sentido, los presentes cálculos han sido diseñados para complementar el conjunto de cálculos evolutivos realizados para metalicidades solares (ver capítulos 3 y 4). En estos cálculos, además, se utiliza una versión actualizada del código de atmósferas, particularmente en lo que se refiere a la microfísica incluida. Esto nos permite presentar colores y magnitudes precisos para $\mathrm{T}_{\text {eff }}$ altas, donde los efectos de las opacidades por ensanchamiento de líneas no son despreciables. Además, los modelos iniciales de las HeWDs menos masivas han sido obtenidos mediante un tratamiento más detallado de las etapas de pérdida de masa que llevan a la formación de las HeWDs. 


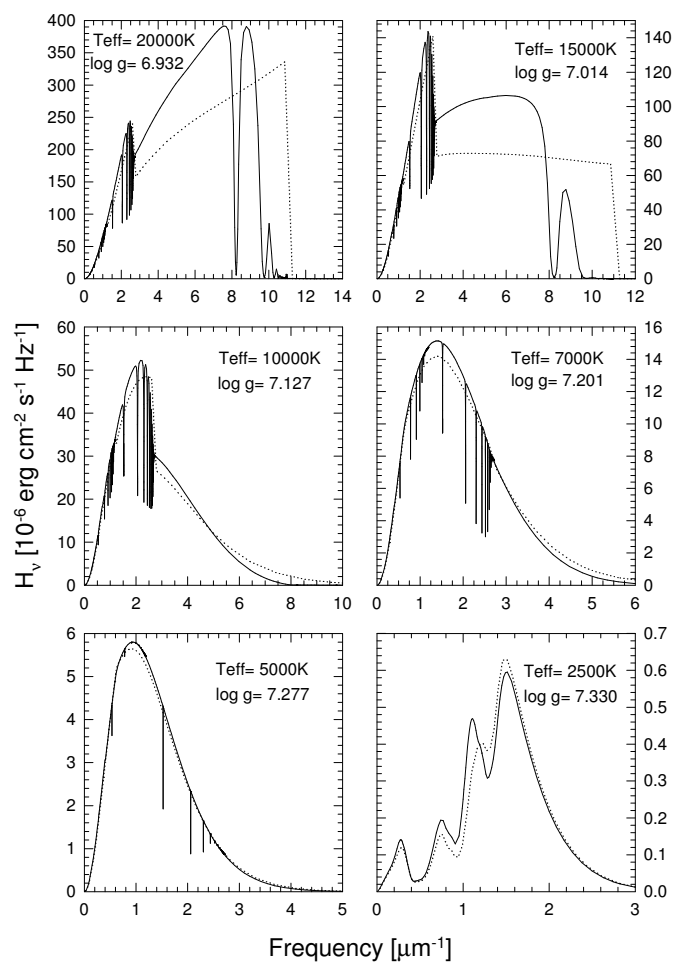

Figura 5.1: Espectro emergente para algunos modelos seleccionados de la secuencia de $0.280 \mathrm{M}_{\odot} \mathrm{y}$ $Z=0.0002$. Las $\mathrm{T}_{\text {eff }} \mathrm{y}$ las gravedades superficiales están indicadas en cada panel. Los modelos están caracterizados por envolturas de hidrógeno puro, resultantes de la difusión. Las líneas sólidas muestran los resultados del tratamiento mejorado incluyendo las opacidades de las líneas, modelo de gas no-ideal y opacidades CIA actualizadas. Las líneas de puntos indican los resultados obtenidos con la versión previa del modelo de atmósferas.

\subsection{Modelos de atmósfera}

Como se mencionó en el capítulo 4, el enfriamiento de las WDs es muy sensible al tratamiento de las condiciones de contorno externas. Por lo tanto, estan condiciones de contorno deben obtenerse a partir de un tratamiento detallado de la atmósfera de las WDs. Este es un aspecto importante en relación al enfriamiento de las HeWDs. Además, estos modelos atmosféricos permiten obtener una grilla de índices de color y magnitudes para nuestros modelos evolutivos. La estructura de los modelos atmosféricos está descripta en detalle en Rohrmann (2001). También, una descripción breve ha sido hecha en el capítulo 4. Un detalle de las mejoras introducidas en los presentes cálculos puede encontrarse en Rohrmann, Serenelli, Althaus \& Benvenuto (2002), aquí presentamos una descripción breve de las mismas. Con respecto a los cálculos del capítulo 4, las principales diferencias son las siguientes. Las opacidades CIA debidas a las colisiones $\mathrm{H}_{2}-\mathrm{H}_{2}, \mathrm{H}_{2}$-He, H-He están tomadas de Borysow, Jorgensen \& Fu (2001); Jorgensen et al. (2000) y Gustafsson \& Frommhold (2001), respectivamente. Las CIA representan una fuente de opacidad muy importante en el infrarrojo y dominan la forma del flujo emergente a bajas $\mathrm{T}_{\text {eff }}$. Las constantes de calibración usadas fueron derivadas en base al flujo sintético del modelo de Vega ( $\left.\mathrm{T}_{\text {eff }}=9400 \mathrm{~K}, \log g=3.95\right)$ calculado por Kurucz (1979).

La versión del código de atmósferas utilizada en estos cálculos presenta mejoras importantes, que nos han permitido obtener colores y magnitudes precisas para etapas evolutivas tempranas 
y avanzadas en el enfriamiento de las HeWDs. Específicamente, las opacidades de las líneas de las series de Lyman, Balmer y Paschen, y las absorciones del pseudo-continuo han sido incluidas en los cálculos. También, los efectos no-ideales en el cálculo del equilibrio químico de gases mezcla de higrógeno y helio son tenidos en cuenta a través del formalismo ocupacional de Hummer \& Mihalas (1988) junto con una versión modificada de la simulación óptica de Däppen, Anderson \& Mihalas (1987) para determinar las opacidades en gases no-ideales. Para más detalles referirse a Rohrmann, Serenelli, Althaus \& Benvenuto (2002).

En la figura 5.1 se muestra el flujo emergente de nuestro modelo de HeWD de $0.280 \mathrm{M}_{\odot}$ para algunos puntos seleccionados de su evolución. Las predicciones de los cálculos presentes se muestran en líneas sólidas y son comparadas con el tratamiento dado anteriormente (ver capítulo 4). Notar que a $\mathrm{T}_{\text {eff }}$ mayores que $7000 \mathrm{~K}$, las líneas de hidrógeno modifican fuertemente la forma de la distribución del flujo, de modo que es esperable que sus efectos sean notorios en los colores U y B. A bajas $\mathrm{T}_{\text {eff }}$, el espectro emergente está dominado por las opacidades CIA, y la mayor parte de la radiación es forzada a emerger a frecuencias mayores en comparación a las predicciones del cuerpo negro. El resultado, ya mencionado en el capítulo 4, es que los colores infrarrojos presentan un turn-off pronunciado hacia el azul cuando la $\mathrm{T}_{\text {eff }}$ cae por debajo de $\approx 4000 \mathrm{~K}$. Finalmente, notar que la distribución del flujo difiere un poco con respecto al tratamiento previo. Esto se debe en parte a la inclusión de los efectos no-ideales y al uso de las opacidades CIA actualizadas.

\subsection{Modelos iniciales}

La física constitutiva incorporada en el CEELP para los presentes cálculos es la misma que la utilizada en los cálculos presentados en los capítulos anteriores. Como se mencionó también en el capítulo 3, la estructura termomecánica de los modelos de HeWD no depende fuertemente de la manera en que la envoltura de hidrógeno es removida, y las perturbaciones introducidas se relajan antes de que el modelo ingrese en la rama de enfriamiento. Sin embargo, en el caso de los modelos menos masivos, donde las escalas temporales de la fase de pérdida de masa y de evolución del progenitor son comparables, el tratamiento de la pérdida de masa puede influir en la estructura de los modelos iniciales de HeWDs. Para considerar estas etapas de manera apropiada, el CEELP ha sido modificado para simular la evolución del progenitor de HeWD durante las fases de pérdida de masa de un modo adecuado.

Las HeWDs, y particularmente las de masa baja ${ }^{1}$, son el resultado de la evolución de estrellas de masa baja en sistemas binarios cercanos. La pérdida de masa en estos sistemas ocurre cuando el progenitor de HeWD llena su lóbulo de Roche como resultado de su evolución. El CEELP ha sido modificado para que durante las etapas de pérdida de masa, la tasa de pérdida de masa se ajuste de manera que el radio de la estrella sea igual al del lóbulo de Roche $r_{L}$. Esto nos permite simular la evolución de la estrella y de la tasa de pérdida de masa de manera precisa durante la fase en que el progenitor de HeWD pierde su envoltura de hidrógeno. Es bien sabido, además, que el semieje de los sistemas binarios cambia con el tiempo debido a diversos efectos.

En vista de lo mencionado más arriba, es importante conocer la evolución temporal del lóbulo de Roche durante la evolución del progenitor. Para esto hemos considerado diversos mecanismos que producen cambios en el tamaño del lóbulo de Roche. Siguiendo a Eggleton

\footnotetext{
${ }^{1}$ Castellani \& Castellani (1993) encuentran que HeWDs masivas $\left(\approx 0.4 \mathrm{M}_{\odot}\right)$ pueden ser el resultado de la evolución de estrellas aisladas, donde la HeWD es el resultado de la pérdida de la envoltura de hidrógeno en progenitores de metalicidad baja por efectos de los vientos estelares
} 
(1983), el radio del lóbulo de Roche está dado mediante la aproximación analítica

$$
r_{L}=a \frac{0.49 q^{2 / 3}}{0.6 q^{2 / 3}+\ln \left(1+q^{1 / 3}\right)},
$$

donde $a$ es el semieje de la órbita y $q$ es el cociente de masas de las componentes. Seguir la evolución temporal de $r_{L}$ implica entonces seguir la evolución temporal del semieje $a$ de la órbita. Para este fin, hemos asumido que la pérdida de masa del progenitor es completamente no-conservativa ${ }^{2}$, es decir que toda la masa perdida por el progenitor de HeWD es perdida del sistema y además se lleva también todo su momento angular intrínseco. La formulación adoptada es la de Podsiadlowski, Joss \& Hsu (1992)

$$
\frac{\dot{J}_{M L}}{J}=\frac{M}{M_{1} M_{2}} \dot{M}_{1} \mathrm{yr}^{-1} .
$$

Aquí, $J$ es el momento angular total, $\dot{J}_{M L}$ su derivada temporal causada por la pérdida de masa y $M$ la masa total del sistema. Los subíndices 1 y 2 se refieren al progenitor de HeWD y a la compañera respectivamente. Finalmente, $\dot{M}_{1}$ es la tasa de pérdida de masa del progenitor. También hemos incluido las pérdidas de momento angular por radiación de ondas gravitatorias (Landau \& Lifshitz, 1971)

$$
\frac{\dot{J}_{G R}}{J}=-8.5 \times 10^{-10} \frac{M_{1} M_{2} M}{a^{4}} \mathrm{yr}^{-1}
$$

que es una contribución importante a la pérdida de momento angular en binarias de períodos muy cortos. Finalmente, el frenado magnético que ocurre cuando se desarrolla una envoltura convectiva también ha sido incluido de acuerdo a Sarna, Ergma \& Antipova (2000)

$$
\frac{\dot{J}_{M B}}{J}=-3 \times 10^{-7} \frac{M^{2} R_{1}^{2}}{M_{1} M_{2} a^{5}} \mathrm{yr}^{-1} .
$$

Para seguir la evolución temporal de $J$, integramos la ecuación

$$
\frac{\dot{J}}{J}=\frac{\dot{J}_{M L}}{J}+\frac{\dot{J}_{G R}}{J}+\frac{\dot{J}_{M B}}{J}
$$

y con la ayuda de la tercera ley de Kepler obtenemos finalmente a y por consiguiente $r_{L}$. El presente tratamiento para la pérdida de masa no es autoconsistente ya que la tasa de pérdida de masa no es incorporada como una nueva cantidad desconocida que debe ser calculada durante el procedimiento iterativo en el esquema de Henyey usado para resolver las ecuaciones de estructura estelar, sino que es fijada de antemano durante el calculo de un modelo evolutivo y una vez que éste convergió es recalculada para el modelo siguiente. Sin embargo, queremos recalcar que está más allá de nuestros objetivos analizar las propiedades (relaciones entre períodos inicial y final, masas, semiejes) de los sistemas binarios que llevan a la formación de sistemas binarios HeWD-púlsar de milisegundo, sino simplemente obtener modelos iniciales confiables para estudiar las propiedades de enfriamiento de las HeWDs como así también estudiar las propiedades fotométricas de estas estrellas.

Para estudiar los efectos de la metalicidad en la evolución de las HeWDs, hemos realizado dos conjuntos de cálculos evolutivos. En uno de ellos hemos considerado HeWDs provenientes

\footnotetext{
${ }^{2}$ Los comapñeros de HeWDs son generalmente objetos compactos y cálculos detallados indican que en estos casos prácticamente toda la masa es perdida del sistema.
} 


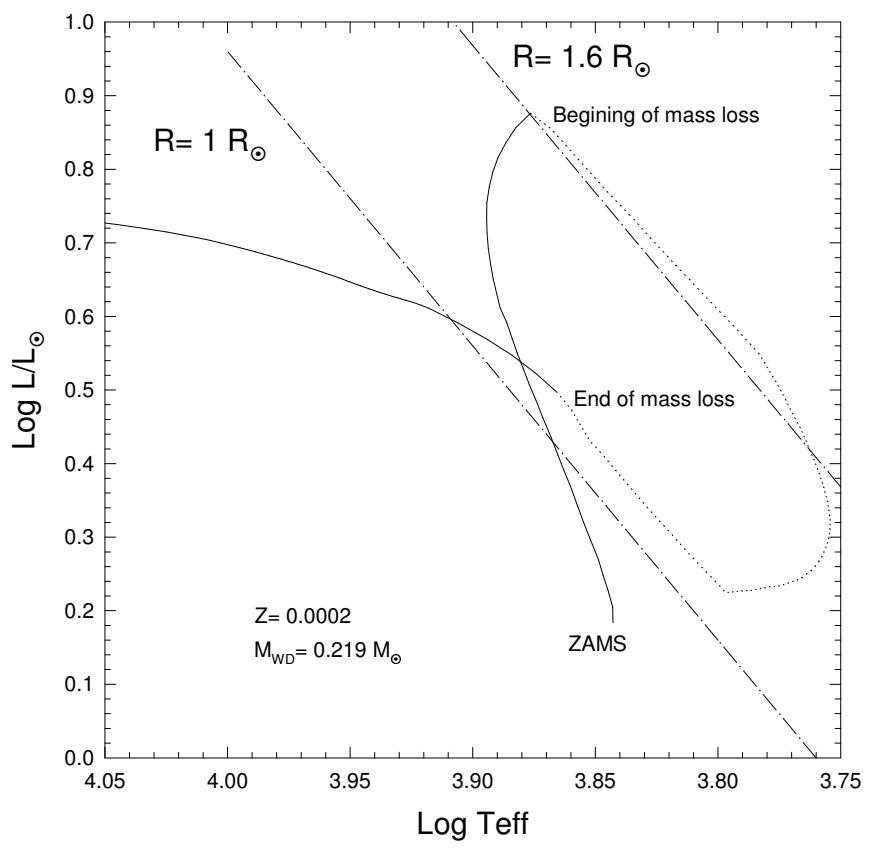

Figura 5.2: Diagrama Hertzsprung-Russell para el progenitor del modelo de HeWD de 0.219 $\mathrm{M}_{\odot} \mathrm{y}$ $Z=0.0002$. La línea de puntos indica la evolución durante la fase de pérdida de masa, que comienza cuando la estrella llena su lóbulo de Roche por primera vez a $R=1.6 R_{\odot}$. Los semiejes inicial y final son 5.25 y $4.6 \mathrm{R}_{\odot}$. Los períodos inicial y final son $0.9 \mathrm{~d}$ en ambos casos.

de progenitores con $Z=0.001$ y en el segundo de progenitores de $Z=0.0002$ apropiados para medios muy pobres en metales. Estos valores de metalicidad han sido elegidos de modo tal que resulten representativos de las bajas metalicidades comunes entre los CGs. En el caso de $Z=0.001$ los modelos de HeWD resultantes tienen 0.172, 0.183, 0.197, 0.230, 0.244, 0.300, $0.336,0.380,0.390,0.422,0.449 \mathrm{M}_{\odot}, \mathrm{y}$ en el caso de $Z=0.0002$ sus masas son $0.199,0.209$, $0.219,0.225,0.243,0.266,0.280,0.300,0.319 \mathrm{M}_{\odot}$.

Los modelos iniciales se han calculado de dos maneras diferentes. Si la pérdida de masa comienza cuando el progenitor se encuentra en la rama de las gigantes rojas, entonces se sigue el mismo procedimiento empleado en los modelos usados en el capítulo 3, es decir, la tasa de pérdida de masa es fijada ad-hoc. Cuando la pérdida de masa comienza antes de que la estrella alcance la rama de las gigantes, es decir, inmediatamente después de dejar la secuencia principal, empleamos el método descripto aquí. De este modo, los modelos iniciales para las secuencias menos masivas que $0.2 \mathrm{M}_{\odot}$ para $Z=0.001$, y menos que $0.23 \mathrm{M}_{\odot}$ en el caso de $Z=0.0002$ fueron obtenidos según el procedimiento descripto aquí. En todos los casos se supuso que el progenitor es una estrella de $1 \mathrm{M}_{\odot}$ compañera de un objeto de $1.4 \mathrm{M}_{\odot}$, masa representativa de la de un púlsar. Para ilustrar los resultados, mostramos el track evolutivo del progenitor de $0.219 \mathrm{M}_{\odot}$ y $Z=0.0002$ en la figura 5.2. Notar que al comienzo de la fase de pérdida de masa la evolución ocurre a radio casi constante. La desviación hacia radios ligeramente mayores se debe a que el semieje aumenta ligeramente como resultado de la pérdida de masa por parte de la componente menos masiva del sistema. A medida que la evolución prosigue, la estrella desarrolla una envoltura convectiva por lo que el frenado magnético comienza a jugar un rol preponderante y la órbita (y por lo tanto el lóbulo de Roche) comienza a estrecharse. Durante 
la fase final de pérdida de masa, la evolución nuevamente ocurre a radio casi constante hasta que finalmente la envoltura se contrae dentro del lóbulo de Roche finalizando así la pérdida de masa. En la tabla 5.3 se listan las proncipales características para algunos de los progenitores de HeWD menos masivos. Para un modelo de HeWD dado, listamos la metalicidad, la abundancia superficial de hidrógeno al final de la pérdida de masa y los períodos y semiejes inicial y final. Los resultados están en buen acuerdo con los obtenidos mediante un tratamiento auto-consistente de la pérdida de masa (Sarna, Ergma \& Antipova, 2000).

Table 5.1: Principales características de algunos modelos pre-WD de baja masa.

\begin{tabular}{clccccc}
\hline $\mathrm{M}_{\mathrm{f}}\left[\mathrm{M}_{\odot}\right]$ & \multicolumn{1}{c}{$\mathrm{Z}$} & $\mathrm{X}_{\mathrm{s}}$ & $\mathrm{P}_{\mathrm{i}}[\mathrm{d}]$ & $a_{\mathrm{i}}\left[\mathrm{R}_{\odot}\right]$ & $\mathrm{P}_{\mathrm{f}}[\mathrm{d}]$ & $a_{\mathrm{f}}\left[\mathrm{R}_{\odot}\right]$ \\
\hline 0.199 & 0.0002 & 0.390 & 0.70 & 4.44 & 0.36 & 2.47 \\
0.219 & 0.0002 & 0.435 & 0.90 & 5.25 & 0.90 & 4.60 \\
0.172 & 0.001 & 0.357 & 0.95 & 5.44 & 0.26 & 1.96 \\
0.183 & 0.001 & 0.391 & 1.02 & 5.71 & 0.43 & 2.79 \\
0.197 & 0.001 & 0.426 & 1.05 & 5.82 & 0.85 & 4.40 \\
\hline
\end{tabular}

\subsection{Resultados}

Cualitativamente, la evolución de las HeWDs con progenitores de baja metalicidad no es marcadamente diferente del caso de HeWDs con progenitores de metalicidad solar (resultados del capítulo 3). En efecto, en el caso de HeWDs de baja metalicidad encontramos que los objetos con masas por encima de cierto valor límite experimentan varios episodios de flashes termonucleares que afectan su historia de enfriamiento posterior. Sin embargo, el contenido de metales de las HeWD juega un papel importante en su evolución, en particular con respecto la ocurrencia y las características de los flashes de hidrógeno vía el ciclo CNO (nos referiremos a ellos simplemente como flashes CNO, para diferenciarlos de los flashes que ocurren vía la cadena protón-protón, que denominaremos flashes PP). En términos generales, los flashes de CNO son menos intensos cuanto menor es la metalicidad de la estrella. Esta es la razón por la cual los loops en el HRD inducidos por los flashes de CNO son marcadamente menos extendidos en el caso de los modelos de metalicidad más baja. En las figuras 5.3 y 5.4 se muestran los tracks evolutivos en el HRD para algunas de las secuencias de $Z=0.0002$ y $Z=0.001$ respectivamente. Es importante mencionar que la masa límite para la ocurrencia de los flashes de CNO depende de la metalicidad asumida para los modelos. Específicamente, para $Z=0.001$ y $Z=0.0002$ encontramos que esta masa límite $\left(M_{\mathrm{th}}\right)$ es $M_{\mathrm{th}} \approx 0.22$ y $0.26 \mathrm{M}_{\odot}$ respectivamente (tener en cuenta que en el caso de metalicidad solar $M_{\text {th }} \approx 0.18 \mathrm{M}_{\odot}$; ver capítulo 3 ). Los modelos menos masivos que $M_{\mathrm{th}}$, entonces, no sufren flashes nucleares de CNO. Con respecto a la masa máxima para la cual ocurren estos flashes, nuestro modelo más masivo con $Z=0.0002$ tiene $0.319 \mathrm{M}_{\odot}$ y es inestable, mientras que en el caso de $Z=0.001$, encontramos flashes de CNO en el modelo de $0.422 \mathrm{M}_{\odot}$ pero no en el de $0.449 \mathrm{M}_{\odot}$. El rango de masas en el cual los flashes nucleares tienen lugar es dependiente del hecho que la difusión se considerada o no en los cálculos, como se encontró previamente en los modelos de metalicidad solar (capítulo 3). Por ejemplo, en el caso de $Z=0.0002$ sólo los modelos de $0.280,0.300$ y $0.319 \mathrm{M}_{\odot}$ sufren flashes de CNO cuando la difusión no es tenida en cuenta en los cálculos. Para el otro valor de metalicidad considerado, $Z=0.001$, los modelos en el rango entre 0.24 y $0.35 \mathrm{M}_{\odot}$ aproximadamente sufren estas 

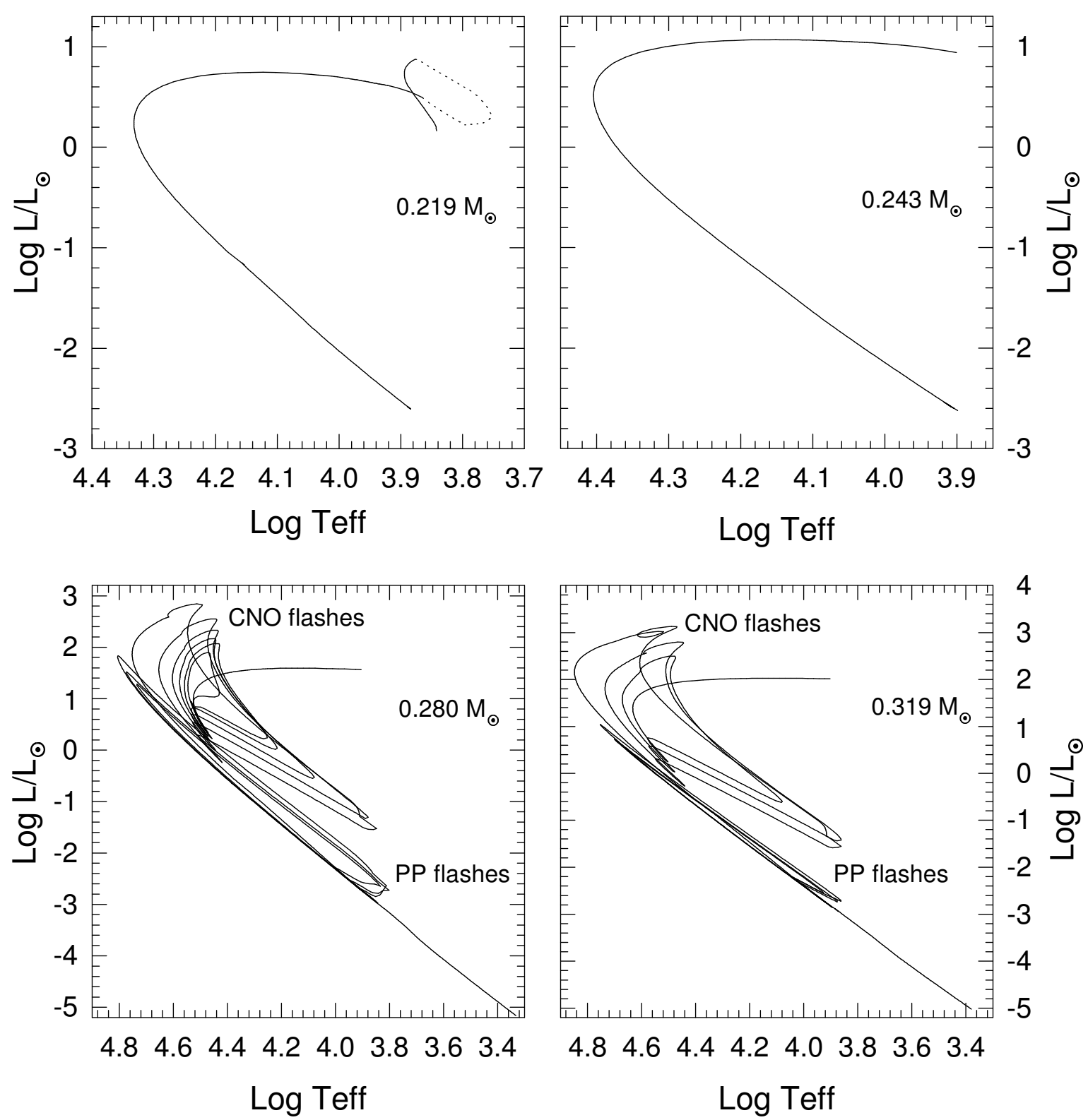

Figura 5.3: Tracks evolutivos en el HRD para algunas masas seleccionadas de nuestra grilla de modelos de HeWDs para $Z=0.0002$. Las masas están indicadas en los gráficos. Para el modelo de $0.219 \mathrm{M}_{\odot}$, se muestra también la evolución pre-WD (como en la figura 5.2). En los modelos de 0.219 y $0.243 \mathrm{M}_{\odot}$ no ocurren flashes termonucleares. Sin embargo, los modelos de 0.280 y $0.319 \mathrm{M}_{\odot}$ sufren algunos flashes de hidrógeno dominados por el ciclo CNO, apenas finalizada la evolución pre-WD. Estos flashes se manifiestan en el HRD como moderadas excursiones hacia el rojo. Después de estos flashes los modelos se enfrían pero cuando la $\mathrm{T}_{\text {eff }}$ alcanza aproximadamente $8000 \mathrm{~K}$ otra secuencia de flashes tiene lugar, aunque en este caso completamente dominada por la combustión del hidrógeno vía la cadena protónprotón. En el HRD, estos modelos evolucionan hacia arriba y hacia abajo con radio casi constante. 

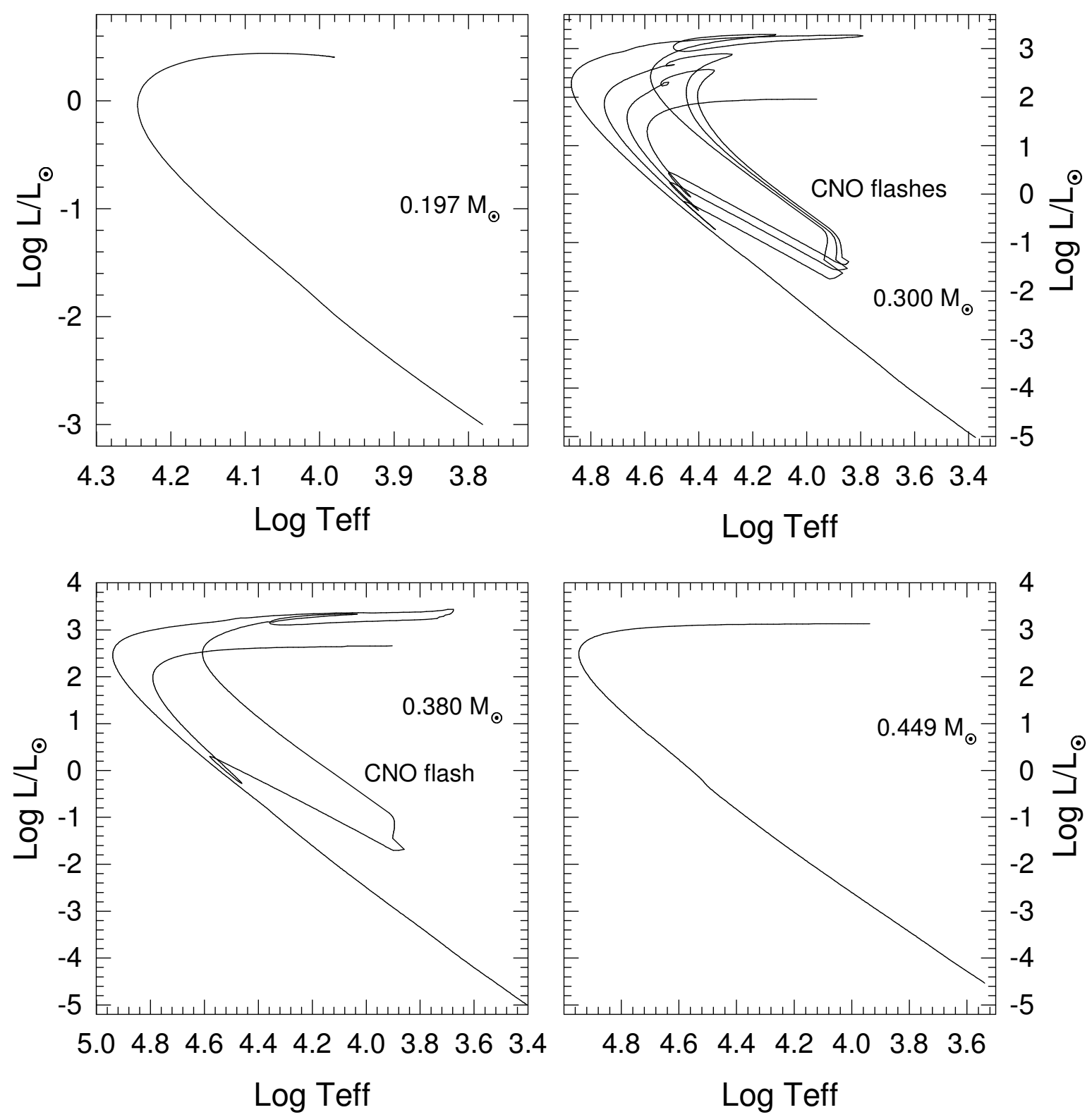

Figura 5.4: Tracks evolutivos en el HRD para algunas masas seleccionadas de nuestra grilla de modelos de HeWDs para $Z=0.001$. El modelo menos masivos que se muestra no sufre ningún flash termonuclear y se enfría muy lentamente por efectos de la combustión nuclear residual, que es la principal fuente de energía de la estrella. Para valores intermedios de la masa estelar, representados aquí por el modelo de $0.244 \mathrm{M}_{\odot}$, la difusión de elementos induce la ocurrencia de varios flashes nucleares dominados por la combustión vía el ciclo CNO. Para masas mayores el número de flashes CNO disminuye $\left(0.380 \mathrm{M}_{\odot}\right)$ y finalmente para el mayor valor de la masa no encontramos ningún flash termonuclear $\left(0.449 \mathrm{M}_{\odot}\right)$. 
inestabilidades termonucleares.

Una característica que encontramos en nuestros cálculos es la existencia de inestabilidades térmicas en los modelos con $Z=0.0002$ en etapas más avanzadas de la evolución que aquellas en las que ocurren los flashes de CNO. Estas inestabilidades están relacionadas con la combustión del hidrógeno de manera inestable vía las cadenas protón-protón (PP) que dominan la combustión del hidrógeno a edades avanzadas. La existencia de estos flashes PP puede entenderse en función de la envoltura más gruesa de hidrógeno que caracteriza a los modelos de tan baja metalicidad. Esto es el resultado de que la combustión previa vía el ciclo CNO es deficiente debido al bajo contenido de metales de la envoltura de la estrella durante la evolución pre-WD así como durante los flashes CNO. Después de la ocurrencia de los flashes CNO, la extensión radial del límite externo de la región donde ocurre la combustión del hidrógeno va disminuyendo a medida que la evolución de la estrella prosigue. Al mismo tiempo, algo del hidrógeno se difunde hacia el interior estelar por efectos de la difusión química, alcanzando regiones más calientes. Cuando el shell que se está quemando se vuelve lo suficientemente delgado, la combustión nuclear se torna inestable, de acuerdo a lo predicho por el criterio de inestabilidad de Kippenhahn \& Weigert (1990) (ver Driebe et al., 1999 para una aplicación de este criterio en el contexto de los flashes de CNO en HeWDs)

$$
C^{*}=C_{P}\left(1-\frac{4 \nabla_{a d} \delta}{4 \alpha-\frac{R_{\text {shell }}}{D}}\right)>0
$$

donde $C_{*}$ y $C_{P}$ son los calores específicos gravotérmicos y a presión constante respectivamente, $\nabla_{a d}$ es el gradiente adiabático, $\alpha=\left(\frac{\partial \ln \rho}{\partial \ln P}\right)_{T}, \delta=-\left(\frac{\partial \ln \rho}{\partial \ln T}\right)_{P}, R_{\text {shell }}$ es la coordenada radial del shell y $D$ su extensión radial (definida como el punto donde la liberación de energía nuclear cae por debajo de 0.001 veces de su valor máximo). En nuestros cálculos encontramos que este criterio es satisfecho por nuestros modelos al comienzo de los flashes de PP. Esto se debe a los valores grandes alcanzados por la cantidad $R_{\text {shell }} / D$, que excede 10 al comienzo de los flashes, mientras que $\alpha$ y $\delta$ valen cerca de 0.85 y 0.6 respectivamente.

Los flashes PP difieren de los de CNO en algunos aspectos. Por ejemplo, en la mayor parte de los casos los flashes de PP son menos intensos que los de CNO y durante los episodios de flash la mezcla convectiva de las capas externas de la estrella ocurre en menos casos con respecto a los flashes de CNO. Una característica interesante de estos flashes es que una vez que el flash se inicia, la estrella es forzada a moverse en el diagrama HR siguiendo líneas de radio casi constante, cercanas al track de enfriamiento. También, durante estos flashes la cantidad de hidrógeno total de la estrella es apenas reducida con el resultado que la estrella experimenta en general varios flashes nucleares de este tipo. En las secuencias con $Z=0.001$ sólo aquellos modelos que no sufren flashes CNO experimentan flashes PP, pero estos ocurren a edades extremadamente altas. Todas nuestras secuencias de $Z=0.0002$ están caracterizadas por flashes PP aún cuando hayan experimentado flashes CNO previamente. El punto importante para enfatizar es que los flashes PP son inducidos por la difusión química que opera difundiendo hacia el interior de la estrella la cola de la distribución de hidrógeno en la base de la envoltura, a regiones suficientemente calientes como para que el hidrógeno se encienda. Excepto para los modelos menos masivos $\left(M \leq 0.25 \mathrm{M}_{\odot}\right)$, los flashes PP en las secuencias con $Z=0.0002$ tienen lugar entre 2 y 4 Gyr después del fin de la etapa de pérdida de masa pre-WD. En general, cuanto más masivo es el modelo, antes en su vida se producen los flashes de PP. Es importante 

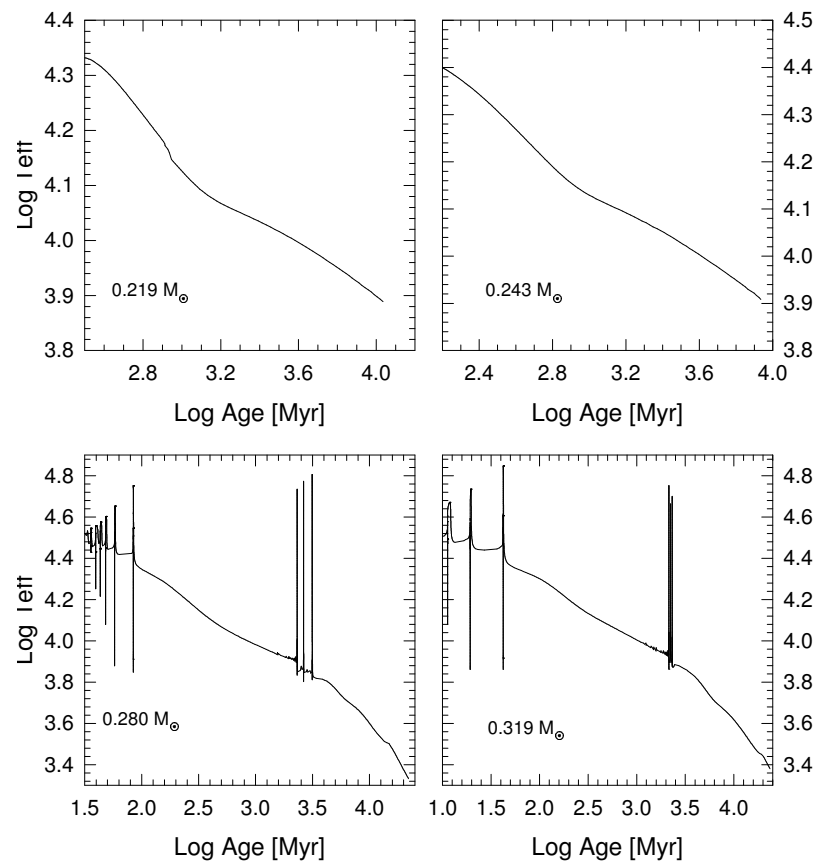

Figura 5.5: $\mathrm{T}_{\text {eff }}$ como función de la edad para las mismas secuencias que en la figura 5.3. En los modelos más masivos, los picos corresponden a los estados evolutivos durante los episodios de flash. Es claro que los modelos que experimentan estos flashes alcanzan $\mathrm{T}_{\text {eff }}$ muy bajas en menos de 15 Gyr.
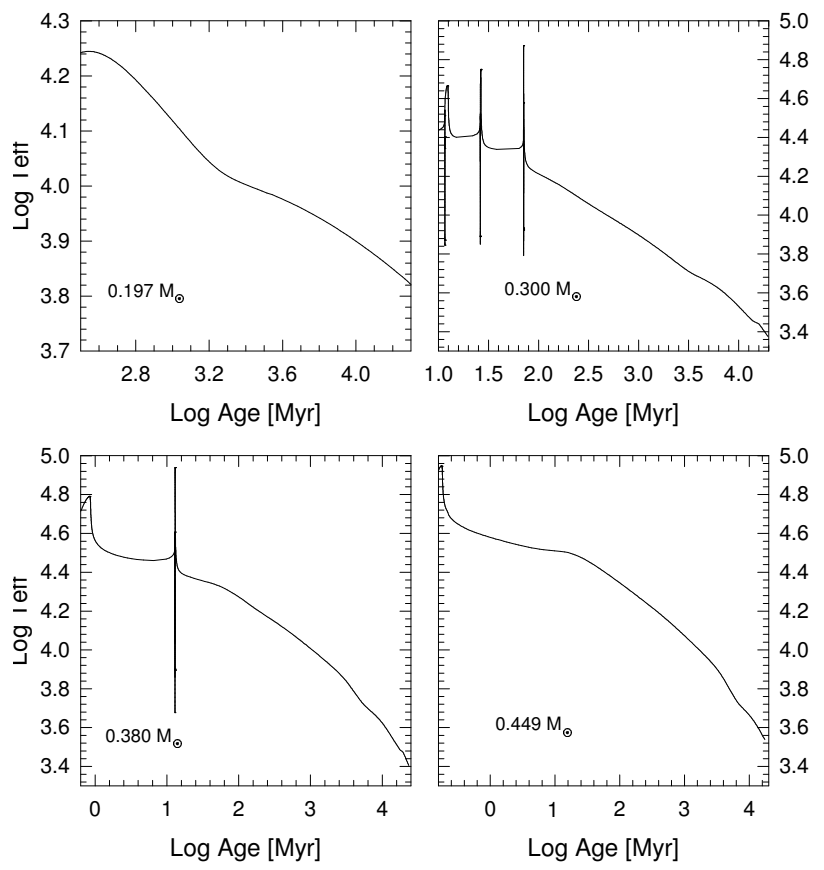

Figura 5.6: $\mathrm{T}_{\text {eff }}$ como función de la edad para las mismas secuencias que en la figura 5.4. Los picos corresponden a los estados evolutivos durante los episodios de flash. Igual que en la figura 5.6, los modelos caracterizados por la ocurrencia de flashes alcanzan $\mathrm{T}_{\text {eff }}$ muy bajas en menos de 15 Gyr. La secuencia de $0.449 \mathrm{M}_{\odot}$ tiene escalas de tiempo de enfriamiento cortas aún en ausencia de flashes nucleares. 


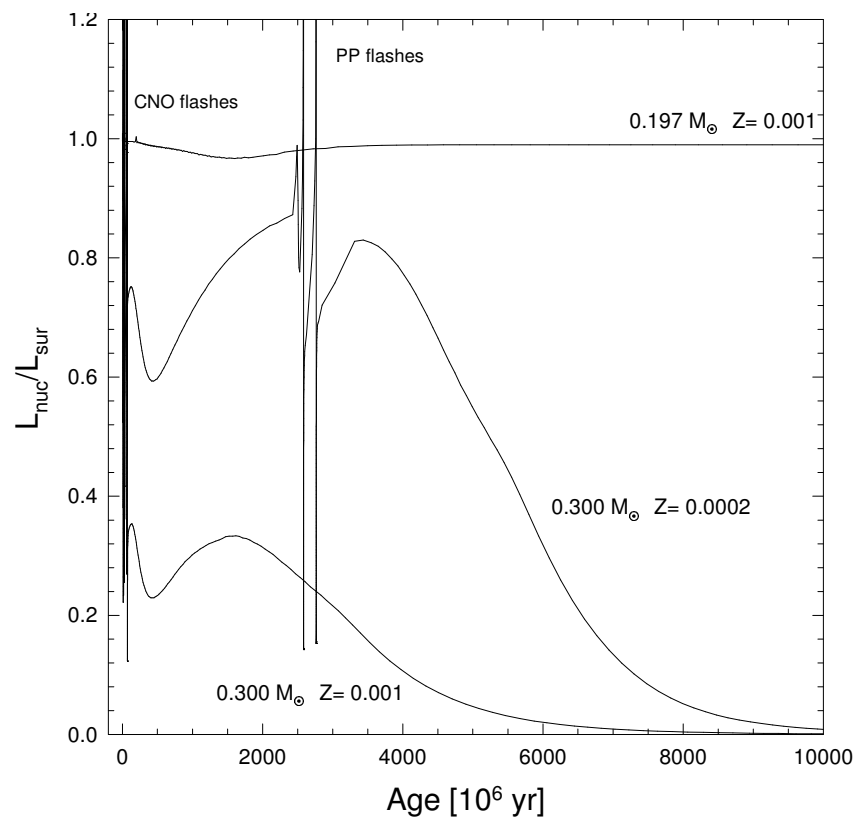

Figura 5.7: Luminosidad por combustión del hidrógeno en términos de la luminosidad superficial como función de la edad para nuestros modelos de $0.197(Z=0.001)$ y $0.300 \mathrm{M}_{\odot}(Z=0.001$ y $Z=0.0002)$. Notar que para la masa más baja, la ausencia de flashes termonucleares hace que la luminosidad superficial se deba casi exclusivamente a la producida por la combustión estable del hidrógeno. En contraste, para modelos más masivos, la combustión estable que sigue a los episodios de flash contribuye sólo parcialmente a la reserva energética de la estrella. Notar también que cuando la metalicidad es mayor, la contribución de la combustión residual del hidrógeno resulta menos importante.

remarcar que la ocurrencia de estos flashes se restringe a los modelos de baja metalicidad ya que ningún modelo de metalicidad solar (capítulo 3) los experimenta, y en el caso de $Z=0.001$ sólo los modelos de 0.172 y $0.183 \mathrm{M}_{\odot}$ lo hacen.

En las figuras 5.5 y 5.6 se muestra la $\mathrm{T}_{\text {eff }}$ como función de la edad de enfriamiento para las mismas secuencias que en las figuras 5.3 y 5.4 respectivamente. Notar que la ausencia de flashes $\mathrm{CNO}$ en los modelos menos masivos conduce a una velocidad evolutiva lenta durante el enfriamiento de la estrella y por lo tanto a edades grandes. Esto resulta así porque la combustión del hidrógeno vía PP es una fuente muy importante de energía para estos modelos durante casi toda su evolución. Por el contrario, la existencia de flashes CNO reduce notablemente la envoltura de hidrógeno de los modelos de manera que la combustión estable del hidrógeno durante el enfriamiento de los modelos resulta despreciable como fuente de enrgía. De acuerdo con esto, estos modelos están caracterizados por escalas evolutivas cortas. Esta dicotomía en las edades de enfriamiento entre los modelos que sufren flashes CNO y los modelos que no los sufren tiene las mismas características que los encontrados para modelos de HeWD de metalicidad solar (ver capítulo 3). Sin embargo, hay que notar que esta dicotomía es menos marcada en los modelos con muy bajo contenido de metales. Para poner esta afirmación en números, mencionemos que para alcanzar un valor de $\mathrm{T}_{\text {eff }}=8000 \mathrm{~K}$, los modelos con $0.219,0.243,0.280$ y $0.319 \mathrm{M}_{\odot}(Z=0.0002)$ necesitan alrededor de 10, 8.7, 3.1 y 2.3 Gyr respectivamente. En el caso de $Z=0.001$, los modelos con $0.197,0.300,0.319$ y $0.449 \mathrm{M}_{\odot}$ necesitan unos $10,1,2.2$ y 3.1 Gyr respectivamente. La dependencia de la historia del enfriamiento con la metalicidad de 
los modelos puede entenderse mediante una inspección de la figura 5.7 en la cual se muestra la dependencia temporal de la luminosidad por combustión del hidrógeno de las secuencias de $0.300 \mathrm{M}_{\odot}$ y $Z=0.001$ y $Z=0.0002$. En el caso de $Z=0.001$, la combustión estable vía PP alcanza una contribución máxima del 35\% después de 1.5 Gyr de haber ocurrido los flashes CNO. Después de alcanzar esta máximo, la combustión nuclear se vuelve menos importante a medida que la evolución continúa. Por ejemplo, a los $4 \mathrm{Gyr}$, sólo contribuye con menos del $10 \%$ a la luminosidad estelar. En contraste, en el caso de la secuencia con $Z=0.0002$, la combustión nuclear estable contribuye apreciablemente aún para edades de alrededor de 6 Gyr. Después de los flashes de CNO, esta contribución a la luminosidad superficial de la estrella es mayor al $60 \%$ durante los primeros 5 Gyr. Esta contribución cae bastante abruptamente en las etapas sucesivas de la evolución, alcanzando un $10 \%$ a los 7 Gyr. Con respecto a esta figura, mencionemos también que se ha incluido la secuencia de $0.197 \mathrm{M}_{\odot}$ y $Z=0.001$ que no sufre episodios de flash. En este caso la luminosidad de la estrella está completamente soportada por la combustión estable del hidrógeno durante toda la rama de enfriamiento como puede verse en la figura 5.7.

En las figuras 5.8 y 5.9 se muestran las isocronas para algunas edades seleccionadas en el plano masa- $T_{\text {eff }}$ para nuestras secuencias evolutivas de HeWDs de $Z=0.001$ y $Z=0.0002$ respectivamente. Notar que en los valores $M_{\text {th }}$ respectivos, los gráficos de isocronas muestran una discontinuidad pronunciada. Esto no resulta sorprendente en vista de la dicotomía en las edades discutida previamente. El gráfico correspondiente a las secuencias $Z=0.001$ es muy similar al de los modelos con un contenido de metales solar (Althaus, Serenelli \& Benvenuto, 2001b). Esto es particularmente cierto con respecto a la existencia de una dicotomía en las edades en $M_{\text {th }} \approx 0.22 \mathrm{M}_{\odot}$ para este caso. Creemos que futuras observaciones de WDs de baja masa con púlsares de milisegundo como compañeros formadas en medios de baja metalicidad podrán, eventualmente, poner una base observacional para nuestras predicciones teóricas ${ }^{3}$. Otra característica notoria de la figura 5.8 es la existencia de un máximo local en las isocronas en las secuencias de 0.380 y $0.390 \mathrm{M}_{\odot}$. Este comportamiento es el resultado del rol jugado por la combustión estable del hidrógeno vía PP después de los flashes CNO. Específicamente, los modelos de 0.380 y $0.390 \mathrm{M}_{\odot}$ experimentan sólo un flash de CNO pues la envoltura remanente es demasiado delgada como para que otro flash CNO ocurra. Sin embargo, las envolturas son lo suficientemente gruesas como para mantener una liberación de energía nuclear apreciable, enlenteciendo de esta manera la evolución de estos objetos durante las etapas intermedias de su evolución. Finalmente, notar el aumento de la $\mathrm{T}_{\text {eff }}$ para una edad fija para la secuencia más masiva, que no sufre ningún flash termonuclear, lo que resulta en edades comparativamente grandes. La situación en el caso de las secuencias con $Z=0.0002$ es cualitativamente similar, aunque la dicotomía en las edades es menos marcada que en el caso previo y está localizada entre $0.25-0.26 \mathrm{M}_{\odot}$. Como se explicó previamente, esto es el resultado de que en este caso, durante los flashes CNO, las reacciones de este ciclo son menos eficientes que para metalicidades más altas por lo que la envoltura de hidrógeno no es drásticamente consumida.

La evolución de nuestros modelos en el diagrama color-magnitud (CMD) se ilustra en la figura 5.10, que muestra la magnitud visual absoluta $M_{V}$ para nuestros modelos como función de los índices de color $U-V$ y $V-I$. Notar que los modelos que sufren flashes CNO muestran un turn-off en el índice de color $V-I$ a $M_{V} \approx 16$ y $V-I \approx 1.4$. Este turn-off, que resulta de la fuerte influencia de las opacidades CIA del hidrógeno molecular, es alcanzado en menos de $15 \mathrm{Gyr}$, principalemte por los modelos con $Z=0.001$. Después de alcanzar el turn-off,

\footnotetext{
${ }^{3}$ Un soporte tentativo para la existencia de la dicotomía en las edades está dado por las HeWDs de campo compañeras de PSR B1855+09, PSR J0437-4715 y PSR J1012+5307.
} 


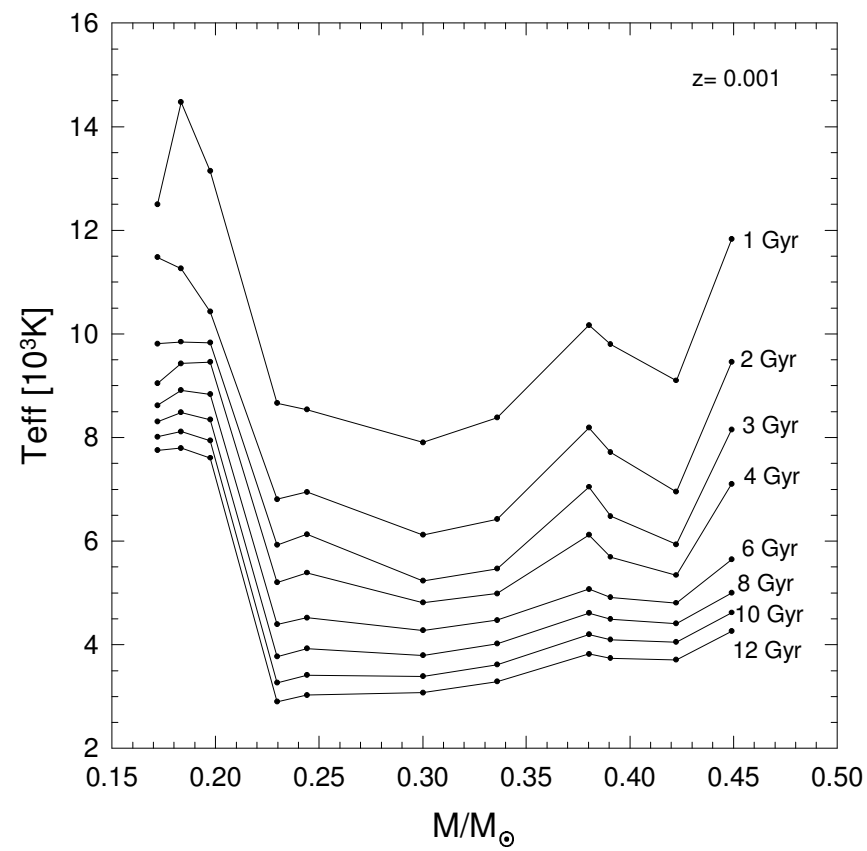

Figura 5.8: Isocronas para nuestras secuencias de HeWDs de $Z=0.001$. La caída abrupta que muestran en el rango $0.20-0.22 \mathrm{M}_{\odot}$ es consecuencia de la existencia de una masa límite para la ocurrencia de los flashes de CNO. Edades mucho más cortas resultan si la masa del modelo es mayor que la de la masa límite. El máximo local en $\mathrm{T}_{\text {eff }}$ que se exhibe en $\approx 0.380 \mathrm{M}_{\odot}$ está relacionado con el aumento en la eficiencia de la combustión nuclear estable vía PP a edades bajas.

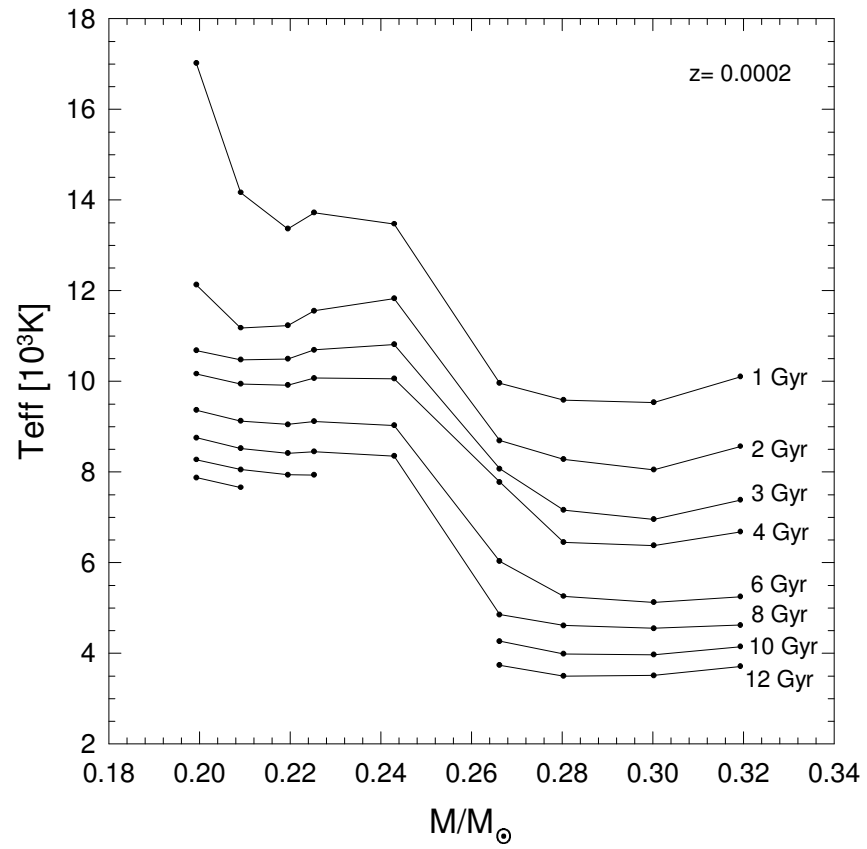

Figura 5.9: Igual que en la figura 5.8 pero para el caso de $Z=0.0002$. Aquí las isocronas no exhiben una caída tan pronunciada como en el caso de los modelos de $Z=0.001$. Para los modelos que no sufren flashes de CNO, los cálculos fueron detenidos antes de que los flashes de PP cominecen y por lo tanto, algunos modelos de masas bajas no han alcanzado los $10 \mathrm{Gyr}$ de edad. 

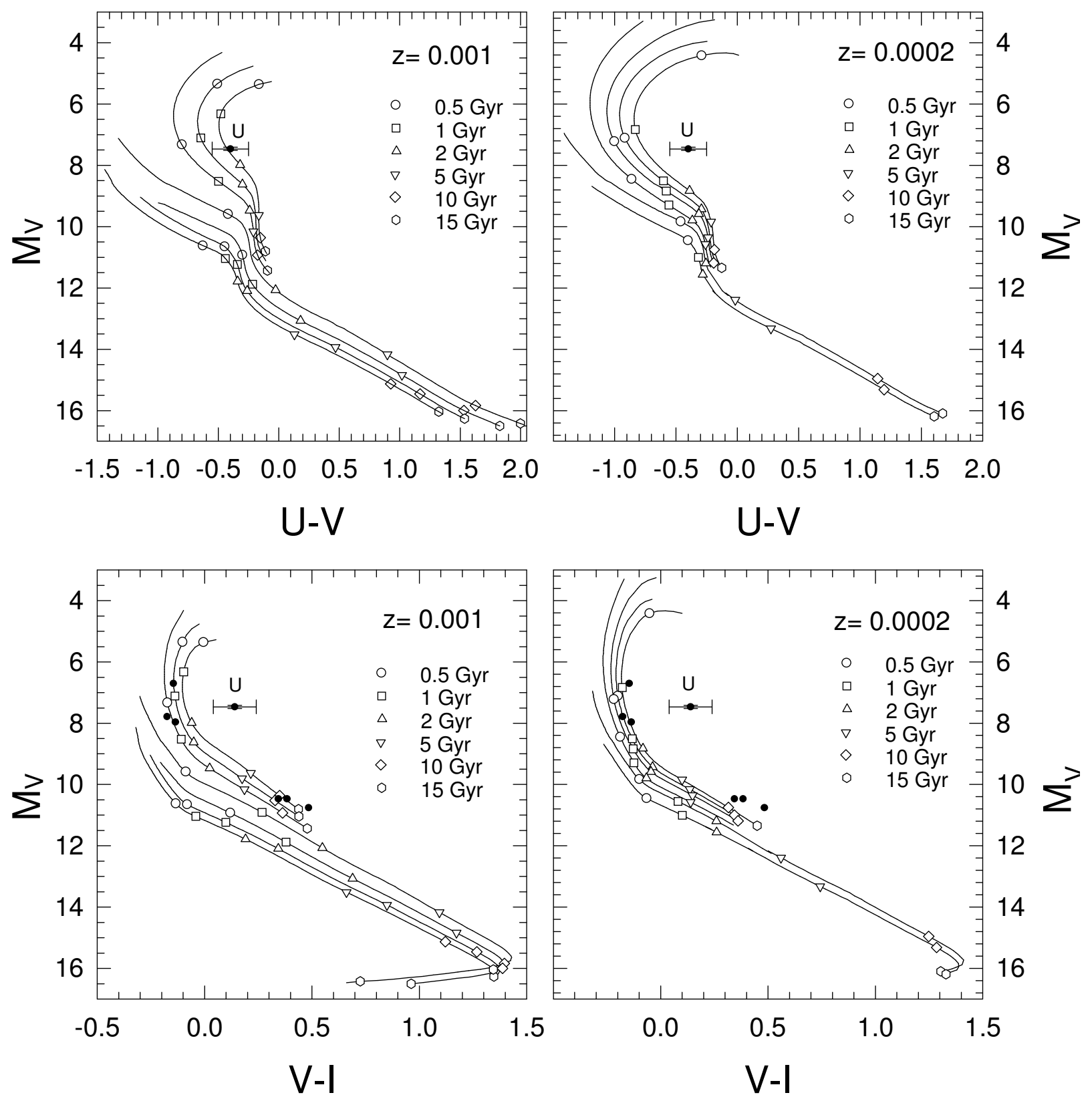

Figura 5.10: Magnitud visual absoluta en términos de los índices de color $U-V$ (paneles superiores) y $V-I$ (paneles inferiores) para los modelos de HeWD de $0.172,0.183,0.197,0.230,0.300,0.380$ y $0.449 \mathrm{M}_{\odot}$ en el caso de $Z=0.001$, y $0.199,0.209,0.219,0.243,0.266$ y $0.319 \mathrm{M}_{\odot}$ en el de $Z=0.0002$. Los símbolos a lo largo de las curvas denotan las edades de enfriamiento. Además, se incluyen los datos observacionales para la HeWD in 47 Tucanae (Edmonds et al., 2001, denotada "U") y las seis candidatas a HeWD en NGC 6397 reportadas por Taylor et al. (2001, círculos llenos). 
los modelos permanecen más brillantes que $M_{V} \approx 17$ durante las etapas posteriores de enfriamiento. Un comportamiento análogo fue encontrado para modelos de HeWD de metalicidad solar (capítulo 4), aunque en ese caso las HeWDs de hasta $\approx 0.19 \mathrm{M}_{\odot}$ alcanzan valores de la magnitud tan grandes.

En la figura 5.10 también hemos incluido las observaciones recientes de Taylor et al. (2001) y Edmonds et al. (2001) de las candidatas a HeWDs detectadas en NGC 6397 y 47 Tucanae, respectivamente. Para NGC 6397 hemos adoptado el módulo de distancia $(m-M)_{V}=12.29 \mathrm{y}$ un enrojecimiento $E(B-V)=0.18$ (Cool et al., 1998). En el caso de 47 Tucanae fueron usados los valores $(m-M)_{V}=13.39$ y $E(B-V)=0.055$ (Zoccali et al., 2001) para transformar a magnitudes absolutas y para desenrojecer los índices de color. En ambos casos, se aplicaron las leyes de extinción de Holtzman et al. (1995). Consideremos primero las candidatas a HeWD de NGC 6397. Dada la metalicidad de este CG (Fe/H= -1.82]; Anthony-Twarog \& Twarog 2000), nuestras secuencias de $Z=0.0002$ son apropiadas para una comparación directa con las observaciones. Notar que las seis candidatas pueden separarse en dos grupos de tres estrellas cada uno. En particular, para las tres estrellas más brillantes nuestros modelos evolutivos ajustan muy bien la secuencia observada. Encontramos que tales estrellas, en caso de ser HeWDs, estarían caracterizadas por valores de masa estelar entre 0.20 y $0.22 \mathrm{M}_{\odot}$, que está por debajo del valor $M_{\text {th }}$ para la ocurrencia de flashes CNO. Con respecto a la determincación de sus edades, encontramos que tienen entre 0.5 y 1.5 Gyr. En contraste, las tres estrellas menos brillantes parecen tener radios mayores incluso que nuestro modelo menos masivo $\left(\approx 0.20 \mathrm{M}_{\odot}\right)$, como resulta claro de la figura 5.10. Queremos señalar que en base al algoritmo de pérdida de masa que hemos implementado encontramos un valor mínimo posible para la HeWD remanente cercano a $0.2 \mathrm{M}_{\odot}$ para progenitores con $Z=0.0002$. Para revisar este punto, hemos realizado cálculos adicionales de modelos pre-WD variando la configuración inicial del sistema binario y en ningún caso hemos obtenido HeWD remanentes menos masivas que $0.195 \mathrm{M}_{\odot}$. Se han realizado pruebas también cambiando el esquema de pérdida de masa, adoptando el propuesto por Ritter (1988) y el resultado encontrado ha sido el mismo. En este contexto, es importante destacar que Burderi, D'Antona \& Burgay (2002) encuentran también una masa mínima límite para el objeto remanente cercana $a \approx 0.2 \mathrm{M}_{\odot}$. Burderi, D'Antona \& Burgay (2002) también encuentran que sus modelos tienen radios demasiado pequeños para ajustar estas observaciones. Sin embargo, hay que mencionar que nuestros modelos son menos compactos que los de Burderi, D'Antona \& Burgay (2002). Creemos que la diferencia puede deberse a que en nuestros modelos la sedimentación graviatoria lleva a la formación de envolturas de hidrógeno puro (ver el capítulo 3 en relación a cómo la sedimentación gravitatoria afecta las relaciones masa-radio), mientras que este efecto parece no estar considerado en los cálculos de Burderi, D’Antona \& Burgay (2002). Por este motivo, la discrepancia entre las observaciones y los cálculos teóricos es menor en el caso de nuestros modelos evolutivos. Otro punto importante en relación con estas tres estrellas son las estimaciones de sus edades. Nuestra secuencia menos masiva da para estas estrellas edades entre 10 y 15 Gyr. Si tomamos en cuenta el tiempo transcurrido durante la evolución pre-WD (que es de entre 6 y 7 Gyr para un progenitor de $1 \mathrm{M}_{\odot}$ ), entonces la edad de estas estrellas estaría entre 16 y $21 \mathrm{Gyr}$, mayor que la edad del cúmulo, que es $12 \pm 0.8$ Gyr (Anthony-Twarog $\&$ Twarog, 2000). Esto sugiere que los progenitores de estas candidatas a HeWD deberían haber sido más masivos que $1 \mathrm{M}_{\odot}$, para tener edades evolutivas pre-WD más cortas. Para explorar esta posibilidad, hemos realizado cálculos adicionales con nuestra prescripción para la pérdida de masa durante la evolución binaria. Hemos supuesto que el progenitor de HeWD tiene $1.4 \mathrm{M}_{\odot}$ y una compañera de la misma masa, y un período inicial de 0.5 días. En este caso encontramos que la evolución pre-WD dura unos 3 Gyr y la masa de la HeWD remanente 
tiene $0.195 \mathrm{M}_{\odot}$. Esta posibilidad alivia la discrepancia entre las edades del cúmulo y las tres candidatas a HeWD más débiles en NGC 6397. En este sentido, un estudio extensivo de la naturaleza de los sistemas binarios que llevan a la formación de HeWDs, particularmente para progenitores de baja metalicidad, es necesario para presentar conclusiones al respecto.

Con relación a la candidata a HeWD en el CG 47 Tucanae, encontramos que nuestras secuencias evolutivas con $Z=0.001$, un valor de metalicidad no muy diferente de aquél del cúmulo ([Fe/H=-0.76]), dan un buen acuerdo con las observaciones para el índice de color $U-V$. en particular, derivamos para la WD una masa de $\approx 0.17 \mathrm{M}_{\odot}$ y una edad de enfriamiento alrededor de 1.5 Gyr. Esta edad es consistente con los 2 Gyr correspondientes a la edad de spin-down del púlsar de milisegundo del cual esta WD es compañera (ver Edmonds et al. 2001 y referencias en ese artículo). Notar que, en lo que al índice $V-I$ respecta, no encontramos acuerdo entre nuestras secuencias y la observación. En efecto, nuestros modelos aparecen demasiado lejos hacia el azul. Un valor menor de la masa $\left(\approx 0.15-0.16 \mathrm{M}_{\odot}\right)$ sería necesario para obtener acuerdo con la observación del índice $V-I$. En el caso de $Z=0.0002$ y para las masas estelares cubiertas por nuestras secuencias no encontramos acuerdo para ninguna de las masas.

Finalmente, en las tablas 5.3 y 5.2 listamos las características principales de algunos modelos seleccionados para nuestras secuencias de HeWD de $Z=0.0002$ y $Z=0.001$, respectivamente.

Table 5.2: Modelos seleccionados de nuestras secuencias de $0.199,0.243$ y $0.300 \mathrm{M}_{\odot}$ para $Z=0.0002$.

\begin{tabular}{|c|c|c|c|c|c|c|c|c|c|c|c|c|}
\hline $\bar{M}_{*} / \mathrm{M}_{\odot}$ & $\mathrm{T}_{\text {eff }}$ & $\log (g)$ & $\operatorname{Edad}(\mathrm{Gyr})$ & U-B & B-V & V-R & V-K & R-I & $\mathrm{J}-\mathrm{H}$ & H-K & $\mathrm{BC}$ & $\mathrm{M}_{V}$ \\
\hline 0.199 & 8920 & 4.3218 & 0.295 & -0.09 & 0.10 & 0.04 & 0.12 & 0.06 & 0.07 & -0.09 & -0.09 & 4.41 \\
\hline 0.199 & 10780 & 4.5281 & 0.494 & -0.24 & -0.05 & -0.03 & -0.20 & -0.02 & 0.02 & -0.10 & -0.40 & 4.42 \\
\hline 0.199 & 14220 & 5.0085 & 0.683 & -0.52 & -0.13 & -0.07 & -0.45 & -0.07 & -0.02 & -0.12 & -1.08 & 5.09 \\
\hline 0.199 & 16150 & 5.3500 & 0.791 & -0.64 & -0.15 & -0.08 & -0.54 & -0.09 & -0.04 & -0.13 & -1.41 & 5.72 \\
\hline 0.199 & 16880 & 5.5465 & 0.861 & -0.68 & -0.16 & -0.08 & -0.57 & -0.10 & -0.04 & -0.13 & -1.52 & 6.14 \\
\hline 0.199 & 17110 & 5.6901 & 0.924 & -0.69 & -0.15 & -0.08 & -0.58 & -0.10 & -0.04 & -0.13 & -1.56 & 6.47 \\
\hline 0.199 & 16810 & 5.9008 & 1.054 & -0.68 & -0.14 & -0.08 & -0.56 & -0.10 & -0.04 & -0.13 & -1.51 & 7.03 \\
\hline 0.199 & 16430 & 5.9817 & 1.124 & -0.66 & -0.13 & -0.08 & -0.55 & -0.09 & -0.04 & -0.13 & -1.45 & 7.27 \\
\hline 0.199 & 15960 & 6.0522 & 1.201 & -0.64 & -0.12 & -0.08 & -0.53 & -0.09 & -0.03 & -0.13 & -1.37 & 7.49 \\
\hline 0.199 & 15430 & 6.1141 & 1.283 & -0.61 & -0.11 & -0.07 & -0.50 & -0.08 & -0.03 & -0.12 & -1.28 & 7.71 \\
\hline 0.199 & 14870 & 6.1704 & 1.375 & -0.58 & -0.09 & -0.07 & -0.47 & -0.08 & -0.02 & -0.12 & -1.19 & 7.91 \\
\hline 0.199 & 14280 & 6.2219 & 1.478 & -0.54 & -0.08 & -0.07 & -0.45 & -0.07 & -0.02 & -0.12 & -1.08 & 8.11 \\
\hline 0.199 & 13700 & 6.2699 & 1.594 & -0.51 & -0.06 & -0.06 & -0.41 & -0.06 & -0.01 & -0.12 & -0.97 & 8.30 \\
\hline 0.199 & 13110 & 6.3151 & 1.725 & -0.47 & -0.04 & -0.06 & -0.38 & -0.06 & -0.01 & -0.12 & -0.86 & 8.49 \\
\hline 0.199 & 12530 & 6.3582 & 1.876 & -0.43 & -0.01 & -0.05 & -0.34 & -0.05 & 0.00 & -0.12 & -0.74 & 8.68 \\
\hline 0.199 & 11960 & 6.4003 & 2.058 & -0.39 & 0.02 & -0.04 & -0.29 & -0.04 & 0.01 & -0.11 & -0.63 & 8.88 \\
\hline 0.199 & 11430 & 6.4419 & 2.295 & -0.36 & 0.05 & -0.03 & -0.24 & -0.02 & 0.02 & -0.11 & -0.53 & 9.07 \\
\hline 0.199 & 10930 & 6.4858 & 2.669 & -0.35 & 0.08 & -0.02 & -0.18 & -0.01 & 0.03 & -0.11 & -0.43 & 9.28 \\
\hline 0.199 & 10470 & 6.5314 & 3.356 & -0.35 & 0.11 & 0.00 & -0.12 & 0.01 & 0.03 & -0.11 & -0.34 & 9.49 \\
\hline 0.199 & 9990 & 6.5726 & 4.351 & -0.37 & 0.15 & 0.03 & -0.04 & 0.04 & 0.05 & -0.10 & -0.26 & 9.72 \\
\hline 0.199 & 9510 & 6.6103 & 5.577 & -0.39 & 0.18 & 0.06 & 0.08 & 0.07 & 0.06 & -0.10 & -0.21 & 9.98 \\
\hline 0.199 & 9040 & 6.6448 & 6.985 & -0.42 & 0.22 & 0.10 & 0.21 & 0.10 & 0.08 & -0.09 & -0.17 & 10.24 \\
\hline 0.199 & 8570 & 6.6772 & 8.658 & -0.45 & 0.26 & 0.13 & 0.34 & 0.13 & 0.10 & -0.08 & -0.14 & 10.53 \\
\hline
\end{tabular}


Continúa tabla página anterior

\begin{tabular}{|c|c|c|c|c|c|c|c|c|c|c|c|c|}
\hline$M_{*} / \mathrm{M}_{\odot}$ & $\mathrm{T}_{\mathrm{eff}}$ & $\log (g)$ & $E d a d(\mathrm{Gyr})$ & U-B & $\mathrm{B}-\mathrm{V}$ & V-R & V-K & R-I & $\mathrm{J}-\mathrm{H}$ & H-K & $\mathrm{BC}$ & $\mathrm{M}_{V}$ \\
\hline 0.199 & 8110 & 6.7069 & 10.622 & -0.47 & 0.29 & 0.17 & 0.49 & 0.17 & 0.13 & -0.07 & -0.13 & 10.82 \\
\hline 0.199 & 250 & 6.7575 & 15.738 & -0.48 & 0.36 & 0.23 & 0.81 & 0.23 & 0.18 & -0.04 & -0.11 & 11.42 \\
\hline 0.243 & 15050 & 4.4210 & 0.131 & -0.59 & -0.18 & -0.08 & -0.51 & -0.09 & -0.03 & -0.12 & -1.21 & 3.29 \\
\hline 0.243 & 21020 & 5.0959 & 0.161 & -0.84 & -0.23 & -0.10 & -0.72 & -0.13 & -0.07 & -0.14 & -2.10 & 4.42 \\
\hline 0.243 & 23930 & 5.4719 & 0.178 & -0.93 & -0.24 & -0.11 & -0.80 & -0.14 & -0.08 & -0.15 & -2.44 & 5.13 \\
\hline 0.243 & 25070 & 5.7041 & 0.190 & -0.95 & -0.24 & -0.12 & -0.82 & -0.15 & -0.08 & -0.15 & -2.56 & 5.63 \\
\hline 0.243 & 25360 & 5.8768 & 0.201 & -0.96 & -0.24 & -0.12 & -0.83 & -0.15 & -0.09 & -0.16 & -2.59 & 6.04 \\
\hline 0.243 & 24650 & 6.1290 & 0.229 & -0.94 & -0.23 & -0.12 & -0.81 & -0.14 & -0.08 & -0.15 & -2.51 & 6.72 \\
\hline 0.243 & 23920 & 6.2282 & 0.250 & -0.93 & -0.22 & -0.11 & -0.79 & -0.14 & -0.08 & -0.15 & -2.43 & 7.02 \\
\hline 0.243 & 23020 & 6.3141 & 0.277 & -0.91 & -0.21 & -0.11 & -0.77 & -0.14 & -0.07 & -0.15 & -2.33 & 7.30 \\
\hline 0.243 & 2000 & 6.3880 & & -0.88 & -0.19 & -0.10 & -0.74 & -0.13 & -0.07 & -0.15 & -2.21 & 7.56 \\
\hline 0.243 & 20900 & 6.4526 & & -0.85 & -0.18 & -0.10 & -0.71 & -0.12 & -0.06 & -0.14 & -2.08 & 7.81 \\
\hline 0.243 & 9780 & 6.5089 & & -0.81 & -0.16 & -0.10 & -0.67 & -0.12 & -0.06 & -0.14 & -1.93 & 8.05 \\
\hline 0.243 & 3650 & 6.5594 & & -0.77 & -0.14 & -0.09 & -0.64 & -0.11 & -0.05 & -0.14 & -1.78 & 8.27 \\
\hline 0.243 & 17540 & 6.6058 & & -0.72 & -0.12 & -0.09 & -0.59 & -0.10 & -0.04 & -0.13 & -1.61 & 8.49 \\
\hline 0.243 & 460 & 6489 & & .68 & -0.10 & -0.08 & .55 & -0.09 & -0.04 & -0.13 & -1.44 & 8.71 \\
\hline 0.243 & 15420 & 6.6900 & & -0.63 & -0.08 & -0.07 & -0.51 & -0.08 & -0.03 & -0.13 & -1.27 & 8.92 \\
\hline 0.243 & 14450 & 6.7296 & 0.826 & -0.57 & -0.05 & -0.07 & -0.46 & -0.07 & -0.02 & -0.12 & -1.10 & 9.13 \\
\hline 243 & 540 & 7702 & & -0.52 & -0.02 & -0.06 & -0.41 & -0.06 & -0.01 & -0.12 & -0.93 & 9.34 \\
\hline 0.243 & 2700 & 6.8121 & & -0.46 & 0.01 & -0.05 & -0.35 & -0.05 & 0.00 & -0.12 & -0.77 & 9.57 \\
\hline 0.243 & 870 & 6.8494 & 99 & -0.42 & 0.05 & -0.04 & -0.28 & -0.03 & 0.01 & -0.11 & -0.61 & 9.79 \\
\hline 0.243 & 060 & 6.8826 & & -0.41 & 0.09 & -0.01 & -0.20 & 0.00 & 0.03 & -0.11 & -0.45 & 10.02 \\
\hline 243 & 290 & 9114 & & -0.42 & 0.14 & 0.02 & -0.08 & 0.03 & 0.04 & -0.11 & -0.31 & 10.27 \\
\hline 0.243 & 9560 & 9384 & & -0.44 & 0.18 & 0.07 & 0.09 & 0.07 & 0.06 & -0.10 & -0.23 & 10.58 \\
\hline 0.243 & 8240 & 6.9865 & & -0.49 & 0.26 & 0.16 & 0.46 & 0.16 & 0.12 & -0.07 & -0.14 & 11.25 \\
\hline 300 & 990 & 9774 & & -0.97 & -0.21 & -0.12 & -0.82 & -0.15 & -0.08 & -0.16 & -2.53 & 8.57 \\
\hline 0.300 & 390 & 9941 & & -0.93 & -0.19 & -0.11 & -0.78 & -0.14 & -0.07 & -0.15 & -2.36 & 8.73 \\
\hline 0.300 & 800 & 7.0043 & 0.076 & -0.89 & -0.17 & -0.10 & -0.74 & -0.13 & -0.07 & -0.15 & -2.18 & 8.88 \\
\hline 300 & 050 & 7.0178 & & -0.83 & -0.15 & -0.10 & -0.68 & -0.12 & -0.06 & -0.14 & -1.96 & 9.06 \\
\hline 0.300 & 730 & .0331 & & -0.79 & -0.13 & -0.09 & -0.64 & -0.11 & -0.05 & -0.14 & -1.78 & 9.21 \\
\hline 0.300 & 510 & 7.0495 & 0.162 & -0.74 & -0.10 & -0.09 & -0.60 & -0.10 & -0.04 & -0.13 & -1.60 & 9.36 \\
\hline 0.300 & 160 & 7.0699 & & -0.68 & -0.08 & -0.08 & -0.54 & -0.09 & -0.03 & -0.13 & -1.39 & 9.55 \\
\hline 0.300 & 14930 & 7.0903 & & -0.62 & -0.05 & -0.07 & -0.48 & -0.07 & -0.02 & -0.12 & -1.18 & 9.73 \\
\hline 0.300 & 770 & 7.1113 & & -0.55 & -0.01 & -0.06 & -0.42 & -0.06 & -0.01 & -0.12 & -0.97 & 9.93 \\
\hline 0.300 & 870 & 7.1293 & & -0.50 & 0.03 & -0.05 & -0.36 & -0.05 & 0.00 & -0.12 & -0.80 & 10.10 \\
\hline 0.300 & 12050 & 7.1470 & 0.424 & -0.46 & 0.06 & -0.04 & -0.29 & -0.03 & 0.01 & -0.12 & -0.64 & 10.27 \\
\hline 0.300 & 110 & 7.1691 & & -0.45 & 0.10 & -0.01 & -0.20 & 0.00 & 0.02 & -0.11 & -0.45 & 10.4 \\
\hline 0.300 & 10270 & 7.1897 & & -0.46 & 0.15 & 0.03 & -0.06 & 0.04 & 0.04 & -0.11 & -0.32 & 10.7 \\
\hline 0.300 & 9470 & 7.2094 & 1.024 & -0.48 & 0.19 & 0.08 & 0.12 & 0.08 & 0.07 & -0.09 & -0.23 & 11.06 \\
\hline 0.300 & 8870 & 7.2239 & 1.336 & -0.50 & 0.22 & 0.12 & 0.28 & 0.12 & 0.09 & -0.08 & -0.19 & 11.3 \\
\hline $300(\mathrm{~A})$ & 8290 & 7.2368 & 1.766 & -0.51 & 0.26 & 0.16 & 0.46 & 0.16 & 0.12 & -0.07 & -0.15 & 11.6 \\
\hline $.300(\mathrm{~B})$ & 7800 & 7.2634 & 2.776 & -0.51 & 0.30 & 0.19 & 0.61 & 0.20 & 0.15 & -0.06 & -0.13 & 11.94 \\
\hline 0.300 & 7150 & 7.2688 & 2.796 & -0.48 & 0.37 & 0.24 & 0.85 & 0.25 & 0.19 & -0.03 & -0.12 & 10.3 \\
\hline
\end{tabular}


Continúa tabla página anterior

\begin{tabular}{ccccccccccccc}
\hline$M_{*} / \mathrm{M}_{\odot}$ & $\mathrm{T}_{\text {eff }}$ & $\log (g)$ & $E d a d(\mathrm{Gyr})$ & $\mathrm{U}-\mathrm{B}$ & $\mathrm{B}-\mathrm{V}$ & $\mathrm{V}-\mathrm{R}$ & $\mathrm{V}-\mathrm{K}$ & $\mathrm{R}-\mathrm{I}$ & $\mathrm{J}-\mathrm{H}$ & $\mathrm{H}-\mathrm{K}$ & $\mathrm{BC}$ & $\mathrm{M}_{V}$ \\
\hline & & & & & & & & & & & & \\
0.300 & 6570 & 7.2731 & 3.751 & -0.42 & 0.44 & 0.29 & 1.09 & 0.30 & 0.23 & -0.01 & -0.11 & 12.69 \\
0.300 & 6060 & 7.2852 & 4.394 & -0.33 & 0.53 & 0.35 & 1.33 & 0.35 & 0.27 & 0.01 & -0.12 & 13.08 \\
0.300 & 5210 & 7.3257 & 5.748 & -0.11 & 0.72 & 0.47 & 1.82 & 0.47 & 0.32 & 0.07 & -0.21 & 13.93 \\
0.300 & 4840 & 7.3486 & 6.969 & 0.00 & 0.82 & 0.53 & 2.07 & 0.53 & 0.35 & 0.09 & -0.31 & 14.40 \\
0.300 & 4470 & 7.3622 & 8.269 & 0.12 & 0.92 & 0.59 & 2.23 & 0.59 & 0.33 & 0.04 & -0.42 & 14.89 \\
0.300 & 4110 & 7.3696 & 9.479 & 0.22 & 1.01 & 0.65 & 2.12 & 0.65 & 0.12 & -0.02 & -0.46 & 15.32 \\
0.300 & 3770 & 7.3743 & 10.729 & 0.29 & 1.07 & 0.69 & 1.67 & 0.68 & -0.14 & -0.14 & -0.41 & 15.65 \\
0.300 & 3460 & 7.3785 & 12.260 & 0.36 & 1.13 & 0.72 & 1.12 & 0.68 & -0.30 & -0.28 & -0.31 & 15.93 \\
0.300 & 3180 & 7.3835 & 15.016 & 0.44 & 1.19 & 0.72 & 0.59 & 0.60 & -0.37 & -0.36 & -0.16 & 16.16 \\
0.300 & 2920 & 7.3858 & 16.606 & 0.51 & 1.24 & 0.69 & 0.05 & 0.43 & -0.38 & -0.47 & 0.01 & 16.37 \\
\hline
\end{tabular}

Las edades se cuentan desde el fin de la transferencia de masa. Las letras A y B denotan el intervalo durante el cual el modelo de $0.300 \mathrm{M}_{\odot}$ retorna varias veces a altos valores de $\mathrm{T}_{\text {eff }}$ como resultado de los flashes PP.

Table 5.3: Modelos seleccionados de nuestras secuencias de $0.172,0.230,0.336$ y $0.449 \mathrm{M}_{\odot}$ para $Z=0.001$.

\begin{tabular}{|c|c|c|c|c|c|c|c|c|c|c|c|c|}
\hline$M_{*} / \mathrm{M}_{\odot}$ & $\mathrm{T}_{\mathrm{eff}}$ & $\log (g)$ & $E d a d(\mathrm{Gyr})$ & U-B & $\mathrm{B}-\mathrm{V}$ & V-R & V-K & R-I & $\mathrm{J}-\mathrm{H}$ & H-K & $\mathrm{BC}$ & $\mathrm{M}_{V}$ \\
\hline 0.172 & 9390 & 4.6630 & 0.349 & -0.13 & 0.07 & 0.02 & 0.02 & 0.03 & 0.05 & -0.09 & -0.16 & 5.27 \\
\hline 0.172 & 12420 & 5.2994 & 0.973 & -0.40 & -0.07 & -0.05 & -0.33 & -0.05 & 0.00 & -0.11 & -0.74 & 6.22 \\
\hline 0.172 & 12760 & 5.4971 & 1.173 & -0.42 & -0.07 & -0.05 & -0.35 & -0.05 & 0.00 & -0.11 & -0.81 & 6.67 \\
\hline 0.172 & 12700 & 5.6392 & 1.356 & -0.42 & -0.06 & -0.05 & -0.35 & -0.05 & 0.00 & -0.11 & -0.79 & 7.03 \\
\hline 0.172 & 12410 & 5.7509 & 1.544 & -0.40 & -0.05 & -0.05 & -0.33 & -0.05 & 0.00 & -0.11 & -0.73 & 7.35 \\
\hline 0.172 & 12010 & 5.8440 & 1.746 & -0.37 & -0.03 & -0.04 & -0.30 & -0.04 & 0.01 & -0.11 & -0.65 & 7.64 \\
\hline 0.172 & 11540 & 5.9253 & 1.971 & -0.33 & 0.00 & -0.04 & -0.26 & -0.03 & 0.01 & -0.11 & -0.56 & 7.93 \\
\hline 0.172 & 11040 & 5.9987 & 2.223 & -0.30 & 0.04 & -0.03 & -0.20 & -0.02 & 0.02 & -0.11 & -0.46 & 8.21 \\
\hline 0.172 & 10520 & 6.0664 & 2.511 & -0.29 & 0.07 & -0.01 & -0.14 & 0.00 & 0.03 & -0.10 & -0.36 & 8.48 \\
\hline 0.172 & 10000 & 6.1303 & 2.849 & -0.30 & 0.12 & 0.02 & -0.06 & 0.03 & 0.04 & -0.10 & -0.26 & 8.77 \\
\hline 0.172 & 9500 & 6.1927 & 3.279 & -0.33 & 0.16 & 0.05 & 0.06 & 0.06 & 0.06 & -0.10 & -0.20 & 9.08 \\
\hline 0.172 & 9050 & 6.2590 & 3.974 & -0.36 & 0.19 & 0.09 & 0.18 & 0.09 & 0.07 & -0.09 & -0.16 & 9.42 \\
\hline 0.172 & 8660 & 6.3336 & 5.723 & -0.39 & 0.22 & 0.12 & 0.30 & 0.12 & 0.09 & -0.08 & -0.14 & 9.78 \\
\hline 0.172 & 8230 & 6.3987 & 8.461 & -0.42 & 0.26 & 0.15 & 0.44 & 0.15 & 0.12 & -0.07 & -0.12 & 10.14 \\
\hline 0.172 & 7330 & 6.5018 & 15.655 & -0.45 & 0.34 & 0.23 & 0.77 & 0.23 & 0.17 & -0.04 & -0.10 & 10.88 \\
\hline 0.172 & 6880 & 6.5439 & 20.326 & -0.42 & 0.40 & 0.26 & 0.95 & 0.27 & 0.20 & -0.02 & -0.09 & 11.26 \\
\hline 0.230 & 30010 & 6.4188 & 0.203 & -1.06 & -0.27 & -0.13 & -0.94 & -0.17 & -0.10 & -0.17 & -2.98 & 7.12 \\
\hline 0.230 & 28220 & 6.4678 & 0.203 & -1.02 & -0.25 & -0.13 & -0.90 & -0.16 & -0.10 & -0.17 & -2.84 & 7.37 \\
\hline 0.230 & 26430 & 6.5134 & 0.203 & -0.99 & -0.23 & -0.12 & -0.86 & -0.15 & -0.09 & -0.16 & -2.68 & 7.61 \\
\hline 0.230 & 25000 & 6.5480 & 0.203 & -0.96 & -0.22 & -0.12 & -0.82 & -0.15 & -0.08 & -0.15 & -2.54 & 7.80 \\
\hline 0.230 & 23350 & 6.5868 & 0.203 & -0.92 & -0.20 & -0.11 & -0.78 & -0.14 & -0.07 & -0.15 & -2.36 & 8.02 \\
\hline 0.230 & 22030 & 6.6168 & 0.204 & -0.89 & -0.19 & -0.10 & -0.74 & -0.13 & -0.07 & -0.15 & -2.21 & 8.19 \\
\hline 0.230 & 20710 & 6.6461 & 0.205 & -0.85 & -0.17 & -0.10 & -0.70 & -0.12 & -0.06 & -0.14 & -2.05 & 8.37 \\
\hline
\end{tabular}


Continúa tabla página anterior

\begin{tabular}{|c|c|c|c|c|c|c|c|c|c|c|c|c|}
\hline$M_{*} / \mathrm{M}_{\odot}$ & $\mathrm{T}_{\text {eff }}$ & $\log (g)$ & $\operatorname{Edad}(\mathrm{Gyr})$ & U-B & B-V & V-R & V-K & R-I & $\mathrm{J}-\mathrm{H}$ & H-K & $\mathrm{BC}$ & $\mathrm{M}_{V}$ \\
\hline 0.230 & 19200 & 6.6727 & 0.214 & -0.79 & -0.15 & -0.09 & -0.65 & -0.11 & -0.05 & -0.14 & -1.85 & 8.56 \\
\hline 0.230 & 17860 & 6.6782 & 0.251 & -0.74 & -0.13 & -0.09 & -0.61 & -0.10 & -0.04 & -0.13 & -1.66 & 8.70 \\
\hline 0.230 & 16620 & 6.6907 & 0.299 & -0.69 & -0.10 & -0.08 & -0.56 & -0.09 & -0.04 & -0.13 & -1.47 & 8.85 \\
\hline 0.230 & 15380 & 6.7116 & & -0.62 & -0.08 & -0.07 & -0.50 & -0.08 & -0.03 & -0.13 & -1.26 & 9.04 \\
\hline 0.230 & 14310 & 6.7331 & 0.390 & -0.56 & -0.05 & -0.07 & -0.45 & -0.07 & -0.02 & -0.12 & -1.07 & 9.21 \\
\hline 0.230 & 13320 & 6.7549 & 0.442 & -0.50 & -0.02 & -0.06 & -0.39 & -0.06 & -0.01 & -0.12 & -0.89 & 9.40 \\
\hline 0.230 & 12400 & 6.7768 & & -0.44 & 0.02 & -0.05 & -0.33 & -0.04 & 0.01 & -0.12 & -0.71 & 9.59 \\
\hline 0.230 & 11530 & 6.7990 & & -0.40 & 0.06 & -0.03 & -0.25 & -0.02 & 0.02 & -0.11 & -0.55 & 9.79 \\
\hline 0.230 & 10730 & 6.8210 & & -0.40 & 0.11 & 0.00 & -0.15 & 0.01 & 0.03 & -0.11 & -0.38 & 9.99 \\
\hline 0.230 & 9910 & 6.8455 & & -0.41 & 0.15 & 0.04 & 0.00 & 0.05 & 0.05 & -0.10 & -0.26 & 10.28 \\
\hline 0.230 & 9220 & 6.8699 & 0.868 & -0.44 & 0.20 & 0.09 & 0.17 & 0.09 & 0.07 & -0.09 & -0.20 & 10.59 \\
\hline 0.230 & 8600 & 8939 & & -0.47 & 0.24 & 0.13 & .35 & 0.14 & 0.10 & -0.08 & -0.16 & 10.91 \\
\hline 0.230 & 8070 & 6.9151 & & -0.48 & 0.28 & 0.17 & 0.51 & 0.17 & 0.13 & -0.06 & -0.13 & 11.21 \\
\hline 0.230 & 7000 & 9588 & & -0.46 & 0.38 & 0.25 & 0.90 & 0.26 & 0.20 & -0.03 & -0.10 & 11.91 \\
\hline 0.230 & 6460 & 9796 & & -0.40 & 0.45 & 0.30 & 1.13 & 0.30 & 0.24 & -0.01 & -0.10 & 12.31 \\
\hline 0.230 & 5970 & 9984 & & -0.31 & 0.54 & 0.36 & 1.37 & 0.36 & 0.27 & 0.02 & -0.12 & 12.71 \\
\hline 0.230 & 50 & 0477 & & -0.09 & 0.74 & 0.47 & 1.86 & 0.47 & 0.32 & .08 & -0.23 & 13.59 \\
\hline 0.230 & 4810 & 7.0820 & & 0.02 & 0.83 & 0.54 & 2.11 & 0.53 & 0.35 & 0.10 & -0.32 & 14.07 \\
\hline 0.230 & 4460 & 1098 & & 0.14 & 0.93 & 0.60 & 2.29 & 0.60 & 0.35 & 0.06 & -0.43 & 14.58 \\
\hline 0.230 & 4110 & 7.1265 & & 0.24 & 1.02 & 0.66 & 2.23 & 0.66 & 0.18 & 0.00 & -0.49 & 15.04 \\
\hline 0.230 & 80 & 1369 & & 32 & 1.09 & 0.70 & 1.85 & 0.69 & -0.08 & -0.11 & -0.46 & 15.39 \\
\hline 0.230 & 3450 & 1443 & & 39 & 1.15 & 0.73 & 1.25 & 0.69 & -0.27 & -0.26 & -0.35 & 15.69 \\
\hline 0.230 & 3210 & 7.1486 & & 0.46 & 1.20 & 0.74 & 0.74 & 0.64 & -0.36 & -0.35 & -0.22 & 15.89 \\
\hline 0.230 & 2980 & 1522 & & 0.52 & 1.25 & 0.72 & 0.27 & 0.51 & -0.38 & -0.43 & -0.07 & 16.07 \\
\hline 0.230 & 2760 & 1552 & & 0.59 & 1.29 & 0.69 & -0.16 & 0.33 & -0.36 & -0.54 & 0.08 & 16.26 \\
\hline 0.230 & 2530 & 1593 & & 0.68 & 1.35 & 0.62 & -0.70 & 0.04 & -0.29 & -0.69 & 0.26 & 16.46 \\
\hline 0.230 & 2310 & 7.1624 & 18.216 & 0.78 & 1.40 & 0.55 & -1.27 & -0.34 & -0.20 & -0.87 & 0.45 & 16.69 \\
\hline 0.336 & 420 & 1810 & & -0.88 & -0.16 & -0.10 & -0.73 & -0.12 & -0.06 & -0.14 & -2.13 & 9.22 \\
\hline 0.336 & 19620 & 7.1907 & & -0.82 & -0.13 & -0.10 & -0.67 & -0.11 & -0.05 & -0.14 & -1.90 & 9.40 \\
\hline 0.336 & 18260 & 7.1978 & & -0.78 & -0.11 & -0.09 & -0.62 & -0.10 & -0.05 & -0.14 & -1.71 & 9.54 \\
\hline 0.336 & 17010 & 7.2109 & 090 & -0.72 & -0.09 & -0.08 & -0.58 & -0.09 & -0.04 & -0.13 & -1.52 & 9.69 \\
\hline 0.336 & 15840 & 7.2270 & & -0.67 & -0.06 & -0.08 & -0.53 & -0.08 & -0.03 & -0.13 & -1.33 & 9.85 \\
\hline 0.336 & 14640 & 7.2464 & & -0.61 & -0.03 & -0.07 & -0.47 & -0.07 & -0.02 & -0.12 & -1.12 & 10.03 \\
\hline 0.336 & 13540 & 7.2658 & 0.210 & -0.55 & 0.01 & -0.06 & -0.41 & -0.06 & -0.01 & -0.12 & -0.92 & 10.22 \\
\hline 0.336 & 12620 & 7.2830 & & -0.50 & 0.05 & -0.05 & -0.34 & -0.04 & 0.01 & -0.12 & -0.75 & 10.40 \\
\hline 0.336 & 11810 & 7.2986 & & -0.48 & 0.08 & -0.03 & -0.27 & -0.02 & 0.01 & -0.12 & -0.59 & 10.56 \\
\hline 0.336 & 10880 & 7.3163 & & -0.48 & 0.12 & 0.00 & -0.16 & 0.01 & 0.03 & -0.11 & -0.41 & 10.78 \\
\hline 0.336 & 10020 & 7.3328 & & -0.48 & 0.16 & 0.05 & 0.00 & 0.06 & 0.05 & -0.10 & -0.29 & 11.06 \\
\hline 0.336 & 9370 & 7.3454 & & -0.50 & 0.20 & 0.09 & 0.16 & 0.09 & 0.07 & -0.09 & -0.23 & 11.33 \\
\hline 0.336 & 8720 & 7.3573 & & -0.52 & 0.23 & 0.13 & 0.33 & 0.13 & 0.10 & -0.08 & -0.18 & 11.62 \\
\hline 0.336 & 8120 & 7.3681 & & -0.52 & 0.27 & 0.17 & 0.51 & 0.17 & 0.13 & -0.06 & -0.15 & 11.93 \\
\hline 0.336 & 7460 & 7.3795 & & -0.51 & 0.33 & 0.22 & 0.73 & 0.22 & 0.17 & -0.04 & -0.12 & 12.29 \\
\hline 0.336 & 6950 & 7.3883 & 1.640 & -0.47 & 0.39 & 0.26 & 0.93 & 0.26 & 0.20 & -0.03 & -0.11 & 12.61 \\
\hline 0.336 & 6390 & 7.3987 & 2.023 & -0.40 & 0.47 & 0.31 & 1.17 & 0.31 & 0.24 & 0.00 & -0.11 & 13.00 \\
\hline
\end{tabular}


Continúa tabla página anterior

\begin{tabular}{|c|c|c|c|c|c|c|c|c|c|c|c|c|}
\hline$M_{*} / \mathrm{M}_{\odot}$ & $\mathrm{T}_{\text {eff }}$ & $\log (g)$ & $E d a d(\mathrm{Gyr})$ & U-B & B-V & V-R & V-K & R-I & $\mathrm{J}-\mathrm{H}$ & H-K & $\mathrm{BC}$ & $\mathrm{M}_{V}$ \\
\hline 0.336 & 5870 & 7.4099 & 2.492 & -0.29 & 0.56 & 0.37 & 1.43 & 0.37 & 0.28 & 0.03 & -0.13 & 13.41 \\
\hline 0.336 & 5000 & 7.4416 & 3.926 & -0.05 & 0.77 & 0.50 & 1.96 & 0.50 & 0.34 & 0.08 & -0.26 & 14.32 \\
\hline 0.336 & 4610 & 7.4569 & 5.361 & 0.07 & 0.88 & 0.57 & 2.18 & 0.57 & 0.35 & 0.06 & -0.38 & 14.83 \\
\hline 0.336 & 4240 & 7.4669 & 6.985 & 0.18 & 0.97 & 0.63 & 2.16 & 0.63 & 0.20 & -0.01 & -0.44 & 15.29 \\
\hline 0.336 & 3890 & 7.4727 & 8.597 & 0.26 & 1.05 & 0.68 & 1.80 & 0.67 & -0.08 & -0.10 & -0.42 & 15.65 \\
\hline 0.336 & 3610 & 7.4760 & 9.997 & 0.32 & 1.10 & 0.70 & 1.28 & 0.68 & -0.26 & -0.25 & -0.34 & 15.90 \\
\hline 0.336 & 3300 & 7.4790 & 11.832 & 0.40 & 1.16 & 0.72 & 0.76 & 0.64 & -0.36 & -0.34 & -0.21 & 16.17 \\
\hline 0.336 & 3020 & 7.4813 & 14.033 & 0.47 & 1.21 & 0.70 & 0.22 & 0.51 & -0.39 & -0.43 & -0.05 & 16.39 \\
\hline 0.449 & 36370 & 7.2604 & 0.002 & -1.16 & -0.28 & -0.14 & -1.00 & -0.18 & -0.12 & -0.17 & -3.45 & 8.13 \\
\hline 0.449 & 34060 & 7.2999 & 0.004 & -1.13 & -0.27 & -0.14 & -0.99 & -0.18 & -0.11 & -0.17 & -3.27 & 8.34 \\
\hline 0.449 & 32270 & 7.3577 & 0.011 & -1.11 & -0.26 & -0.14 & -0.97 & -0.17 & -0.11 & -0.17 & -3.13 & 8.58 \\
\hline 0.449 & 30310 & 7.4037 & 0.023 & -1.09 & -0.25 & -0.13 & -0.94 & -0.17 & -0.10 & -0.17 & -2.98 & 8.82 \\
\hline 0.449 & 28220 & 7.4342 & 035 & -1.05 & -0.23 & -0.13 & -0.90 & -0.16 & -0.10 & -0.17 & -2.82 & 9.04 \\
\hline 0.449 & 26220 & 7.4605 & 0.049 & -1.01 & -0.21 & -0.12 & -0.86 & -0.15 & -0.09 & -0.16 & -2.64 & 9.24 \\
\hline 0.449 & 24310 & 7.4844 & 0.068 & -0.97 & -0.19 & -0.12 & -0.81 & -0.14 & -0.08 & -0.15 & -2.45 & 9.44 \\
\hline 0.449 & 22530 & 7.5065 & 093 & -0.93 & -0.16 & -0.11 & -0.76 & -0.13 & -0.07 & -0.15 & -2.25 & 9.63 \\
\hline 0.449 & 20860 & 7.5270 & 0.128 & -0.88 & -0.14 & -0.10 & -0.71 & -0.12 & -0.06 & -0.14 & -2.05 & 9.81 \\
\hline 0.449 & 19290 & 7.5462 & 0.174 & -0.83 & -0.11 & -0.10 & -0.66 & -0.11 & -0.05 & -0.14 & -1.84 & 9.99 \\
\hline 0.449 & 17830 & 7.5638 & 0.237 & -0.78 & -0.09 & -0.09 & -0.61 & -0.10 & -0.04 & -0.14 & -1.63 & 10.17 \\
\hline 0.449 & 16470 & 7.5796 & 0.320 & -0.72 & -0.06 & -0.08 & -0.56 & -0.09 & -0.03 & -0.13 & -1.42 & 10.34 \\
\hline 0.449 & 15200 & 7.5938 & 0.429 & -0.66 & -0.02 & -0.07 & -0.50 & -0.07 & -0.02 & -0.13 & -1.21 & 10.51 \\
\hline 0.449 & 14030 & 7.6063 & 0.570 & -0.60 & 0.01 & -0.07 & -0.44 & -0.06 & -0.01 & -0.12 & -1.00 & 10.69 \\
\hline 0.449 & 12920 & 7.6173 & 0.749 & -0.55 & 0.06 & -0.05 & -0.36 & -0.04 & 0.00 & -0.12 & -0.80 & 10.87 \\
\hline 0.449 & 11890 & 7.6273 & 0.982 & -0.54 & 0.09 & -0.03 & -0.28 & -0.02 & 0.01 & -0.12 & -0.59 & 11.04 \\
\hline 0.449 & 10910 & 7.6365 & 1.289 & -0.53 & 0.13 & 0.01 & -0.15 & 0.02 & 0.03 & -0.11 & -0.41 & 11.26 \\
\hline 0.449 & 10030 & 7.6452 & 1.672 & -0.53 & 0.17 & 0.05 & 0.02 & 0.06 & 0.05 & -0.10 & -0.30 & 11.54 \\
\hline 0.449 & 9220 & 7.6532 & & -0.54 & 0.21 & 0.10 & 0.21 & 0.11 & 0.08 & -0.09 & -0.23 & 11.85 \\
\hline 0.449 & 8460 & 7.6606 & 2.728 & -0.55 & 0.25 & 0.15 & 0.41 & 0.15 & 0.11 & -0.07 & -0.18 & 12.19 \\
\hline 0.449 & 7770 & 7.6674 & 3.349 & -0.54 & 0.30 & 0.20 & 0.63 & 0.20 & 0.15 & -0.05 & -0.14 & 12.54 \\
\hline 0.449 & 7130 & 7.6741 & 3.969 & -0.49 & 0.36 & 0.24 & 0.86 & 0.25 & 0.19 & -0.03 & -0.12 & 12.91 \\
\hline 0.449 & 6540 & 7.6806 & 4.611 & -0.42 & 0.44 & 0.30 & 1.10 & 0.30 & 0.23 & -0.01 & -0.10 & 13.28 \\
\hline 0.449 & 6020 & 7.6874 & 5.330 & -0.32 & 0.54 & 0.35 & 1.34 & 0.35 & 0.26 & 0.02 & -0.12 & 13.67 \\
\hline 0.449 & 5540 & 7.6959 & 6.189 & -0.21 & 0.63 & 0.41 & 1.59 & 0.41 & 0.29 & 0.05 & -0.14 & 14.07 \\
\hline 0.449 & 5110 & 7.7067 & 7.472 & -0.09 & 0.74 & 0.48 & 1.88 & 0.48 & 0.33 & 0.07 & -0.24 & 14.55 \\
\hline 0.449 & 4710 & 7.7160 & 9.455 & 0.04 & 0.85 & 0.55 & 2.10 & 0.55 & 0.34 & 0.05 & -0.34 & 15.04 \\
\hline 0.449 & 4330 & 7.7221 & 11.582 & 0.14 & 0.94 & 0.61 & 2.10 & 0.61 & 0.20 & -0.01 & -0.41 & 15.49 \\
\hline 0.449 & 3970 & 7.7258 & 13.651 & 0.22 & 1.02 & 0.66 & 1.79 & 0.65 & -0.06 & -0.09 & -0.40 & 15.85 \\
\hline 0.449 & 3640 & 7.7282 & 15.838 & 0.30 & 1.08 & 0.69 & 1.19 & 0.66 & -0.28 & -0.26 & -0.31 & 16.16 \\
\hline
\end{tabular}

Las edades se cuentan desde el fin de la transferencia de masa.

\subsection{Conclusiones}

En este estudio se ha explorado la evolución de enanas blancas con núcleos de helio (HeWDs) con progenitores de metalicidades mucho menores que la solar. Los modelos presentados aquí son 
apropiados para la interpretación de recientes y futuras observaciones de HeWDs en cúmulos globulares. Específicamente, se han considerado dos valores de metalicidad: $Z=0.001$ y $Z=0.0002$. En el caso de $Z=0.0002$, las masas estelares consideradas fueron $0.199,0.209$, $0.219,0.225,0.243,0.266,0.280,0.300,0.319 \mathrm{M}_{\odot}$ y en el caso de $Z=0.001$ fueron 0.172 , $0.183,0.197,0.230,0.244,0.300,0.336,0.380,0.390,0.422,0.449 \mathrm{M}_{\odot}$. Todos estos modelos fueron evolucionados desde el final de la etapa de pérdida de masa hasta etapas muy avanzadas de su enfriamiento. La naturaleza binaria de nuestros modelos de HeWD ha sido simulada quitándole masa a un modelo de $1 \mathrm{M}_{\odot}$ en momentos apropiados durante su evolución posterior a la etapa de secuencia principal. Específicamente, para obtener modelos iniciales de pre-HeWD apropiados, las tasas de transferencia de masa fueron obtenidas imponiendo que el radio estelar debe permanecer cercano al radio del lóbulo de Roche. Un tratamiento completamente noconservativo ha sido adoptado en todos los cálculos y se ha considerado la pérdida de momento angular del sistema por pérdida de masa, radiación de ondas gravitatorias y frenado magnético.

La evolución de nuestros modelos de HeWD ha sido calculada autoconsistentemente con las predicciones de la difusión de elementos y la combustión nuclear. Un tratamiento no-gris de la atmósfera ha sido también incluido. Los modelos de atmósfera están basados en un tratamiento detallado de la microfísica incorporada en el mismo, tal como efectos no-ideales en la ecuación de estado (Hummer \& Mihalas, 1988) y la inclusión de las opacidades por líneas (series de Balmer, Lyman y Paschen) y pseudo-continuo. También, una actualización de los datos sobre las opacidades CIA ha sido incorporada en los presentes cálculos. Tal descripción nos permite obtener índices de color y magnitudes en etapas tanto tempranas como tardías de la evolución.

Una característica importante predicha por los presentes cálculos es la existencia de episodios de flash termonucleares en la mayor parte de las secuencias de HeWDs. En parte, este es el resultado de incluir en nuestros cálculos los diferentes procesos de difusión microscópica. Encontramos que cuanto más baja es la metalicidad, más grande es la masa mínima para la ocurrencia de los flashes CNO. Específicamente, para $Z=0.001$ y $Z=0.0002$ esta masa límite es $M \approx 0.22$ y $0.26 \mathrm{M}_{\odot}$ respectivamente, mientras que para progenitores de metalicidad solar este valor es de $\approx 0.18 \mathrm{M}_{\odot}$ (ver capítulo 3). Además, los flashes CNO son menos intensos a medida que el contenido de metales de la estrella es menor y el rango de masas para el cual los flashes ocurren dependen de la inclusión de la difusión en los cálculos evolutivos. También encontramos que la existencia de esta masa límite para la ocurrencia de los flashes CNO inducidos por la difusión produce una dicotomía en las edades de los modelos de HeWD, particularmente notoria para $Z=0.001$ entre los modelos que sufren y los que no sufren estos flashes. Aquellos modelos que no sufren flashes CNO evolucionan lentamente a lo largo de la rama de enfriamiento por efectos de la combustión nuclear residual del hidrógeno, mientras que en el caso de los modelos que sufren flashes CNO, su evolución en la rama de enfriamiento es rápida debido a que las reacciones nucleares juegan un papel casi despreciable como fuente de energía de la estrella, alcanzando $\mathrm{T}_{\text {eff }}$ muy bajas en menos de 15 Gyr.

Otro resultado que encontramos en nuestros cálculos es que algunos de nuestros modelos de HeWD experimentan varios episodios de flashes de hidrógeno vía las cadenas protón-protón (PP). Estos flashes de PP, que tienen lugar en etapas más avanzadas de la evolución que los de CNO, son experimentados por todas nuestras secuencias con $Z=0.0002$. Además, nuestras secuencias de 0.172 y $0.183 \mathrm{M}_{\odot}$ con $Z=0.001$ también los experimentan, pero a edades muy grandes. Encontramos que estas inestabilidades PP son inducidas por la difusión de elementos que transporta hidrógeno hacia el interior de la estrella donde se enciende de forma inestable. Excepto para los modelos menos masivos $\left(M \leq 0.25 \mathrm{M}_{\odot}\right)$, los flashes de PP tienen lugar entre 2 y 4 Gyr después del final de los episodios de pérdida de masa, y en general cuanto más masiva 
la HeWD, antes en su vida estos flashes ocurren.

La evolución de nuestros modelos de HeWD ha sido también analizada en diagramas colormagnitud. Encontramos que los modelos que han sufrido flashes CNO exhiben un turn-off in la mayoría de sus índices de color a $M_{V} \approx 16$. Este turn-off, que resulta de la fuerte absorción por las opacidades CIA del hidrógeno molecular a bajas temperaturas, es alcanzado bien dentro de los 15 Gyr de vida de la estrella, principalmente por los modelos con $Z=0.001$. Finalmente, las predicciones de nuestros modelos en los diagramas color-magnitud han sido comparadas con recientes datos observacionales de candidatas a HeWDs en los cúmulos globulares NGC 6397 y 47 Tucanae (Taylor et al., 2001; Edmonds et al., 2001, respectivamente). En relación a esto, encontramos que las tres candidatas más brillantes a HeWDs en NGC 6397 pueden identificarse como HeWDs caracterizadas por masas entre 0.20 y $0.22 \mathrm{M}_{\odot}$ (por debajo de la masa límite para la ocurrencia de flashes CNO) y edades entre 0.5 y 1.5 Gyr. sin embargo, en el caso de las tres candidatas menos brillantes de este cúmulo, el acuerdo observacional no es tan evidente como en el caso de los objetos más brillantes. En efecto, nuestros modelos parecen ser un poco más masivos que lo requerido por las observaciones. De hecho, nuestro tratamiento de pérdida de masa da origen a un límite mínimo de $\sim 0.2 \mathrm{M}_{\odot}$ para una HeWD remanente de un progenitor de $Z=0.0002$. Sin embargo, vale la pena mencionar que un valor $\Delta(V-I) \approx 0.1$ en la incerteza total en este índice de color permitiría un buen acuerdo entre las predicciones teóricas y las observaciones. En este sentido, la incerteza en las observaciones en $(V-I)$ para la candidata a HeWD en 47 Tucanae está muy cerca a este valor (ver figura $5.10^{4}$; las observaciones en ambos cúmulos han sido realizadas con los mismos instrumentos, la WFPC2 del Hubble Space Telescope). Finalmente, la HeWD en 47 Tucanae es particularmente relevante porque la edad de spin-down del púlsar de milisegundo compañero de la HeWD permite obtener una estimación independiente de la edad de la HeWD. Específicamente, la edad del púlsar de 2 Gyr (Edmonds et al., 2001) está en acuerdo con las predicciones de nuestras secuencias de $Z=0.001$. Además, los datos observacionales para $M_{V}$ y $U-V$ son consistentes con nuestra secuencia de $0.17 \mathrm{M}_{\odot}$.

\footnotetext{
${ }^{4}$ Hay que mencionar que Townsley \& Bildsten (2002) han sugerido que las tres candidatas menos brillantes en NGC 6397 podrían ser variables cataclísmicas involucrando una WD de carbono-oxígeno y una estrella de secuencia principal de baja masa. Sin embargo la evidencia observacional parece no soportar esta hipótesis (Edmonds 2002; comunicación privada).
} 


\section{Capítulo 6}

\section{Enanas blancas de carbono-oxígeno. Progenitor de $3 \mathbf{M}_{\odot}$}

Es probable que la estructura interna de la enanas blancas con núcleos de carbono-oxígeno (COWD) resulte el tipo de estructura estelar menos complicada de modelar, al menos durante la mayor parte de la vida de estas estrellas (la cristalización del interior de estas estrellas a bajas temperaturas efectivas es claramente una excepción a esta afirmación y resulta aún hoy un tema de estudio completamente abierto). Sin embargo, el gran desarrollo de la astrosismología en los últimos años ha proporcionando una herramienta de fundamental importancia para develar los detalles de la estructura interna de las COWDs, demostrando que los modelos de estructura de las COWDs desarrollados en el pasado son generalmente sobre-simplificados. La necesidad de obtener modelos de COWDs lo más detallados posible nos lleva a estudiar la estructura de estos objetos comenzando por la evolución de sus progenitores, partiendo desde la ZAMS y siguiendo su evolución hasta la formación de la COWD. En este capítulo se presentan los resultados correspondientes a una estrella con una masa inicial igual a $3 \mathrm{M}_{\odot}$, que da origen a una COWD de $0.56 \mathrm{M}_{\odot}$, masa representativa de la mayoría de las COWD.

Los resultados presentados en este capítulo pueden encontrarse en Althaus, Serenelli, Córsico \& Benvenuto (2002).

\subsection{Introducción}

Durante los últimos años, las pulsaciones estelares radiales y particularmente las no-radiales se han convertido en una herramienta poderosa para investigar la estructura interna y la evolución de las estrellas. Gracias al aumento en el grado de sofisticación en las técnicas tanto observacionales como teóricas, la astrosismología ha sido aplicada exitosamente al análisis de los patrones de oscilaciones de numerosas estrellas pulsantes, dentro de las cuales el Sol representa el mejor ejemplo. Con el avance y refinamiento de las observaciones, un gran número de objetos estelares ubicados en diferentes lugares en el HRD han mostrado presentar pulsaciones no-radiales. En efecto, estrellas variables correspondientes de diferentes estadios evolutivos como roAp, SPB, $\delta$ Scuti, $\beta$ Cephei y enanas blancas variables han sido clasificadas como pulsadores no-radiales (ver, por ejemplo Cox 1980; Unno et al. 1989; Brown \& Gilliland 1994; Gautschy \& Saio 1995, 1996).

Desde el punto de vista observacional (con la obvia excepción del Sol), las enanas blancas representan una de las mejor establecidas y estudiadas clases de pulsadores no-radiales. Las 
enanas blancas pulsantes exhiben variaciones de luminosidad multiperiódicas en tres regiones diferentes del HRD correspondientes a las actualmente llamadas DOV (y PNNV), DBV y DAV (ver, por ejemplo, Winget 1988). De interés particular en el trabajo presentado en esta tesis son las DAVs (COWDs con atmósferas ricas en hidrógeno), también conocidas como estrellas ZZ Ceti, que presentan pulsaciones en la banda de inestabilidad correspondiente al rango de $12500 \geq \mathrm{T}_{\text {eff }} \geq 10700 \mathrm{~K}$. Las periodicidades en sus curvas de luz son explicadas básicamente en términos de modos-g no-radiales de grado armónico bajo $(\ell \leq 2)$, producidos por el mecanismo $\kappa-\gamma$ operando en una región de ionización parcial debajo de la superficie estelar (Dolez \& Vauclair, 1981; Winget et al., 1982) ${ }^{1}$. Los períodos observados en estas estrellas están en el rango 100-1200s y las amplitudes fotométricas alcanzan hasta 0.30mag. Numerosos estudios han sido dedicados al análisis de las características pulsacionales de las DAVs. Entre ellos mencionamos los trabajos de Tassoul, Fontaine \& Winget (1990); Brassard et al. (1991, 1992a,b); Bradley \& Winget (1994); Gautschy, Ludwig \& Freytag (1996); Bradley (1996, 1998, 2001).

Para comprender completamente las propiedades de las oscilaciones de las DAVs y obtener de esta manera la mayor ventaja de la riqueza ofrecida por las observaciones disponibles, se requieren modelos estelares que incluyan una física detallada. En el contexto de estas estrellas pulsantes, la mayor parte de las investigaciones existentes descansan en modelos estelares construidos bajo la suposición de algunas hipótesis simplificatorias. Esto es cierto en particular en cuanto al tratamiento de la distribución de las abundancias químicas en el interior estelar. En este sentido, los perfiles de carbono y oxígeno son usualmente tratados como parámetros libres. Además, la distribución de las abundancias químicas se asume fija durante la evolución a lo largo del dominio de inestabilidad y en algunos casos la aproximación de equilibrio difusivo es empleada para determinar la forma del perfil químico en las regiones de interface. La combustión del hidrógeno es también despreciada en la construcción de los modelos evolutivos empleados para los estudios pulsacionales de las estrellas ZZ Ceti. Esto está justificado solamente si la enana blanca es formada con capas de hidrógeno relativamente delgadas. Sin embargo, si la envoltura de hidrógeno es lo suficientemente masiva, las reacciones nucleares de combustión del hidrógeno consituyen una fuente apreciable de energía aún durante las etapas evolutivas correspondientes al dominio de las estrellas ZZ Ceti (Iben \& Tutukov, 1984). Los detalles de la combustión son más complejos cuando la difusión de los elementos es tenida en cuenta. En efecto, los cálculos evolutivos de enanas blancas en los cuales la dependencia temporal de la difusión de elementos es incorporada apropiadamente (Iben \& MacDonald, 1986) muestran que la combustión nuclear del hidrógeno a través de las reacciones del ciclo CN juega un rol diferente dependiendo de la masa de la región del buffer de helio entre la envoltura rica en hidrógeno y las capas más internas ricas en helio. Por ejemplo, un flash de hidrógeno inducido por la difusión es esperado si el buffer de helio es suficientemente poco masivo. Cuán masivo resulta el buffer de helio puede depender críticamente de la fase en el ciclo de flashes del shell de helio durante la etapa de pulsos térmicos en la cual la estrella progenitora se aparta de la rama asintótica de las gigantes (AGB) (para más detalles ver D'Antona \& Mazzitelli 1990).

La construcción de modelos estelares de enanas blancas DAV apropiadas para estudios pulsacionales en los que las cuestiones mencionadas anteriormente sean incorporadas consistentemente requiere cálculos evolutivos que consideren no sólo la dependencia temporal de la difusión de los elementos químicos sino también un tratamiento detallado de las etapas evolutivas previas a la formación de la enana blanca. El cálculo de dichos modelos estelares es

\footnotetext{
${ }^{1}$ Sin embargo, Brickhill (1991) propone un mecanismo producido por la convección como responsable de la sobre-estabilidad de los modos-g en DAVs (ver también Goldreich \& Wu 1999).
} 
el principal objetivo del trabajo presentado en este capítulo. La aplicación principal de los modelos de enana blanca DA presentados aquí será la exploración de sus propiedades pulsacionales, sin embargo tal estudio queda más allá del alcance del presente trabajo, limitándonos a la discusión de los resultados evolutivos y a sus implicaciones para las cantidades principales involucradas en las ecuaciones de pulsaciones adiabáticas.

La evolución de la enana blanca tratada de manera autoconsistente con el formalismo dependiente del tiempo para la difusión de elementos es un aspecto fundamental en el presente trabajo. En la mayor parte de los estudios previos sobre pulsaciones, el equilibrio difusivo en la aproximación de elemento-traza ha sido usada para establecer la forma del perfil químico en la región de transición de composición de los modelos evolutivos (ver Tassoul, Fontaine \& Winget 1990; Brassard et al. 1991, 1992a,b; Bradley 1996, 1998, 2001; Bradley \& Winget 1994; Montgomery, Metcalfe \& Winget 2001). Sin embargo, la aproximación de equilibrio para la difusión no es válida cuando las escalas temporales de la difusión son comparables a las evolutivas. En particular, el equilibrio difusivo en las capas profundas del modelo de enana blanca no es una aproximación adecuada aún a las edades características de la etapa de ZZ Ceti. En efecto, durante tales etapas se encuentra que la difusión modifica la distribución espacial de los elementos, particularmente en las interfaces químicas (ver Iben \& MacDonald 1985). Para un tratamiento correcto de las estratificaciones químicas que evolucionan por efectos de la difusión se consideran en estos modelos los procesos de sedimentación gravitatoria y difusión química y térmica siguiendo el tratamiento de Burgers (1969) para gases multicomponentes. La forma de la zona de transición de la composición es muy importante desde el punto de vista de la astrosismología. En particular, contribuye a la forma del término de Ledoux que aparece en la frecuencia de Brunt-Väisälä (Brassard et al., 1991) y juega un rol crítico en el fenómeno de los "modos atrapados" en enanas blancas (ver Tassoul, Fontaine \& Winget 1990; Brassard et al. 1992a y referencias ahí citadas).

Otro aspecto importante del presente estudio es que las etapas evolutivas previas a la formación de la enana blanca son calculadas consistentemente. Específicamente, comenzamos nuestros cálculos de un modelo de $3 \mathrm{M}_{\odot}$ en la ZAMS y seguimos su evolución durante las etapas de combustión de hidrógeno y helio en el núcleo hasta el extremo de la AGB donde tiene lugar la etapa de pulsos térmicos en el shell de helio. Para obtener perfiles químicos cuya estructura en la región del helio esté relajada (Mazzitelli \& D'Antona, 1986), computamos un número razonable de pulsos térmicos después de los cuales el progenitor es forzado a evolucionar hacia su configuración de enana blanca invocando un fuerte episodio de pérdida de masa. Nos concentraremos en la situación particular en la cual el progenitor de enana blanca se aparta de la AGB cuando la combustión de helio estacionaria es el principal soporte de la luminosidad de la estrella, posterior a la ocurrencia del último pulso térmico. No se ha invocado pérdida de masa adicional durante la etapa de nebulosa planetaria o durante las etapas tempranas de evolución a lo largo de la rama de enfriamiento. Esto permite examinar la valor máximo de la masa para el contenido remanente de hidrógeno predicho en el caso particular de la evolución que se presenta en este trabajo. De esta manera podemos estudiar el rol que desempeña la combustión nuclear durante las etapas de enfriamiento.

\subsection{Detalles computacionales}

Los cálculos han sido realizados empleando el CEELP descripto en el capítulo 2. Algunos detalles, sin embargo, merecen un comentario específico, en particular en relación al tratamiento 
del borde del núcleo convectivo de helio y el valor adoptado para el rate de la reacción nuclear ${ }^{12} \mathrm{C}(\alpha, \gamma){ }^{16} \mathrm{O}$. Es bien sabido que la determinación experimental de este rate presenta grandes incertezas. Por otro lado esta reacción resulta crucial en la determinación de la composición química final del núcleo. Ha habido diversos intentos de acotar el rate ${ }^{12} \mathrm{C}(\alpha, \gamma){ }^{16} \mathrm{O}$ en base a datos astrofísicos. Sin embargo, las fracciones de ${ }^{12} \mathrm{C}$ y ${ }^{16} \mathrm{O}$ producidas en un interior estelar típico dependen no solamente del rate de la reacción sino también del tratamiento dado a la convección. De esta manera, en base a datos astrofísicos no es posible separar ambos efectos y éstos sólo permiten obtener una sección eficaz "efectiva" para la reacción ${ }^{12} \mathrm{C}(\alpha, \gamma){ }^{16} \mathrm{O}$, mientras no se cuente con una teoría confiable para la convección. En este trabajo adoptamos para esta reacción el rate dado por Angulo C. et al. (1999), que es aproximadamente un factor 2 más grande que el dado por Caughlan \& Fowler (1988). Thielemann, Nomoto \& Hashimoto (1996) han realizado un estudio del colapso gravitacional de una supernova y comparado las cantidades predichas de ${ }^{12} \mathrm{C}$ y ${ }^{16} \mathrm{O}$ con las abundancias observadas en SN 1987A y SN 1993J. Ellos encuentran un acuerdo excelente cuando el rate de la reacción ${ }^{12} \mathrm{C}(\alpha, \gamma){ }^{16} \mathrm{O}$ es el de Caughlan et al. (1985) y el criterio de Schwarzschild sin overshooting es utilizado para el tratamiento de la convección. Dado que los valores dados para el rate de la reacción ${ }^{12} \mathrm{C}(\alpha, \gamma){ }^{16} \mathrm{O}$ por Caughlan et al. (1985) y Angulo C. et al. (1999) son muy parecidos en todo el rango de temperaturas de interés adoptamos, para determinar los bordes convectivos, el criterio de Schwarzschild sin overshooting a lo largo de este trabajo.

En este trabajo seguimos la evolución de una estrella de, inicialmente, $3 \mathrm{M}_{\odot}$ comenzando desde la ZAMS (masa representativa de una estrella de masa intermedia, progenitor de COWD). Dado que las estrellas DA alcanzan la banda de inestabilidad cuando su edad de enfriamiento es del orden de algunos centenares de millones de años (el valor preciso depende por supuesto de la masa de la COWD) es razonable suponer que un progenitor de $3 \mathrm{M}_{\odot}$ tenga una composición química inicial típica de una estrella de población I (que en este caso asumimos como solar, ver Anders \& Grevesse 1989), por lo tanto la metalicidad $Z$ adoptada es $Z=0.02$ y las abundancias iniciales de hidrógeno y helio son $X=0.705$ y $Y=0.275$ respectivamente. La evolución ha sido seguida a masa estelar constante durante las etapas de combustión de hidrógeno y helio en el núcleo estelar hasta el extremo de la AGB, donde ocurren los pulsos térmicos de helio. Para obtener la configuración de enana blanca se ha incorporado en el código de evolución una pérdida de masa artificial. En particular, la pérdida de masa fue considerada después de que el modelo experimentó el 11avo pulso térmico. El valor de la pérdida de masa adoptada es de $10^{-4} \mathrm{M}_{\odot} \mathrm{yr}^{-1}$ y fue aplicada a cada modelo estelar a medida que la evolución continuaba. Después de la convergencia de cada nuevo modelo estelar, la masa estelar total es reducida de acuerdo al paso temporal utilizado para el modelo.

Un aspecto importante de este estudio es el tratamiento de la evolución de la distribución de las abundancias químicas causada por los procesos de difusión durante la etapa de enana blanca. Como ya se mencionó, estos procesos incluyen la sedimentación gravitatoria, la difusión química y la térmica y el tratamiento adoptado es para gases multicomponentes presentado por Burgers (1969), evitando de esta manera el uso de la aproximación de elemento-traza, usualmente empleado en los estudios de enanas blancas. Es importante destacar que cuando el progenitor de enana blanca se aparta de la AGB después de los episodios de pérdida de masa, su envoltura está formada por una mezcla de hidrógeno y helio. También en su interior, debajo de una delgada capa casi pura de helio (buffer de helio), hay una región rica en helio y carbono (ver próxima sección); por lo tanto la aproximación de elemento-traza resulta claramente inválida en estas condiciones. En este estudio estamos interesados en la evolución química que ocurre dentro de la estrella, por lo tanto procesos tales como la levitación radiativa y pérdida de masa 
durante las etapas de $\mathrm{T}_{\text {eff }}$ altas al principio de la rama de enfriamiento, que pueden alterar la composición superficial de la estrella (ver Unglaub \& Bues 2000 para un estudio detallado de la evolución química superficial de enanas blancas calientes), no han sido considerados. En el contexto de las enanas blancas DA el tratamiento que ha sido empleado ha sido utilizado por Iben \& MacDonald $(1985,1986)$ (sin embargo en esos trabajos no se incluye la difusión térmica). Los detalles concernientes al procedimiento para la solución de las ecuaciones de difusión pueden encontrarse en la sección 3.2. Los elementos considerados en las ecuaciones de difusión son ${ }^{1} \mathrm{H},{ }^{3} \mathrm{He},{ }^{4} \mathrm{He},{ }^{12} \mathrm{C},{ }^{14} \mathrm{~N}$ y ${ }^{16} \mathrm{O}$. Finalmente, es importante mencionar que las opacidades radiativas durante la etapa de enana blanca han sido calculadas para metalicidades consistentes con las predicciones de la difusión. En particular, en cada punto del modelo estelar, la metalicidad ha sido tomada igual a dos veces la abundancia de los elementos CNO, como sugiere Iben \& Tutukov (1986).

\subsection{Resultados evolutivos}

\subsubsection{Progenitor de enana blanca}

En esta sección se describen los resultados obtenidos en relación a la evolución de las etapas previas a la formación de la enana blanca. Estas etapas, que incluyen la evolución desde la secuencia principal hasta la AGB, han sido estudiadas numerosas veces por otros investigadores por lo que la descripción que se presenta aquí se limita aquellas características que son de relevancia inmediata para la formación de la enana blanca. Referimos al lector interesado a los trabajos de Mazzitelli \& D'Antona (1986); Vassiliadis \& Wood (1993); Blöcker (1995a) para una descripción más detallada de la evolución de estrellas de masas bajas e intermedias. Comenzamos examinando el track evolutivo completo en el HRD que se muestra en la figura 6.1. La simulación numérica para una estrella de, inicialmente, $3 \mathrm{M}_{\odot}$ cubre todas las etapas evolutivas desde la ZAMS hasta el dominio de las estrellas ZZ Ceti en la rama de enfriamiento de las enanas blancas. La edad (en unidades de $10^{4}$ años) desde el fin de la pérdida de masa, y la masa de hidrógeno (en unidades de $10^{-4} \mathrm{M}_{\odot}$ ) están indicadas en algunos puntos seleccionados a lo largo del track. Las fases evolutivas correspondientes a la pérdida de masa no se muestran por claridad.

Después de $4.1 \times 10^{8}$ años de evolución y hacia el final de la combustión de helio en el núcleo la primer característica digna de ser comentada es la aparición de una serie de micropulsos (que no deben ser confundidos con los pulsos térmicos que ocurren en la AGB) de baja amplitud en la luminosidad superficial. Tales micropulsos son causados por inestabilidades térmicas en el shell que rodea al núcleo rico en carbono y oxígeno donde el helio se está quemando, las cuales causan que la luminosidad de helio sufra oscilaciones. Específicamente, estos micropulsos ocurren cuando la abundancia por masa central de helio cae por debajo de $\approx 0.001$ (y el núcleo convectivo desaparece). La dependencia temporal de la luminosidad superficial $L_{*}$ y de helio $L_{H e}$ (en unidades solares) durante la fase de los micropulsos se muestran en la figura pequeña incorporada en la figura 6.1, donde el tiempo está medido en Myr desde la ZAMS. Un total de 40 micropulsos con un período interpulso de $\approx 3.1 \times 10^{5}$ años ocurren en esta fase y la amplitud de $L_{*}$ es a lo sumo de $\Delta \log \left(L_{*} / \mathrm{L}_{\odot}\right)=0.02$. Cabe mencionar que este fenómeno ha sido también encontrado en un modelo estelar de $3 \mathrm{M}_{\odot}$ por Mazzitelli \& D'Antona (1986). Después del final de los micropulsos la estrella evoluciona hasta que el helio es completamente agotado en el centro. La duración total de la combustión de helio en el núcleo es de unos $1.25 \times 10^{8}$ años. 


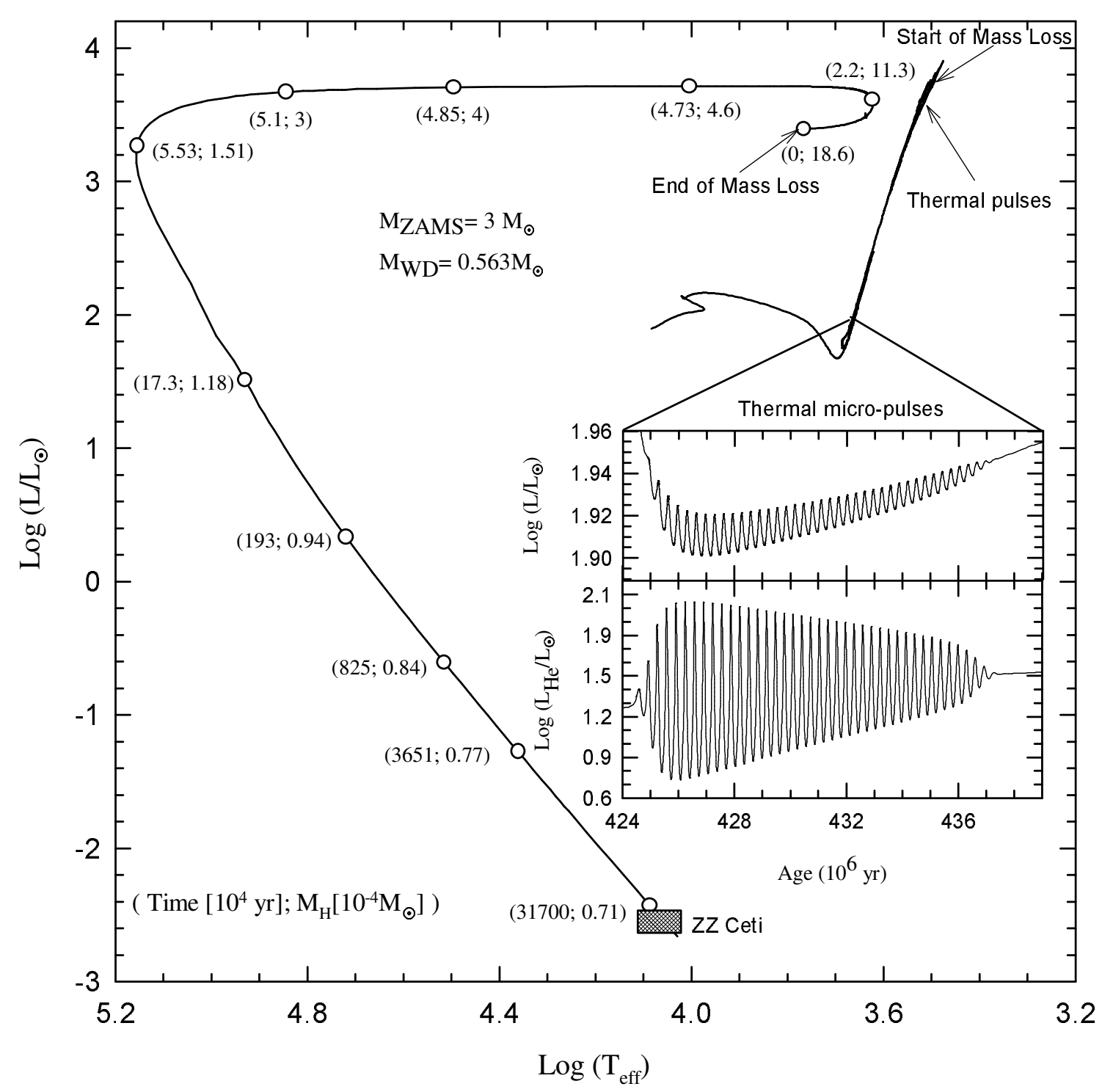

Figura 6.1: Diagrma Hertzsprung-Russell para la evolución del modelo de $3 \mathrm{M}_{\odot}$, desde la ZAMS hasta la etapa de enana blanca. Por claridad las etapas evolutivas correspondientes a la pérdida de masa no se muestran. Los número en paréntesis junto a los círculos a lo largo del track indican la edad (en $10^{4}$ años) medida desde el fin de la pérdida de masa y la masa de hidrógeno in las capas externas en unidades de $10^{4}$ años. El dominio de las estrellas ZZ Ceti, correspondiente a la banda de inestabilidad se muestra como una región sombreada. Como resultado de los episodios de pérdida de masa, la masa estelar decrece de $3 \mathrm{M}_{\odot}$ a $0.563 \mathrm{M}_{\odot}$. Notar que después del fin de la pérdida de masa, la estrella retorna hacia bajas temperaturas efectivas donde se quema una apreciable fracción de su contenido de hidrógeno. Durante esta fase la evolución ocurre lentamente. El gráfico pequeño muestra la evolución de las luminosidades superficial y de combustión del helio (en unidades solares) durante la fase de micropulsos térmicos, hacia el final de la combustión de helio en el núcleo de la estrella. 

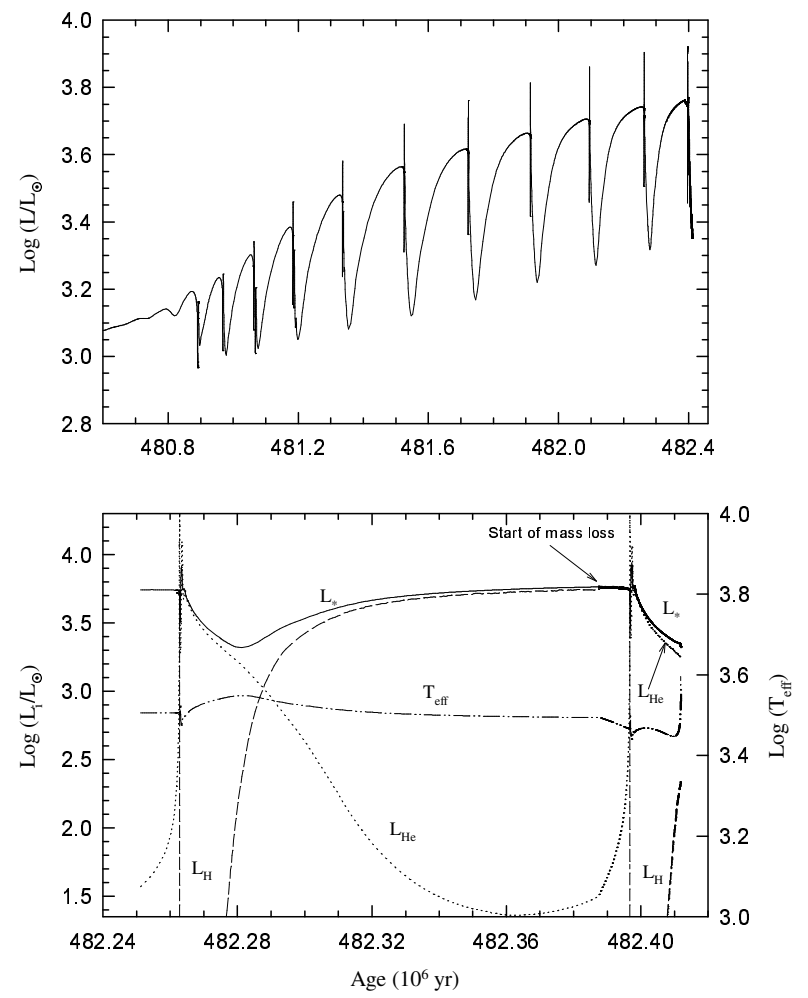

Figura 6.2: Panel superior: evolución de la luminosidad superficial (en unidades solares) durante la fase correspondiente a los pulsos térmicos de helio en el extremo de la AGB. El modelo experimenta 11 pulsos térmicos antes de apartarse de la AGB como resultado de la pérdida de masa. La escala de tiempo está dada en Myr contados desde la ZAMS. Panel inferior: evolución de la luminosidad superficial $L_{*}$, luminosidad por combustión del helio $L_{H e}$, luminosidad por combustión del hidrógeno $L_{H}$ y temperatura efectiva ( $\mathrm{T}_{\text {eff }}$ ) durante y entre los pulsos 10mo. y 11avo. Notar que el modelo se aparta de la AGB poco después del pico del 11avo. pulso, cuando la combustión del shell de helio es la fuente de energía dominante.

Después de que el helio es agotado en el centro, dejando una abundancia central de oxígeno de 0.62 por masa, la evolución procede hacia la fase de los pulsos térmicos en el extremo de la AGB, punto en el cual la combustión de helio en el shell se vuelve inestable nuevamente. Es bien sabido que la evolución en la AGB de las estrellas de masa intermedia está caracterizada por flashes en el shell de helio durante los cuales la liberación de energía nuclear aumenta rápidamente. En esta simulación el tiempo transcurrido desde que el helio se agota en el centro hasta el primer pulso térmico es $2.6 \times 10^{7}$ años, cuando la luminosidad superficial excede $\log \left(L / \mathrm{L}_{\odot}\right)=3.2$ por primera vez en su evolución. Después de experimentar 11 pulsos térmicos y considerable pérdida de masa, la masa de la envoltura de hidrógeno es reducida tanto que la estrella no puede mantener una envoltura convectiva extendida y se contrae, apartándose de la AGB, evolucionando hacia temperaturas efectivas altas. Esto tiene lugar cuando la luminosidad de la estrella es soportada por la combustión estacionaria del helio. Las implicaciones de este hecho serán discutidas en la próxima sección, por ahora basta decir que una vez que la pérdida de masa finaliza, el objeto remanente consume una considerable fracción de su hidrógeno remanente 


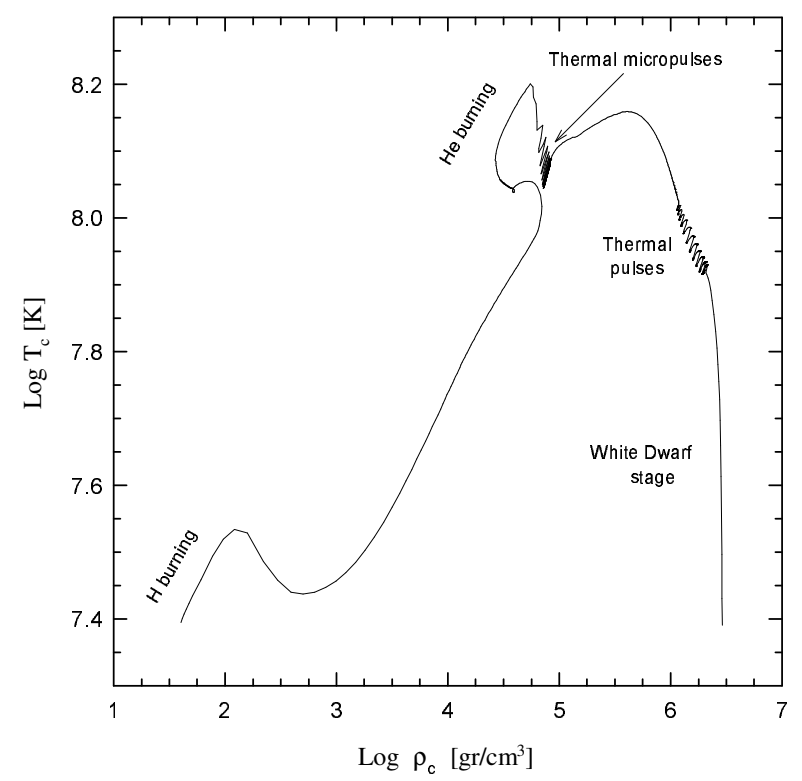

Figura 6.3: Temperatura central versus densidad central correspondiente a la evolución de nuestro modelo desde la ZAMS hasta la etapa de enana blanca. Los episodios relevantes en la evolución de la estrella tales como la combustión central de hidrógeno y helio, micropulsos y pulsos están también indicados.

en una "vuelta hacia el rojo" en el HRD. Durante esta fase la evolución ocurre lentamente ${ }^{2}$. Como resultado de la pérdida de masa la masa total del modelo se reduce hasta $0.563 \mathrm{M}_{\odot}$. El remanente alcanza finalmente la rama de enfriamiento de enana blanca. De ahí en adelante la difusión de elementos modifica la distribución de los elementos químicos en las regiones internas de la estrella, aún para los valores de $T_{\text {eff }}$ más bajos que se han calculado. Durante la etapa de enfriamiento de la enana blanca, los efectos acoplados de la combustión de hidrógeno y de la difusión de elementos reducen la masa de hidrógeno que queda en las capas externas en un factor de casi 2 .

La dependencia temporal de la luminosidad superficial durante la fase de los pulsos térmicos se detalla en el panel superior de la figura 6.2, donde la escala temporal está dada en Myr contados desde la ZAMS. Un total de 11 pulsos térmicos han sido computados antes de que el progenitor de enana blanca se aparta de la AGB. Esta fase de la evolución ha sido estudiada anteriormente por numerosos autores (Schönberner 1979; Iben 1982; Iben \& Renzini 1983; Vassiliadis \& Wood 1993 entre otros) y referimos al lector a esos estudios para mayores detalles. Sin embargo es interesante mencionar el rol que desempeñan las diferentes luminosidades relevantes en el momento en que el modelo evoluciona apartándose de la AGB hacia el dominio de las enanas blancas. Para esto se muestra en la figura 6.2 (panel inferior) la evolución de la luminosidad superficial $L_{*}$, luminosidad por combustión del helio $L_{H e}$ y luminosidad por combustión del hidrógeno $L_{H}$ durante los pulsos 10mo. y 11avo. El apartamiento del modelo de la AGB puede, en principio, ocurrir en diferentes estados durante el pulso dependiendo de

\footnotetext{
${ }^{2}$ No hemos considerado pérdida de masa adicional una vez que la estrella alcanza log $\mathrm{T}_{\text {eff }}=3.8$ por primera vez después de dejar la AGB.
} 
los detalles de la pérdida de masa.

En este trabajo nos concentramos en el caso en que la estrella deja la AGB durante la combustión estable del helio después del 11avo. pulso térmico. De esta manera, la simulación es representativa de aquellos casos en que la estrella deja la AGB temprano en el ciclo de los flashes de helio. La consecuencia para la evolución post-AGB resultante del apartamiento de la AGB en diferentes momentos durante el ciclo de los pulsos ha sido explorado por diversos investigadores (Iben, 1984; Wood \& Faulkner, 1986; Blöcker, 1995b). Estos estudios muestran, por ejemplo, que el tiempo de transición desde la AGB a la región de nebulosa planetaria depende fuertemente de la fase en la cual la estrella deja la AGB. En el caso estudiado aquí la combustión del helio contribuye con un $82 \%$ a la luminosidad superficial en el momento en que la estrella se aparta de la AGB, cuando la $\mathrm{T}_{\text {eff }}$ comienza a aumentar. En esos momentos la combustión del hidrógeno está prácticamente detenida.

En la figura 6.3 se muestra, por completitud, el comportamiento de las condiciones centrales de la estrella durante toda la evolución desde la ZAMS hasta la etapa de enana blanca. Los episodios relevantes en la vida de la estrella se indican en la figura. En particular, la respuesta de la región central a la ocurrencia de los micropulsos y pulsos térmicos puede verse claramente. Una vez que el remanente deja la AGB, la evolución procede a densidad prácticamente constante, formando una enana blanca.

\subsubsection{Evolución post-AGB y enana blanca}

Después del final de la fase de los pulsos térmicos, la estrella remanente se aparta de la AGB a bajas $\mathrm{T}_{\text {eff }} \mathrm{y}$ evoluciona como núcleo de nebulosa planetaria hacia $\mathrm{T}_{\text {eff }}$ altas. En la figura 6.4 se muestra como función de la $\mathrm{T}_{\text {eff }}$ la masa de hidrógeno remanente en las capas externas para el modelo post-AGB resultante de $0.563 \mathrm{M}_{\odot}$ desde un estadio previo a la finalización de la pérdida de masa hasta el correspondiente al régimen de estrella ZZ Ceti, en la rama de enfriamiento de enana blanca. Hacia el final de la pérdida de masa $\left(\log \mathrm{T}_{\text {eff }}=3.8\right)$ la contribución de la combustión de helio a la luminosidad total de la estrella se ha reducido a un $55 \%$. En ese momento el modelo experimenta un retorno a la AGB y aumenta su luminosidad hasta alcanzar el máximo valor en el interpulso. Durante esta fase la combustión del helio se vuelve menos importante y el contenido de hidrógeno de las capas externas es reducido, como resultado del aumento de la combustión del hidrógeno, desde $\approx 2 \times 10^{-3} \mathrm{M}_{\odot}$ al final de la pérdida de masa hasta $\approx 5 \times 10^{-4} \mathrm{M}_{\odot}$ una vez que el remanente reinicia su evolución hacia el azul. Para el momento en el cual la masa de hidrógeno decrece por debajo de $\approx 8 \times 10^{-4} \mathrm{M}_{\odot}$, la combustión del helio se vuelve prácticamente nula y la combustión del hidrógeno a través del ciclo CNO pasa a ser la fuente de energía dominante en la estrella. La mayor parte del tiempo de la transición post-AGB transcurre en esta etapa, durante la cual la evolución transcurre lentamente. En efecto, esta etapa dura aproximadamente 46000 años. Es importante notar que la estrella demora 48000 años en alcanzar una $\mathrm{T}_{\text {eff }}$ de $30000 \mathrm{~K}$, temperatura necesaria para la excitación de la nebulosa planetaria. Este tiempo es demasiado largo para que la nebulosa planetaria sea producida. Estos resultados son similares a los encontrados por Mazzitelli \& D'Antona (1986), quienes encuentran que si el apartamiento de la AGB tiene lugar durante la combustión estable del helio, entonces cuando la pérdida de masa es detenida la estrella pasa un tiempo considerable consumiendo la mayor parte de su envoltura de hidrógeno como super gigante roja. Wood \& Faulkner (1986) también encuentran tiempos evolutivos largos en la fase post-AGB cuando sus modelos abandonan la AGB temprano en la fase interpulso. Como es bien sabido, el tiempo transcurrido en la fase post-AGB es fuertemente dependiente de la fase 


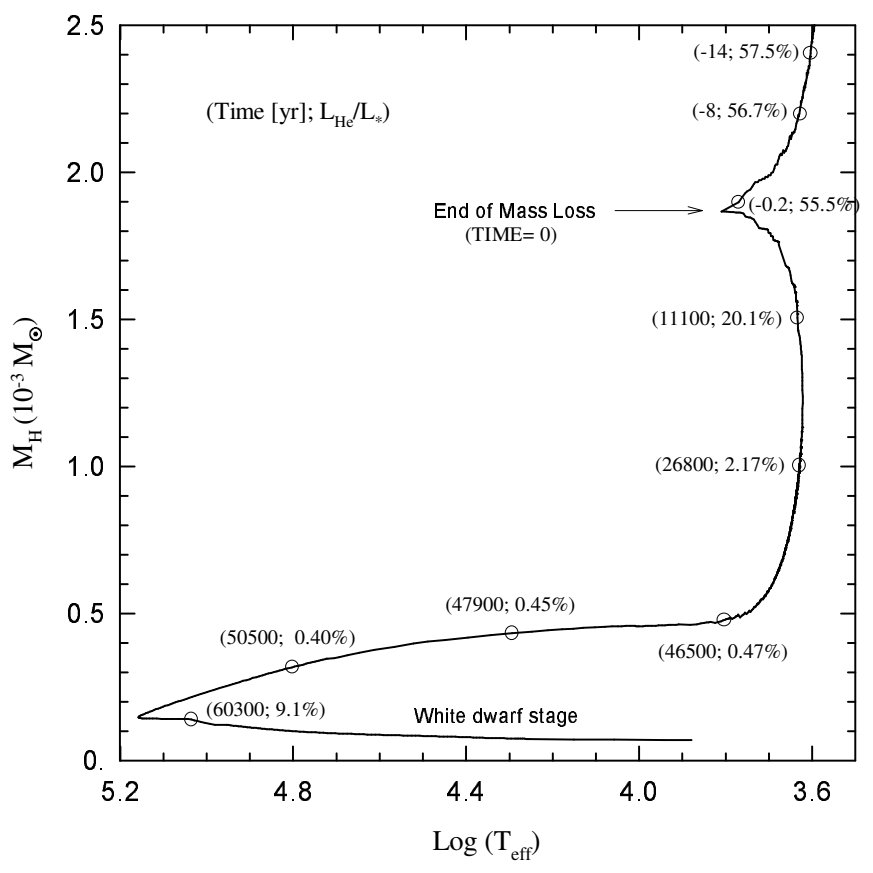

Figura 6.4: Masa de hidrógeno en las capas externas en unidades de $10^{-3}$ masas solares como función de la $\mathrm{T}_{\text {eff }}$ para las etapas evolutivas que preceden inmediatamente a la finalización de la pérdida de masa hasta el régimen de enana blanca. Los resultados corresponden al modelo remanente de $0.563 \mathrm{M}_{\odot}$. Los números en paréntesis a lo largo de la curva son la edad (en años) contada desde la finalización de la pérdida de masa y el porcentaje de la contribución de la combustión del helio a la luminosidad superficial del modelo. Notar que cuando la pérdida de masa es detenida la estrella retorna hacia bajas $\mathrm{T}_{\text {eff }}$ donde durante un intervalo de 40000 años la combustión nuclear reduce la masa de hidrógeno en casi un factor de 4. La combustión de helio se vuelve despreciable y el remanente reinicia su evolución hacia $\mathrm{T}_{\text {eff }}$ altas, alcanzando finalmente la fase de enana blanca con un contenido de hidrógeno de $\approx 1.5 \times 10^{-4} \mathrm{M}_{\odot}$. La combustión subsiguiente de hidrógeno reduce la envoltura residual de hidrógeno en un factor 2 adicional antes de alcanzar el dominio de las estrellas ZZ Ceti.

en el ciclo de helio en que la estrella abandona la AGB. En este sentido, si nuestro modelo hubiera sido forzado a dejar la AGB un poco después, durante la fase en que la combustión de hidrógeno representa la contribución principal a la luminosidad superficial (empleando por ejemplo una tasa de pérdida de masa menor), entonces su evolución posterior hubiera sido mucho más rápida (ver Mazzitelli \& D'Antona 1986; Wood \& Faulkner 1986). Finalmente es importante notar que la masa de hidrógeno es de $\approx 1.5 \times 10^{-4} \mathrm{M}_{\odot}$ al entrar en la rama de enfriamiento y es reducida $\mathrm{a} \approx 7 \times 10^{-5} \mathrm{M}_{\odot}$ en el momento en que la estrella entra en el dominio de las estrellas ZZ Ceti. Debido a que no hemos invocado pérdida de masa adicional durante la fase de nebulosa planetaria o durante las etapas tempranas en la rama de enfriamiento, el valor encontrado para la masa de hidrógeno debe ser considerado como un límite superior. El valor encontrado para la masa de hidrógeno es también dependiente de la metalicidad asumida en el modelo. Un valor menor de la metalicidad produce envolturas finales más masivas para una masa estelar dada (ver por ejemplo Iben \& MacDonald 1986). Un resultado equivalente se encuentra en el caso de enanas blancas con núcleos de helio (ver Serenelli, Althaus, Rohrmann \& Benvenuto 2002 y resultados del capítulo 5).

En el contexto de este trabajo es importante la estratificación química interna de la enana 
blanca. Por este motivo detallamos a continuación los perfiles químicos del remanente postAGB. En particular la distribución de las abundancias de carbono y oxígeno del remanente se muestran en la figura 6.5. La parte interna del núcleo de carbono y oxígeno resulta de la combustión del núcleo convectivo de helio y de las etapas posteriores en que el shell de helio se propaga hacia el exterior a medida que es consumido. Notar que este tipo de perfiles es característico, aparte de las diferencias originadas en el empleo de diferentes rates para la reacción ${ }^{12} \mathrm{C}(\alpha, \gamma){ }^{16} \mathrm{O}$, de los modelos en que ni semiconvección ni overshooting son considerados. El grado en que estos dos procesos afectan la estratificación química del núcleo es un tema de intenso debate y en la mayor parte de los estudios al respecto el tratamiento dado a estos procesos es muy rudimentario. Aquí preferimos no incluir ninguno de ellos y esto debe ser tenido en cuenta al considerar los resultados aquí presentados. Simplemente mencionaremos que en caso de incluir alguno o los dos procesos en los cálculos se esperan mayores abundancias de oxígeno en el núcleo ya que hacia el final de la etapa de combustión de helio en el núcleo, helio fresco de las capas superiores será transportado hacia el mismo y su combustión ocurrirá preferentemente a través de la reacción ${ }^{12} \mathrm{C}(\alpha, \gamma){ }^{16} \mathrm{O}$ en vez de la $3 \alpha$ (ver Mazzitelli \& D'Antona (1986) para más detalles). Antes de que la estrella alcance la configuración de enana blanca, un episodio de mezcla ocurre en las regiones centrales de la estrella. En efecto, debido a la forma particular del perfil de abundancias de carbono y oxígeno en $M_{r} / M_{*} \approx 0.2$ (líneas delgadas en figura 6.5), se desarrolla una inestabilidad Rayleigh-Taylor que da lugar a una rehomogeneización química de la zona más interna de la estrella (ver Salaris et al. 1997 para un resultado similar). Los perfiles resultantes después de la rehomogeneización se muestran en la misma figura en líneas gruesas y son los adoptados en este trabajo. Rodeando al interior de carbono-oxígeno hay un shell rico en carbono $(\sim 35 \%)$ y helio $(\sim 60 \%)$, y una capa exterior a este shell consistente prácticamente de helio puro, llamada buffer de helio. La presencia de carbono en la región rica en helio, por debajo del buffer de helio es resultado de la mezcla convectiva que lo ha transportado, desde la zona rica en carbono, hacia arriba durante el pico del último pulso térmico en la AGB. La masa del buffer de helio es de relevancia en conexión con la evolución subsiguiente del remanente durante la etapa de enana blanca, en particular con la ocurrencia de un flash de hidrógeno inducido por la difusión de elementos. En este sentido, Iben \& MacDonald (1986) han encontrado que si la masa del buffer de helio es tan pequeña como $0.001 \mathrm{M}_{\odot}$, entonces un flash de hidrógeno es iniciado como resultado de la difusión química. En nuestro modelo el buffer de helio tiene una masa de $3.4 \times 10^{-4} \mathrm{M}_{\odot}$ cuando la estrella deja la AGB, pero durante la evolución posterior en la cual el modelo retorna a la AGB y gran parte de la envoltura de hidrógeno es quemada, la masa del buffer aumenta hasta $0.0024 \mathrm{M}_{\odot}$ (y hasta $0.003 \mathrm{M}_{\odot}$ al iniciarse la etapa de enana blanca). El buffer de helio resulta entonces demasiado masivo como para que el flash de hidrógeno se produzca. Finalmente mencionemos que el contenido total de helio en la estrella, una vez que la combustión de helio se ha extinguido, es de $0.014 \mathrm{M}_{\odot}$.

La dependencia temporal de la contribución a la luminosidad causada por la combustión del hidrógeno a través de las reacciones de la cadena protón-protón $\left(L_{p p}\right)$ y del ciclo CNO $\left(L_{C N O}\right)$, de la combustión del helio $\left(L_{H e}\right)$, pérdidas por neutrinos $\left(L_{\nu}\right)$ y la luminosidad superficial $\left(L_{*}\right)$ para el modelo de enana blanca de $0.563 \mathrm{M}_{\odot}$ remanente se muestran en el panel superior de la figura 6.6, desde la etapa post-AGB hasta la luminosidad más baja computada. En el panel inferior se muestran las contribuciones relativas de $L_{H e}$ y $L_{H}=L_{p p}+L_{C N O}$ a la luminosidad superficial de la estrella. Además se muestra el cociente $L_{p p} / L_{H}$. Puede verse que al principio el ciclo CNO contribuye apreciablemente a la luminosidad superficial de la estrella. Después de $7 \times 10^{3}$ años de evolución (poco después de que el remanente alcance el punto de mayor $\mathrm{T}_{\text {eff }}$ ), las reacciones del ciclo CNO cesan abruptamente, y el modelo comienza a descender por la 


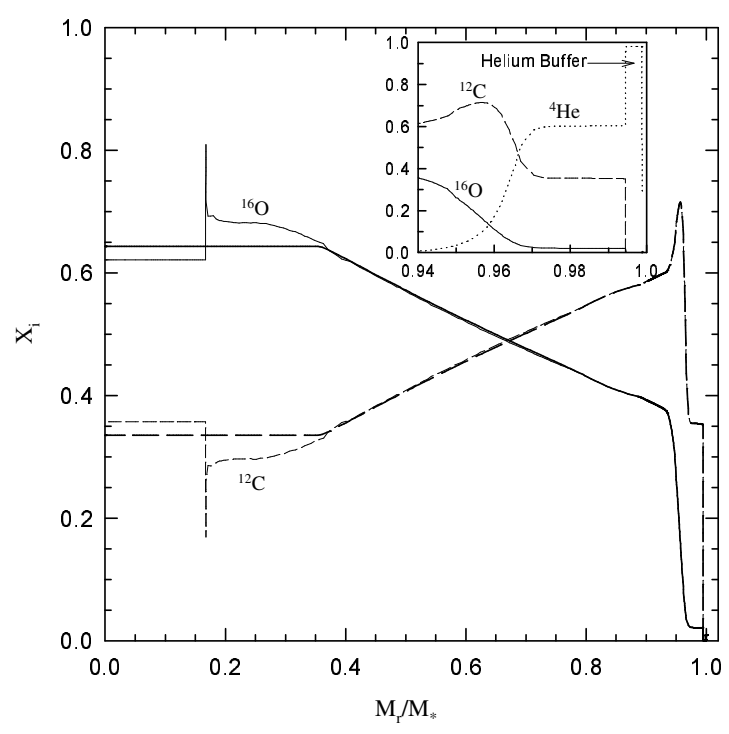

Figura 6.5: Perfiles de abundancia de carbono y oxígeno en el núcleo del remanente de $0.563 \mathrm{M}_{\odot}$ enseguida después de la finalización de la pérdida de masa. Las líneas gruesas denotan los perfiles después de la rehomogeneización causada por una inestabilidad de Rayleigh-Taylor que ocurre en la región central de la estrella. Las líneas delgadas muestran los mismos perfiles antes de la rehomogeneización. El gráfico insertado muestra la distribución de abundancias del helio, carbono y oxígeno en las capas externas de la estrella.

rama de enfriamiento, en el dominio de las enanas blancas, cuando la luminosidad superficial comienza a disminuir rápidamente. En ese momento $L_{H e}$ contribuye casi tanto como $L_{H}$ a la luminosidad superficial. De ahí en más, la combustión nuclear juega un rol menor en la evolución de la estrella, que está dominada por las pérdidas por neutrinos y por la liberación de energía gravotérmica. La contribución máxima de la combustión nuclear a la luminosidad superficial durante la fase de enana blanca ocurre en el rango de $\mathrm{T}_{\text {eff }}$ entre 35000 y $20000 \mathrm{~K}$ (desde $5 \times 10^{6}$ a $4 \times 10^{7}$ años de evolución). Durante esta fase de la evolución la combustión nuclear ocurre principalmente a través del ciclo CNO. A una edad de $\approx 10^{8}$ años la producción de energía por el ciclo CNO cae por debajo de la producida por la cadena protón-protón. En el dominio de las ZZ Ceti es esta cadena de reacciones la única que contribuye apreciablemente como fuente de energía de la estrella. Es interesante destacar que durante esta etapa el cociente luminosidad nuclear/superficial alcanza un máximo local. En el borde azul de la banda de inestabilidad de las ZZ Ceti la luminosidad nuclear es de alrededor de un $10 \%$ de la superficial, mientras que en el borde rojo es de un 5\% aproximadamente. A medida que la evolución continua a luminosidades y $\mathrm{T}_{\text {eff }}$ más bajas la luminosidad nuclear se vuelve prácticamente despreciable.

Una vez que la estrella ha alcanzado la rama de enfriamiento, la distribución de su composición química es fuertemente modificada por los procesos de difusión que actúan durante la evolución de la enana blanca. Para ilustrar esto se puede ver en la figura 6.7 las abundancias por masa de ${ }^{1} \mathrm{H},{ }^{3} \mathrm{He},{ }^{4} \mathrm{He},{ }^{12} \mathrm{C}$ y ${ }^{14} \mathrm{~N}$ como función de la fracción de masa externa $q$ ( $q=1-M_{r} / M_{*}$ de modo que el centro de la estrella corresponde a $\left.\log q=0\right)$ para diferentes momentos en la evolución, caracterizados por valores de $\log L / \mathrm{L}_{\odot} \mathrm{y} \log \mathrm{T}_{\text {eff }}$ (números dados en paréntesis). Además la liberación de energía nuclear $\epsilon_{n u c}$ causadas por las combustiones de 

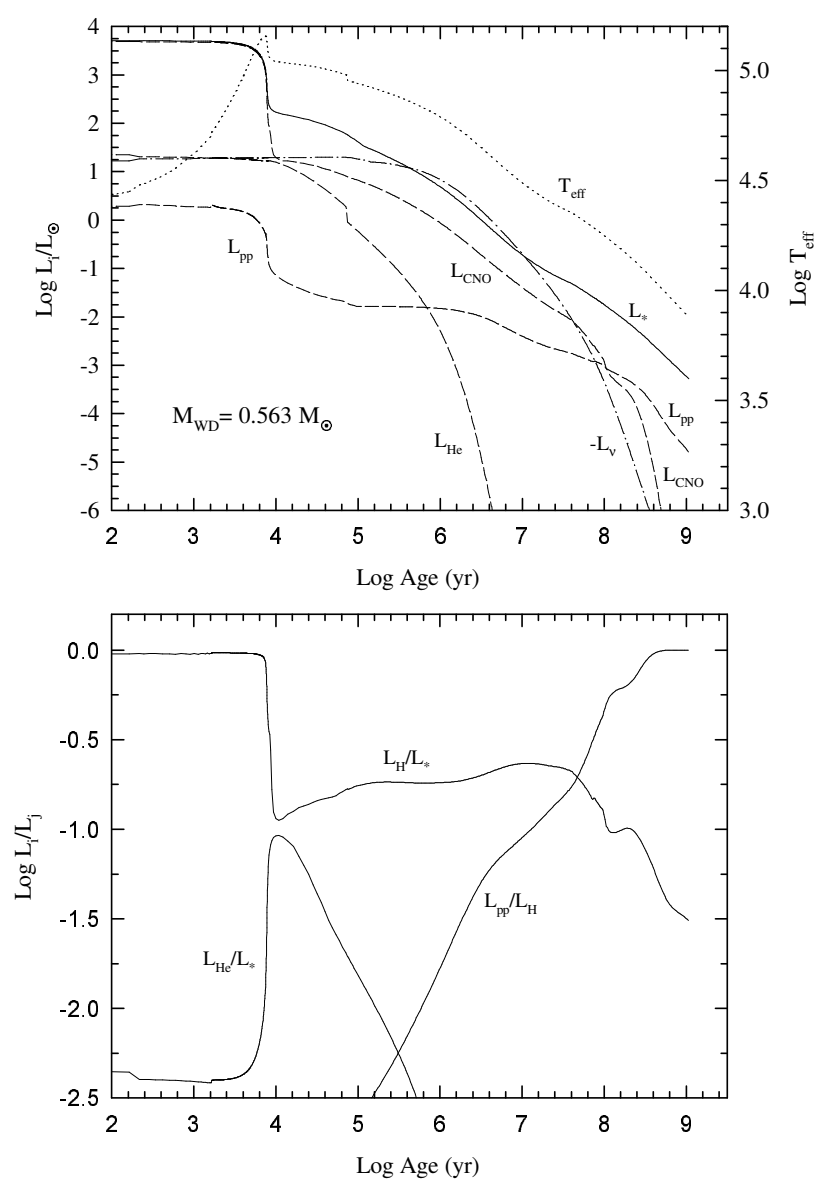

Figura 6.6: El panel superior muestra las diferentes contribuciones a la luminosidad del modelo (en unidades solares) como función del tiempo: luminosidad superficial $\left(L_{*}\right)$, por cadena protón-protón $\left(L_{p p}\right)$, por ciclo CNO $\left(L_{C N O}\right)$, por combustión del helio $\left(L_{H e}\right)$ y por pérdida por neutrinos $\left(L_{\nu}\right)$. La evolución de la $\mathrm{T}_{\text {eff }}$ también se muestra. Aquí el punto de edad cero corresponde al momento en que el modelo alcanza el punto donde $\log \mathrm{T}_{\text {eff }}=4.40$. El panel inferior muestra los cocientes de luminosidades. $L_{H}$ es la luminosidad total por combustión de hidrógeno $\left(L_{H}=L_{p p}+L_{C N O}\right)$. Notar que cuando el dominio de las estrellas ZZ Ceti es alcanzado, $L_{H}$ (que es dominado por $L_{p p}$ ) alcanza un máximo, con una contribución a la luminosidad superficial de un $10 \%$.

hidrógeno y helio se muestran también en líneas delgadas. El panel a muestra la estratificación química antes de que la estrella alcance el punto de máxima $T_{\text {eff }}$ a altas luminosidades. En las capas externas las abundancias corresponden escencialmente a las abundancias iniciales asumidas para el modelo (abundancias solares). En el buffer de helio las abundancias de ${ }^{12} \mathrm{C} \mathrm{y}{ }^{14} \mathrm{~N}$ reflejan las abundancias resultantes del ciclo $\mathrm{CNO}$, diferentes de las solares, habiéndose transformado casi todo el ${ }^{12} \mathrm{C}$ en ${ }^{14} \mathrm{~N}$. El panel $b$ corresponde a un modelo al comienzo de la rama de enfriamiento, $5 \times 10^{5}$ años después de la finalización de la pérdida de masa. Los efectos de la difusión son sólo apreciables en las capas externas, donde la sedimentación gravitatoria hace que los elementos pesados se hundan. Los cambios observados en la zona del buffer de helio se deben principalmente a la combustión nuclear, a través del ciclo CNO en la base de la envoltura de hidrógeno. Cuando la estrella evoluciona a lo largo de la curva de enfriamiento (paneles $c$ y d), la acción de la sedimentación gravitatoria se hace evidente, dando lugar a envolturas puras de hidrógeno. También es notorio el efecto de la difusión química donde existen gradientes de 

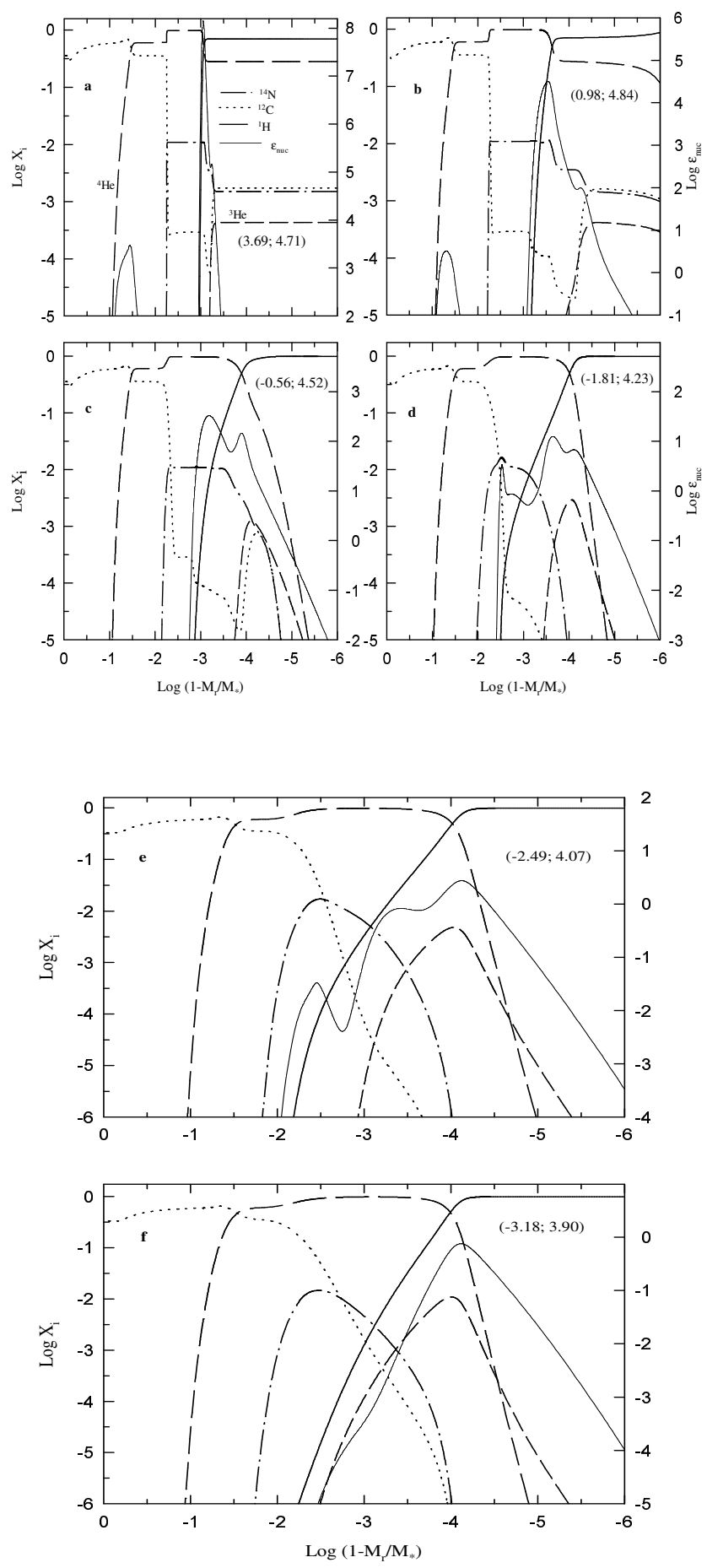

Figura 6.7: Abundancia por masa de ${ }^{1} \mathrm{H},{ }^{3} \mathrm{He},{ }^{4} \mathrm{He},{ }^{12} \mathrm{C}$ y ${ }^{14} \mathrm{~N}$ como función de la fracción de masa externa para el modelo de enana blanca en diferentes puntos de su evolución, caracterizados por valores de $\log L / \mathrm{L}_{\odot} \mathrm{y} \log \mathrm{T}_{\text {eff }}$ (números dados en paréntesis). También se muestra la tasa de liberación de energía nuclear $\epsilon_{n u c}$ (en erg $\mathrm{g}^{-1} \mathrm{~s}^{-1}$ ) en línea delgada (la combustión del helio sólo es relevante en los modelos $a$ y $b$ ). El modelo mostrado en $a$ corresponde a un punto antes de alcanzar el momento de máxima $\mathrm{T}_{\text {eff }}$ a altas luminosidades mientras que el mostrado en $f$ corresponde al último modelo calculado. En $e$ se muestra un modelo correspondiente a la fase de ZZ Ceti. Notar en este modelo la extensión de la cola interna del perfil de hidrógeno, así como la extensión de la zona donde se produce la liberación de energía nuclear. Se aprecia claramente en estos gráficos la influencia de los procesos de difusión en los perfiles de abundancias durante la evolución de la enana blanca. 
abundancias grandes. Como resultado de esto puede verse que en el buffer de helio existe una cola de la distribución de hidrógeno a edades más avanzadas (panel $d$ ) y una de la distribución del carbono en la parte interna del mismo. La cola de hidrógeno que se difunde hacia el interior es la que favorece la liberación de energía nuclear ya que el hidrógeno alcanza de esta manera regiones de temperaturas más elevadas. La estratificación química cuando el modelo alcanza la región de las ZZ Ceti puede verse en el panel $e$, cuando la edad de enfriamiento es de $3 \times 10^{8}$ años. Los perfiles de abundancias han sido notoriamente modificados con respecto a los correspondientes al inicio de la rama de enfriamiento. En particular la estrella está caracterizada por una envoltura pura de hidrógeno de $3.5 \times 10^{-5} \mathrm{M}_{\odot}$ más una cola de hidrógeno ancha, que alcanza capas bien dentro de la estrella (la masa total de hidrógeno es $\left(7 \times 10^{-5} \mathrm{M}_{\odot}\right)$. En este momento la cola de hidrógeno alcanza su máxima profundidad. También es notoria la cola de carbono, que se extiende desde el interior de la estrella hacia el buffer de helio. A medida que la estrella se siga enfriando la cola de hidrógeno comienza a retraerse hacia el exterior debido al aumento de la degeneración electrónica, que causa que la difusión química se vuelva menos importante, deteniendo la difusión del hidrógeno. El perfil químico correspondiente al último modelo calculado se muestra en el panel $f$. Comparando los paneles $e$ y $f$ resulta evidente que, a excepción de las capas más externas, el equilibrio difusivo no ha sido alcanzado en estas etapas. Esto permite afirmar que la aproximación de equilibrio difusivo, usualmente empleada para determinar los perfiles químicos de las enanas blancas DA en los estudios pulsacionales de las mismas no resulta apropiado. Otro punto interesante concerniente a la difusión en la distribución de los elementos está relacionado con la evolución de las abundancias en los perfiles de ${ }^{12} \mathrm{C}$ y ${ }^{14} \mathrm{~N}$ en el buffer de helio. Inicialmente (panel $a$ ) puede verse que en el buffer de helio el ${ }^{14} \mathrm{~N}$ es mucho más abundante que el ${ }^{12} \mathrm{C}$; sin embargo en las últimas etapas computadas el ${ }^{12} \mathrm{C}$ resulta más abundante que el ${ }^{14} \mathrm{~N}$ en casi todo el interior estelar (a excepción de una parte del buffer de helio), aún cuando el ${ }^{12} \mathrm{C}$ ha sido procesado en ${ }^{14} \mathrm{~N}$ por la combustión nuclear durante la evolución como enana blanca. Un resultado similar ha sido encontrado por Iben \& MacDonald (1986) aunque en sus cálculos el ${ }^{12} \mathrm{C}$ es más abundante que el ${ }^{14} \mathrm{~N}$ en el buffer de helio cuando la estrella alcanza el dominio de las ZZ Ceti. Esta diferencia puede deberse en parte a que los cálculos de Iben \& MacDonald (1986) no incluyen la difusión térmica. Dado que ésta actúa en la misma dirección que la sedimentación gravitatoria, el no tenerla en cuenta lleva a mayores abundancias de ${ }^{12} \mathrm{C}$ en el buffer de helio. En este contexto, este efecto puede ser responsable de diferencias en la luminosidad por combustión nuclear con respecto al caso en que la difusión térmica es tenida en cuenta. Para verificar esta afirmación se ha realizado un cálculo en el cual la difusión térmica no ha sido tenida en cuenta. En ese caso la abundancia de ${ }^{12} \mathrm{C}$ encontrada en el buffer de helio al comienzo del dominio de las ZZ Ceti es mayor que en el caso que incluye difusión térmica, y además la contribución de la luminosidad nuclear con respecto a la luminosidad superficial alcanza hasta un $20 \%$.

Un aspecto que merece también ser discutido es el efecto de la difusión química en la combustión nuclear. En el caso de un modelo de $Z=0.001$ ha sido discutido por Iben \& MacDonald (1985). La situación para el caso $Z=0.02$ es cualitativamente similar aunque existen algunas diferencias. La difusión hace que las reacciones del ciclo CNO sean eficientes durante un lapso de tiempo mayor que cuando la difusión no es tenida en cuenta. Esto puede verse en la figura 6.7 mirando la distribución de la energía nuclear generada dentro de la estrella. Debido a la cola de hidrógeno que se difunde hacia adentro en el buffer de helio, y al carbono difundiéndose desde adentro hacia el exterior penetrando también en el buffer de helio, la producción de energía nuclear vía las reacciones del ciclo CN son significativas por un largo período de tiempo en la evolución de la enana blanca. La ubicación del máximo de 


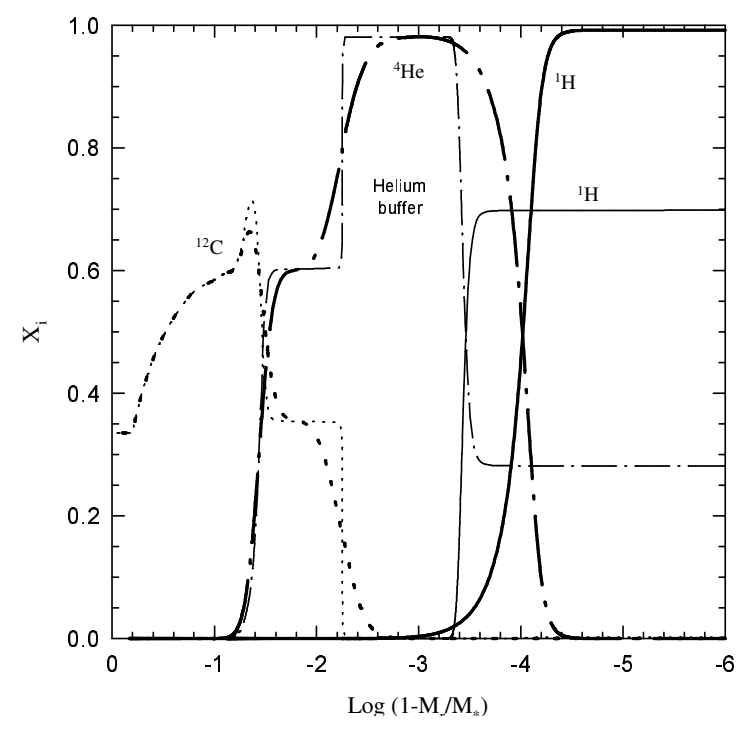

Figura 6.8: Perfiles de abundancia del remanente enana blanca para dos modelos seleccionados justo después del punto de máxima $\mathrm{T}_{\text {eff }}$ (líneas delgadas) y cerca del comienzo del régimen de ZZ Ceti (líneas gruesas). El efecto de la difusión es claramente notable, en particular nótese cómo los gradientes de abundancias son suavizados por efectos de la difusión química, mientras que la sedimentación gravitatoria produce una envoltura pura de hidrógeno.

$\epsilon_{C N}$ se vuelve más profunda a medida que la evolución procede, alcanzando una profundidad máxima de $\approx 1.7 \times 10^{-3} \mathrm{M}_{\odot}$ por debajo de la superficie estelar. Notar que a pesar de que la difusión produce abundancias aumentadas de hidrógeno y carbono en el buffer de helio, esto no resulta en un flash termonuclear. Esta situación podría ser diferente si el buffer de helio hubiera sido menos masivo que el obtenido en nuestros cálculos $\left(0.003 \mathrm{M}_{\odot}\right)$. En efecto, Iben \& MacDonald (1986) mostraron que un flash de hidrógeno se inicia cuando la estrella se encuentra en la rama de enfriamiento si el buffer de helio es tan pequeño como $0.001 \mathrm{M}_{\odot}$, y que éste es evitado cuando si el buffer tiene $0.005 \mathrm{M}_{\odot}$. Como se mencionó antes, cuán masivo es el buffer de helio depende (para un progenitor de masa dada) de la fase en el ciclo de los flashes de helio durante los pulsos térmicos en que la estrella deja la AGB. Por lo tanto, el rol de las reacciones nucleares, en particular debido al ciclo $\mathrm{CN}$, depende también del momento en que la estrella deja la AGB. Finalmente, también puede verse en la figura 6.7 que a medida que la estrella se enfría, el rango de masa en el cual ocurre la combustión nuclear del hidrógeno se vuelve más ancho a medida que la cola de la distribución de hidrógeno penetra hacia adentro. Puede verse que el máximo ancho se alcanza en la región de las ZZ Ceti, por lo que la estabilidad de los modos en los modelos de enana blanca DA podría ser afectada por la ocurrencia de combustión nuclear en un rango de masa considerable. En este sentido, hay que mencionar que la combustión nuclear en un shell, a través del mecanismo- $\epsilon$, es responsable de la inestabilidad de los modos-g en modelos de pre-enana blanca (Kawaler et al., 1986; Kawaler, 1988). Sin embargo, los períodos de los modos observados son tan pequeños que no han sido observados en dichas estrellas (Hine \& Nather, 1987). De todas formas, nuestros cálculos indican que la combustión de hidrógeno contribuye a lo sumo con un $10 \%$ a la luminosidad superficial durante la etapa ZZ Ceti. La combustión nuclear no representa en el caso de nuestros modelos una fuente importante de energía aún para la envoltura de hidrógeno más masiva predicha por la evolución pre-enana blanca. Esto es consecuencia no sólo del hecho de que nuestros cálculos 
asumen una metalicidad alta, $Z=0.02$ (ver Iben \& MacDonald 1986), sino que también se debe a la inclusión de la difusión térmica que, como se ha dicho, disminuye la contribución de la combustión del hidrógeno a la luminosidad superficial.

La forma de los perfiles de composición química es un factor clave para determinar los períodos de los modos-g de las estrellas DAV. En la figura 6.8 comparamos la estratificación química de la enana blanca en el dominio de inestabilidad con el correspondiente al comienzo de la rama de enfriamiento. El rol de la difusión es notorio en esta figura. Las casi discontinuidades iniciales, resultantes de los episodios de convección durante la evolución del progenitor en la AGB, son suavizadas notablemente por la difusión. En efecto, la difusión modifica fuertemente la pendiente de los perfiles químicos en las regiones externas de la estrella a lo largo de la rama de enfriamiento. Como es bien sabido, tales regiones resultan críticas para las propiedades pulsacionales de las enanas blancas. En la próxima sección describimos los efectos de estos perfiles en las cantidades principales que entran en las ecuaciones de pulsaciones adiabáticas. Un análisis detallado del espectro pulsacional de las DAV está más allá del alcance de este trabajo y referimos al lector a Córsico, Althaus, Benvenuto \& Serenelli (2001, 2002); Córsico (2002).

\subsection{Cantidades para el análisis de las pulsaciones adiabáticas}

Aquí se describen las características de las variables básicas que son relevantes para el análisis de las pulsaciones adiabáticas. Nos concentramos particularmente en la frecuencia de BruntVäisälä (BVF) y el término de Ledoux (B). Para realizar el análisis elegimos nuestro modelo correspondiente a una $\mathrm{T}_{\text {eff }}=12000 \mathrm{~K}$, dentro de la banda de inestabilidad de las ZZ Ceti.

En términos generales, la BVF es la frecuencia de oscilación de una porción de fluido estelar estratificada cuando es desplazada verticalmente (radialmente) de su posición de equilibrio, y la flotación actúa como fuerza restauradora. La BVF $(N)$ se define como (Unno et al., 1989):

$$
N^{2} \equiv g\left(\frac{1}{\Gamma_{1}} \frac{\mathrm{d} \ln P}{\mathrm{~d} r}-\frac{\mathrm{d} \ln \rho}{\mathrm{d} r}\right) .
$$

Como es bien sabido, la forma de la BVF es directamente responsable de las características globales del espectro de períodos en las enanas blancas pulsantes. Puede demostrarse empleando un análisis local (ver por ejemplo Unno et al. 1989) que la condición para modos-g que se propagan (no-evanescentes) es que la frecuencia al cuadrado, $\sigma^{2}$, debe ser menor que $L_{\ell}^{2}$ y que $N^{2}$ ( $L_{\ell}^{2}$ es la frecuencia acústica de Lamb, la otra frecuencia crítica en las oscilaciones no-radiales de las estrellas). La región del modelo donde esta condición es cumplida es la zona de propagación del modelo (ver diagramas de propagación en, por ejemplo, Cox 1980; Unno et al. 1989). En particular, para enanas blancas, la BVF alcanza valores muy pequeños dentro del núcleo degenerado, excluyendo la posibilidad de propagación de modos-g de orden bajo (períodos cortos). En su lugar, estos modos se propagan en la envoltura de la estrella, y por lo tanto son muy sensibles a los detalles de la estructura de las regiones externas.

Desde el punto de vista computacional, el tratamiento de la BVF en el interior de las estrellas enanas blancas ha sido ampliamente discutido previamente (ver Tassoul, Fontaine \& Winget 1990 y en particular Brassard et al. 1991). Estos estudios ilustran los problemas numéricos y errores sistemáticos que resultan del cálculo de la BVF cuando ésta es calculada directamente de su definición (ec. 6.1). Brassard et al. (1991) han mostrado que, en el marco 
de sus modelos estelares, el perfil de BVF obtenido de la ec. 6.1 puede llevar a un patrón no realista de períodos de modos-g. Como se menciona en ese artículo, la razón para ello no es el cálculo de las derivadas numéricas, sino el empleo directo de la ec. 6.1 para materia fuertemente degenerada, típica de los interiores de las enanas blancas, que implican la substracción de dos cantidades grandes que son casi iguales lo que produce, además de estructuras espúreas en el perfil de $N$, un corrimiento global hacia valores mayores de la BVF, particularmente para la parte interna del núcleo. Esto lleva a un aumento de las autofrecuencias de los modos-g o, en otras palabras, a un desplazamiento hacia períodos más cortos. Además, la región de formación del período, caracterizada por las funciones de peso (ver Brassard et al. 1991), es afectada fuertemente.

Para evitar estas dificultades, Brassard et al. (1991) proveen una estrategia computacional apropiada para objetos degenerados, conocida como tratamiento modificado de Ledoux. De acuerdo a estos autores, $N^{2}$ en las enanas blancas puede calcularse como

$$
N^{2}=\frac{g^{2} \rho \chi_{T}}{P \chi_{\rho}}\left(\nabla_{a d}-\nabla+B\right)
$$

donde $\chi_{T}\left(\chi_{\rho}\right)$ denota la derivada parcial logarítmica de la presión con respecto a $T(\rho), \nabla$ y $\nabla_{a d}$ son los gradientes de temperatura real y adiabático, respectivamente, y $B$ el término de Ledoux que está dado por

$$
B=-\frac{1}{\chi_{T}} \sum_{i=1}^{n-1} \chi_{X_{i}} \frac{\mathrm{d} \ln X_{i}}{\mathrm{~d} \ln P}
$$

donde $X_{i}$ es la abundancia por masa de las especies $i, n$ es el número total de especies consideradas y

$$
\chi_{X_{i}}=\left(\frac{\partial \ln P}{\partial \ln X_{i}}\right)_{\rho, T,\left\{X_{j \neq i}\right\}}
$$

Esta formulación tiene la ventaja de evitar los problemas mencionados anteriormente, y al mismo tiempo tiene en cuenta explícitamente la contribución a $N^{2}$ de cualquier cambio en la composición en el interior del modelo (zonas de transición química ) por medio del término de Ledoux. Brassard et al. (1992a) enfatizan la relevancia del correcto tratamiento de la BVF en las interfaces de composición química en enanas blancas estratificadas, particularmente en conección con los efectos de resonancia de modos, fenómeno conocido como "modos atrapados". El tratamiento modificado de Ledoux es empleado en la mayoría de los estudios pulsacionales de enanas blancas. El código de pulsaciones empleado en estos cálculos (Córsico \& Benvenuto, 2002) está también basado en esta formulación.

El término de Ledoux $B$ es un ingrediente importante en el cálculo de $N^{2}$. En la mayor parte de los estudios existentes, la forma de $B$ es calculada en base a perfiles químicos tratados en base al equilibrio difusivo en la aproximación elemento-traza (ver, por ejemplo, Tassoul, Fontaine \& Winget (1990); Brassard et al. (1991, 1992a,b)). El comportamiento de $B$ es responsable (a través de $N^{2}$ ) de efectos macroscópicos en la distribución de períodos en enanas blancas estratificadas, tales como los modos atrapados y el confinamiento de modos (siguiendo la terminología de Brassard et al. 1992b). En el panel superior de la figura 6.9 se muestran los perfiles de abundancias de las especies químicas más relevantes de nuestros modelos. En el panel central se ilustra el término $B$ correspondiente y en el panel inferior se muestra $N^{2}$. Los perfiles químicos predichos en nuestros cálculos son muy suaves en las interfaces, lo cual da 

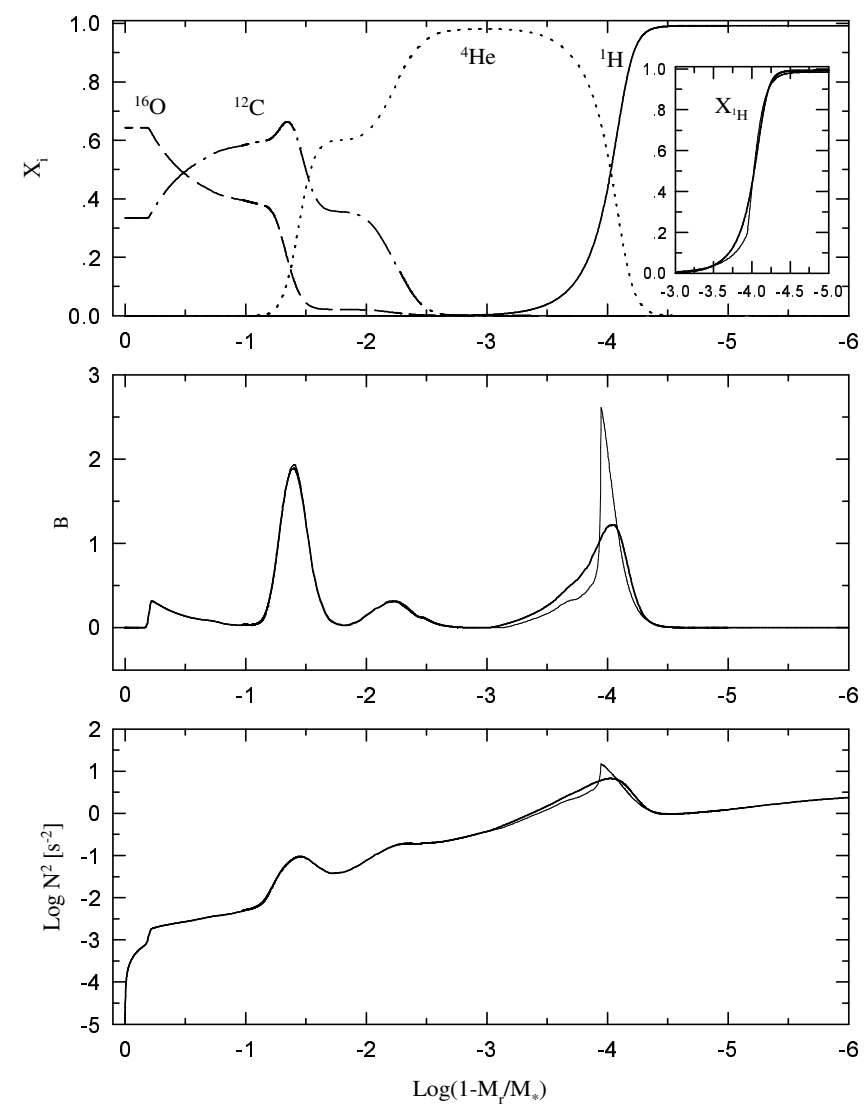

Figura 6.9: Panel superior: perfil químico interno de hidrógeno, helio carbono y oxígeno para un modelo de ZZ Ceti de $0.563 \mathrm{M}_{\odot}$ a $\mathrm{T}_{\text {eff }}=12000 \mathrm{~K}$. La interface de hidrógeno-helio se muestra en el gráfico pequeño insertado en el mismo panel, junto con los resultados correspondientes a la aproximación elemento-traza del equilibrio difusivo (línea delgada). Panel central: término de Ledoux $B$ correspondiente. Nuevamente la línea delgada corresponde a la aproximación de equilibrio difusivo. Panel inferior: logaritmo del cuadrado de la frecuencia de Brunt-Väisälä .

lugar a colas extendidas en la forma de $B$. También es importante notar que nuestro modelo está caracterizado por una interface química en la que coexisten tres especies químicas con abundancias apreciables: oxígeno, carbono y helio. Esta transición da dos contribuciones a $B$, una de ellas de magnitud relativamente grande, localizada en log $q \sim-1.4$, y otra más externa y pequeña en $\log q \sim-2.2$. Esta característica produce una diferencia en comparación a los resultados de otros autores (ver Tassoul, Fontaine \& Winget 1990; Brassard et al. 1991, 1992a,b; Bradley 1996). Por último, notar que la contribución de la interface H-He es menor que la correspondiente a la transición O-C-He. Del panel inferior de la figura 6.9 puede verse que cada rasgo de $B$ se refleja en la forma de BVF. Las contribuciones del término de Ledoux se transforman en prominencias extendidas en $N^{2}$. Notar el comportamiento global suave de estas cantidades cuando se emplean modelos evolutivos que incorporan la dependencia temporal en la difusión de los elementos. A los efectos de comparación, se incluyen en la figura los términos $B$ y $N^{2}$ calculados de acuerdo al equilibrio difusivo en la aproximación de elemento-traza dada por Tassoul, Fontaine \& Winget (1990). El perfil químico resultante en la transición H-He 

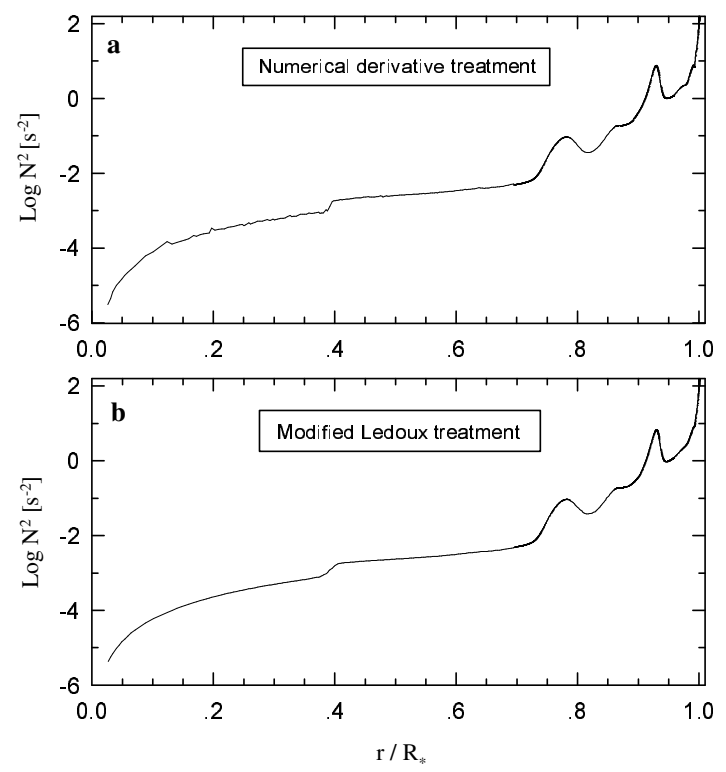

Figura 6.10: (a) Logaritmo del cuadrado de la frecuencia de Brunt-Väisälä como función del radio estelar usando derivadas numéricas en la ec. 6.5. (b) La misma cantidad pero calculada usando la ec. 6.2. El modelo estelar es el mismo de la figura 6.9 .

obtenido mediante esta aproximación se muestra en el panel superior de la figura 6.9 en líneas delgadas. De acuerdo con estudios previos, notar que la aproximación de equilibrio difusivo lleva a un pico pronunciado en el término de Ledoux en la interface química, que se traslada en un pico muy abrupto en esa región en $N^{2}$.

Por completitud, también hemos computado $N^{2}$ directamente de la ec. 6.1. Usando la condición de equilibrio hidrostático, la ec. 6.1 puede reescribirse como

$$
N^{2}=-\frac{g^{2} \rho}{P}\left(\frac{\operatorname{dln} \rho}{\operatorname{dln} P}-\frac{1}{\Gamma_{1}}\right) .
$$

La derivada $\frac{\operatorname{dln} \rho}{\operatorname{dn} P}$ en esta ecuación ha sido calculada nuéricamente empleando un esquema de interpolación apropiado. Este esquema proporciona la derivada primera en los puntos interpolados. El resultado para $N^{2}$ se muestra en la figura 6.10 (panel superior) en términos de $r / R_{*}$. Por comparación, en el panel inferior de la misma figura se muestra $N^{2}$ calculada de acuerdo al tratamiento modificado de Ledoux. Notar que la estrategia empleada para las derivadas numéricas da excelentes resultados, reproduciendo hasta los más finos detalles del perfil de $N^{2}$ calculada con la ec. 6.2. Hacia el centro, apenas pueden observarse estructuras muy pequeñas originadas por ruido numérico. En contraste con lo afirmado por Brassard et al. (1991), nuestros cálculos basados en un esquema interpolatorio apropiado proveen un perfil para $N^{2}$ confiable en nuestros modelos estelares (ver un resultado similar en Gautschy, Ludwig \& Freytag 1996).

\subsection{Conclusiones}

Se han presentado cálculos evolutivos de estrellas enanas blancas DA. Los cálculos tienen en cuenta la difusión de elementos incluyendo su dependencia temporal, la combustión nuclear y la 
historia del progenitor de la enana blanca de manera auto-consistente. La aplicación principal de estos modelos será la exploración de sus propiedades pulsacionales (objetivo fuera del alcance de esta tesis).

Específicamente, seguimos la evolución de una estrella de $3 \mathrm{M}_{\odot}$ inicialmente desde la ZAMS hasta el extremo de la AGB. Hacia el final de la combustión del helio en el núcleo la estrella experimenta unos 40 micropulsos de baja amplitud en la luminosidad superficial. Después la evolución continúa hacia la $\mathrm{AGB}$, donde la estrella experimenta los pulsos térmicos principales, causados por inestabilidades en el shell de helio. Después de 11 pulsos térmicos el modelo es forzado a evolucionar hacia la configuración de enana blanca aplicando una fuerte pérdida de masa al modelo. La evolución es seguida hasta el dominio de las ZZ Ceti, en la rama de enfriamiento de las enanas blancas.

Encontramos que si la estrella se aparta de la AGB temprano en el ciclo interpulso, durante la combustión estacionaria de helio, entonces después de la fase de pérdida de masa $\left(\log \mathrm{T}_{\text {eff }}=3.8\right)$, la estrella retorna al rojo donde la envoltura de hidrógeno es considerablemente reducida por la combustión del mismo en la base de la envoltura. Como resultado no se produce una nebulosa planetaria ya que el remanente necesita $\sim 48000$ años en alcanzar los 30000K, temperatura necesaria para la excitación de la nebulosa. Esto está de acuerdo con los resultados encontrados por Mazzitelli \& D'Antona (1986); Wood \& Faulkner (1986). La masa de hidrógeno que queda al comienzo de la rama de enfriamiento de enana blanca es de $\sim 1.5 \times 10^{-4} \mathrm{M}_{\odot}$, y es reducida a $7 \times 10^{-5} \mathrm{M}_{\odot}$ debido a los efectos combinados de la combustión nuclear y la difusión de elementos para el momento en que la estrella alcanza el dominio de las ZZ Ceti. Debido a que no hemos invocado pérdida de masa adicional durante la fase de núcleo de nebulosa planetaria o durante las fases tempranas en la rama de enfriamiento, el valor de masa dado para la envoltura de hidrógeno debe considerarse como una cota superior. Otra característica de interés es el tamaño del buffer de helio. Éste tiene $\approx 3 \times 10^{-4} \mathrm{M}_{\odot}$ cuando la estrella deja la AGB, pero cuando el remanente retorna hacia el rojo, la masa del buffer aumenta hasta $0.0024 \mathrm{M}_{\odot}$, el cual es lo suficientemente masivo como para prevenir un flash de hidrógeno inducido por la difusión. De esta manera, concluimos que si la estrella se aparta de la AGB cuando el combustión de helio ocurre de manera estacionaria, no es posible la ocurrencia de una nova auto-inducida como predicen Iben \& MacDonald (1986).

De acuerdo con Iben \& MacDonald (1985) encontramos que la difusión de elementos modifica fuertemente la distribución de las abundancias químicas durante el enfriamiento de la enana blanca. Las discontinuidades en las distribuciones de abundancias, presentes al comienzo de la rama de enfriamiento, son suavizadas considerablemente por los procesos de difusión al momento en que el dominio de ZZ Ceti es alcanzada. Nuestros cálculos muestran, además, que el equilibrio difusivo no es alcanzado (excepto en las capas externas) cuando la estrella alcanza el dominio de las ZZ Ceti. Durante la fase de ZZ Ceti se observa una evolución apreciable de las abundancias químicas. Con respecto a las reacciones nucleares, encontramos que no representan una fuente importante de energía durante la etapa de enana blanca, como se espera para una estrella progenitora de alta metalicidad. Encontramos también que la difusión térmica disminuye la importancia de las reacciones nucleares.

En cuanto a las cantidades relevantes en el cálculo de las pulsaciones adiabáticas, encontramos que la forma del término de Ledoux es marcadamente diferente del encontrado en estudios previos de pulsaciones de enanas blancas. Esto es debido en parte al efecto de suavizado de los perfiles químicos causado por la difusión de elementos, lo que da lugar a picos menos pronunciados en el término de Ledoux y la frecuencia de Brunt-Väisälä.

Esperamos que las propiedades pulsacionales de los modelos evolutivos presentados en este 
trabajo muestren diferencias notorias en comparación con las encontradas en estudios previos. Sin realizar un estudio detallado de las propiedades pulsacionales (tema que escapa a los objetivos de esta tesis), puede especularse en base a estos modelos que la energía cinética de oscilación de algunos modos podrían ser considerablemente afectada como resultado de la forma de la frecuencia de Brunt-Väisälä. Remitimos al lector a Córsico, Althaus, Benvenuto \& Serenelli (2001, 2002); Córsico (2002) para un estudio detallado de las propiedades pulsacionales de estos modelos.

Finalmente, cabe mencionar que existen aún muchas incertezas en la teoría de evolución estelar que previenen hacer afirmaciones definitivas acerca de la estratificación química de las enanas blancas. Por ejemplo, el grado en que la semiconvección y el overshooting afectan la estratificación química en el núcleo no es conocido en términos de primeros principios. Por eso, en principio, el perfil de composición química en el núcleo podría ser ligeramente diferente de acuerdo a si estos procesos son tenidos en cuenta o no. Además, la estratificación química en las zonas externas depende del momento en que el progenitor deja la AGB, en particular en cuanto al espesor del buffer de helio. Por último, una pérdida de masa adicional durante la fase de nebulosa planetaria, o durante las primeras etapas en la rama de enfriamiento producirá una disminución adicional del espesor de la envoltura de hidrógeno. 


\section{Capítulo 7}

\section{Enanas blancas de carbono y oxígeno. Progenitor de $7.5 \mathrm{M}_{\odot}$}

\subsection{Introducción}

Desde el punto de vista teórico es sabido que el núcleo de carbono-oxígeno de las WDs desarrolla una fase cristalizada en algún momento de su evolución (Lamb \& Van Horn, 1975). Sin embargo, desde el punto de vista observacional, no ha habido una manera de inferir la existencia de WDs con núcleos cristalizados. En este contexto, el estudio de las estrellas ZZ Ceti parece ser el camino más promisorio para determinar si la cristalización ocurre o no en los interiores de estas estrellas y si ocurre en qué grado lo hace. En efecto, el descubrimiento de pulsaciones en la estrella BPM 37093 (Kannan et al., 1992), una estrella ZZ Ceti masiva que debería estar cristalizada en gran medida (Winget et al., 1997), convierte a esta estrella en un objeto que ofrece excelentes perspectivas para obtener cotas observacionales al proceso de cristalización en las WDs. Montgomery \& Winget (1999) han explorado en detalle desde el punto de vista teórico los efectos de la cristalización en el patrón de los períodos de las pulsaciones y encuentran que algunos períodos son marcadamente afectados por cambios en la fracción de masa cristalizada de sus modelos.

En vista de lo dicho anteriormente, y también en base a que no existen en la literatura modelos de WDs masivas con núcleos de carbono-oxígeno que hayan sido obtenidos teniendo en cuenta su historia evolutiva previa y que además incluyan la difusión de elementos químicos de manera consistente con los cálculos evolutivos (procesos cuya importancia respecto de las propiedades pulsacionales de las estrellas ZZ Ceti ha sido discutida en el capítulo 6 y con más detalle en Córsico, Althaus, Benvenuto \& Serenelli 2001, 2002). Estos modelos permitirán realizar un estudio del espectro pulsacional de las estrellas ZZ Ceti masivas $\left(\sim 1 \mathrm{M}_{\odot}\right)$ que deben encontrarse al menos parcialmente cristalizadas cuando se encuentran en la banda de inestabilidad.

\subsection{Detalles computacionales}

Los cálculos que se presentan en este capítulo han sido realizados con el CEELP cuyas características generales han sido descriptas en el capítulo 2. Para este trabajo sin embargo, se han realizado modificaciones importantes, particularmente en lo que se refiere al tratamiento numérico de los procesos que modifican la composición química en el interior de las estrellas. 
En particular, para el presente estudio se ha considerado un esquema dependiente del tiempo para el tratamiento simultáneo de los cambios de composición química debidos a las reacciones nucleares y a procesos de mezcla macroscópicos (convección, semiconvección e inestabilidades salt-finger conocidas también como Rayleigh-Taylor). Estos cambios pueden describirse mediante el sistema de ecuaciones

$$
\left(\frac{d \vec{Y}}{d t}\right)=\left(\frac{\partial \vec{Y}}{\partial t}\right)_{\mathrm{nuc}}+\frac{\partial}{\partial M_{r}}\left[\left(4 \pi r^{2} \rho\right)^{2} D \frac{\partial \vec{Y}}{\partial M_{r}}\right]
$$

donde el primer término del miembro derecho tiene en cuenta los cambios debidos a las reacciones nucleares y el segundo los cambios por procesos de mezcla. Aquí, $\vec{Y}$ es el vector columna formado por las fracciones número de las especies nucleares consideradas.

Los cambios de abundancias por efectos de las reacciones nucleares están representados por ecuaciones diferenciales no-lineales cuyo tratamiento fue presentado en la sección 2.4 y no lo repetiremos aquí. Baste para nuestros propósitos tener presente que el primer término del miembro derecho de la ecuación 7.1 puede escribirse, una vez hecha la linealización descripta en 2.4, como

$$
\left(\frac{\vec{Y}^{n+1}-\vec{Y}^{n}}{\Delta t}\right)_{\mathrm{nuc}}=-\Gamma \vec{Y}^{n+1}+\vec{\Lambda}
$$

donde $\Gamma$ es una matriz de $I \times I$ (donde $I$ es el número total de especies nucleares consideradas) y $\Lambda$ un vector columna de dimensión $I$ cuyos elementos están dados básicamente por los rates de las reacciones nucleares consideradas, y los supraíndices denotan el paso temporal de la integración (remitirse a la sección 2.4 o a Arnett \& Truran 1969 para más detalles).

Los procesos de mezcla son tratados como procesos difusivos donde el coeficiente de difusión $D$ (igual para todas las especies nucleares consideradas) es calculado a partir de una teoría de convección apropiada (volveremos sobre este punto más adelante). Denotando con subíndices los puntos de la red espacial del modelo estelar, el término difusivo de la ecuación 7.1 puede aproximarse para un tiempo dado $n+1$ como

$$
\left\{\frac{\partial}{\partial M_{r}}\left[\left(4 \pi r^{2} \rho\right)^{2} D \frac{\partial \vec{Y}}{\partial M_{r}}\right]\right\}_{j}^{n+1} \approx \mathcal{A} \vec{Y}_{j-1}^{n+1}+\mathcal{B} \vec{Y}_{j}^{n+1}+\mathcal{C} \vec{Y}_{j+1}^{n+1}
$$

donde $\mathcal{A}, \mathcal{B}$ y $\mathcal{C}$ son matrices múltiplos de la identidad de dimensión $I \times I$ cuyos elementos diagonales están dados por

$$
\begin{aligned}
a_{i i} & =\frac{(4 \pi)^{2}\left(\rho^{2} r^{4} D\right)_{j-1 / 2}}{\left(m_{j-1 / 2}-m_{j+1 / 2}\right)\left(m_{j-1}-m_{j}\right)} \\
c_{i i} & =\frac{(4 \pi)^{2}\left(\rho^{2} r^{4} D\right)_{j+1 / 2}}{\left(m_{j-1 / 2}-m_{j+1 / 2}\right)\left(m_{j}-m_{j+1}\right)} \\
b_{i i} & =-\left(a_{i i}+c_{i i}\right) .
\end{aligned}
$$

donde en todos los casos un $+1 / 2(-1 / 2)$ en el subíndice denota que la cantidad es evaluada en el punto medio entre la capa $j$ y la capa $j+1(j-1)$. Estas definiciones para $\mathcal{A}, \mathcal{B}$ y $\mathcal{C}$ son válidas en todas las capas de la región de la estrella afectada por el proceso de mezcla en cuestión, excepto en los bordes de dicha zona. En estos bordes (p.ej. borde externo de un 
núcleo convectivo, borde interno de una envoltura convectiva, etc.) el flujo de masa debe ser nulo. Por lo tanto tenemos

$$
\frac{\partial}{\partial M_{r}}\left[\left(4 \pi r^{2} \rho\right)^{2} D \frac{\partial \vec{Y}}{\partial M_{r}}\right]_{\text {borde }}=\frac{\partial}{\partial M_{r}}\left[\left(4 \pi r^{2} \rho\right)^{2} D\right] \frac{\partial \vec{Y}}{\partial M_{r}}+\left(4 \pi r^{2} \rho\right)^{2} D \frac{\partial^{2} \vec{Y}}{\partial M_{r}^{2}}
$$

pero el primer término se anula ya que $\partial \vec{Y} / \partial M_{r}=0$ en los bordes de manera que resulta

$$
\frac{\partial}{\partial M_{r}}\left[\left(4 \pi r^{2} \rho\right)^{2} D \frac{\partial \vec{Y}}{\partial M_{r}}\right]_{\text {borde }}=\left(4 \pi r^{2} \rho\right)^{2} D \frac{\partial^{2} \vec{Y}}{\partial M_{r}^{2}}
$$

La derivada segunda puede evaluarse desarrollando en serie de Taylor hasta segundo orden las abundancias (el término de primer orden es nulo), de manera que puede obtenerse finalmente en el borde interno

$$
a_{i i}=\frac{(4 \pi)^{2}\left(\rho^{2} r^{4} D\right)_{1 / 2}}{\left(m_{1}-m_{2}\right)^{2}} ; \quad b_{i i}=-a_{i i} ; \quad c_{i i}=0
$$

y en el borde externo

$$
c_{i i}=\frac{(4 \pi)^{2}\left(\rho^{2} r^{4} D\right)_{J-1 / 2}}{\left(m_{J-1}-m_{J}\right)^{2}} ; \quad b_{i i}=-c_{i i} ; \quad a_{i i}=0
$$

Poniendo juntas las ecuaciones 7.2 y 7.3, el conjunto de ecuaciones diferenciales 7.1 se transforma en el conjunto de ecuaciones lineales

$$
\left[\mathcal{T}^{-1}+\Gamma-\mathcal{B}\right]_{j} \vec{Y}_{j}^{n+1}-\mathcal{A}_{j} \vec{Y}_{j-1}^{n+1}-\mathcal{C}_{j} \vec{Y}_{j+1}^{n+1}=\vec{\Lambda}_{j}+\frac{\vec{Y}_{j}^{n}}{\Delta t}
$$

donde $\mathcal{T}^{-1}$ es una matriz múltiplo de la identidad, también de dimensiones $I \times I$, cuyos elementos diagonales valen $1 / \Delta t$.

Definiendo $\mathcal{F}_{j}=\mathcal{T}_{j}^{-1}+\Gamma_{j}-\mathcal{B}_{j}$ y $\overrightarrow{\mathcal{M}}_{j}=\vec{\Lambda}_{j}+\vec{Y}_{j}^{n} / \Delta t$ para cada capa del modelo, entonces el sistema de ecuaciones resultante puede representarse esquemáticamente como

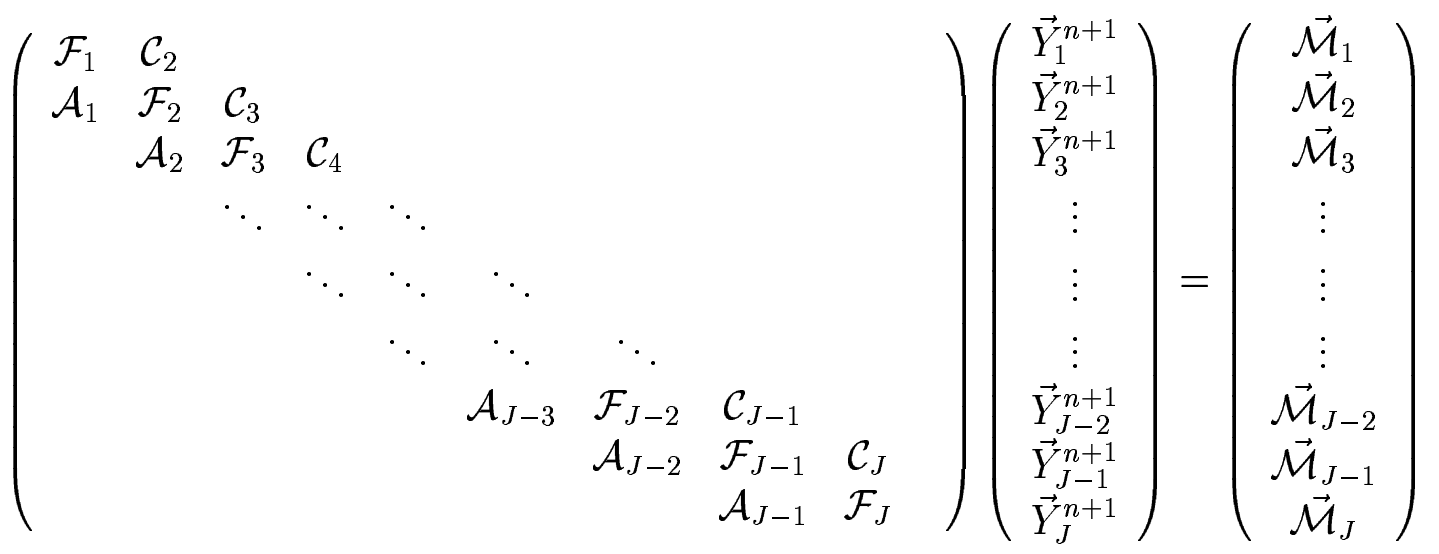

donde los subíndices denotan la capa del modelo. La matriz tiene elementos nulos fuera de la banda mostrada.

En el caso presente, se han incluido 16 especies nucleares y los modelos tienen durante la etapa de los pulsos térmicos unas 1500 capas, por lo que el sistema de ecuaciones puede tener (dependiendo de la extensión de las regiones afectadas por la mezcla) una dimensión 
aproximada de $(16 \times 1500) \times(16 \times 1500)$. Para resolver el sistema de ecuaciones empleamos el método presentado en Iben \& MacDonald (1985), que hace uso de la forma particular (tipo tridiagonal) del sistema, y referimos al lector a ese trabajo para más detalles.

Las especies nucleares consideradas en los cálculos aquí presentados son $\mathrm{H}, \mathrm{D},{ }^{3} \mathrm{He},{ }^{4} \mathrm{He}$, ${ }^{7} \mathrm{Li},{ }^{7} \mathrm{Be},{ }^{12} \mathrm{C},{ }^{13} \mathrm{C},{ }^{14} \mathrm{~N},{ }^{15} \mathrm{~N},{ }^{16} \mathrm{O},{ }^{17} \mathrm{O},{ }^{18} \mathrm{O},{ }^{19} \mathrm{~F},{ }^{20} \mathrm{Ne}$ y ${ }^{22} \mathrm{Ne}$ ligadas entre sí por 34 reacciones nucleares hasta la quema del carbono. Las reacciones nucleares están tomadas de Caughlan \& Fowler (1988) y también de Angulo C. et al. (1999).

Como se mencionó al comienzo de esta sección, la convección no es el único mecanismo de mezcla que puede ocurrir en los interiores estelares. En efecto, otros procesos tales como la semiconvección y la inestabilidad salt-finger también pueden tener lugar durante la evolución de las estrellas. Para tener en cuenta estos procesos por medio de la ecuación 7.1, el coeficiente de difusión $D$ debe ser calculado para cada uno de los procesos considerados a partir de una teoría de convección dada. En el presente trabajo se ha incorporado en el CEELP la teoría de convección extendida de la longitud de mezcla para fluidos con gradientes de composición dada por Grossman, Narayan \& Arnett (1993) en su aproximación local (Grossman \& Taam, 1996). Según este formalismo, en una región de la estrella donde tiene lugar alguno de los procesos de mezcla mencionados, el coeficiente de difusión $D$ está dado por

$$
D=\ell \sigma
$$

donde $\ell$ es la longitud media de mezcla y $\sigma$ la velocidad turbulenta del medio. El valor de $\sigma$ se determina resolviendo simultáneamente las ecuaciones para la velocidad turbulenta y la ecuación de la conservación del flujo de energía (ecuaciones 9 y 17 de Grossman \& Taam 1996). La resolución de estas ecuaciones determina también el valor del gradiente de temperatura.

Un punto importante a mencionar es que, como se dijo en la Introducción, estos cálculos incluyen durante la etapa de enana blanca la difusión microscópica de elementos. La implementación de estos procesos en el CEELP ha sido descripta en 3.2 y se incluyen la sedimentación gravitatoria y las difusiones química y térmica para ${ }^{1} \mathrm{H},{ }^{3} \mathrm{He},{ }^{4} \mathrm{He},{ }^{12} \mathrm{C},{ }^{14} \mathrm{~N}$ y ${ }^{16} \mathrm{O}$.

El objetivo principal de este trabajo es obtener modelos de WDs con núcleos de carbonooxígeno masivas, apropiados para el estudio pulsacionales de estas estrellas. El progenitor de WD es el resultado de la evolución de estrellas de masas intermedias (en el presente trabajo la masa inicial del modelo es de $7.5 \mathrm{M}_{\odot}$ ) que sufren episodios de pérdida de masa a lo largo de su evolución, particularmente durante su evolución en la AGB. Seguir en detalle la evolución del progenitor de estas WDs es importante ya que la estructura inicial de la WD, cuando ésta entra en la rama de enfriamiento, y en particular los perfiles químicos, está determinada por la evolución previa y en especial durante la etapa de los pulsos térmicos que ocurren en el extremo de la AGB. Siguiendo la formulación dada por Blöcker (1995a) (basada en cálculos dinámicos para atmósferas de estrellas tipo Mira), la tasa de pérdida de masa está dada por la fórmula de Reimers

$$
\dot{M}=4 \times 10^{-13} \eta_{R} \frac{L R}{M} ; \quad\left[M_{\odot} / \mathrm{yr}\right]
$$

a lo largo de la RGB y al comienzo de la evolución a lo largo de la AGB. $\eta_{R}$ es un parámetro que se ha considerado igual a 1 en este trabajo, $M$ es la masa de la estrella, $R$ su radio y $L$ su luminosidad. Cuando el período $P_{0}$ del modo fundamental de oscilación dado por (Ostlie \& Cox, 1986)

$$
\log \left(P_{0} / d\right)=-1.92-0.73 \log M+1.86 \log R
$$




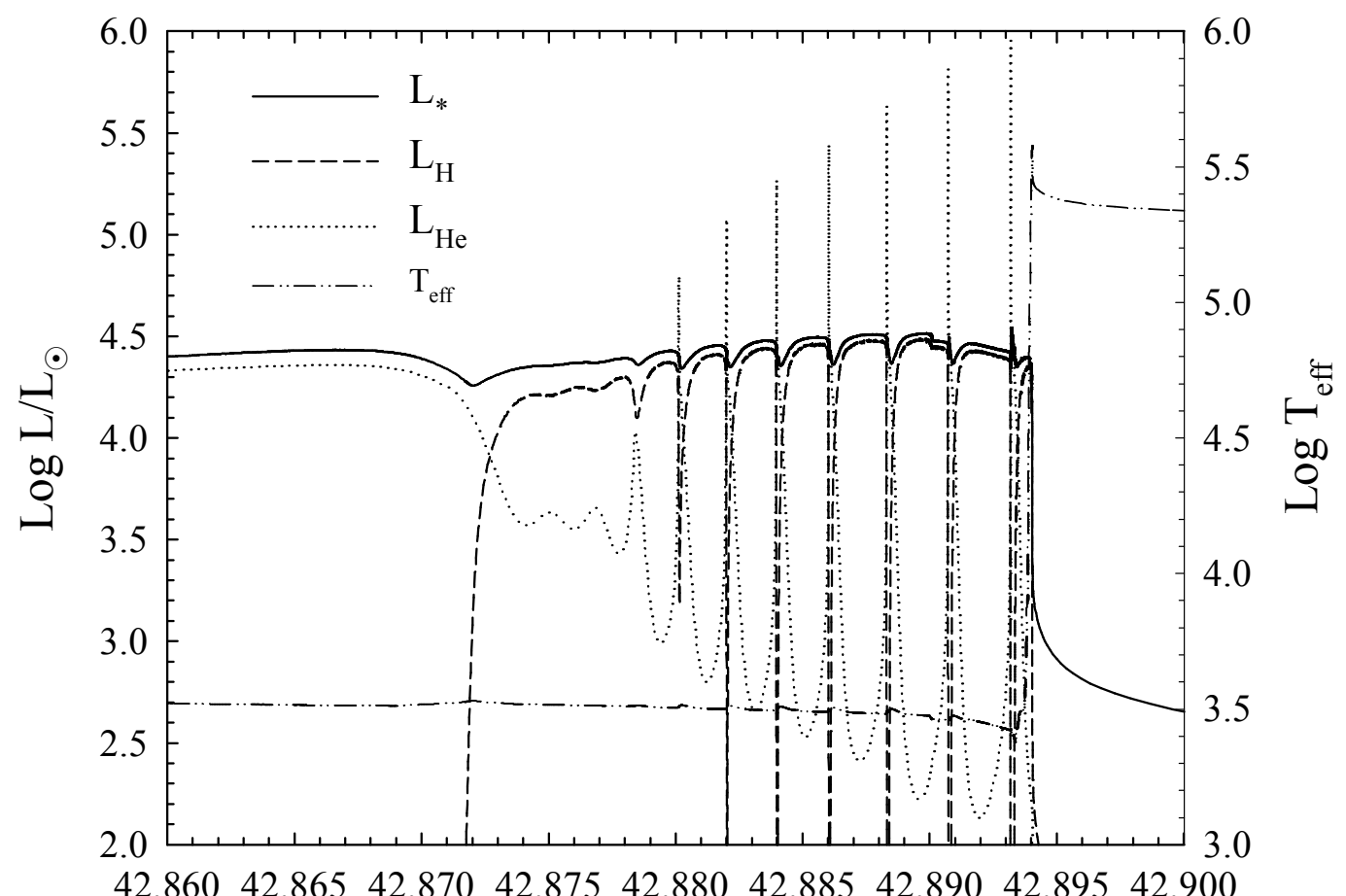

Age $\left[10^{6}\right.$ years $]$

Figura 7.1: Evolución de la luminosidad superficial $L_{*}$, luminosidad por combustión del hidrógeno $L_{H}$ (cadenas protón-protón + ciclo CNO), luminosidad por combustión del helio $L_{H e}$ (todas en unidades solares) y temperatura efectiva ( $\mathrm{T}_{\text {eff }}$ ) durante la fase que va desde un poco antes de la ocurrencia de los pulsos térmicos hasta que comienza la evolución a lo largo de la rama de enfriamiento de la WD remanente de $0.936 \mathrm{M}_{\odot}$. Notar que la estrella deja la AGB (momento que corresponde al aumento de la $\mathrm{T}_{\text {eff }}$ ) cuando la combustión del hidrógeno es la fuente dominante de energía de la estrella.

es mayor que 100d la tasa de pérdida de masa está dada por

$$
\dot{M}=4.83 \times 10^{-9} M^{-2.1} L^{2.7} \dot{M}_{R} ; \quad\left[M_{\odot} / \mathrm{yr}\right],
$$

donde $\dot{M}_{R}$ es la tasa de pérdida de masa de Reimers dada por la ecuación 7.14.

\subsection{Resultados y discusión}

En este trabajo se ha considerado la evolución de una estrella de $7.5 \mathrm{M}_{\odot}$, con metalicidad $Z=0.02$, comenzando desde la ZAMS y durante las etapas de combustión del hidrógeno y del helio en el núcleo hasta el extremo de la AGB durante la etapa de los pulsos térmicos. Posteriormente se sigue la evolución de la WD remanente hasta que ésta alcanza la franja de inestabilidad en el dominio de las estrellas ZZ Ceti.

La evolución de estrellas de masas intermedias ha sido estudiada previamente por diversos investigadores (Blöcker, 1995a,b; Domínguez et al., 1999) y referimos al lector a esos trabajos para detalles de la misma. Dado que el objetivo principal de este trabajo es obtener modelos detallados de estrellas ZZ Ceti masivas, nos limitamos aquí a describir brevemente los resultados 


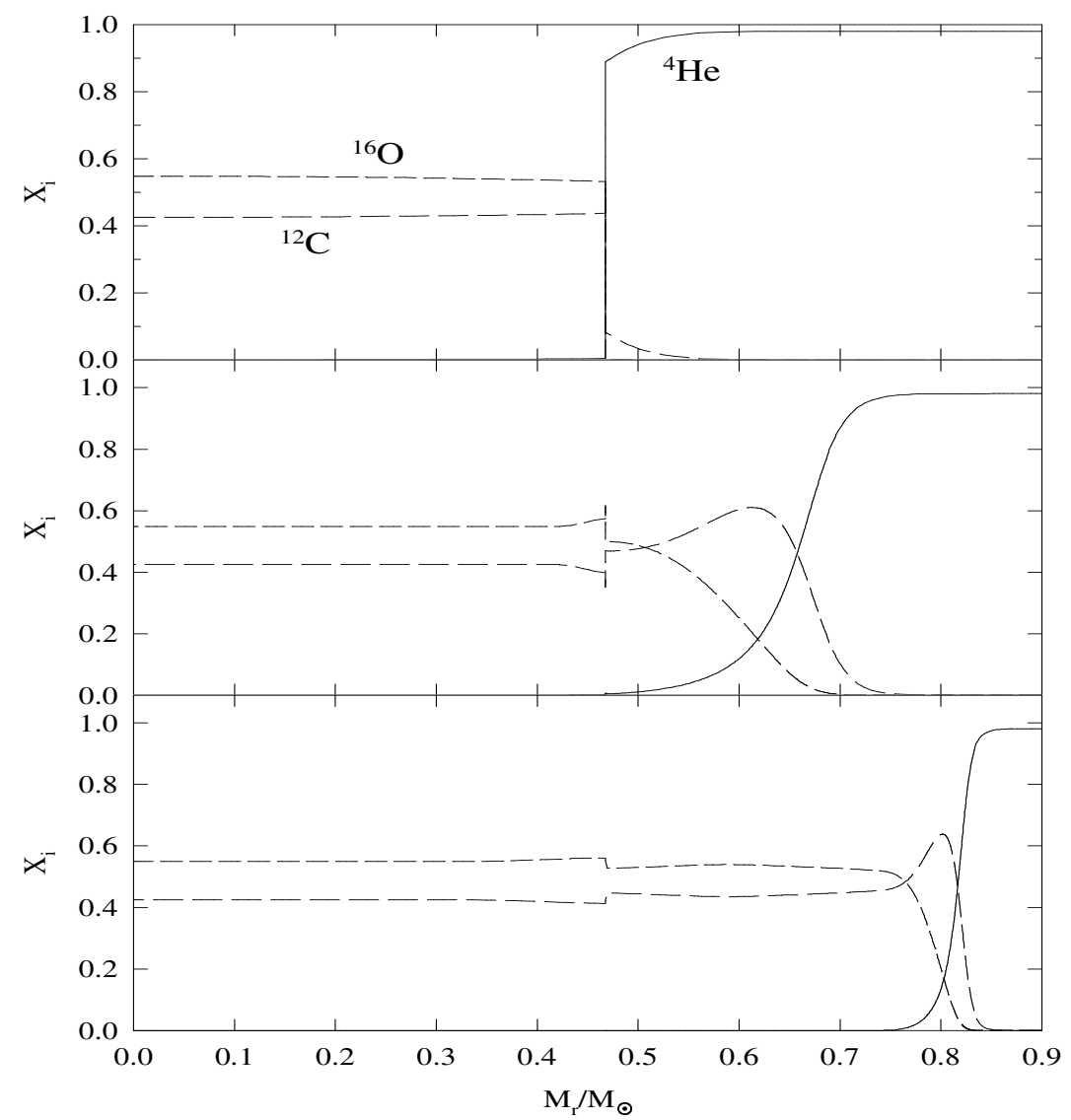

Figura 7.2: Perfiles internos de las abundancias de ${ }^{4} \mathrm{He},{ }^{12} \mathrm{C}$ y ${ }^{16} \mathrm{O}$ para el modelo de $7.5 \mathrm{M}_{\odot}$ en tres etapas evolutivas después de la fase de combustión de helio en el núcleo. Se muestran sólo las $0.9 \mathrm{M}_{\odot}$ internas del modelo (las partes externas forman la envoltura convectiva y por lo tanto las abundancias de cada especie nuclear son constantes en esa región). En $M_{r} \approx 0.45 \mathrm{M}_{\odot}$ se desarrolla una inestabilidad salt-finger por efectos del gradiente negativo en el peso molecular (panel medio) que lleva a una redistribución de las abundancias del ${ }^{12} \mathrm{C}$ y del ${ }^{16} \mathrm{O}$ en las regiones más internas del modelo (panel inferior).

de nuestros cálculos correspondientes a las etapas previas que llevan a la formación de estos objetos.

El modelo de 7.5 $\mathrm{M}_{\odot}$ evoluciona desde la ZAMS hasta la RGB en unos 36 Myr. Cuando el modelo comienza a desarrollar una envoltura convectiva y comienza a ascender por la RGB comienza a aplicarse la pérdida de masa siguiendo el formulismo que especificado en la sección previa. La etapa de combustión del helio nuclear dura $5.8 \times 10^{6}$ años y posteriormente a esta etapa la estrella comienza a ascender por la AGB. A diferencia de los resultados correspondiente al modelo de $3 \mathrm{M}_{\odot}$ presentados en el capítulo 6, al finalizar la etapa de combustión del helio nuclear no se producen los micropulsos térmicos.

Cuando el modelo asciende por la AGB y alcanza por primera vez una luminosidad superficial de $\log L / \mathrm{L}_{\odot} \approx 4.45$ comienza la etapa de los pulsos térmicos. La evolución durante esta etapa de la luminosidad superficial $L_{*}$, luminosidad por combustión del hidrógeno $L_{H}$, luminosidad por combustión del helio $L_{H e}$ y temperatura efectiva $\left(\mathrm{T}_{\text {eff }}\right)$ se muestran en la figura 7.1. El período interpulso es de unos $2-2.2 \times 10^{3}$ años y durante esta etapa la masa 

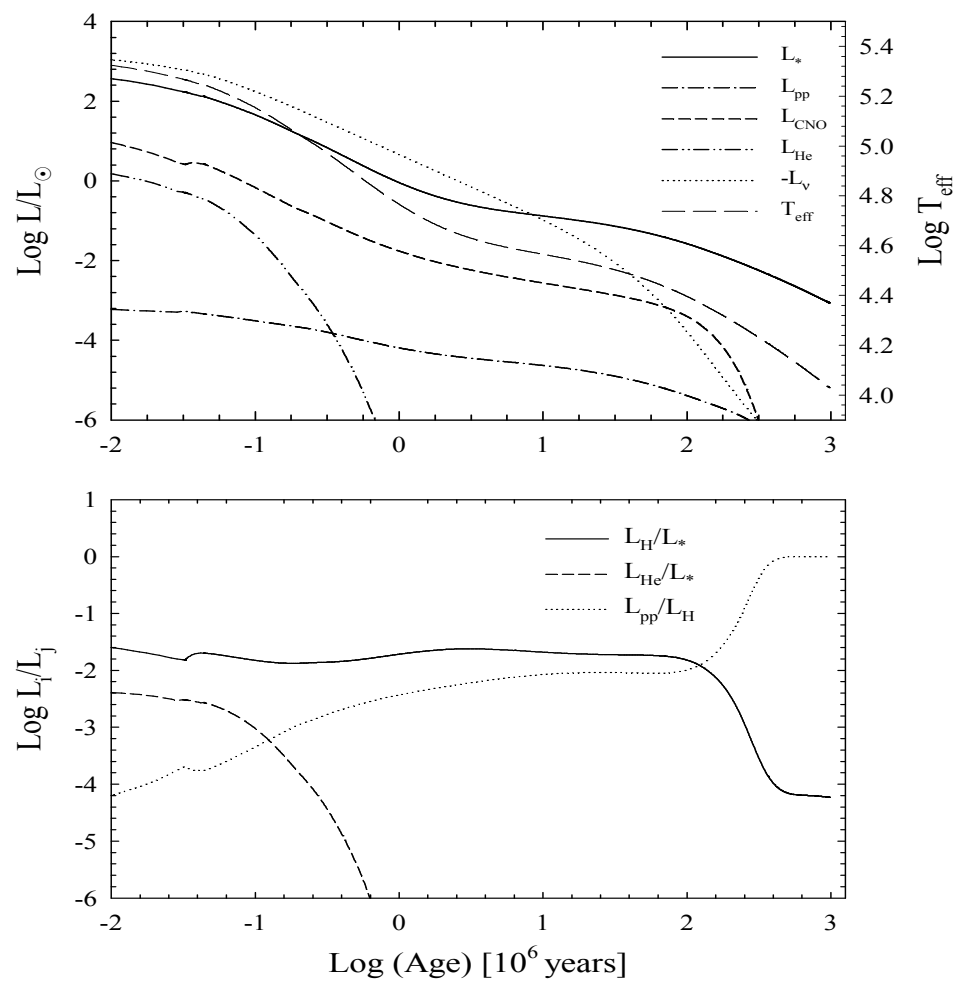

Figura 7.3: El panel superior muestra la evolución en función del tiempo de $L_{*}, L_{p p}, L_{C N O}, L_{H e}$ y $L_{\nu}$ (todas en unidades solares) durante la etapa de WD del remanente de $0.936 \mathrm{M}_{\odot}$. También se muestra la temperatura efectiva $\mathrm{T}_{\text {eff }}$. Aquí el punto de edad cero corresponde al momento en que la pérdida de masa es suprimida. El panel inferior muestra los cocientes de luminosidades. $L_{H}$ es la luminosidad total por combustión de hidrógeno $\left(L_{H}=L_{p p}+L_{C N O}\right)$. La liberación de energía nuclear es prácticamente despreciable como fuente de energía de la WD durante toda su evolución, siendo esto particularmente notable durante la etapa de ZZ Ceti donde apenas contribuye con un $0.01 \%$ a la luminosidad de la estrella.

de la estrella se reduce desde unas $6.7 \mathrm{M}_{\odot}$ (la pérdida de masa en las etapas previas es bastante reducida) hasta el valor final del remanente, que es de $0.936 \mathrm{M}_{\odot}$. Puede verse en esta figura que a medida que el modelo pierde masa evoluciona hacia $\mathrm{T}_{\text {eff }}$ más bajas y radios más grandes (en términos generales, tener en cuenta las oscilaciones producidas por lo pulsos) lo que se traduce en un aumento de la tasa de pérdida de masa, que alcanza valores del orden de $10^{-3.7} \mathrm{M}_{\odot} /$ yr. Después del $7 \mathrm{mo}$. pulso la envoltura rica en hidrógeno se vuelve demasiado delgada como para permanecer extendida. La zona convectiva externa desaparece y la estrella evoluciona apartándose de la AGB. Cuando la estrella alcanza una $\mathrm{T}_{\text {eff }}$ de $8000 \mathrm{~K}$ suprimimos la pérdida de masa, en ese momento la masa de la envoltura de hidrógeno es de $5.1 \times 10^{-5} \mathrm{M}_{\odot}$. En este caso, el apartamiento de la AGB ocurre cuando la combustión del shell de hidrógeno es la fuente principal de energía de la estrella. Consecuentemente no se observa un retorno de la estrella a la AGB, sino que ésta evoluciona directamente hacia el dominio de las WDs. Al igual que en los resultados presentados en el capítulo 6, no se han invocado episodios de pérdida de masa durante la etapa de núcleo de nebulosa planetaria o etapas calientes en la rama de enfriamiento. De esta manera, el valor de la masa de la envoltura de hidrógeno obtenido en estos cálculos debe considerarse como un límite superior.

En la figura 7.2 se muestra la evolución de la distribución interna de ${ }^{4} \mathrm{He},{ }^{12} \mathrm{C}$ y ${ }^{16} \mathrm{O}$ como 


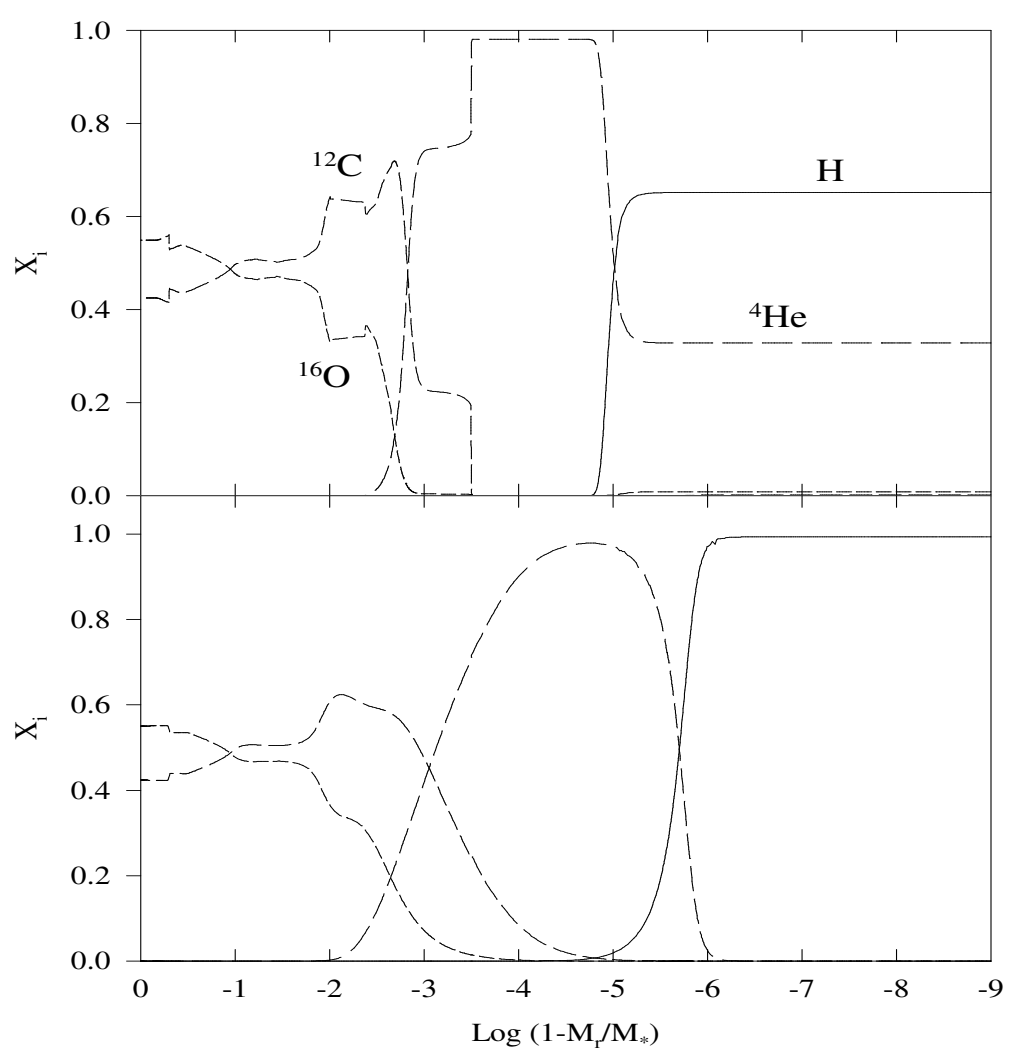

Figura 7.4: Abundancia por masa de ${ }^{1} \mathrm{H},{ }^{4} \mathrm{He},{ }^{12} \mathrm{C}$ y ${ }^{16} \mathrm{O}$ como función de la fracción de masa externa para la WD remanente de $0.936 \mathrm{M}_{\odot}$ al comienzo de la rama de enfriamiento (panel superior) y cerca de la banda de inestabilidad de las ZZ Ceti (panel inferior). Los modelos están caracterizados por valores $\left(\log \left(L / \mathrm{L}_{\odot}\right), \log \mathrm{T}_{\text {eff }}\right)$ de $(2.24,5.27)$ y $(-2.92,4.06)$ (paneles superior e inferior respectivamente). Claramente, la difusión de elementos modifica notablemente los perfiles químicos.

función de la masa durante etapas posteriores a la combustión del helio en el núcleo de la estrella. El panel superior muestra los perfiles químicos después de $4.19 \times 10^{7}$ años de evolución, cuando el núcleo convectivo desaparece, dejando una abundancia por masa de ${ }^{16} \mathrm{O}$ de 0.55 en el centro. A medida que la evolución continúa, las capas internas de ${ }^{4} \mathrm{He}$ que rodean la zona que formó previamente el núcleo convectivo de helio son quemadas de manera radiativa dando lugar a un pico en las abudancias de ${ }^{12} \mathrm{C}$ y ${ }^{16} \mathrm{O}$ ubicado en $M_{r} \approx 0.45 \mathrm{M}_{\odot}$. Esto se muestra en el panel medio de la figura 7.2, que corresponde al momento en que el modelo alcanza por primera vez $\log \left(L / l_{\odot}\right)=3.72$ después de $4.25 \times 10^{7}$ años de evolución. En la zona donde el pico se forma, el peso molecular decrece hacia el interior de la estrella, lo que da origen a una estabilidad de tipo salt-finger en el pico de las abundancias de ${ }^{12} \mathrm{C}$ y ${ }^{16} \mathrm{O}$. La mezcla inducida por esta inestabilidad es responsable de la resdistribución de la parte más interna del perfil de ${ }^{12} \mathrm{C}$ y ${ }^{16} \mathrm{O}$ que puede observarse en el panel inferior de la misma figura. Esta redistribución ocurre durante $\operatorname{los} 3 \times 10^{5}$ años subsiguientes al desarrollo de la inestabilidad. En el mismo panel puede verse también que la masa del núcleo de ${ }^{12} \mathrm{C} /{ }^{16} \mathrm{O}$ se ha incrementado notablemente por efectos de la combustión del shell de helio.

En la figura 7.3 se muestra la evolución en función del tiempo a lo largo de la rama de enfriamiento de las luminosidaded superficial, por combustión del hidrógeno vía cadena protón- 
protón $L_{p p}$, por combustión del hidrógeno vía ciclo CNO $L_{C N O}$, por combustión del helio $L_{H e}$ y por pérdidas por neutrinos $L_{\nu}$ y se incluye además la evolución de la $\mathrm{T}_{\text {eff. }}$ El punto cero para el tiempo corresponde al momento en que se suprimió la pérdida de masa. Durante las primeras etapas evolutivas el enfriamiento de la estrella está dominado por la emisión de neutrinos (debido a la alta temperatura del núcleo degenerado de la WD). La liberación de energía nuclear, dominada hasta unos $300 \mathrm{Myr}$ de evolución por el ciclo CNO, es una fuente mínima de energía para la estrella, contribuyendo con alrededor de un $3 \%$ a la energía total radiada por la estrella. Es interesante destacar, sin embargo, que aún cuando la energía nuclear represente una contribución menor a la energía total de que dispone la estrella, si la difusión de elementos no es considerada esto resulta aún más dramático. En efecto, en este último caso la liberación de energía nuclear se vuelve completamente despreciable en sólo menos de $1 \mathrm{Myr}$ (ver Blöcker 1995b para un resultado similar). La diferencia es consecuencia de los efectos de la difusión química que alimenta las capas interiores de la envoltura de la estrella, más calientes, con hidrógeno que se difunde lentamente hacia el interior, manteniendo encendidas las reacciones nucleares por mucho más tiempo que en el caso en que la difusión no es tenida en cuenta. Después de los $300 \mathrm{Myr}$ iniciales el ciclo CNO se apaga totalmente y sólo persiste una liberación de energía nuclear marginal a través de las cadenas protón-protón (panel inferior de la figura 7.3) debido a su menor dependencia con la temperatura. Durante la fase de ZZ Ceti la liberación de energía nuclear es totalmente despreciable.

Una vez que la WD remanente ha alcanzado la rama de enfriamiento, la distribución de la composición química es modificada por efectos de los procesos de difusión. Los cambios producidos en estos perfiles pueden verse en la figura 7.4, en la cual se comparan los perfiles químicos al comienzo de la rama de enfriamiento (panel superior) y durante la etapa de ZZ Ceti (panel inferior). El perfil químico emergente de la fase de los pulsos térmicos durante la evolución en la $\mathrm{AGB}$, está caracterizado por una zona rica tanto en ${ }^{4} \mathrm{He}$ como en ${ }^{12} \mathrm{C}$ ubicada en $\log \left(1-M_{r} / \mathrm{M}_{\odot}\right) \approx-3.25$, y por encima una zona casi pura de helio (llamada buffer de helio, ya mencionada en el capítulo 6 ) de $\approx 2.5 \times 10^{-4} \mathrm{M}_{\odot}$. La masa de hidrógeno que queda en la envoltura de la estrella al comienzo de la rama de enfriamiento es de $\approx 6.2 \times 10^{-6} \mathrm{M}_{\odot}$. Durante el enfriamiento de la WD, la distribución química es profundamente alterada por la difusión de elementos. Los perfiles químicos originados en la AGB son prácticamente borrados por los procesos de difusión, como puede verse mediante una comparación entre ambos paneles de la figura 7.4. En particular, las variaciones muy abruptas de los perfiles al comienzo de la evolución del remanente como WD (casi discontinuos en las zonas que marcan los límites de las regiones convectivas durante la evolución previa) son fuertemente suavizadas por efectos de la difusión cuando la estrella alcanza la banda de inestabilidad de las ZZ Ceti. Dada la importancia primaria de la estratificación química de la estrella en sus propiedades pulsacionales, los cambios inducidos por la difusión tendrán efectos notorios sobre el espectro pulsacional de estos modelos (ver el capítulo 6 y Córsico, Althaus, Benvenuto \& Serenelli 2001, 2002 para el caso de una WD de $0.563 \mathrm{M}_{\odot}$ ). Notar también que en el modelo existe una interface química que está caracterizada por abundancias apreciables de ${ }^{4} \mathrm{He},{ }^{12} \mathrm{C}$ y ${ }^{16} \mathrm{O}$. Cuando la WD alcanza la etapa de ZZ Ceti, esta interface tiene una extensión considerable por efectos de la difusión. Con respecto a la envoltura de hidrógeno, ha sido reducida hasta $2.3 \times 10^{-6} \mathrm{M}_{\odot}$ por efectos de la combustión residual de hidrógeno cuando la WD alcanza el dominio de las ZZ Ceti.

Finalmente, en relación a la cristalización del interior de la WD, cabe mencionar que nuestro modelo comienza a cristalizarse cuando éste entra en la franja de inestabilidad de las ZZ Ceti, y cuando alcanza el borde rojo de la misma la masa cristalizada abarca aproximadamente un $25 \%$ de la masa estelar. Dado que no estamos interesados en estudiar las escalas temporales 


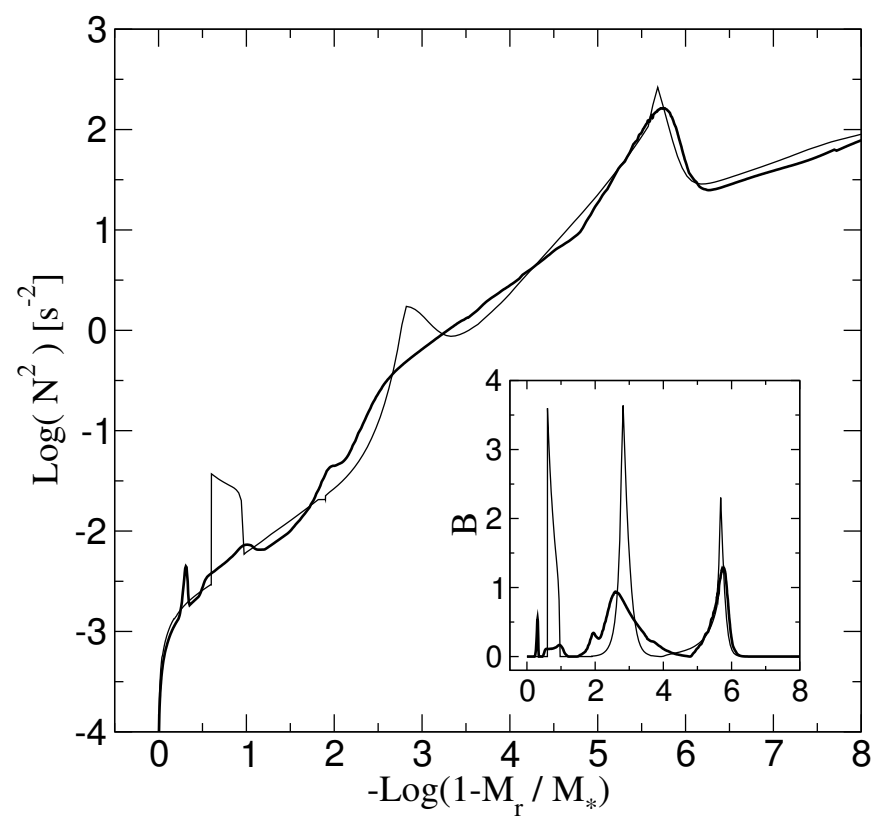

Figura 7.5: Logaritmo del cuadrado de la frecuencia de Brunt-Väisälä y el término de Ledoux $B$ predichos por nuestros modelos. Los resultados de los modelos de Montgomery \& Winget (1999) se muestran en líneas delgadas.

de enfriamiento, no hemos incluido la liberación del calor latente como término adicional de energía.

Al igual que en el caso presentado en el capítulo 6, analizamos las propiedades de la frecuencia de Brunt-Väisälä para el momento en que nuestro modelo entra en la banda de inestabilidad de las WD DA, o ZZ Ceti. Para esto consideremos un modelo correspondiente a una $\mathrm{T}_{\text {eff }} \approx 12000 \mathrm{~K}$. Como es bien sabido, la distribución de abundancias químicas produce características distintivas en la frecuencia de Brunt-Väisälä $N$ que son responsables, en última instancia, de las propiedades pulsacionales de la estrella. En este sentido, se muestra en la figura $7.5 \mathrm{~N}^{2}$ y también el término de Ledoux $B$ (ver ecuaciones 6.2 y 6.3 para una definición de ambas cantidades). También se incluyen en la misma figura las predicciones de los modelos estelares considerados en Montgomery \& Winget $(1999)^{1}$ (en líneas delgadas). Notar que los perfiles de $B$ y $N^{2}$ dados por nuestros modelos difieren apreciablemente de los dados por Montgomery \& Winget (1999). Este comportamiento diferente puede entenderse en parte en base a que estos autores invocan el equilibrio difusivo en la aproximación de elemento-traza para determinar la forma de los perfiles químicos en las zonas de transición de abundancias. Por el contrario, nuestro tratamiento de la difusión predice perfiles químicos suaves lo que lleva a que el comportamiento de los perfiles de $B$ y $N$ no presente características tan marcadas. Como ya se ha mencionado, nuestros modelos predicen la existencia de una región donde ${ }^{4} \mathrm{He},{ }^{12} \mathrm{C}$ y ${ }^{16} \mathrm{O}$ resultan abundantes. Esta zona de transición da dos contribuciones a $B$, una de ellas está localizada en $\log \left(1-M_{r} / \mathrm{M}_{*}\right) \approx-2.5$ y la otra de amplitud menor en $\log \left(1-M_{r} / \mathrm{M}_{*}\right) \approx-2$. Por último es importante notar que la estructura presente en $B$ en las zonas más internas de la estrella es menos pronunciada que en los modelos de Montgomery \& Winget (1999). Este efecto es por supuesto debido a la diferente distribución de ${ }^{12} \mathrm{C} /{ }^{16} \mathrm{O}$ en el núcleo de la WD.

\footnotetext{
${ }^{1}$ La comparación está realizada con un modelo de $0.94 \mathrm{M}_{\odot}$ y $\mathrm{T}_{\text {eff }}=12000 \mathrm{~K}$ cedido por el Dr. M. Montgomery
} 


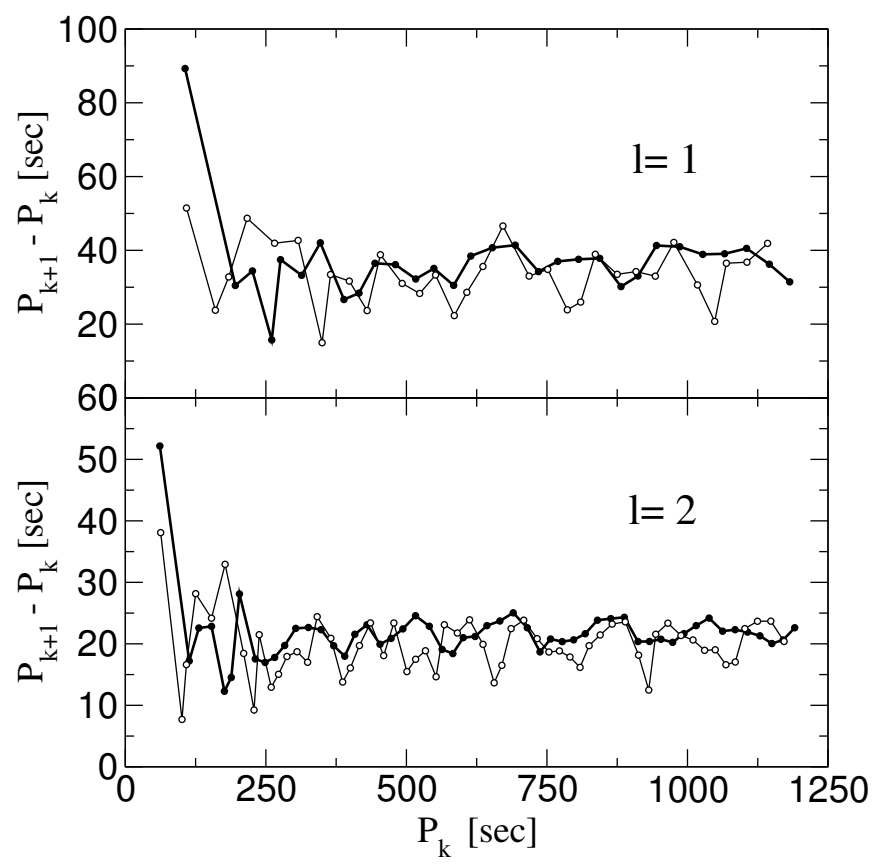

Figura 7.6: Espaciamiento de períodos para $\ell=1$ (panel superior) y $\ell=2$ (panel inferior) en términos de los períodos calculados $P_{k}$. Los puntos llenos y líneas gruesas corresponden a nuestro modelo. Los puntos vacíos y líneas delgadas corresponden a los modelos de Montgomery \& Winget (1999).

Un estudio detallado de las propiedades pulsacionales de estos modelos está más allá de los alcances de esta tesis. Un análisis detallado de las mismas puede encontrarse en Córsico (2002) y nos limitamos aquí a una presentación breve, comentando las implicancias más relevantes que se derivan del mismo. El espectro pulsacional del nuestro modelo fue calculado usando el código de pulsaciones descripto en Córsico, Althaus, Benvenuto \& Serenelli (2001). Específicamente se han calculado los modos-g con $\ell=1,2$ y 3 para el rango de períodos $\left(P_{k}\right.$, siendo $k$ el orden radial del modo) esperados para las estrellas ZZ Ceti. Hay que mencionar que si bien nuestro modelo se halla parcialmente cristalizado en la banda de inestabilidad, los cálculos pulsacionales se han realizado asumiendo que toda la estrella es fluida. La figura 7.6 muestra el espaciamiento de períodos $\Delta P_{k}=P_{k+1}-P_{k}$ para $\ell=1 \mathrm{y} 2$. Las líneas gruesas y puntos llenos muestran los resultados obtenidos con nuestro modelo, mientras que las líneas delgadas y puntos vacíos muestran los correspondientes al modelo de Montgomery \& Winget (1999). El aspecto más importante que puede observarse en la figura 7.6 es que el fenómeno de los modos atrapados predicho por nuestro modelo resulta mucho menos notorio, excepto en el caso de los períodos más cortos, en comparación a los resultados de Montgomery \& Winget (1999). Creemos que esta diferencia es atribuible a la forma mucho más suave de $N^{2}$ que caracteriza a nuestros modelos. El comportamiento más suave de $\Delta P_{k}$ es un resultado no sólo del empleo de perfiles químicos cuya forma evoluciona con el tiempo por efectos de la difusión (efecto particularmente notable en las capas externas, ver Córsico, Althaus, Benvenuto \& Serenelli 2001), sino también debido a la composición química del núcleo que se originó durante la evolución del progenitor de WD. Hay que mencionar también que los valores de $\Delta P_{k}$ obtenidos son, en promedio, ligeramente superiores a los hallados por Montgomery \& Winget (1999), hecho que se debe a la densidad central menor que caracteriza a nuestro modelo. 


\subsection{Conclusiones}

Las WDs con núcleos de carbono-oxígeno cubren un rango de masas entre $\sim 0.5$ y $\sim 1.1 \mathrm{M}_{\odot}$ aproximadamente $^{2}$. En el caso de las WD cerca del límite superior de este rango, se espera que durante su evolución a través de la franja de inestabilidad de las ZZ Ceti, sus núcleos se hallen parcialmente cristalizados; y esto debe manifestarse en las propiedades pulsacionales de las estrellas ZZ Ceti masivas.

Para obtener modelos apropiados para estudiar las pulsaciones de este tipo de objetos, se ha realizado un cálculo evolutivo de una estrella de $7.5 \mathrm{M}_{\odot}$ partiendo desde la ZAMS y a lo largo de las etapas de combustión de hidrógeno y helio en el núcleo, y posteriormente a lo largo de la AGB y finalmente durante la etapa de los pulsos térmicos en el extremo de la misma. Por efectos de la pérdida de masa aplicada, la estrella deja la AGB después de 7 pulsos térmicos, evolucionando rápidamente hacia la configuración de enana blanca. El remanente tiene $0.936 \mathrm{M}_{\odot}$ y su enfriamiento está dominado inicialmente por la emisión de neutrinos (mientras su temperatura central es mayor a $50 \times 10^{7} \mathrm{~K}$ ). La liberación de energía nuclear representa una mínima contribución a la energía de que dispone la estrella y en particular durante la etapa de ZZ Ceti es completamente despreciable.

Los perfiles químicos iniciales de la WD remanente corresponden a aquellos resultantes de la evolución de su progenitor. Las abundancias centrales por masa son $X_{{ }^{12} \mathrm{C}}=0.425, X_{16} \mathrm{O}=$ $0.55, X_{22} \mathrm{Ne}=0.019$ y trazas de las demás especies nucleares. Las interfaces están determinadas principalmente por el último episodio de mezcla convectiva ocurrido durante los pulsos térmicos y son prácticamente discontinuas. La masa de la envoltura de hidrógeno es, al comienzo de la rama de enfriamiento, $6.2 \times 10^{-6} \mathrm{M}_{\odot}$. A lo largo de la evolución de la rama de enfriamiento, la difusión de elementos modifica notablemente los perfiles químicos iniciales, y las casi discontinuidades observadas al comienzo son suavizadas de forma notoria. En el momento de alcanzar la fase de ZZ Ceti, la envoltura de hidrógeno ha sido reducida a $2.3 \times 10^{-6} \mathrm{M}_{\odot}$ por efectos de la combustión nuclear residual.

Una comparación con las predicciones hechas por modelos que incorporan el equilibrio difusivo, en la aproximación elemento traza, muestra que los efectos de la difusión tienen importantes consecuencias en la forma del término de Ledoux y, en consecuencia, en la frecuencia de Brunt-Väisälä, que presentan un comportamiento mucho más suave en las zonas de transición de composición química. Es importante destacar, así mismo, que existe una región de la estrella caracterizado por una composición donde el ${ }^{4} \mathrm{He}$, el ${ }^{12} \mathrm{C}$ y el ${ }^{16} \mathrm{O}$ son comparables en abundancia, por lo que la aproximación de elemento traza resulta inaplicable para determinar la forma de los perfiles químicos. Como ha sido mostrado en el caso de un modelo de $0.563 \mathrm{M}_{\odot}$ (Córsico, Althaus, Benvenuto \& Serenelli, 2001, 2002), es razonable suponer que las diferencias en la forma del perfil de la frecuencia de Brunt-Väisälä producidos por la inclusión de la difusión de elementos tendrán efectos notorios en el espectro pulsacional de estos objetos, en particular en lo que respecta a los llamados modos atrapados. En efecto, una primera aplicación al estudio del espectro pulsacional realizada con nuestro modelo revela que el efecto de modo atrapados es mucho menos notorio que en el caso en que el equilibrio difusivo es empleado para modelar las interfaces químicas. Este comportamiento más suave en la distribución de los períodos de los modos permite esperar que la señal de la cristalización en el espectro pulsacional de las estrellas ZZ Ceti masivas no quede enmascarada por el "ruido" que introducen los perfiles químicos. Para más detalles referirse a Córsico (2002).

\footnotetext{
${ }^{2}$ Algunos autores, sin embargo, sugieren posibles escenarios donde estas estrellas podrían formarse con tan bajas como $0.35 \mathrm{M}_{\odot}$.
} 


\section{Conclusiones}

El trabajo presentado en esta tesis tiene un tronco común, la evolución de estrellas enanas blancas, pero puede dividirse en dos ramas bien diferenciadas tanto por el tipo de objetos estudiados como por los objetivos particulares buscados en cada caso.

La primera de estas ramas es la que corresponde al estudio de las enanas blancas de baja masa, con núcleos de helio (HeWDs). En el caso de estas estrellas el énfasis estuvo puesto en la obtención de modelos evolutivos que cubrieran todo el rango de luminosidades en que estos objetos son (o pueden) ser observados y se buscó además cubrir el rango de masas abarcado por estas estrellas. Los principales resultados se enumeran a continuación.

La evolución binaria que lleva a la formación de las HeWDs predice que estas estrellas son formadas con envolturas de hidrógeno suficientemente gruesas como para soportar una liberación de energía nuclear residual que domine las edades de enfriamiento de estas estrellas. Sin embargo, las edades obtenidas para púlsares de milisegundo a partir de su spin-down (bajo la hipótesis del modelo de dipolo magnético) parecen sugerir ${ }^{3}$ la necesidad de que muchas de las HeWD tengan envolturas de hidrógeno delgadas en el sentido de que la liberación residual de energía nuclear no resulte importante en el enfriamiento de las HeWDs. En esta tesis se muestra que la inclusión de la difusión de elementos en los cálculos evolutivos de las HeWDs, naturalmente conduce a una resolución de esta aparente paradoja. En efecto, las envolturas inicialmente gruesas de hidrógeno son reducidas durante episodios de flash termonucleares inducidos por la difusión que tienen lugar bien temprano en la evolución de estos objetos, que tienen posteriormente una evolución rápida a lo largo de su curva de enfriamiento. Se ha mostrado también que existe un rango de masas de las HeWDs en el que estos flashes tienen lugar. Fuera de este rango la evolución de las HeWDs está dominada por la combustión nuclear de su envoltura de hidrógeno.

Se mostró que un tratamiento detallado de la atmósfera de las HeWDs es necesario para obtener edades de enfriamiento precisas, ya que la profundidad que alcanza la envoltura convectiva a bajas $\mathrm{T}_{\text {eff }}$ en los modelos depende del tratamiento dado a la atmósfera.

A bajas $\mathrm{T}_{\text {eff }}$, la gran absorción en el infrarrojo inducida por colisiones (CIA) en la atmósfera de las HeWDs es una fuente de opacidades que determina el espectro emergente de estas estrellas. En particular mostramos que cuando la $\mathrm{T}_{\text {eff }}$ cae por debajo de unos $4000 \mathrm{~K}$, estas estrellas se vuelven azules a medida que se enfrían. Además la evolución por debajo de esta $T_{\text {eff }}$ ocurre a una magnitud visual aproximadamente constante. Este

\footnotetext{
${ }^{3}$ Es importante mencionar, sin embargo, que la validez del modelo dipolar para determinar las edades de los púlsares mediante su spin-down es cuestionada por diversos inverstigadores (ver p.ej., Camilo, Thorsett \& Kulkarni 1994).
} 
resultado, en combinación con las edades "cortas" de enfriamiento predichas por nuestros modelos que incluyen la difusión de elementos, muestra que muchas HeWDs frías pueden tener, al momento presente, colores "azules".

Estudiamos también la influencia de la metalicidad $Z$ de los progenitores en la formación y evolución de las HeWDs. Se consideraron modelos con $Z=0.02, Z=0.001$ y $Z=0.0002$. Encontramos que el rango de masas en que las HeWDs sufren flashes termonucleares depende de la metalicidad inicial de sus progenitores. Esto es importante a la hora de comparar modelos de enfriamiento con HeWDs observadas en medios donde la metalicidad sea distinta de la solar. En particular esto resulta importante con respecto a las HeWDs observadas recientemente en cúmulos globulares.

La segunda de las ramas mencionadas se refiere a las estrellas enanas blancas con núcleos de carbono-oxígeno (COWDs). En este caso el objetivo era bien distinto ya que se centraba en la obtención de modelos de COWDs detallados en cuanto a su estructura interna, particularmente en relación a su estratificación química. Estos modelos han sido utilizados para estudiar las propiedades pulsacionales de las estrellas DAV, también conocidas como estrellas ZZ Ceti (el estudio de las pulsaciones de estas estrellas, sin embargo, no entra dentro de los objetivos de esta tesis). En relación a estas estrellas, entonces, los resultados principales obtenidos son:

Se consideró la evolución desde la ZAMS hasta la etapa de ZZ Ceti de dos modelos estelares de 3 y $7.5 \mathrm{M}_{\odot}$. En particular se hizo hincapié en la evolución de estos progenitores de COWD durante la fase de los pulsos térmicos, en el extremo de la AGB, etapa en la cual la parte externa de los perfiles químicos iniciales de la WD remanente son formados. Se obtuvieron WDs remanentes de 0.563 y $0.936 \mathrm{M}_{\odot}$ respectivamente cuya evolución fue seguida hasta la etapa de ZZ Ceti.

Los perfiles químicos iniciales de los remanentes resultan profundamente modificados por los diferentes procesos de difusión. Los perfiles resultantes durante la etapa de ZZ Ceti no muestran las variaciones casi discontinuas que pueden observarse cuando la evolución como WD comienza. Por el contrario, los perfiles resultantes son suaves. Por otro lado, en ambos remanentes se observa una región en la cual la composición química tiene contribuciones apreciables de ${ }^{4} \mathrm{He},{ }^{12} \mathrm{C}$ y ${ }^{16} \mathrm{O}$. Además los perfiles evolucionan por efectos de la difusión aún durante la etapa de ZZ Ceti. Todo esto nos lleva a afirmar que el equilibrio difusivo no resulta una aproximación aceptable para determinar la forma de las interfaces químicas de las estrellas ZZ Ceti. Además, la aproximación de elemento-traza resulta completamente inadecuada ya que hay regiones donde dos y hasta tres especies químicas resultan abundantes. Ambas aproximaciones son usualemente empleadas en cálculos de pulsaciones de WDs.

En el caso del remanente de $0.563 \mathrm{M}_{\odot}$, la liberación de energía nuclear alcanza un máximo local al comienzo de la etapa de ZZ Ceti, contribuyendo con un $10 \%$ a la luminosidad superficial de la estrella. Por el contrario, en el caso del remanente más masivo, la liberación de energía nuclear es despreciable durante toda su evolución.

Los perfiles químicos resultantes, que no presentan variaciones abruptas, dan como resultado que el término de Ledoux tiene un perfil suave en el interior de la estrella, incluso en las interfaces. Esto resulta en una frecuencia de Brunt-Väisälä que tiene también un perfil suave. Estudios pulsacionales realizados con estos modelos muestran que esto tiene 
influencia sobre los llamados "modos atrapados". En efecto, nuestros modelos muestran que este efecto en mucho menos notorio que lo que generalmente se ha presentado en la literatura. Esto tiene importantes implicancias respecto a por qué no todos los modos esperados desde el punto de vista teórico son observados en las estrellas pulsantes. Finalmente, la distribución más suave de períodos que se obtiene al considerar consistentemente la difusión de elementos permite esperar que la señal producida en las pulsaciones por la cristalización en estrellas ZZ Ceti masivas sea detectable y no quede oculta por "ruido" en los períodos inducido por los perfiles químicos. 


\section{Apéndice: preguntas y comentarios del jurado}

\section{Sería conveniente explicitar el criterio utilizado para seleccionar las masas de los modelos calculados.}

La elección de las masas de los modelos calculados tiene por objetivo cubrir el rango de masas esperado para estas estrellas. Sin embargo cada valor particular (es decir, por qué $0.169 \mathrm{M}_{\odot}$ y no $\left.0.171 \mathrm{M}_{\odot}\right)$ resulta de la evolución previa de los mismos, es decir de la historia de pérdida de masa de los mismos, por lo que no son valores "elegidos" ad-hoc.

2. En la página 17 se menciona que el código calcula la tasa de emisión de neutrinos. Las pérdidas de energía por emisión de neutrinos pueden exceder las pérdidas por fotones en algunos casos. No sería conveniente incluir gráficos de $L_{\nu}$ vs Edad y $L_{\nu}$ vs $L$, para mostrar cómo evolucionan estas pérdidas? Cuál es la luminosidad media de una enana blanca de baja masa en neutrinos y cuál la de una de $\mathrm{CO}$ en las principales etapas de evolución?

Como primer punto, cabe mencionar que las pérdidas de energía por emisión de neutrinos se han incluido siguiendo a Itoh et al. (1996) y su tratamiento incluye los distintos sabores de neutrinos.

En el caso de la evolución de las enanas blancas de baja masa, con núcleos de helio, la emisividad de neutrinos juega un papel despreciable. Para ilustrar esto en la figura A.1 se muestra la evolución temporal de la luminosidad superficial y la correspondiente a la emisividad de neutrinos del modelo de HeWD de $0.449 \mathrm{M}_{\odot}$ durante las primeras fases de su evolución. La pérdida de energía por emisión de neutrinos es varios órdenes de magnitud más pequeña que la energía radiada por la estrella. Estas cantidades son comparables solamente en el intervalo $0<\log$ Age $<1$, es decir durante unos $10 \mathrm{Myr}$, por lo que la emisión de neutrinos resulta despreciable en lo concerniente a la determinación de las edades de enfriamiento de estos objetos. Se muestra el modelo de $0.449 \mathrm{M}_{\odot}$ ya que para masas menores la contribución de los neutrinos es aún menor.

En el caso de las enanas blancas con núcleos de carbono-oxígeno, se muestran en las figuras 6.6 y 7.3 de la tesis gráficos similares para los modelos de 0.563 y $0.936 \mathrm{M}_{\odot}$. En ambos casos la tasa de energía perdida por emisión de neutrinos excede a la radiada por la estrella en las primeras fases de la evolución de los modelos. Sin embargo la emisividad de neutrinos decae rápidamente cuando el núcleo se enfría, y en unos 100 Myr se vuelve un efecto secundario, prácticamente despreciable. Desde el punto de vista de las pulsaciones de las estrellas tipo ZZ Ceti, la emisión de neutrinos resulta completamente despreciable ya que cuando las estrellas alcanzan la banda de inestabilidad, la temperatura del núcleo de estas estrellas es demasiado baja como para que exista una emisión de neutrinos apreciable. 


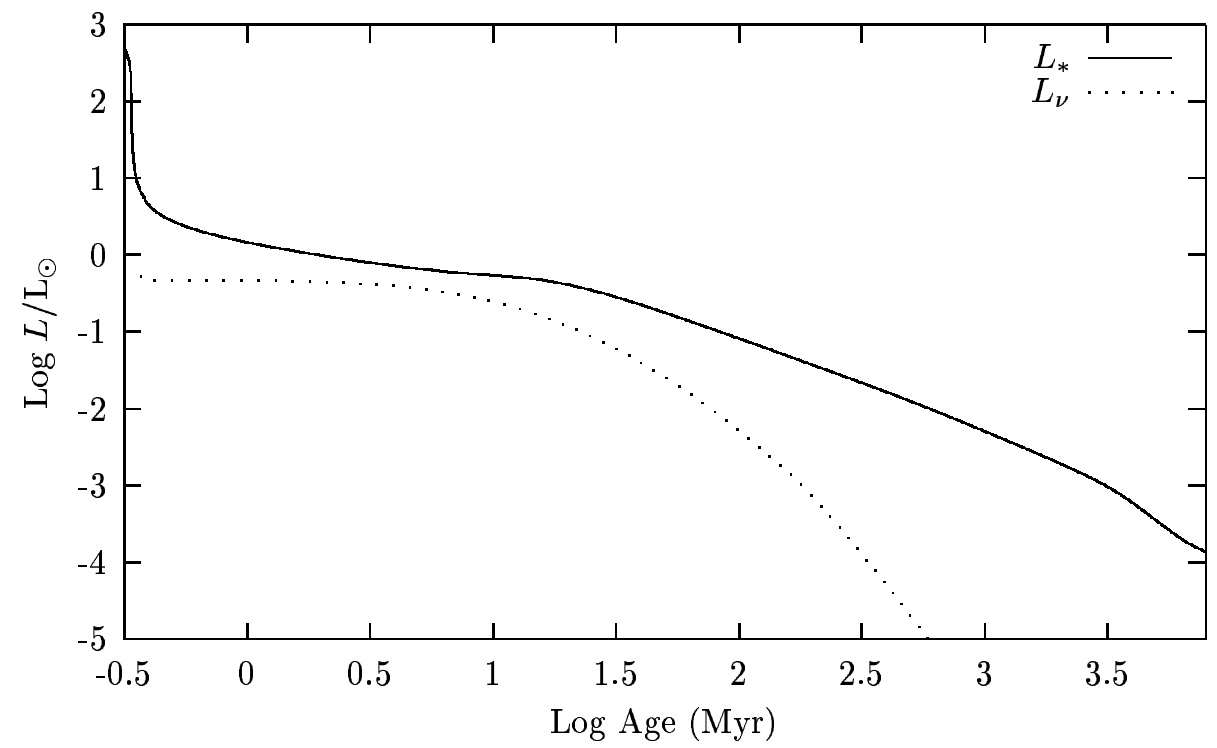

Figura A.1: Luminosidades superficial y por emisión de neutrinos en función de la edad para el modelo de HeWD de $0.449 \mathrm{M}_{\odot}$.

Vale la pena mencionar, sin embargo, que desde el punto de vista de las pulsaciones de enanas blancas, los neutrinos pueden resultar importantes en el caso de las enanas blancas DOV. Estas estrellas pulsantes son muy calientes, con temperaturas efectivas del orden de $10^{5} \mathrm{~K}$, y temperaturas centrales del orden de $60-80 \times 10^{6} \mathrm{~K}$, para las cuales la emisividad de neutrinos es un efecto dominante en la evolución de estos objetos. La evolución temporal de los períodos $(\dot{P})$ de los distintos modos de pulsación depende de la velocidad de enfriamiento de la enana blanca (que a su vez depende fuertemente de $L_{\nu}$ en estas etapas). Por este motivo, la emisión de neutrinos es importante en la determinación de $\dot{P}$ en las DOV. Invirtiendo el razonamiento, puede usarse la determinación observacional de $\dot{P}$ para obtener cotas para cantidades tales como, la tasa de emisividad de neutrinos, el momento dipolar magnético de los neutrinos.

3. Las estrellas progenitoras de las enanas blancas suelen tener campos magnéticos. Como el flujo magnético se conserva durante la fase de contracción, la intensidad del campo de la enana blanca resultante puede ser muy elevada. Schmidt \& Smith (1995), por ejemplo, dan una lista de 42 enanas blancas para las cuales se han medido campos con $B>10^{4} \mathrm{G}$. Por otro lado, medidas del efecto Zeeman muestran que hay enanas blancas con campos magnéticos de miles de MG. En el interior de estas estrellas, $B$ sería del orden de $10^{10}$ G. Es difícil pensar que esos campos no afecten los fenómenos de difusión, entre otras cosas. Podrías cuantificar de alguna manera la incidencia que podrían tener campos magnéticos fuertes y medios en los resultados que has obtenido? Cumple la presión magnética un rol importante? Puede incluirse este efecto en la teoría?

Las enanas blancas magnéticas tienen campos magnéticos entre $3 \times 10^{4}$ y $10^{9} \mathrm{G}$. Actualmente hay unas 70 estrellas de este tipo conocidas, lo que equivale aproximadamente a un $2 \%$ del total de las enanas blancas. De esta manera, la presencia de campos magnéticos de intensidad media o alta queda limitada a un grupo menor de enanas blancas. Es importante remarcar que el 
límite inferior para el campo magnético es un límite dado por la detectabilidad de estos campos.

Una cuantificación de la influencia de los campos magnéticos medios e intensos en la difusión de elementos está más allá del alcance de esta tesis. Creo, de hecho, que sería un tema en sí mismo para la realización de una tesis doctoral. En esta tesis se ha despreciado la presencia de campos magnéticos. A continuación se presenta una estimación aproximada de la intensidad a partir de la cual los campos magnéticos pueden tener una influencia apreciable en la difusión.

Supongamos que tenemos un medio formado por dos especies que denominamos 1 y 2 y que podemos identificar con protones y electrones. Debido a que la fuerza gravitatoria sobre los protones es más intensa que sobre los electrones, ésta tiende a separarlos, creando un campo eléctrico $E$ que contrarresta la separación. Supongamos que por unidad de volumen la carga neta es nula $\left(n_{e}=n_{p}\right)$, de esta manera el gradiente de concentración se anula y en equilibrio se tiene:

$$
m_{p} g+e E=m_{e} g-e E
$$

de donde

$$
E=-\frac{m_{p}+m_{e}}{2 e} g \approx-\frac{m_{p}}{2 e} g
$$

Puede verse que este resultado es consistente con la hipótesis de neutralidad de carga. En efecto,

$$
\vec{\nabla} \cdot \vec{E}=-4 \pi e\left(n_{p}-n_{e}\right)=-\frac{m_{p}}{2 e} \vec{\nabla} \cdot \vec{g}=\frac{m_{p}}{2 e} G 4 \pi n_{p} m_{p}
$$

de donde

$$
-\frac{n_{p}-n_{e}}{n_{p}}=\frac{G m_{p}^{2}}{2 e^{2}} \sim 4 \times 10^{-37}
$$

Consideremos ahora un elemento de volumen, la fuerza neta es

$$
F=n_{p}\left(m_{p} g+e E\right)+n_{e}\left(m_{e} g-e E\right)=n_{p} \frac{m_{p}+m_{e}}{2} g .
$$

En presencia de un campo magnético, las partículas estarán sujetas también a la fuerza de Lorentz

$$
F_{B} \leq \frac{1}{c} j B
$$

donde $j$ es la densidad de corriente. Dado que hay un flujo tanto de protones como de electrones, y además carga eléctrica nula, tendremos que

$$
j \leq n_{p} e v \rightarrow F_{B} \leq \frac{1}{c} n_{p} e v B
$$

donde $v$ es la velocidad de los iones. Entonces si $F_{B} \approx F$ tendremos

$$
\frac{1}{c} n_{p} e v B \approx n_{p} \frac{m_{p}+m_{e}}{2} g
$$

que lleva a

$$
B \approx \frac{m_{p}+m_{e}}{2} \frac{c g}{e v} \approx \frac{m_{p}}{2} \frac{c g}{e v}
$$


En el caso de una enana blanca, un valor típico de la aceleración de la gravedad es $g=10^{8} \mathrm{~cm} / \mathrm{s}^{2}$. La velocidad de la difusión depende fuertemente de la profundidad. Si $q=1-m / M_{*}$, para un valor $\log q=-10$ y una temperatura efectiva de unos $20000 \mathrm{~K}$, tenemos $v \approx 7 \times 10^{-6} \mathrm{~cm} / \mathrm{s}$. De esta manera

$$
B \approx 7 \times 10^{8} \text { gauss }
$$

De esta manera, puede verse que la influencia del campo magnético en la difusión resultará despreciable en el caso de las enanas blancas no magnéticas, que como se mencionó anteriormente representan aproximadamente al $98 \%$ de las enanas blancas conocidas. Desde ya que esta estimación no pretende ser del todo rigurosa. Una determinación correcta tanto de $v$ como de $j$ implica resolver las ecuaciones de difusión incluyendo consistentemente los efectos del campo magnético. Sin embargo, la estimación hecha de $B$ establece un límite seguro, al menos en cuanto al caso de las enanas blancas no magnéticas que tienen campos magnéticos, al menos, cuatro órdenes de magnitud menores. Además es interesante notar que desde el punto de vista de los resultados presentados en esta tesis, los efectos importantes de la difusión ocurren en regiones de la estrella correspondientes al rango $\log q \approx-2--6$ donde la velocidad de difusión es varios órdenes de magnitud menor que el valor usado más arriba para estimar $B$.

En relación a la influencia de la presión magnética en la estructura de la estrella, nos limitamos a los casos de enanas blancas no magnéticas, es decir a aquellos casos en que $B$ es a lo sumo del orden de $3 \times 10^{4}$ gauss. La presión magnética será del orden de

$$
P_{\text {mag }}=\frac{1}{8 \pi} B^{2} \approx 10^{9}-10^{10} \operatorname{din}_{\mathrm{cm}^{-2}}
$$

donde el valor mayor corresponde a regiones más internas de la estrella. Por otro lado, tomando por ejemplo un modelo de la secuencia de enana blanca de helio de $0.243 \mathrm{M}_{\odot}$, correspondiente a una $\mathrm{T}_{\mathrm{eff}}=20000 \mathrm{~K}$, la presión alcanza valores del orden de $10^{13}$ din $\mathrm{cm}^{-2}$ para $\log q \approx-8$ y crece hasta $10^{22-23}$ din $\mathrm{cm}^{-2}$ hacia el centro de la estrella. Estos valores muestran con claridad que la presión magnética no juega un papel importante en la determinación de la estructura de las enanas blancas no magnéticas. No puede descartarse, sin embargo, que los campos magnéticos sean importantes en las regiones más externas de la estrella (envoltura y atmósfera), en particular durante aquellas etapas en que se desarrolle una región convectiva.

4. Claramente dices en la página 8 que te limitas a situaciones con simetría esférica. Los efectos de la rotación son considerados despreciables en el análisis de la difusión. Las enanas blancas, sin embargo, rotan y se deforman. De nuevo, sería deseable algún tipo de análisis o comentario sobre como estos efectos pueden afectar los resultados obtenidos. Uno siempre parte de simplificaciones a la hora de modelar, pero siempre también es conveniente discutir esas simplificaciones y cuál es el grado de error que introducen o las razones que tenemos para pensar que esos errores pueden ser despreciables. Esto es particularmente importante cuando se hacen o proponen comparaciones directas con las observaciones.

Las enanas blancas son rotadores lentos. Los períodos característicos son al menos del orden de horas. Este hecho observacional resulta muy interesante desde el punto de vista de la evolución previa, que conduce a la formación de las enanas blancas. Tomemos por ejemplo el caso del Sol. Si la evolución del Sol llevara a la formación de una enana blanca sin pérdida de momento angular, la enana blanca resultante tendría un período de unos 2.5 minutos. Esto sugiere que gran parte del momento angular del progenitor de la enana blanca es perdido 
durante su evolución, durante las fases de pérdida de masa. Consideremos una enana blanca con un período de unos $20000 \mathrm{~s}$ y un radio de $10000 \mathrm{~km}$. Entonces, la aceleración centrífuga en la superficie es de unos $100 \mathrm{~cm} / \mathrm{s}^{2}$, que comparada a la aceleración de la gravedad de unos $10^{8} \mathrm{~cm} / \mathrm{s}$, muestra que desde el punto de vista de la estructura de la estrella, la rotación juega un papel despreciable.

Sin embargo, la rotación induce circulación de corrientes que pueden a su vez inducir procesos de mezcla. Tassoul \& Tassoul (1983, ApJ, 267, 334), encuentran que para una enana blanca de $0.8 \mathrm{M}_{\odot}$ con $L=10^{-2} \mathrm{~L}_{\odot}$ las escalas temporales para la mezcla por circulación de corrientes es mayor al tiempo de Hubble. Sin embargo, estos autores estimaron que la escala temporal para alcanzar una solución estacionaria es de unos $10^{10}$ años, mayor que la edad característica de enfriamiento. De esta manera no puede descartarse que la rotación tenga una influencia en las regiones superficiales de las enanas blancas, en particular de las muy calientes.

Es interesante mencionar que se conocen algunas enanas blancas con períodos de rotación del orden de minutos, pero estas enanas blancas presentan también campos magnéticos intensos, por lo que quedan fuera del alcance de los resultados presentados en esta tesis.

5. En la página 4 hablas de convección en la envoltura. Qué inestabilidades podrían producir convección en estas envolturas? Las ecuaciones 2.8-2.11 desprecian la convección en la ecuación de movimiento, pero lo incluyen en la ecuación de energía. Debe pensarse que estas ecuaciones provienen de una suerte de modelo de campo medio? O sea, con un promediado sobre escalas espaciales y temporales más cortas que las de interés?

La convección en las envolturas de las enanas blancas juega un papel muy importante en el enfriamiento de las mismas. Un ejemplo de esto, en el caso de las enanas blancas de baja masa se discute en el capítulo 5. En los casos relevantes en esta tesis la envoltura de las enanas blancas (de hidrógeno prácticamente puro) se vuelve inestable debido al aumento de la opacidad asociado con la ionización parcial del hidrógeno. Esto conduce a que el gradiente radiativo sea mayor que el gradiente adiabático, dando como resultado que el material resulta dinámicamente inestable.

En el problema global de la estructura estelar cantidades tales como presión, densidad, temperatura, etc. deben considerarse como funciones promediadas sobre superficies de radio constante. Sin embargo, estas cantidades no son exactamente constantes sobre estas superficies, sino que presentan ciertas fluctuaciones. Estas fluctuaciones, que tendrán una distribución estadística sobre la superficie, pueden crecer y dar lugar a movimientos macroscópicos locales no esféricos que tendrán también una distribución estadística sobre la superficie. En las ecuaciones básicas de la estructura estelar, la suposición de simetría esférica puede mantenerse interpretando las variables como promedios apropiados sobre estas superficies concéntricas.

Desde el punto de vista de la ecuación de energía, el movimiento macroscópico producido por la convección representa una contribución despreciable a la energía: las velocidades involucradas en los movimientos convectivos son del orden de $1-100 \mathrm{~m} \mathrm{~s}^{-1}$, mientras que la velocidad térmica de los núcleos y electrones es siempre varios órdenes de magnitud mayor. 


\section{Bibliografía}

Adams W. S., 1915, Publication of the Astronomical Society of the Pacific, 27, 236

Adams W. S., 1925, Proceedings of the National Academy of Sciences of USA, 11, 382. Erratum: Observatory, 49, 88

Alberts F., Savonije G. J., van den Heuvel E. P. J., Pols O. R., 1996, Nature, 380, 676

Alcock C., Illarionov A., 1980, The Astrophysical Journal, 235, 541

Alexander D. R., Ferguson J. W., 1994, The Astrophysical Journal, 437, 879

Althaus L. G., Benvenuto O. G., 1997, The Astrophysical Journal, 477, 313

Althaus L. G., Benvenuto O. G., 1998, Monthly Notices of the Royal Astronomical Society, 296, 206

Althaus L. G., Benvenuto O. G., 2000, Monthly Notices of the Royal Astronomical Society, $\mathbf{3 1 7}, 952$

Althaus L. G., Serenelli A. M., Benvenuto O. G., 2001a, Monthly Notices of the Royal Astronomical Society, 323, 471

Althaus L. G., Serenelli A. M., Benvenuto O. G., 2001a, Monthly Notices of the Royal Astronomical Society, 324, 617

Althaus L. G., Serenelli A. M., Benvenuto O. G., 2001a, The Astrophysical Journal, 554, 1110

Althaus L. G., Serenelli A. M., Córsico A. H., Benvenuto O. G., 2002a, Monthly Notices of the Royal Astronomical Society, 330, 685

Anders E., Grevesse N., 1989, Geochim. Cosmochim. Acta, 53, 197

Anderson S. F., Margon B., Deutsch E. W., Downes R. A., Allen R. G., 1997, The Astrophysical Journal, 482, L69

Angulo C., et al., 1999, Nucl. Phys. A, 656, 3

Anthony-Twarog B. J., Twarog B. A., 2000, The Astronomical Journal, 120, 3111

Arnett D., Truran J., 1969, The Astrophysical Journal, 157, 1369

Arnett D., 1996, Supernovae and Nuclesynthesis, Princeton Universuty Press, Princeton

Benvenuto O. G., Althaus L. G., 1998, Monthly Notices of the Royal Astronomical Society, 293, 177

Bergeron P., Leggett S. K., Ruiz M. T., 2001, The Astrophysical Journal Supplement Series, 133, 413

Bergeron P., Ruiz M. T., Leggett S. K., 1997, The Astrophysical Journal Supplement Series, 
108, 339

Bergeron P., Saffer R. A., Liebert J., 1992, The Astrophysical Journal, 394, 228

Bergeron P., Saumon D., Wesemael F., 1995, The Astrophysical Journal, 443, 764

Bergeron P., Wesemael F., Beauchamp A., 1995, Publication of the Astronomical Society of the Pacific, 107, 1047

Bergeron P., Wesemael F., Fontaine G., 1992, The Astrophysical Journal, 387, 288

Bessell M. S., 1990, Publication of the Astronomical Society of the Pacific, 102, 1181

Bessell M. S., Brett J. M., 1988, Publication of the Astronomical Society of the Pacific, 100, 1134

Blöcker T., 1995a, Astronomy \& Astrophysics, 297, 727

Blöcker T., 1995b, Astronomy \&5 Astrophysics, 299, 755

Borysow A., Jorgensen U. G., Fu Y., 2001, JQSRT, 68, 235

Borysow A., Jorgensen U. G., Zheng C., 1997, Astronomy \& Astrophysics, 324, 185

Bradley P. A., 1996, The Astrophysical Journal, 468, 350

Bradley P. A., 1998, The Astrophysical Journal Supplement Series, 116, 307

Bradley P. A., 2001, The Astrophysical Journal, 552, 326

Bradley P. A., Winget D. E., 1994, The Astrophysical Journal, 430, 850

Bragaglia A., Renzini A., Bergeron P., 1995, The Astrophysical Journal, 443, 735

Brassard P., Fontaine G., Wesemael F., Kawaler S. D., Tassoul M., 1991, The Astrophysical Journal, 367, 601

Brassard P., Fontaine G., Wesemael F., Hansen C. J., 1992a, The Astrophysical Journal Supplement Series, 80, 369

Brassard P., Fontaine G., Wesemael F., Tassoul M., 1992b, The Astrophysical Journal Supplement Series, 81, 747

Brickhill A. J., 1991, Monthly Notices of the Royal Astronomical Society, 251, 673

Brown T.M., Gilliland R. L., 1994, Annual Review of Astronomy 8 Astrophysics, 32, 37

Burderi L., D’Antona F. Burgay M., 2002, The Astrophysical Journal, 574, 325

Burgers J.M., 1969, Flow Equations for Composite Gases, Academic, New York

Callanan P. J., Garnavich P. M., Koester D., 1998, Monthly Notices of the Royal Astronomical Society, 298, 211

Camilo F., Thorsett, Kulkarni S. R., 1994, The Astrophysical Journal, 412, 215

Castellani M., Castellani V., 1993, The Astrophysical Journal, 407, 649

Caughlan G. R., Fowler W. A., 1988, Atomic Data and Nuclear Data Tables, 36, 411

Caughlan G. R., Fowler W. A., Harris M. J., Zimmermann B. A., 1985, Atomic Data and Nuclear Data Tables, 32, 197

Chandrasekhar S., 1931a, Phil. Magazine, 11, 592 
Chandrasekhar S., 1931b, The Astrophysical Journal, 74, 81

Chandrasekhar S., 1939, An Introduction to the Study of Stellar Structure, Cambridge University Press, Cambridge

Chin C. W., Chiu H. Y., Stothers R., 1966, Ann. Phys., 39, 280

Clayton D. D., 1968, Principles of Stellar Evolution and Nucleosynthesis, The University of Chicago Press, Chicago

Cool A. M., Grindlay J. E., Cohn H. N.,Lugger P. M., Bailyn C. D., 1998, The Astrophysical Journal, 508, L75

Córsico A. H., 2002, Tesis doctoral, Facultad de Ciencias Astronómicas y Geofísicas, Universidad Nacional de La Plata, La Plata

Córsico A. H., Althaus L. G., Benvenuto O. G., Serenelli A. M., 2001, Astronomy E6 Astrophysics, 380, L17

Córsico A. H., Althaus L. G., Benvenuto O. G., Serenelli A. M., 2001, Astronomy EG Astrophysics, 387, 531

Córsico A. H., Benvenuto O. G, 2002, Astrophysics and Space Science, 279, 281

Cox J. P., 1980, Theory of Stellar Pulsations, Princeton University Press, Princeton, NJ

D’Antona F., Mazzitelli I., 1989, The Astrophysical Journal, 347, 934

D’Antona F., Mazzitelli I., 1990, Annual Review of Astronomy \& Astrophysics, 28, 139

Danziger I. J., Baade D., Della Valle M., 1993, Astronomy \& Astrophysics, 276, 382

Däppen W., Anderson L., Mihalas D., 1987, The Astrophysical Journal, 319, 195

Dolez N., Vauclair G., 1981, Astronomy \& Astrophysics, 102, 375

Domínguez I., Chieffi A., Lamongi M., Straniero O., 1999, The Astrophysical Journal, 524, 226

Driebe T., Blöcker T., Schönberner D., Herwig F., 1999, Astronomy \&6 Astrophysics, 350, 89

Driebe T., Schönberner D., Blöcker T., Herwig F., 1998, Astronomy 66 Astrophysics, 339, 123

Dupuis J., Fontaine G., Pelletier C., Wesemael F., 1992, The Astrophysical Journal Supplement Series, 82, 505

Edmonds P. D., Gilliland R. L., Heinke C. O., Grindlay J. E., Camilo F., 2001, The Astrophysical Journal, 557, L57

Edmonds P. D., Grindlay J., Cool A., Cohn H., Lugger P., Bailyn C., 1999, The Astrophysical Journal, 516, 250

Eggleton P. P., 1983, The Astrophysical Journal, 268, 368

Fontaine G., Brassard P., Bergeron P., 2001, Publication of the Astronomical Society of the Pacific, 113, 409

Fontaine G., Michaud G., 1979, The Astrophysical Journal, 231, 826

Gautschy A., Ludwig H, Freytag B., 1996, Astronomy \& Astrophysics, 311, 493

Gautschy A., Saio H., 1995, Annual Review of Astronomy $\&$ Astrophysics, 33, 75 
Gautschy A., Saio H., 1996, Annual Review of Astronomy \& Astrophysics, 34, 551

Goldreich P., Wu Y., 1999, The Astrophysical Journal, 511, 904

Grossman S. A., Narayan R., Arnett D., 1993, The Astrophysical Journal, 407, 284

Grossman S. A., Taam R. E., 1996, Monthly Notices of the Royal Astronomical Society, 283, 1165

Gustafsson M., Frommhold L., 2001, The Astrophysical Journal, 546, 1168

Fowler R. H., 1926, Monthly Notices of the Royal Astronomical Society, 87, 114

Hansen B. M. S., 1998, Nature, 394, 860

Hansen B. M. S., 1999, The Astrophysical Journal, 520, 680

Hansen B. M. S., Kawaler S. D., 1989, Stellar interiors, Springer, New York

Hansen B. M. S., Phinney E. S., 1998, Monthly Notices of the Royal Astronomical Society, 294, 557

Hansen B. M. S., Phinney E. S., 1998b, Monthly Notices of the Royal Astronomical Society, 294, 569

Harris H. C., Dahn C. C., Vrba F. J., Henden A. A., Liebert J., Schmidt G. D., Reid I. N., 1999, The Astrophysical Journal, 524, 1000

Hine B. P., Nather R. E., 1987 en Philip A. G. D., Hayes D. S., Liebert J., eds, Proc. IAU Colloq. 95. The second COnf. on Faint BLue Stars. L. Davis Press, p. 627

Holtzman J. A., Burrows C. J., Casertano S., Hester J. J., Trauger J. T., Watson A. M., Worthey G., 1995, Publication of the Astronomical Society of the Pacific, 107, 1065

Hubbard W. B., Lampe M., 1969, The Astrophysical Journal Supplement Series, 18, 297

Hummer D. G., Mihalas D., 1988, The Astrophysical Journal, 331, 794

Iben I. Jr., 1982, The Astrophysical Journal, 260, 821

Iben I. Jr., 1984, The Astrophysical Journal, 277, 354

Iben I. Jr., Livio M., 1993, Publication of the Astronomical Society of the Pacific, 105, 1373

Iben I. Jr., MacDonald J., 1985, The Astrophysical Journal, 296, 540

Iben I. Jr., MacDonald J., 1986, The Astrophysical Journal, 301, 164

Iben I. Jr., Renzini A., 1983, Annual Review of Astronomy \& Astrophysics, 21, 271

Iben I. Jr., Tutukov A. V., 1984, The Astrophysical Journal, 282, 615

Iben I. Jr., Tutukov A. V., 1986, The Astrophysical Journal, 311, 742

Iben I. Jr., Tutukov A. V., Yungelson L., 1997, The Astrophysical Journal, 475, 291

Iglesias C. A., Rogers F. J., 1996, The Astrophysical Journal, 464, 943

Irwin A. W., 1981, The Astrophysical Journal Supplement Series, 45, 261

Itoh N., Hayashi H., Nishikawa, A., Kohyama Y., 1996, The Astrophysical Journal Supplement Series, 102, 411

Itoh N., Mitake S., Iyetomi H., Ichimaru S., 1983, The Astrophysical Journal, 273, 774 
Jorgensen U. G., Hammer D., Borysow A., Falkesgaard J., 2000, Astronomy \& Astrophysics, $\mathbf{3 6 1}, 283$

Kanaan a., Kepler S. O., Giovannini O., Díaz M., 1992, The Astrophysical Journal, 390, L89

Kaplan S. A.1949Naukovy Zapiski15109

Kaspi V. M., Taylor J. H., Ryba M. F., 1994, The Astrophysical Journal, 428, 713

Kawaler S. D., 1988, The Astrophysical Journal, 334, 220

Kawaler S. D., Winget D. E., Hanses C. J., Iben I. Jr., 1986, The Astrophysical Journal, 306, L41

Kippenhahn R., Kohl K., Weigert A., 1967, Z. Astrophys, 66, 58

Kippenhahn R., Thomas H.-C., Weigert A., 1968, Z. Astrophys, 69, 265

Kippenhahn R. \& Weigert A., 1990, Stellar Structure and Evolution, Springer Verlag, Berlin

Kippenhahn R., Weigert A., Hofmeister E., 1967, Methods of Computational Physics 7, Interscience, New York

Koester D., Schönberner D., 1986, Astronomy \& Astrophysics, 154, 125

Kovetz A., Shaviv G., 1970, Astronomy \& Astrophysics, 8, 398

Kurucz R. L., 1979, The Astrophysical Journal Supplement Series, 40, 1

Lamb D. Q., Van Horn H. M., 1975, The Astrophysical Journal, 200, 306

Landau L. D., Lifshitz E. M., 1971, Classical theory of fields, Pergamon Press, Oxford

Landsman W., Aparicio J., Bergeron P., Di Stefano R., Stecher T.P., 1997, The Astrophysical Journal, 481, L93

Lorimer D. R., Festin L., Lyne A. G., Nicastro L., 1995, Nature, 376, 393

Lundgren S. C., Cordes J. M., Foster R. S., Wolszczan A., Camilo F., 1996, The Astrophysical Journal, 458, L33

Magni G., Mazzitelli I., 1979, Astronomy \& Astrophysics, 72, 134

Marsh T. R., 1995, Monthly Notices of the Royal Astronomical Society, 275, L1

Marsh T. R., Dhillon V. S. \& Duck S. R., 1995, Monthly Notices of the Royal Astronomical Society, 275, 828

Maxted P. F. L., Marsh T. R., Moran C. H. J., Zan Z., 2000, Monthly Notices of the Royal Astronomical Society, 314, 334

Mazzitelli I., D'Antona F., 1986, The Astrophysical Journal, 308, 706

Mestel L., 1952, Monthly Notices of the Royal Astronomical Society, 112, 583

Mestel L., Ruderman M. A., 1967, Monthly Notices of the Royal Astronomical Society, 136, 27

Montgomery M. H., Metcalfe T. S., Winget D. E., 2001, The Astrophysical Journal, 548, L53

Montgomery M. H., Winget D. E., 1999, The Astrophysical Journal, 526, 976

Moran C., Marsh T. R., Bragaglia A., 1997, Monthly Notices of the Royal Astronomical Society, 
288, 538

Muchmore D., 1984, The Astrophysical Journal, 278, 769

Neale L., Tennyson J., 1995, The Astrophysical Journal, 454, L169

Orosz J. A., Wade R. A., Harlow J. J. B., Thorstensen J. R., Taylor C. J., Eracleous M., 1999, The Astronomical Journal, 117, 1598

Ostlie D., Cox A. N., 1986, The Astrophysical Journal, 311, 864

Paquette C., Pelletier C., Fontaine G., Michaud G., 1986a, The Astrophysical Journal Supplement Series, 61, 177

Paquette C., Pelletier C., Fontaine G., Michaud G., 1986b, The Astrophysical Journal Supplement Series, 61, 197

Podsiadlowski Ph., Joss P. C., Hsu J. J. L., 1992, The Astrophysical Journal, 391, 246

Press W. H., Teukolsky S. A., Vetterling W. T., Flannery B. P., 1986, Numerical Recipes, CAmbridge University Press, Cambridge

Richer H. B., Falhman G. G., Rosvick J., Ibata R. A., 1998, The Astrophysical Journal, 504, L91

Richer H. B. et al., 1997, The Astrophysical Journal, 484, 741

Ritter H., 1988, Astronomy \& Astrophysics, 202, 93

Rohrmann R. D., 2001, Monthly Notices of the Royal Astronomical Society, 323, 699

Rohrmann R. D., Serenelli A. M., Althaus L. G., Benvenuto O. G., 2002, Monthly Notices of the Royal Astronomical Society, 335, 499

Saffer R. A., Livio M., Yungelson L. R., 1998, The Astrophysical Journal, 504, 392

Salaris M., Domínguez I., García-Berro E., Hernanz M., Isern J., Mochkovitch R., 1997, The Astrophysical Journal, 486, 413

Salaris M., García-Berro E., Hernanz M., Isern J., Saumon D., 2000, The Astrophysical Journal, $\mathbf{5 4 4}, 1036$

Sarna M. J., Ergma E., Antipova J., 2000, Monthly Notices of the Royal Astronomical Society, 316, 84

Saumon D., Bergeron P., Lunine J. I, Hubbard W. B., Burrows A., 1994, The Astrophysical Journal, 424, 333

Saumon D., Jacobson S. B., 1999, The Astrophysical Journal, 511, L107

Sauval A. J., Tatum J. B., 1984, The Astrophysical Journal Supplement Series, 56, 193

Savedoff M. P., Van Horn H. M., Vila S. C., 1969, The Astrophysical Journal, 155, 221

Schatzman E., 1956, Astron. Zhur., 33, 800

Schatzman E., 1958, White Dwarfs, North-Holland, Amsterdam

Schönberner D., Driebe T., Blöcker T., 2000, Astronomy \& Astrophysics, 356, 929

Schönberner D., 1979, Astronomy \& Astrophysics, 79, 108

Serenelli A. M., Althaus L. G., Rohrmann R. D., Benvenuto O. G., 2001, Monthly Notices of 
the Royal Astronomical Society, 325, 607

Serenelli A. M., Althaus L. G., Rohrmann R. D., Benvenuto O. G., 2002, Monthly Notices of the Royal Astronomical Society, en prensa, astro-ph/0208408

Tauris T. M., Savonije G. J., 1999, Astronomy \& Astrophysics, 350, 928

Tassoul M., Fontaine G., Winget D. E., 1990, The Astrophysical Journal Supplement Series, $\mathbf{7 2}, 335$

Taylor J. M., Grindlay J. E., Edmonds P. D., Cool A. M., 2001, The Astrophysical Journal, 553, L169

Thielemann F. K., Nomoto K., Hashimoto M., 1996, The Astrophysical Journal, 460, 408

Townsley D. M. \& Bildsten L., 2002, The Astrophysical Journal, 565, L35

Unglaub K., Bues I., 2000, Astronomy \& Astrophysics, 359, 1042

Unno W., Osaki Y., Ando H., Saio H., Shibahashi H., 1989, Nonradial Oscillations of Stars, 2nd edn., Tokio University Press, Tokyo

Van Horn H. M., 1968, The Astrophysical Journal, 151, 227

van Kerkwijk M. H., Bell J. F., Kaspi V. M., Kulkarni S. R., 2000, The Astrophysical Journal, 530, L37

van Kerkwijk M. H., Bergeron. P, Kulkarni S. R., 1996, The Astrophysical Journal, 467, L89

van Straten W., Bailes M., Britton M., Kulkarni S. R., Anderson S. B., Manchester R. N., Sarkissian J., 2001, Nature, 412, 158

Vassiliadis E. \& Wood P. R., 1993, The Astrophysical Journal, 413, 641

von Hippel T., 1998, The Astronomical Journal, 115, 1536

von Hippel T., Gilmore G., 2000, The Astronomical Journal, 120, 1384

Webbink R. F., 1975, Monthly Notices of the Royal Astronomical Society, 171, 555

Winget D. E., Kepler S. O., Kanaan A., Montgomery M. H., Giovannini O., 1997, The Astrophysical Journal, 487, L191

Winget D. E., Van Horn H. M., Tassoul M., Hansen C. J., Fontaine G., Carroll B. W., 1982, The Astrophysical Journal, 252, L65

Winget D. E., 1988, in Christensen-Dalsgaard E. J., Frnadsen S., eds, Proc. IAU Symp. 123, Advances in Helio- and Asteroseismology, Reidel, Dordrecht, p.305

Wood P. R., Faulkner D. J., 1986, The Astrophysical Journal, 307, 659

Zoccali M., 2001, The Astrophysical Journal, 553, 733 